\title{
Plasticity-dependent modulation of mitochondrial biogenesis determining motor neuron function and vulnerability
}

\author{
Dissertation \\ for the award of the degree \\ "Doctor of Philosophy" \\ Division of Mathematics and Natural Sciences \\ within the doctoral program Molecular Physiology of the Brain \\ of the Georg-August University School of Science (GAUSS)
}

submitted by

Camille Lancelin

from Paris, France

Göttingen 2015 


\section{Members of the Thesis Committee}

Dr. Till Marquardt, Supervisor

Developmental neurobiology

European Neuroscience Institute, Göttingen

Pr. Nils Brose, reviewer

Dept. of Molecular Neurobiology

Max Planck Institute for Experimental Medicine, Göttingen

Dr. Judith Stegmüller

Dept. of Cellular \& Molecular Neurobiology

Max Planck Institute for Experimental Medicine, Göttingen

\section{Members of the Examination Board}

Referee: Camin Dean, PhD

Trans-synaptic Signaling

European Neuroscience Institute, Göttingen

$2^{\text {nd }}$ Referee: Prof. Dr. Michael Hörner

Dept. of Cellular Neurobiology

Johann-Friedrich-Blumenbach-Institute for Zoology and Anthropology, Göttingen

$3^{\text {rd }}$ referee: Prof. Dr. Ralf Heinrich

Dept. Cellular Neurobiology

Schwann-Schleiden Research Centre, Göttingen

Date of oral examination: $29^{\text {th }}$ September, 2015 


\section{Affidavit}

I hereby, declare that this $\mathrm{PhD}$ thesis "Plasticity-dependent modulation of mitochondrial biogenesis determining motor neuron function and vulnerability" has been written independently with no other aids or sources than quoted.

Camille Lancelin

September, 2015

Göttingen, Germany 


\section{Table of content}

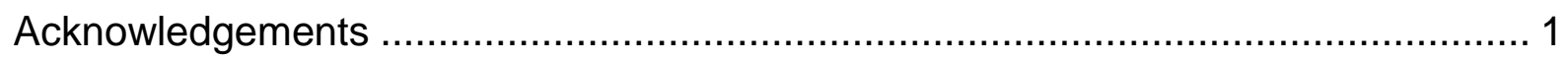

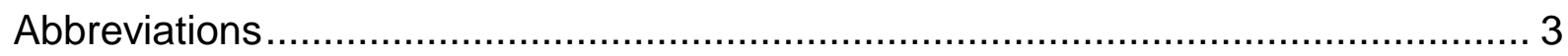

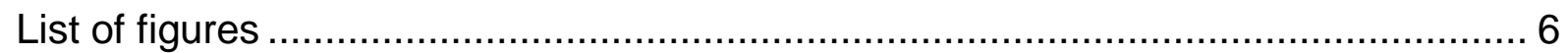

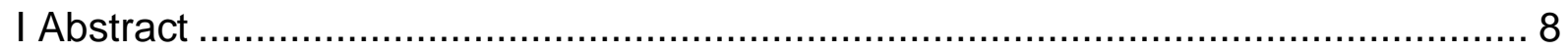

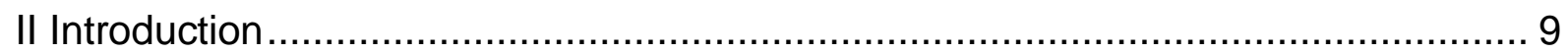

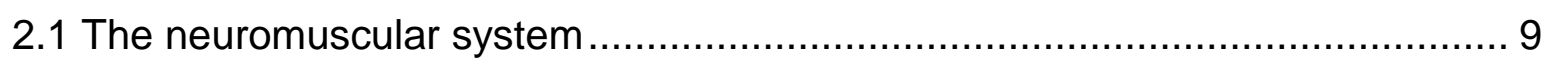

2.1.1 Spinal motor neurons and motor neuron diversity ................................ 10

2.1.2 Skeletal muscles and skeletal muscle diversity ..................................... 12

2.1.3 Organization of the motor system and types of motor units.................... 15

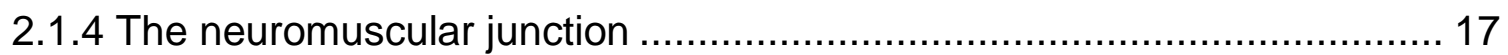

2.2 Neuromuscular adaptations to exercise .................................................. 19

2.2.1 Whole-body adaptations to exercise .................................................. 19

2.2.2 Skeletal muscle adaptations to exercise............................................... 21

2.2.3 Modifications in spinal motor neurons upon physical training ................... 24

2.2.4 Changes in the neuromuscular junction induced by exercise .................. 26

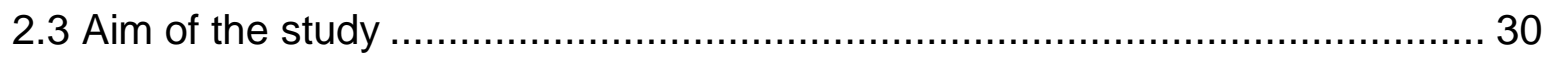

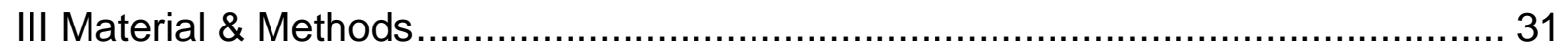

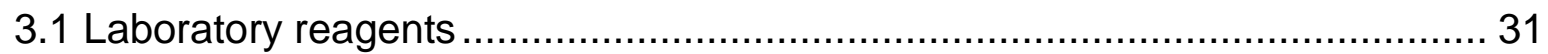

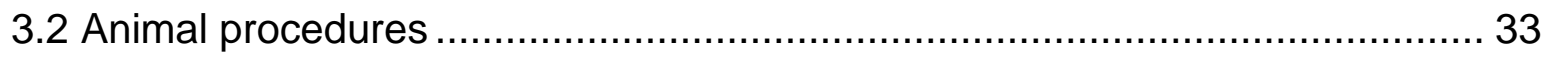

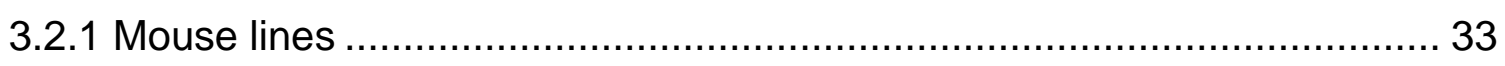

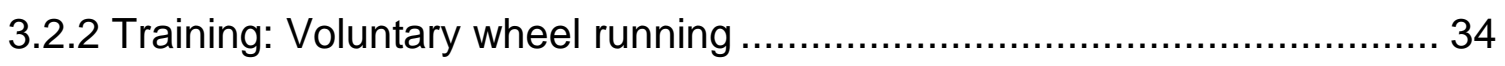

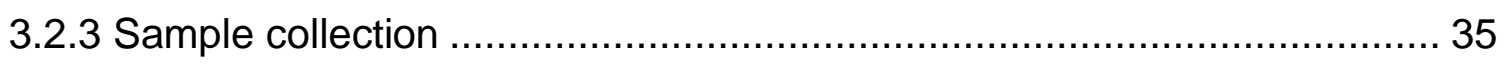

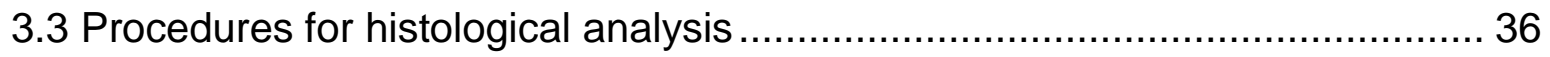

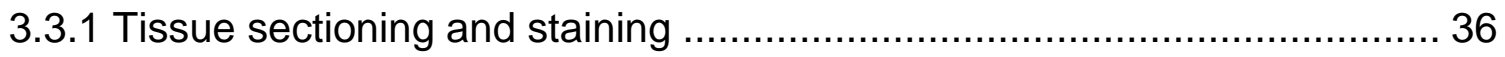

3.3.2 Imaging, 3-D reconstruction and statistical analysis ............................... 37

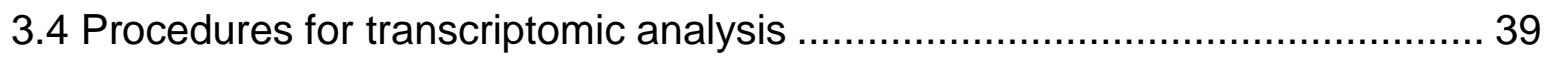

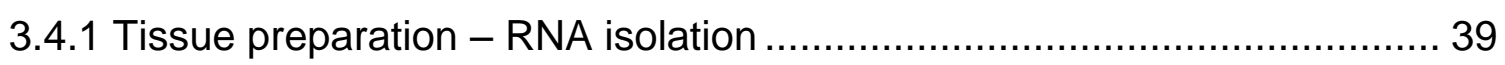

3.4.2 RNA sequencing and data analysis ................................................ 40 
IV Results

4.1 Endurance training in wild type mice

4.2 Chronic training induced a shift towards a slower type in slow and fastintermediate muscles 43

4.3 Remodeling of NMJ mitochondrial network by exercise ............................ 45

4.3.1 Characterization of NMJs from slow and fast muscles ......................... 46

4.3.2 Exercise-induced adaptations of mitochondrial network in NMJs.

4.4 Transcriptional changes in muscle and MNs following long-term voluntary wheel running.

4.4.1 Assessing sample quality and reliability ....................................... 52

4.4.2 Common transcriptional changes in muscle and spinal cord ................... 53

4.4.3 Transcriptional changes in the soleus ......................................... 56

4.4.4 Transcriptional changes specific to the neuromuscular synapse............. 57

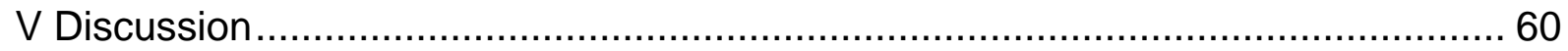

5.1 Physical training - exercise paradigm ............................................. 60

5.2 Exercise-induced specific adaptations in muscles ................................. 61

5.3 Neuromuscular system adaptation to training, the common changes .............6 62

5.4 Changes at the NMJ - synapse assembly and mitochondrial network .......... 66

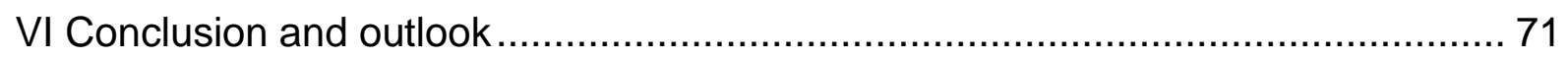

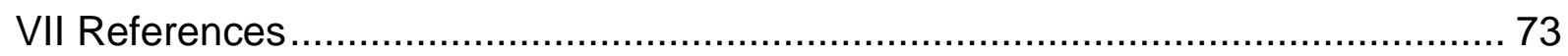

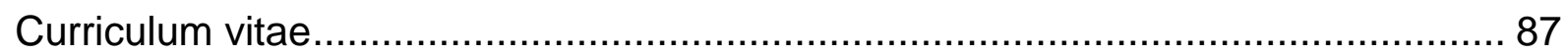




\section{Acknowledgements}

First of all, I would like to thank my thesis supervisor Dr. Till Marquardt for giving me the opportunity to work in the stimulating environment of his lab. There is no doubt that this "long-term endurance training" experience has durably changed my outlook on life.

I wish to express my sincere thanks to my thesis committee members Pr. Nils Brose and Dr. Judith Stegmüller for sharing their valuable comments and suggestions on the occasion of my thesis committees.

I also want to thank both Judith and Till for giving me the chance to develop an interesting and fruitful collaboration. In this regard, I would like to acknowledge the friendly working atmosphere that Siv Vingill and Chaitali Mukherjee created during our collaboration.

I thank all the members of the Marquardt lab, past and present, for sharing the lab with me: Dr. Pitchaiah Cherukuri, Dr. Lukas Cyganek, Dr. Piotr Fabrowski, Mudassar Khan, Pierre Klein, Alexandra Klusowski, Dr. Jacqueline Kueh, Dr. Tsung-I Lee (Veltine), Dr. Daniel Müller, Dr. Chor Hoon Poh (Anne), Nidhi Subhashini, Dr. Liang Wang, and Beate Weith, as well as our great students Yehan Bian, Luisa Heyer and Eric Schoger. Particularly, I would like to express my enormous gratitude to Alex and Pitchaiah whom support has been crucial along the four years of my PhD. Thanks Alex for the your moral support in difficult times and your logistic support at any time. Thanks Pitchaiah for your enthusiasm, your wisdom, your endless support. Life in the lab is not the same when you're not around. Many thanks also to Jacque for your friendship and the nice chats "around the cryostat". And a big thank to Beate for all the genotyping and to Pierre for excellent technical support.

I also want to acknowledge my collaborators Pr. Stefan Jakobs and Lena Grosse for their advice regarding mitochondria in general and their work on the STED analysis. Many thanks to the members of Dr. Stefan Bonn's and Pr. André Fischer's labs for the nice atmosphere they contributed to spread all over ENI's third floor. Special thanks go to Susanne Burkhardt, Anna-Lena Schütz, Vincenzo Capece and Dr. Cemil Kerimoglu for their great help on the RNAseq experiment. And thanks very 
much to Dr. Tonatiuh Pena Centeno for our interesting and stimulating discussions on statistical methods and strategies.

Sincere thanks also to our microscopy specialists Dr. Nils Halbsgut and Dr. Peter Wehner for their help and advice.

Special thank to Dr. Nicolas Stifani and Dr. Katia Burk for their proofreading, corrections and comments on the manuscript introduction.

I thank all my friends around the world, in Germany, in France, in Canada, or in Singapoure for keeping me in touch with the world, the one outside the lab. Particular thanks go to Elsa, Julien, Séverine, Lydia, Johanne and Vincent for not forgetting me. Finally, I would like to thank my family, my parents, my brothers Nicolas and Benoît (the R magician), and my wonderful boyfriend Pierre. I owe you so much that I can't find the words to express my gratitude. Je suis tellement chanceuse de vous avoir. Merci d'être là, toujours, et de croire en moi. 


\section{Abbreviations}

\section{Genes \& proteins}

\begin{tabular}{|c|c|}
\hline$\alpha B t x$ & Bungarotoxin subunit $A$ \\
\hline AChR (Chrn) & Acetylcholine receptor \\
\hline AChE (Ache) & Acetylcholinesterase \\
\hline ABI2 (Abi2) & Abl-interactor 2 \\
\hline AKT (Akt1/2) & Thymoma viral proto-oncogene $1 / 2$ / protein kinase $B$ \\
\hline AP2B (Tfap2b) & Transcription factor AP-2 beta \\
\hline ATPase & Adenosine tri-phosphatase \\
\hline BCKDH E1-a (Bckdha) & Branched chain ketoacid dehydrogenase E1 alpha, mitochondrial \\
\hline BDNF (Bdnf) & Brain-derived neurotrophic factor \\
\hline CACB4 (Cacnb4) & Calcium channel, voltage-dependent, beta 4 subunit \\
\hline CGRP-1 (Calca) & Calcitonin gene-related peptide 1 / polypeptide alpha \\
\hline ChAT (Chat) & Choline acetyltransferase \\
\hline Chondrolectin (Chod) & Chondrolectin \\
\hline DLK1 (DIk1) & Delta-like homolog 1 (Drosophila) \\
\hline DNase & Deoxyribonuclease \\
\hline DNM1L (Dnm1) & Dynamin 1-like / DRP1 \\
\hline Dystrophin (Dmd) & Dystrophin, muscular dystrophy \\
\hline ERR1 (Esrra) & Estrogen-related receptor alpha \\
\hline ERR2 (Esrrb) & Estrogen-related receptor beta \\
\hline ETHE1 (Ethe1) & Ethylmalonic encephalopathy 1 , mitochondrial \\
\hline FMR1 $(F m r 1)$ & Fragile $X$ mental retardation syndrome 1 \\
\hline GDNF (Gdnf) & Glial cell line-derived neurotrophic factor \\
\hline GLUT4 (SIc2a4) & Solute carrier family 2 (facilitated glucose transporter), member 4 \\
\hline HDAC5 (Hdac5) & Histone deacetylase 5 \\
\hline HOMER1/2 (Homer1/2) & Homer scaffolding protein $1 \& 2$ / Homer protein homolog $1 \& 2$ \\
\hline IGF-1 (lgf1) & Insulin-like growth factor 1 \\
\hline ISL-2 ( $|s| 2)$ & Insulin related protein 2 (islet 2) \\
\hline KCIP2 (Kcnip2) & Kv channel-interacting protein 2 \\
\hline KDM5A (Kdm5a) & Lysine (K)-specific demethylase $5 \mathrm{~A}$ \\
\hline KIFC3 (Kifc3) & Kinesin family member C3 \\
\hline LBX1 (Lbx1) & Ladybird homeobox homolog 1 (Drosophila) \\
\hline LHX3 (Lhx3) & LIM homeobox protein 3 \\
\hline LMX1B $(L m \times 1 b)$ & LIM homeobox transcription factor 1 beta \\
\hline MEF2 (Mef2) & Myocyte enhancer factor 2 \\
\hline Mitofusin-2 (Mfn2) & Mitofusin-2 \\
\hline
\end{tabular}




\begin{tabular}{|c|c|}
\hline MLYCD (Mlycd) & Malonyl-CoA decarboxylase, mitochondrial \\
\hline MMP9 (Mmp9) & Matrix metalloproteinase 9 \\
\hline MNX1 (MnX1) & Motor neuron and pancreas homeobox 1 / HB9 \\
\hline mTOR (Mtor) & Mammalian/Mechanistic target of rapamycin \\
\hline MUL1 (Mul1) & Mitochondrial ubiquitin ligase activator of NFKB 1 \\
\hline $\begin{array}{l}\text { MyHC I / Myosin-7 } \\
(\text { Myh7) }\end{array}$ & $\begin{array}{l}\text { Myosin Heavy Chain slow isoform / Myosin, heavy polypeptide } 7 \text {, cardiac } \\
\text { muscle, beta }\end{array}$ \\
\hline $\begin{array}{l}\text { MyHC Ila / Myosin-2 } \\
\text { (Myh2) }\end{array}$ & $\begin{array}{l}\text { Myosin Heavy Chain Ila / Myosin, heavy polypeptide 2, skeletal muscle, } \\
\text { adult }\end{array}$ \\
\hline $\begin{array}{l}\text { MyHC Ilb / Myosin-4 } \\
(\text { Myh4) }\end{array}$ & Myosin Heavy Chain Ilb / Myosin, heavy polypeptide 4, skeletal muscle \\
\hline $\begin{array}{l}\text { MyHC IIx / Myosin-1 } \\
(\text { Myh1) }\end{array}$ & $\begin{array}{l}\text { Myosin Heavy Chain IIx / Myosin, heavy polypeptide 1, skeletal muscle, } \\
\text { adult }\end{array}$ \\
\hline NFAT (Nfat) & Nuclear factor of activated T-cells \\
\hline NKX-6.1 (Nkx6-1) & NK6 homeobox 1 \\
\hline PDYN (Pdyn) & Prodynorphin / Proenkephalin-B \\
\hline PGC-1a (Pgc1a) & Peroxisome proliferator-activated receptor $\mathrm{y}$ coactivator-1 alpha \\
\hline PI3K (Pik3) & Phosphatidylinositol 3-kinase \\
\hline PROX1 (Prox1) & Prospero homeobox 1 \\
\hline PSIP1 (Psisp1) & PC4 and SF2 interacting protein 1 \\
\hline PTBP2 (Ptb2b) & Polypyrimidine tract binding protein 2 \\
\hline RAB7 (Rab7) & RAB7, member RAS oncogene family \\
\hline RANBP2 (Ranbp2) & RAN binding protein 2 \\
\hline RBP & mRNA binding protein \\
\hline RNase & Ribonuclease \\
\hline RPL29 (Rp/29) & Ribosomal protein L29 \\
\hline RPS4L (Rps4) & Ribosomal protein S4-like \\
\hline RRM2B (Rrm2b) & Ribonucleotide reductase M2 B (TP53 inducible) \\
\hline SIL1 (Sil1) & $\begin{array}{l}\text { Nucleotide exchange factor SIL1 / Endoplasmic reticulum chaperone SIL1 } \\
\text { homolog (S. cerevisiae) }\end{array}$ \\
\hline SRSF1 (Srsf1) & Serine/arginine-rich splicing factor 1 \\
\hline SV2A (Sv2a) & Synaptic vesicle glycoprotein $2 a$ \\
\hline TET1 \& TET2 (Tet1/2) & Tet methylcytosine dioxygenase 1 and 2 \\
\hline VAChT (S/c18a3) & $\begin{array}{l}\text { Solute carrier family } 18 \text { (vesicular monoamine), member } 3 \text { / Vesicular } \\
\text { acetylcholine transporter }\end{array}$ \\
\hline YME1L1 (Yme1/1) & YME1-like 1 (S. cerevisiae) \\
\hline
\end{tabular}




\section{Molecules}

\begin{tabular}{ll}
\hline ACh & Acetylcholine \\
ADP & Adenosine di-phosphate \\
AMP & Adenosine mono-phosphate \\
ATP & Adenosine tri-phosphate \\
DNA & Deoxyribonucleic acid \\
RNA & Ribonucleic acid \\
mRNA & Messenger ribonucleic acid \\
miRNA & Micro ribonucleic acid \\
rRNA & Ribosomal ribonucleic acid
\end{tabular}

\section{Other abbreviations}

$\begin{array}{ll}\text { ALS } & \text { Amyotrophic lateral sclerosis } \\ \mathrm{CI} & \text { Confidence interval } \\ \text { ctrl } & \text { control } \\ \text { ER } & \text { Endoplasmic Reticulum } \\ \text { FF } & \text { fast, fatigable } \\ \text { FI } & \text { fast, intermediate } \\ \text { FR } & \text { fast, fatigue-resistant } \\ \text { GO } & \text { Gene Ontology } \\ \text { MDO } & \text { Mito-Dendra2-flox;Olig2-Cre } \\ \text { MN } & \text { Motor neuron; aNM, } \beta \text { MN, yMN } \\ \text { NMJ } & \text { Neuromuscular junction } \\ \text { run } & \text { voluntary wheel running } \\ \text { S } & \text { slow, fatigue-resistant } \\ \text { SD } & \text { Standard deviation } \\ \text { SMA } & \text { Spinal muscular atrophy } \\ \text { TA } & \text { Tibialis anterior }\end{array}$




\section{List of figures}

\section{Introduction}

Figure II.1 Segmental organization of spinal motor columns at cervical (A), brachial/lumbar (B) and thoracic (C) levels

Figure II.2 Schematic representation of the different types of motor neurons

Figure II.3 Summary of principal differences between motor neuron subtypes

Figure II.4 Morphological characteristics and spatial organization of motor neuron classes and subtypes

Figure II.5 The architecture of a neuromuscular junction: the synaptic interface between a branch of a motor neuron and muscle cells

Figure II.6 An Omics approach to decipher the molecular basis of exercise adaptation

Figure II.7 Scheme of the signaling pathways and transcriptional factors and co-regulators involved in the control of slow gene program and oxidative gene program

Figure II.8 Signaling pathways in hypertrophy and atrophy

Figure II.9 Exercise and insulin regulation of glucose transport

Figure II.10 Genes and proteins controlled by exercise at the vertebrate neuromuscular junction

\section{Material \& Methods}

Figure III.1 Strategy to genetically tag mitochondria in mouse motor neurons

Figure III.2 Conditional expression of the Dendra2 fluorescent protein in the mitochondria of spinal motor neurons

\section{Results}

Figure IV.1 Running performances among the animals

Figure IV.2 Fiber type distribution in the predominantly slow soleus muscle of sedentary and trained mice

Figure IV.3 Fiber type distribution in the fast-intermediate plantaris muscle of sedentary and trained mice

Figure IV.4 Soleus NMJs of sedentary and voluntary running MDO animals

Figure IV.5 Tibialis anterior NMJs of sedentary and voluntary running MDO animals.

Figure IV.6 3D reconstruction of soleus and tibialis anterior NMJs of sedentary and voluntary running animals enables precise measurements.

Figure IV.7 Upon exercise, the size of the post-synapse of soleus NMJs increases, while it decreases in tibialis anterior (TA) NMJs

Figure IV.8 The presynaptic compartment of soleus NMJs contains a large volume of mitochondria that increases with exercise

Figure IV.9 The presynaptic compartment of tibialis anterior (TA) NMJs contains a large number of mitochondria clusters, which decreases with exercise 
Figure IV.10 No modification of mitochondria cluster shape in the pre-synaptic NMJs of voluntary running mice.

Figure IV.11 Voluntary running exercise does not strikingly affect the relationship between the measured variables in the NMJs of soleus and tibialis anterior (TA) muscles

Figure IV.12 Accurate separation of the dorsal from the ventral region of the dissected spinal

Figure IV.13 Long-term voluntary running alters the gene expression profile of the soleus and the ventral spinal cord

Figure IV.14 Biological processes activated or repressed upon exercise training in the soleus muscle

Figure IV.15 Molecular functions activated or repressed upon exercise training in the soleus muscle

Figure IV.16 Exercise training mainly activated the centrosomal compartment in the soleus muscle

Figure IV.17 Exercise training mainly repressed activity of the mitochondrial compartment in the soleus muscle

Figure IV.18 Biological processes activated or repressed upon exercise training in the dorsal spinal cord

Figure IV.19 Molecular functions activated or repressed upon exercise training in the dorsal spinal cord

Figure IV.20 Exercise training mainly activated the nuclear compartment in the dorsal spinal cord

Figure IV.21 Exercise training mainly repressed activity of the mitochondrial compartment in the dorsal horn of the spinal cord

Figure IV.22 Biological processes activated or repressed upon exercise training in the ventral spinal cord

Figure IV.23 Molecular functions activated or repressed upon exercise training in the ventral spinal cord

Figure IV.24 Exercise training mainly activated the nuclear compartment in the dorsal spinal cord

Figure IV.25 Exercise training mainly repressed activity of the mitochondrial and endoplasmic reticulum compartments in the dorsal spinal cord

Figure IV.26 Common response of dorsal spinal cord, ventral spinal cord and soleus muscle to long-term endurance training

Figure IV.27 Twelve weeks wheel running induces changes in gene expression in the soleus muscle

Figure IV.28 Endurance training regulates the expression level of genes involved in synaptic processes: transmission, morphogenesis and mitochondrial organization

Table IV.1 Muscle fiber type distribution in trained and sedentary C57BL/6J mice

Table IV.2 Total number of fibers in muscles of trained and sedentary C57BL/6J mice

Table IV.3 Selected genes from the common response to training, which play a role in the regulation of transcription and splicing, in oxidation-reduction processes and in mitochondrial membrane organization 


\section{Abstract}

Axon terminals are likely the compartments of highest energy demand in the particularly active and polarized cells that are motor neurons. Paradoxically, although the particularly high-energy demand of motor neurons could make them more prone to energetic stress, endurance exercise appears to mediate the strengthening of the neuromuscular synapse. Moreover, tight regulation of mitochondrial biogenesis is of utmost importance for meeting elevated energy demands in neurons, and thus mitochondrial plasticity may adapt pre-synaptic motor neuron metabolic properties to increased energetic stress. While mitochondrial biogenesis and function have been extensively studied in muscle, much less is known regarding mitochondrial network remodeling at the neuromuscular junction upon exercise. In this study, exerciseinduced changes at the neuromuscular junction were directly observed and measured using mice expressing genetically tagged mitochondria specifically in motor neurons, followed by 3D-reconstruction of synaptic structures. In addition, RNA sequencing of muscle as well as dorsal and ventral spinal cord samples from both control and long-term voluntary trained mice, enabled the identification of genes which expression was regulated upon physical activity. Thus, the present study provides insights on the regulations in gene expression that may modulate mitochondrial biogenesis and stress pathways upon physical exercise and how these changes may impact motor neuron function and ultimately mediate exercise beneficial effects. 


\section{Introduction}

"The human body has more than 300 bilateral pairs of muscles containing more than 100 million muscle fibers, which are innervated by more than 120,000 motor neurons in the spinal cord alone" (Kanning et al., 2010). A well-defined organization of the entire motor system is an essential requirement in order to maintain proper body posture, elicit complex motor behaviors and allow performance of fine and precise movements. The architecture and functional connections between the numerous elements of the motor network are acquired during development (see Stifani, 2014, for review). All components of the motor system, including motor neurons and muscle fibers, not only follow a precise scheme of distribution throughout the brain and the spinal cord, but also mature into multiple cellular identities (see Lu et al., 2015, for review). This diversity is fundamental for the regular function of the motor system as well as for its adaptation to different usage or health states.

\subsection{The neuromuscular system}

The generation of all behavior programs in the central nervous system and their conversion into body movements is enabled by a specialized class of neurons: the motor neurons (MNs). Motor commands are generated in the pre-motor and primary motor cortex by upper MNs and transmitted through descending motor pathways to spinal MNs (lower MNs), which directly synapse on limb skeletal muscle fibers. The spinal MNs receive and integrate these supra-spinal inputs together with intra-spinal and sensory inputs to control the contraction of muscles and thus enable locomotion. Sherrington therefore described the spinal MNs as the "final common path" of motor control to skeletal muscle (Sherrington, 1904). The connections between MNs and muscles are organized in three architectural levels, where the localization of the MNs in the spinal cord correlates with the position of their target muscle in the body. Along the rostro-caudal axis of the spinal cord, MNs are arranged into motor columns supplying distinct muscle groups (figure II.1.; Romanes, 1951; Landmesser, 1978; Vanderhorst \& Holstege, 1997). Further, each muscle is innervated by a distinct and 


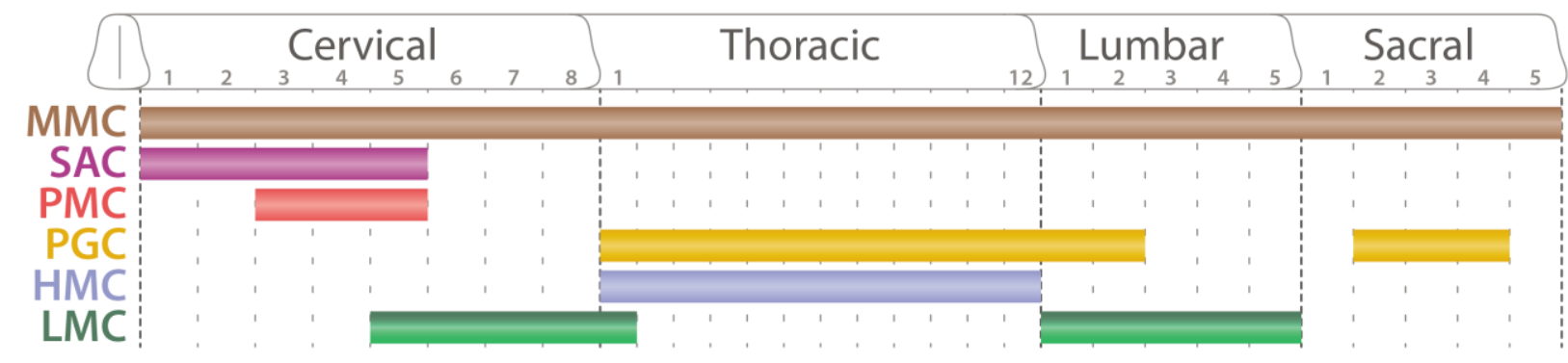

A
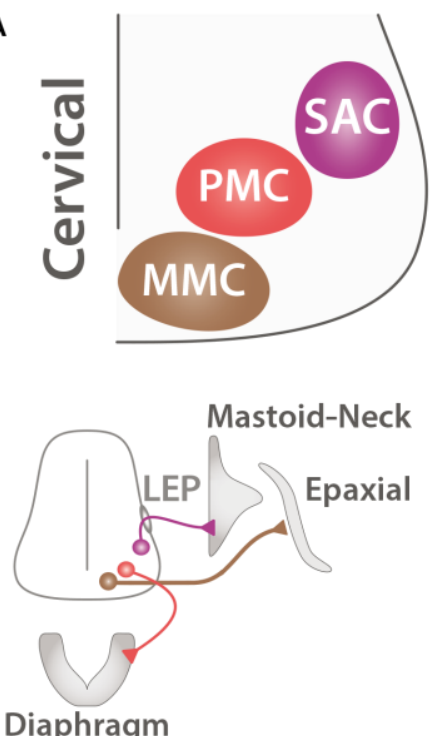

B
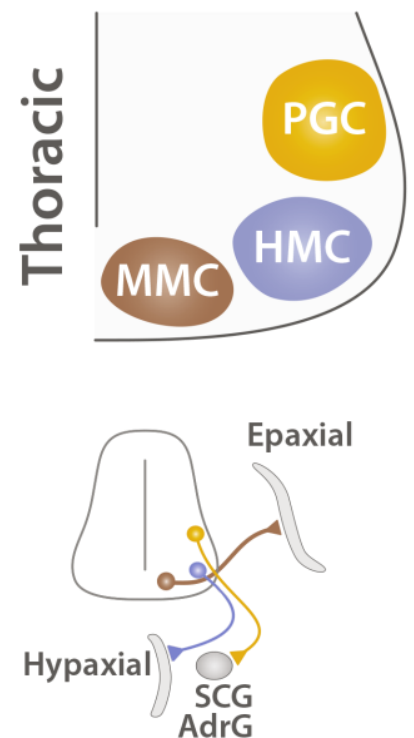
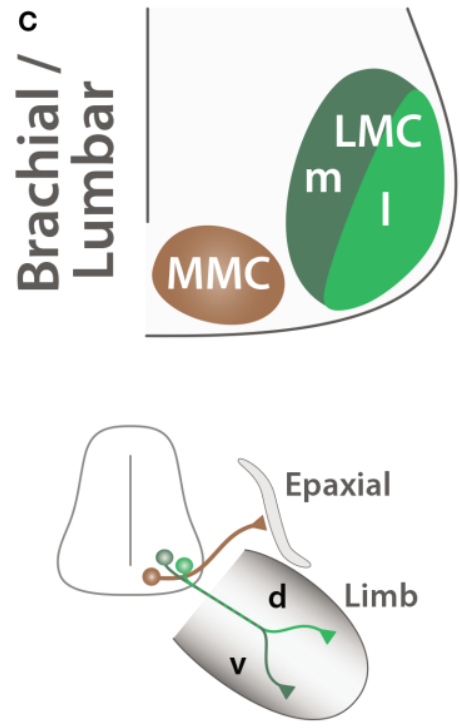

Figure II.1. Segmental organization of spinal motor columns at cervical (A), brachial/lumbar (B) and thoracic (C) levels. The medial motor column ( $\mathrm{MMC}$, brown) is present all along the rostrocaudal axis; MMC MNs are located medially and connect to the axial musculature (Epaxial) (A-C). The spinal accessory column (SAC, purple) is restricted to the five first cervical segments (C1-C5); SAC MNs exit the CNS via the lateral exit point (LEP) and connect to mastoid and neck muscles (A). The phrenic motor column (PMC, red) is confined between C3 and C5 PMC MNs have an inter-mediolateral position and connect to the diaphragm (A). The preganglionic column (PGC, orange) extends through the thoracic segments until the second lumbar segments (L2) as well as between sacral segments S2 and S4; PGC MNs are positioned dorso-laterally and innervate the sympathetic chain ganglia (SCG) and the chromaffin cells of the adrenal gland (AdrG) (B). The hypaxial motor column (HMC, blue) is exclusive to the thoracic segment whereas the lateral motor column (LMC, green) is located at limb levels: brachial (C5-T1) and lumbar segments (L1-L5); HMC MNs are located in the medio-lateral region and connect to the body wall and intercostal muscles (Hypaxial) (B). LMC MNs (green) are divided into two divisions medial (m, dark green) and lateral (I, light green); LMCm MNs connect to the ventral (v) part of the limb whereas LMCI MNs innervate the dorsal (d) region (C). (adapted from Stifani, 2014) 
specific cluster of spinal MNs forming a discrete nucleus, termed motor pool (McHanwell and Biscoe, 1981). Finally, within a motor pool, a single MN and the multiple muscle fibers it innervates constitute a motor unit, the functional unit of the motor system (Buchthal and Schmalbruch, 1980).

\subsubsection{Spinal motor neurons and motor neuron diversity}

The cell bodies, or somata, of the lower MNs are located in lamina IX in the ventral horn of the spinal cord (Rexed, 1952). From here, their axons exit the CNS and project alongside sensory and sympathetic axons via the peripheral nerves to eventually synapse onto skeletal muscle fibers. MNs belong to different classes, defined by the nature of their target fibers. Gamma MNs ( $\mathrm{YMNs}$ ) connect intrafusal muscle fibers, while alpha MNs (aMNs) innervate extrafusal muscle fibers, and the less well characterized beta $M N s$ ( $\beta M N s)$ innervate both intra- and extrafusal fibers (figure II.2.; Manuel and Zytnicki, 2011).

The different muscle fiber types are described in more details in section 2.1.3. yMNs do not contribute to overall muscle force generation, but are essential for generating muscle tone and for maintaining muscle proprioception during movements. They can be subdivided into dynamic and static yMNs based on the discharge they elicit at the spindle sensory endings (Bessou et al., 1962). The more abundant aMNs can be further divided into four subtypes, namely slow (S), fast resistant (FR), fast intermediate (FI) and fast fatigable (FF) MNs (Burke et al., 1973). This classification is based on particular combinations of physiological and histochemical characteristics of the motor unit supplied by MNs (Burke et al., 1973). The MNs associated with these motor units appear to form a continuous population based on electrophysiological properties alone (Zengel et al., 1985). However, the combination of certain biophysical parameters together with morphological and molecular characteristics allowed the precise identification of motor unit type affiliation of individual MNs.

On one end of the spectrum, S MNs have small cell bodies and axons. S MNs also have a specific structure of the dendritic tree, with less profuse branching but more dendritic bundles compared to fast MNs (Cullheim et al. 1987; Westerga and Gramsbergen, 1992). At the other end, FF MNs are larger in size, with large-diameter 


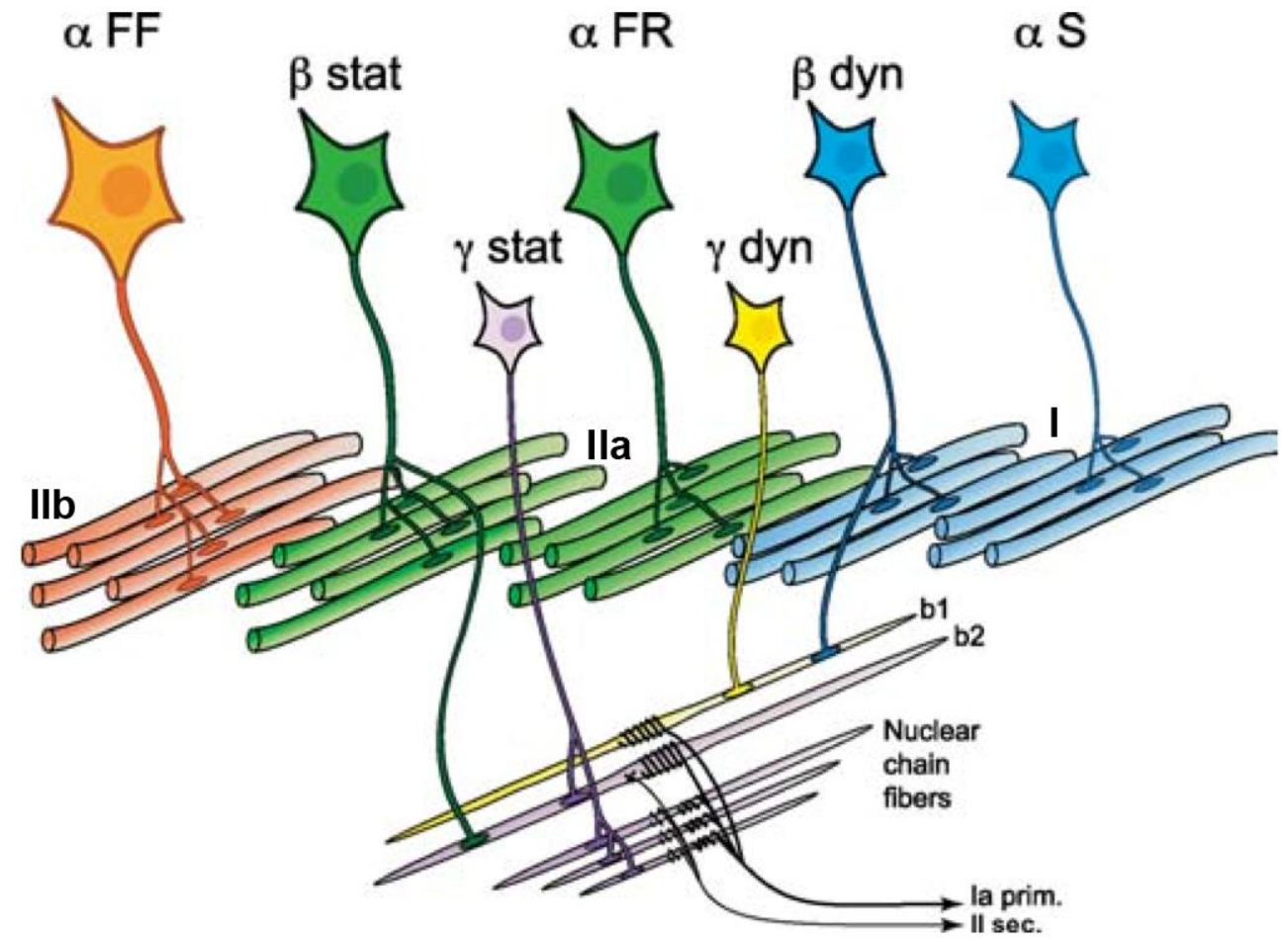

Figure II.2. Schematic representation of the different types of motor neurons. FF-type aMNs are the biggest MNs (in terms of soma size and axon diameter), and innervate a large number of type Ilb extrafusal muscle fibers. FR aMNs are slightly smaller and innervate type lla extrafusal muscle fibers. S-type aMNs are the smallest of the aMNs, they innervate fewer type I muscle fibers. BMNs are skeleto-fusimotor: they innervate both extrafusal and intrafusal muscle fibers. Static $\beta M N s$ innervate either type Ila or Ilb extrafusal fibers and the intrafusal bag2 fiber. Dynamic $\beta M N s$ innervate type I extrafusal muscle fibers and the intrafusal bag1 fiber. $y M N$ s innervate exclusively intrafusal muscle fibers and are the smallest of the MNs. Static YMNs innervate the intrafusal bag2 fiber and/or the nuclear chain fibers. Dynamic yMNs innervate the intrafusal bag1 fiber. Note that in a muscle, the various types of large extrafusal muscle fibers are mingled together and organized in a mosaic, while the intrafusal muscle fibers are much smaller and are ensheathed in the spindle capsule. Primary and secondary endings of the spindle encode parameters of the muscle stretches that are sent to the central nervous system via afferent fibers la and II. (from Manuel and Zytnicki, 2011) 
fast-conducting axons, more axonal and dendritic branches and more neuromuscular terminals (Celichowski et al., 1996). Due to their smaller size, S MNs have higher input resistance combined with a low rheobase (minimum amount of current required to elicit an action potential). S MNs therefore require less synaptic activation to initiate action potentials. Hence, upon increasing the presynaptic input to a motor pool, the more easily excitable small MNs reach threshold before large MNs (Bakels and Kernell, 1993; Gardiner, 1993). S MNs show repetitive firing (tonic discharge) that can persist even after the presynaptic excitatory drive has ceased (Tansey and Botterman, 1996), while FF MNs show a phasic discharge. Another physiologic difference between aMN subtypes is that FF MNs have a shorter post-spike afterhyperpolarization than $\mathrm{S} M N s$, so that the firing frequency of each subtype is speed matched to the contractile frequency of the target muscle fiber (Bakels and Kernell, 1993; Gardiner, 1993). Different expression profiles of ion channels in the MN types probably underlie the specificity of their physiological properties, although their identities remain to be defined. The principal differences between $\mathrm{MN}$ subtypes are summarized in the review from Kanning et al., 2010 (figure II.3.).

MNs are the only cholinergic neurons in the ventral spinal cord, at the exception of a few interneurons lateral to the central canal, the medial partition neurons, which also release the neurotransmitter acetylcholine (ACh). Thus, their identification in spinal cord sections is usually done by immunohistochemistry against the ACh synthetizing enzyme ChAT (choline acetyltransferase; see figure IV.12.B. ChAT staining paraffin sections) or the ACh transporter VAChT (vesicular acetylcholine transporter). Recent studies have begun to identify putative molecular markers for fast and slow MNs. Fast MNs can be identified at the postnatal stage by their expression of DLK1 (Delta-like homolog 1) and at the adult stage by CGRP-1 (calcitonin gene-related peptide 1), Chondrolectin and MMP9 (matrix metalloproteinase 9), while slow MNs express SV2A (synaptic vesicle glycoprotein 2a) and SIL1 (endoplasmic reticulum chaperone SIL1 homolog) (Mueller at al., 2014; Forsgren et al., 1993; Piehl et al., 1993; Enjin et al., 2010; Kaplan et al., 2014; Chakkalakal et al., 2010; Filézac de L'Etang et al., 2015). Finally, aMNs have a wellestablished differential vulnerability towards aging and degenerative conditions (figure II.3.; Hegedus et al., 2007; Hegedus et al., 2008). For instance, in the deadly human motor neuron disease amyotrophic lateral sclerosis (ALS), FF MNs invariably 


\begin{tabular}{|c|c|c|c|c|}
\hline \multirow[b]{2}{*}{ Motor unit characteristic } & \multicolumn{4}{|c|}{ Motor neuron subtype and class } \\
\hline & FAST & SLOW & ALPHA & GAMMA \\
\hline Target fiber & Fast $(\Pi \mathrm{a} / \mathrm{b} / \mathrm{x})$ & Slow (I) & Extrafusal & Intrafusal \\
\hline Presence in different motor pools & Variable & Variable & All & Most \\
\hline Cell body size & Larger & Smaller & Larger & Smaller \\
\hline Dendrite length & Similar & Similar & Similar & Similar \\
\hline Dendrite branching & More & Less & More & Less \\
\hline Direct Ia proprioceptive input & Most & Most & Most & None \\
\hline Synaptic density on proximal dendrites & Similar & Similar & Higher & Lower \\
\hline Synaptic density on distal dendrites & Lower & Higher & Higher & Lower \\
\hline C-type boutons on soma & More & Less & Present & Absent \\
\hline EPSP magnitude from Ia afferents & Smaller & Larger and longer & Greater & None \\
\hline Membrane input resistance & Smaller & Larger & Larger & Smaller \\
\hline Order of recruitment & Late & Early & Size dependent & - \\
\hline Firing behavior & Phasic & Tonic & Subtype dependent & Subtype dependent \\
\hline Bistable behavior & No & Yes & Yes & Maybe \\
\hline Axon conduction velocity & Faster & Slower & Faster & Slower \\
\hline Post-spike after-hyperpolarization & Shorter & Longer & Subtype-dependent & Varied, debatable \\
\hline Axonal recurrent collaterals & More & Less & Yes & No \\
\hline Motor unit size (innervation ratio) & Larger & Smaller & Larger & Smaller \\
\hline NMJ morphology & $\begin{array}{l}\text { Larger, more } \\
\text { complex }\end{array}$ & $\begin{array}{l}\text { Smaller, less } \\
\text { complex }\end{array}$ & Deep synaptic folds & Shallow synaptic folds \\
\hline Synaptic vesicle density in terminals & Lower & Higher & - & - \\
\hline Affected in ALS & Early & Late & Yes & Unclear \\
\hline Affected in aging & Early & Late & Yes & No \\
\hline Molecular correlates & - & SV2a (adult) & NeuN, Hb9::GFP ${ }^{+}$ & $\begin{array}{l}\text { Err3, Gfr } \alpha 1, \text { SDH }{ }^{\text {hi }} \text {, } \\
\text { TrkC }\end{array}$ \\
\hline
\end{tabular}

Figure II.3. Summary of principal differences between motor neuron subtypes. The table summarizes the functional and molecular differences discussed in the text between fast and slow, and between alpha and gamma, motor neurons. The descriptors are for some comparisons relative trends rather than absolute rules and apply only within a given pairwise comparison, not across the whole table. This is because absolute values vary between species and at different ages. Abbreviations: ALS, amyotrophic lateral sclerosis; EPSP, excitatory postsynaptic potential; NMJ, neuromuscular junction. (from Kanning et al., 2010) 
undergo degeneration first, followed by FR MNs, whereas $S$ MNs are well preserved until late stages of the disease (Frey et al., 2000; Pun et al., 2006). Some of the molecular markers for MN types, such as MMP9 and SIL1 have been implicated in contributing to the differential susceptibility of MN types to disease.

The diversity of aMNs in terms of morphological, electrical and molecular properties is presumably further matched by different metabolic properties. Vertebrate MNs have a large soma, compared to other spinal neurons and are highly polarized cells with an extensive dendritic profile and a considerably long axon extending far away from the cell body (Mayhew and Momoh, 1974). Moreover, the MNs innervating the postural skeletal muscles are constantly recruited, meaning that they continuously fire and that there is regular neurotransmitter release at the axon terminals, also termed endplates. Thus energy demand in $\mathrm{MNs}$ is high in the soma but also in cell compartments very distant from the cell body. However, MN metabolic diversity still remains largely unknown.

\subsubsection{Skeletal muscles and skeletal muscle diversity}

To understand $\mathrm{MN}$ biology, one must understand muscle fiber diversity. Skeletal muscles are anchored by tendons to bone and are used to effect skeletal movements. Proper function of skeletal muscles is ensured by the combined action of intrafusal and extrafusal muscle fibers, or myofibers. On the one hand, the short and thin intrafusal muscle fibers that are dually innervated by yMNs and sensory neurons are implicated in maintaining muscle proprioception and in generating muscle tone (Kuffler et al. 1951). A few intrafusal fibers innervated by both sensory neurons and YMNs, wrapped by a capsule derived from terminal Schwann cells of sensory axons, constitute a muscle spindle. The spindles act as stretch-sensitive mechanoreceptors able to monitor changes in muscle length. On the other hand, force results from the contraction of the extrafusal muscle fibers innervated by aMNs (figure II.2.; Manuel and Zytnicki, 2011). The role of skeletal muscles is to generate force for maintaining body posture against gravity, as well as for moving the skeleton. In physiological conditions, the same muscle is usually used for various tasks. For example, the Soleus muscle displays a continuous low-intensity activity for maintaining the posture, whereas the Tibialis anterior exhibits repeated submaximal 
contractions for driving locomotion. But Tibialis anterior is also recruited for powerful actions such as jumping or kicking, which needs fast and strong maximal contractions. To allow this flexibility, most skeletal muscles are heterogeneous in their fiber composition (for extended review see Schiaffino and Reggiani, 2011).

Muscle fibers are traditionally classified as fast-twitch or slow-twitch fibers, based on their time to peak tension or half-relaxation time (Close, 1967). The power and velocity of contraction of a fiber correlates with its myosin heavy chain (MyHC) isoform composition. In extrafusal fibers of mammalian limbs, four different adult MyHC isoforms can be expressed that range from "slow - low force" to "fast - high force": I, Ila, IIx, IIb. These profiles are not exclusive as some fibers co-express two isoforms together, generating hybrid fibers: I-Ila, Ila-Ilx or Ilx-Ilb (Bottinelli et al., 1994). The myosin types of fibers also determine their size, with a smaller mean diameter in slow fibers compared to fast fibers (Delp and Duan, 1996). Given the central role of myosin in determining the contractile performance and ATP consumption of fibers, and the convenience of myosin expression profile determination by immunostaining, $\mathrm{MyHC}$ is the marker of preference to determine the type of a fiber. However, this simplification masks the actual complexity of fiber identities. Indeed, muscle fibers exhibit a large panel of physiological and mechanical properties including membrane excitability, intracellular calcium signaling, contractile response, and energy supply (Pette and Staron, 1990). Fiber types results from the preferential combinations of all these specific properties in order to match with the functional requirements of the fibers, for instance energy production with energy consumption or calcium release with calcium uptake (Schiaffino and Reggiani, 2011).

In response to $\mathrm{ACh}$ binding its receptor at the neuro-muscular synapse, the membrane of the muscle fibers depolarizes, leading to muscle contraction. The muscle fiber membrane, or sarcolemma, of fast fibers shows higher ionic conductance at rest and during activity than slow fibers (Bretag, 1987; Milton and Behforouz, 1995; Tricarico et al., 2006). As a consequence, fast fibers have a rapid repolarization and a short refractory period, when slow fibers are able to compensate the accumulation of ions induced by continuous stimuli. These differences in transmembrane ionic fluxes directly impacts intracellular calcium signaling (Baylor and Hollingworth, 2003). Compared with slow-twitch, fast-twitch fibers are characterized by the generation of fast and large calcium transients, which work 
together with a responsive contractile machinery (Carroll et al., 1997). The calcium kinetics and calcium sensitivity of the myofibrils controls the availability of the actin binding sites and thus plays a switch ON-switch OFF role on the contraction mechanism. The myofibrillar cytoskeleton scaffold differs in fast and slow muscle fibers, consistent with the properties of the contractile machinery and specifically correlates with the mechanical tension generated by contraction (Pette and Staron, 1990). Slow fibers have longer and more extensible titin and nebulin isoforms (sarcomeric giant proteins), as well as longer actin filaments resulting in longer sarcomeres. Thus continuously active slow fibers can withstand passive elongation with less mechanical resistance. Moreover, slow fibers have thicker Z-discs and Mband, a feature that is probably related to the ability to withstand active force (Schiaffino et al., 1970). As a consequence, maximal force is higher in fast fibers, but active forces are generated for longer time in slow fibers.

These mechanical properties have a counterpart in energy metabolism and the metabolic diversity among muscle fibers, which directly correlates with their contractile activity. Slow fibers contract more slowly and generate less mechanical power but also spend less ATP, particularly in relation to tension development (Bottinelli et al., 1994). On the other hand, fast fibers can produce higher mechanical power and contract more quickly, but have a higher ATP expenditure. In addition to differences in ATP expenditure, fast and slow fibers also diverge in their strategies for ATP regeneration. Different enzymatic mechanisms between slow and fast fibers cause greater activation of glycogenolysis in fast fibers, while resulting in greater glucose uptake and beta-oxidation in slow fibers, due to the greater mitochondrial density (Schiaffino et al., 1970; Peter et al., 1972). Diversity is also present in the regulation of mitochondrial activity between slow and fast fibers, with a more effective stimulation due to ADP in fast fibers, and a stimulation due to creatine that is more effective in slow fibers (Spamer and Pette, 1977). Importantly, once mitochondrial respiration and ATP regeneration are activated, consumption is covered by regeneration in slow fibers. Such condition of complete energy balance is never achieved in fast fibers. Finally, based on electrical activity and ATP consumption during contraction, the overall daily energy expenditure in a slow oxidative muscle was estimated to be 10 to 20 times greater than that of a fast glycolytic muscle (Schiaffino and Reggiani, 2011). This implies another important diversity between 
slow and fast fibers: slow fibers are able to take up 20 times more substrates (glucose, fatty acids, amino acids and lactate) than fast fibers. At the scale of the whole organism, fast and slow muscles contribute differently to the global energy economy.

It is appreciated that under physiological conditions, muscle fiber types show a continuum of maximum force, twitch speed, and endurance (Kernell et al. 1999). On one side of the spectrum are the historically named "red" muscles like the Soleus muscle for example. They are constituted by slow-twitch fibers, rich in myoglobin and oxidative enzymes and specialized for low-intensity continuous activity. On the other side are the so-called "white" muscles, which Tibialis anterior is a typical example. They are composed of fast-twitch fibers, characterized by glycolytic metabolism and specialized for short and strong contractile performance.

\subsubsection{Organization of the motor system and types of motor units}

A single $\mathrm{MN}$ and the multiple muscle fibers it innervates constitute a functional unit: the motor unit (Lidell and Sherrington, 1925; Buchthal and Schmalbruch, 1980). There is a high degree of correlation between the different parts of a same unit. Two conditions are necessary to establish a full congruence between $\mathrm{MN}$ and muscle fibers. First, the MN connects a bunch of muscle fibers with similar, if not identical, structural and functional properties (Edstrom and Kugelberg, 1968). The second condition is that muscle fiber electrophysiological properties are compatible with the discharge pattern of the motor neuron (Bakels and Kernell, 1993; Schiaffino and Reggiani, 2011). Knowing the electrophysiological properties of a MN allows predicting with high accuracy the type of motor unit it forms and the muscle fiber types it innervates (Gardiner, 1993). The establishment of the functional coupling of MNs and the respective muscle fibers they innervate led to the classification of motor units in different types: a $S M N$ innervating type I muscle fibers constitute a slowtwitch fatigue-resistant (S) motor unit, FR MN with type lla fibers is a fast-twitch fatigue-resistant (FR) motor unit, $\mathrm{FI} \mathrm{MN}$ and type IIx fibers is a fast-twitch intermediate (FI) motor unit, and finally a FF MN connecting type llb fibers constitute a fast-twitch fatigable (FF) motor unit (Burke et al., 1973; McDonagh et al., 1980 and figure II.4). 

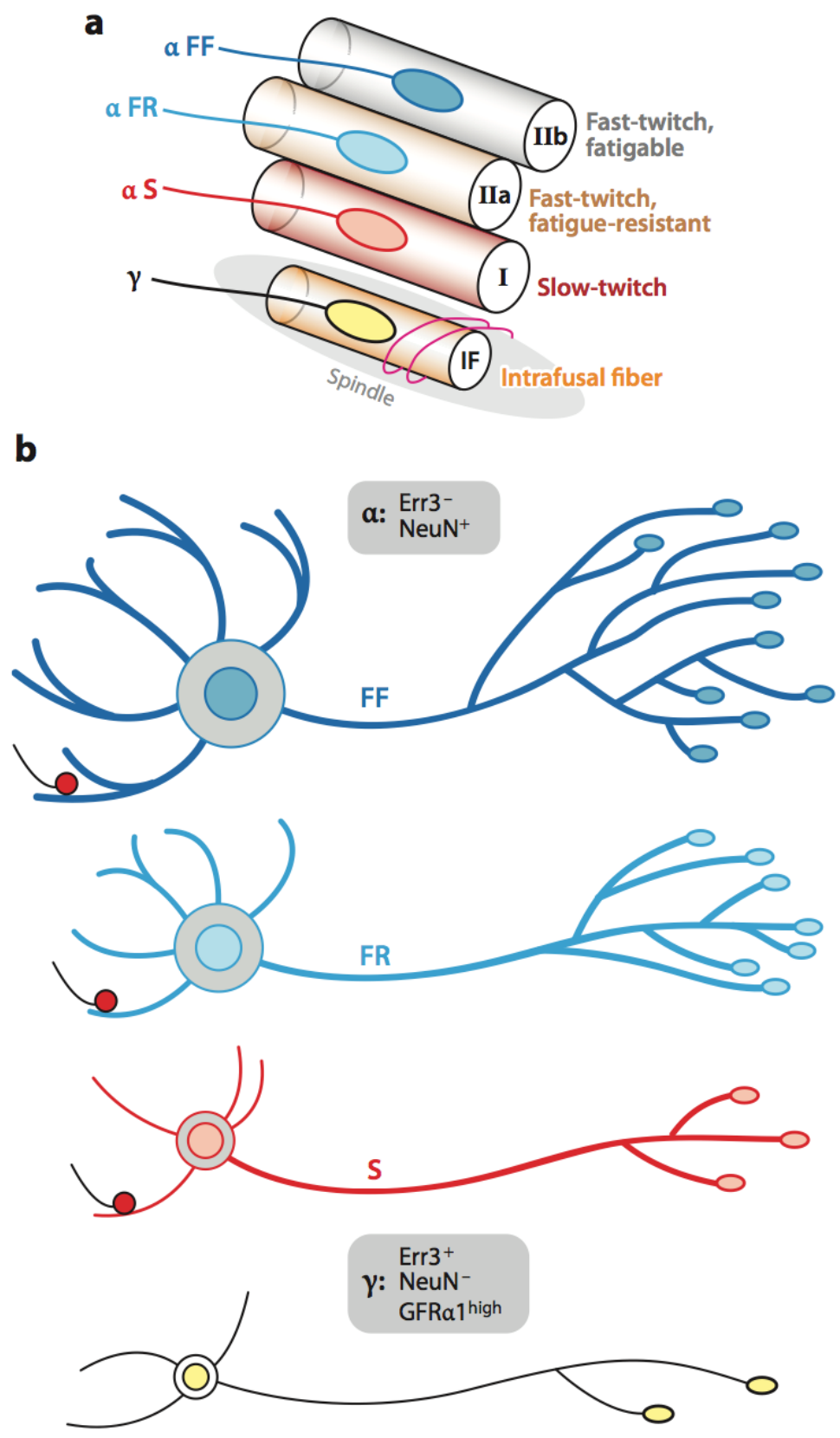

Figure II.4. Morphological characteristics and spatial organization of motor neuron classes and subtypes. (a) Distinct motor units within skeletal muscle. aMNs innervate extrafusal muscle fibers to form three subtypes of motor unit: Slow-twitch (S) units control Type I fibers, fast-twitch fatigueresistant (FR) units control Type Ila fibers, and fast-twitch fatigable (FF) units control Type Ilb/x fibers. Intrafusal (IF) muscle fibers are innervated by YMNs and also by $\beta M N s$ (not shown). (b) The size and morphological complexity of aMNs diminish progressively from FF through FR to S motor units. Most aMNs receive direct la innervation from VGLUT1+ (vesicular glutamate transporter 1) proprioceptive sensory neurons (red filled circles). yMNs are smaller still and do not receive la innervation. At postnatal stages, $\alpha$ - and $\gamma-M N$ cell bodies can be distinguished by their size, connectivity, and the indicated molecular markers. (from Kanning et al., 2010) 
Motor unit functional diversity is a necessity for the precise and smooth execution of movements because it facilitates the automated gradation and taskdependent application of force by each muscle (Kernell, 2003). Two distinct mechanisms regulate the gradation of muscle force: the alteration of the $\mathrm{MN}$ firing pattern and the recruitment of additional motor units (Milner-Brown et al., 1973a; Milner-Brown et al., 1973b). The hierarchy of motor unit recruitment is based on the Henneman "size principle" from which it follows that during muscle contraction S MNs reach activation threshold first, while large $\mathrm{MNs}$ are activated last. As a consequence, during postural tasks such as standing, or activities requiring low force levels over short periods of time such as normal walking, only the slowest motor units are active (Zajac and Faden, 1985). As a corollary, the later-recruited fast motor units are mainly employed in short-lasting bouts of forceful contraction such as jumping. This control provides a greater economy of force generation during most normal functional daily activities (De Luca and Contessa, 2012). Whether MN firing rate coding or motor unit recruitment is the dominating mechanism at a given level of muscular activation can be predicted from a principle of minimum-energy expenditure in which motor units are activated according to their energy requirements (Hatze and Buys, 1977). The recruitment begins with units of smallest energy expenditure (S units), followed by intermediate units (FR and FI units), the FF units only being called in when almost all of the intermediate ones have been activated. Recruitment dominates at low force levels, while at higher forces rate coding becomes increasingly more important (Hatze and Buys, 1977).

At a higher level of organization, all the motor units of the same muscle form a motor pool (McHanwell and Biscoe, 1981). Patterned contraction of several muscles (e.g. synergistic or antagonistic) is critical for eliciting accurate movements. Distinct classes of spinal premotor interneurons coordinate motor pool activities driving different muscle groups (Goulding, 2009). The heterogeneity of motor units is also reflected by systematic differences in their susceptibility to degenerative conditions and aging (Kanning et al., 2010 and figure II.3.). In motor neuron diseases such as amyotrophic lateral sclerosis (ALS) or spinal muscular atrophy (SMA), FF motor units degenerate early, whereas $S$ motor units are preserved until a late stage of the disease. Despite the variety of neuromuscular diseases, it now established that an increase in muscle use by the practice of physical exercise is beneficial in 
degenerative conditions, including motor neuron diseases (Grondard et al., 2005; Deforges et al, 2009; Anziska and Sternberg, 2013).

\subsubsection{The neuromuscular junction}

In the motor system, MNs and muscle fibers directly communicate through a unique synapse, namely the neuromuscular junction (NMJ). During muscle use, the excitation of the MNs elicits a precise sequence of events, which in turn leads to the contraction of the connected fibers. First, a given spinal MN receiving suprathreshold input fires action potentials that pass down the axon and axon terminal branches to the motor endplates. As a result of the MN membrane depolarization, vesicles containing the neurotransmitter fuse with the presynaptic membrane. The ACh released in the synaptic cleft then binds its receptors (AChRs) on the postsynaptic membrane (sarcolemma). The activation of the AChRs initiates a cascade leading to the depolarization of the sarcolemma. This allows the attachment of myosin heads to the thin actin filaments, which can finally slide over the thick myosin filaments resulting in muscle contraction (Schiaffino and Reggiani, 2011). At the same time, the acetylcholinesterase (AChE) released from the nerve in the synaptic cleft, hydrolyzes the neurotransmitter $\mathrm{ACh}$ to terminate the synaptic transmission (figure II.5). NMJs are subject to mechanical stress arising from muscular contraction and the efficacy of the system is based on the efficiency and reliability of this neuromuscular connection. In the mammalian NMJ, both the amount of ACh released at each nerve impulse and the number of subsequently activated AChR exceed the amount required to trigger sarcolemma depolarization. This allows a margin of safety to guarantee neuromuscular transmission under multiple conditions ranging from fast high-frequency bursts to prolonged low-frequency activation of muscles (Wood and Slater, 2001; Meriney and Dittrich, 2013).

NMJs exhibit certain diversity to coordinate $\mathrm{MN}$ and muscle activities and maintain the homogeneity of the motor unit. NMJs are "giant" synapses, which areas varie in proportion to the size of the muscle fiber. Absolute areas of endplates progressively increase from type I, Ila, IIx, to Ilb fibers; however, when normalized for fiber diameter, the areas of nerve terminals are largest in type I fibers (Prakash et al., 1996). Further, the fast and slow motor nerve terminals also differ in synaptic vesicle 


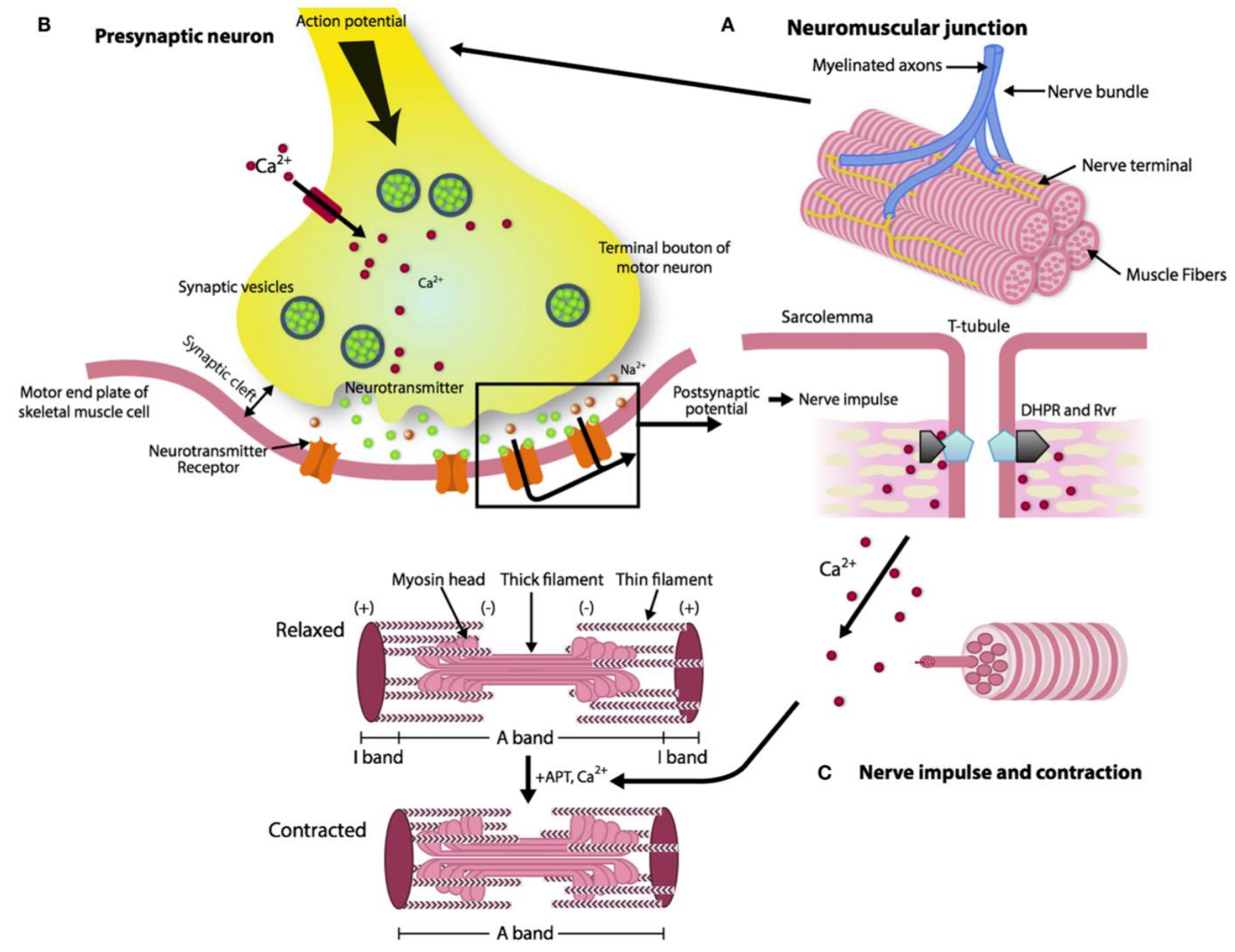

Figure II.5. The architecture of a neuromuscular junction: the synaptic interface between a branch of a motor neuron and muscle cells. (A, B) The neuromuscular junction is composed of three elements: pre-synaptic (motor nerve terminal), intra-synaptic (synaptic basal lamina), and postsynaptic component (muscle fiber and muscle membrane). When an action potential reaches the motor nerve terminal, voltage-dependent calcium channels open allowing calcium to enter the neuron and trigger the delivery of acetylcholine (ACh) in the synaptic cleft. (C) Acetylcholine triggers nicotin acetylcholine receptors ( $\mathrm{nAChR}$ ) located in the sarcolemma to produce an action potential, which in turn, activates voltage-gated dihydropyridine receptors (DHPRs) located in the sarcolemma and by induction, ryanodine receptors (RyRs). Of note, the post-synaptic membrane presents folds that expand its area. Calcium released from the sarcoplasmic reticulum through the RyRs binds to troponin $\mathrm{C}$ and allows cross-bridge cycling and force production. (from Gonzalez-Freire et al., 2014) 
dynamics. At the onset of stimulation, transmitter release is greater in fast fibers than in slow fibers (Reid et al., 1999). Accordingly, the amplitude of the muscle action potential is greater in fast fibers (Wood and Slater, 1997), which also have higher density of postsynaptic voltage-gated sodium channels within their postsynaptic folds (Milton et al., 1992). Finally, the content of AChE in slow fibers is about one-fourth of that in fast fibers and changes in relation to electrical activity and load (Pregelj et al., 2007). Taken together, the pre- and postsynaptic specializations are likely aimed to achieve a greater safety of transmission in NMJs of fast motor units and to resist synaptic depression during prolonged repetitive stimulation in NMJs of slow motor units (Schiaffino and Reggiani, 2011).

The transmission of the signal from the $\mathrm{MN}$ to the muscle at the NMJ requires presynaptic mechanisms including membrane depolarization and vesicle cycling (exocytosis and endocytosis) (Takamori, 2012). These processes involve ion channels to produce large fluxes of ions as well as a variety of enzymes to maintain metabolite production and recycling at the neuronal terminal. Thus, MNs require protein synthesis and turnover very far away from the cell body. For this reason, MNs possess local mechanisms for control of RNA translation to allow synthesis of new proteins in a spatially and temporally restricted manner (Liu-Yesucevitz et al., 2011). mRNA binding proteins (RBPs) have emerged as one of the major mechanisms to help neurons meeting the challenge of preventing synthesis of synaptic proteins during mRNA transport, yet quickly allowing synthesis upon demand in response to synaptic activity (Martin and Ephrussi, 2009). RBPs, microRNAs (miRNA) and/or ribosomal subunits reversibly aggregate in the cytoplasm to form RNA granules. The RNA granules work in concert with motor proteins and microtubules to regulate i) the distribution, transport and storage of mRNA transcripts throughout the neuron, ii) the translation of mRNA transcripts by either the sequestration of specific transcripts (repression) or the oligomerization of translation factors (activation), and iii) the regulation of mRNA degradation (Kiebler and Bassell, 2006; Thomas et al., 2011). A deficiency in the mechanisms of protein synthesis at the $M N$ terminals would greatly deteriorate its ability to release neurotransmitter vesicles. Synaptic transmission at the NMJ is impaired in different neuromuscular diseases and mutations in RBPs in particular are frequently associated with motor neuron diseases (Liu-Yesucevitz et al., 2011). Thus, a better understanding of these processes is essential to fully 
comprehend NMJ function in healthy conditions, NMJ malfunction in disease conditions, as well as NMJ adaptation to increased synaptic activity as it occurs during physical exercise.

\subsection{Neuromuscular adaptations to exercise}

As early as the Greco-Roman period, exercise and proper nutrition were known to improve mental and physical health: "It is exercise alone that supports the spirits, and keeps the mind in vigor" - Marcus Tullius Cicero. The ideal association of a healthy mind in a healthy body remained largely celebrated in the modern times: "True enjoyment comes from activity of the mind and exercise of the body; the two are ever united." - Wilhelm von Humboldt. It is thus well known that the organism is able to undergo different changes in response to increased physical activity, which result in improved body condition and cognitive performances. The adaptive capabilities of the system to both internal and external stimuli, such as regular physical activity, are termed "plasticity".

\subsubsection{Whole-body adaptations to exercise}

It is well established that physical activity not solely impacts muscles, but has multiple and various benefits on general health and performance. The systemic effects of exercise include greater general body fitness in healthy subjects and a better quality of life in older adults that results in increased average life expectancy (Mitchell and Barlow, 2011; Hollmann et al., 2007). Exercising was shown to delay typical age-associated impairments by reducing sarcopenia and increasing bone density, which are very important for the prevention of falls and osteoporosis in the elderly (Hollmann et al., 2007). Increased muscle use also reduces inflammation by the positive regulation of the immune system and prevents development of many chronic diseases (Perdersen and Hoffman-Goetz, 2000; Handschin and Spiegelman, 2008). It ameliorates glucose metabolism and gastro-intestinal function, which are severely impaired in type II diabetes mellitus for instance (Stanford and Goodyear, 2014; Aoi et al., 2013). In particular, these improvements are associated with a reduced risk of colon cancer development. Moreover, exercise is used as an adjunct 


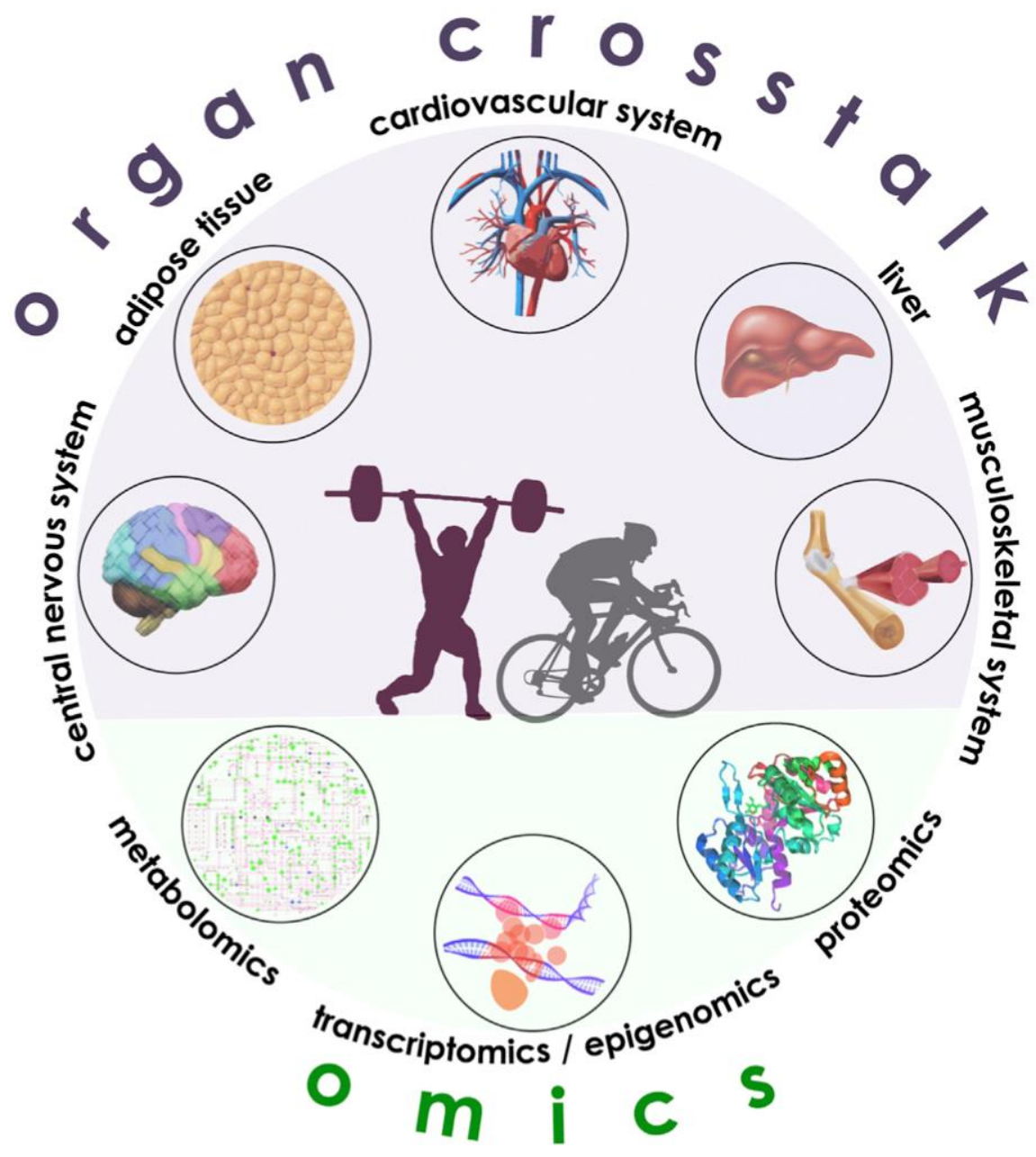

Figure II.6. An Omics approach to decipher the molecular basis of exercise adaptation. The integrated biology of the acute and adaptive response to endurance and strength exercise training requires the involvement of multiple organs to achieve physiological improvements in work performance. Future challenges will be the integration of an individual's genetic and epigenetic background, with the tissue-specific gene expression, proteome, and metabolomic profiles to predict improvements in whole-body glucose homeostasis, strength, and aerobic capacity. (from Zierath and Wallberg-Henriksson, 2015) 
in different cancer therapies (Brown at al., 2012). Cardiorespiratory fitness is another benefit of physical activity. Cardiovascular remodeling includes enhanced myocardial contractility and changes in arteries wall thickness and constrictor tone (Wilson et al., 2015). Further, physical activity was shown to positively impact different brain functions. It is associated with lower risks of cognitive impairment, Alzheimer's disease and dementia in general (Cotman and Berchtold, 2002). In addition, exercise enhances learning, contributes to maintain cognitive functions during aging and increases resistance to brain insult. Exercise-induced brain plasticity includes the promotion of neuronal survival and the stimulation of neurogenesis, the reduction of synapse hypotrophy and the development of brain vascularization (Cotman and Berchtold, 2002). Finally, specific training can ameliorate symptoms of neuromuscular diseases including myopathies and motor neuron diseases (Anziska and Sternberg, 2012). For example, appropriate physical training ameliorates motor skills and cognition, and has a positive psychological impact on Parkinson's disease patients (Petzinger et al., 2013). Exercise is particularly beneficial in slowly developing degeneration such as in spinal muscular atrophy or Charcot-Marie-Tooth, or at the early stage of ALS or Parkinson's disease (Ansizka and Sternberg, 2012).

Exercise cannot only be defined in terms of skeletal muscle contraction because it challenges the whole-body homeostasis. Every organ system is involved in coordinated response to increased muscular mechanical, metabolic and thermoregulatory demands associated with the increased workload (Hawley et al., 2014 and figure II.6). The diverse but synergistic mechanisms underlying the benefits of physical activity have started to be elucidated. Some of the exercise beneficial "whole-body" effects were shown to be mediated by the skeletal muscle itself. Indeed, upon contractile activity skeletal muscle communicates with other organs including adipose tissue, liver, pancreas, bone and brain, by the release of various peptides and cytokines (Pedersen and Febbraio, 2012). The so-called myokines exert various autocrine, paracrine, or endocrine effects. For example, interleukin-6 can modify glucose metabolism (Pedersen et al., 2003), meteorin-like induces browning of white fat (Rao et al., 2014), kynurenic acid acts on brain depressive centers (Agudelo et al., 2014) and SPARC (also known as osteonectin) is implicated in the susceptibility to colon cancer (Aoi et al., 2013). In response to myokineinduced remodeling, the target tissues may also release endocrine factors. For 
instance, upon muscle release of Irisin, subcutaneous adipose tissue adopts characteristics of beige adipocytes (Boström et al., 2012). In turn, new beige adipocytes release a systemic factor that enhances glucose uptake in oxidative skeletal muscle and brown fat tissue (Stanford et al., 2015). Although the molecular and cellular mechanisms remain incompletely described, the field of exercise science has a long history and the health-promoting benefits of exercise are now well appreciated. It has become evident that mitochondrion is a key element of exercise biology that has the potential to impact every tissue of the organism. As an example, it has been recently demonstrated that endurance exercise induces systemic mitochondrial rejuvenation that rescues progeroid symptoms in mitochondrial DNA (mtDNA) mutator mice (Safdar at al., 2011).

\subsubsection{Skeletal muscle adaptations to exercise}

Besides muscle communication with other organs, skeletal muscle fibers themselves undergo multiple changes in order to adapt their properties to the increased demand in energy and oxygen. Altered motor nerve activity patterns can cause muscle structural and functional remodeling during adult life. Cross-innervation experiments have shown that innervating an adult fast muscle with a slow nerve converts it to slow. Conversely a fast nerve transformed a slow muscle into fast (Buller et al., 1960). Later, chronic nerve stimulation experiments reproducing either fast or slow nerve frequency patterns were used to induce contraction in fast or slowtwitch muscles. Fast muscles receiving slow-like stimulation acquired slow properties and vice-versa (Pette at al., 1985; Gordon et al., 1997). The transformations included complete change in myofibrillar protein expression, sarcoplasmic reticulum function, energy metabolism or transcriptional activity. Moreover, chronic low frequency stimulation of fast muscle lead to biochemical and ultra-structural changes in muscle mitochondria, coupled with an increase in the oxidative enzyme levels (Reichmann at al., 1985).

Although artificial electrical stimulation of skeletal muscle triggers intracellular pathways with putative roles in training adaptation, voluntary exercise involving the whole body induces a variety of additional physiological responses. Exercise has been shown to induce profound muscle remodeling (Bassel-Duby and Olson, 2006; 


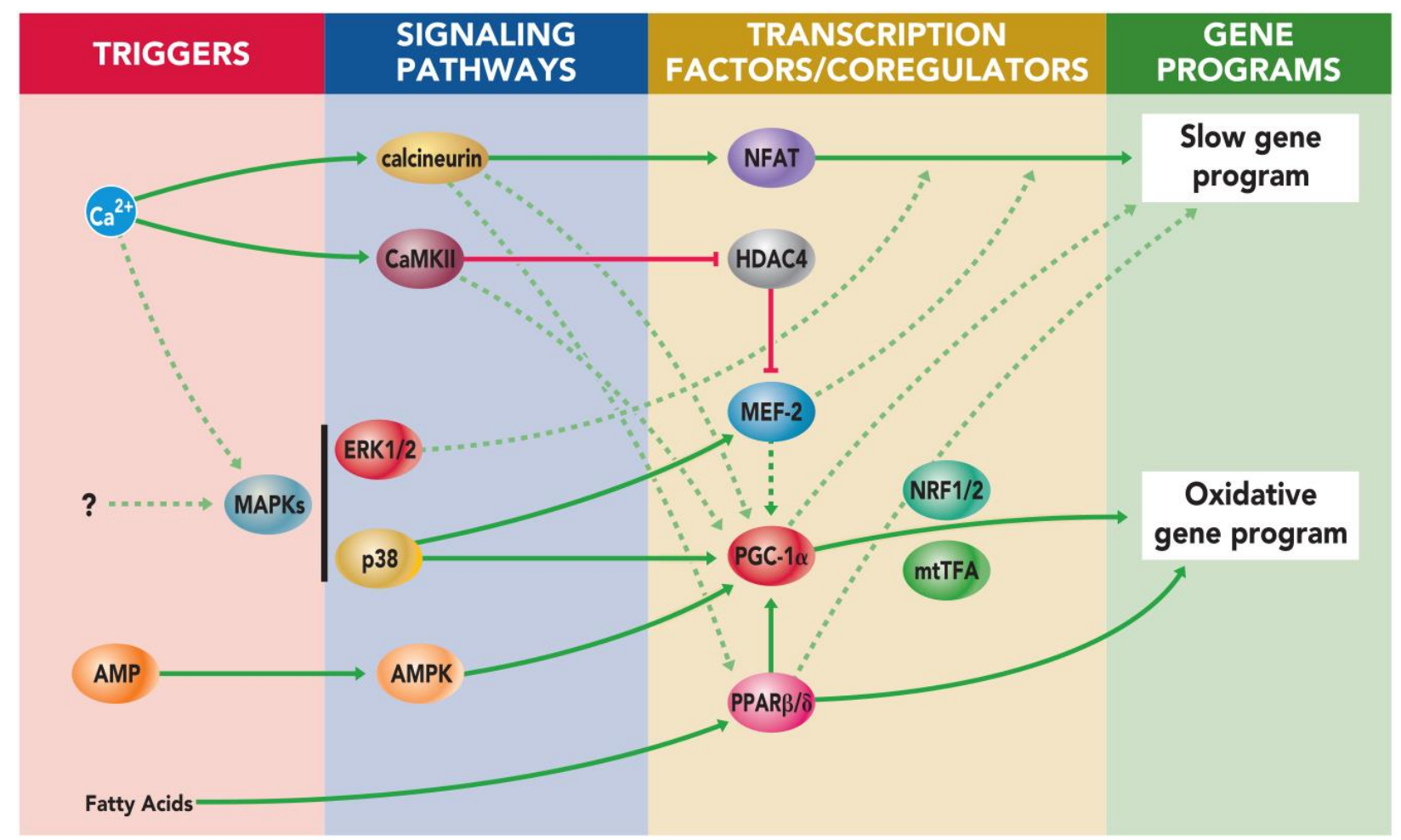

Figure II.7. Scheme of the signaling pathways and transcriptional factors and co-regulators involved in the control of slow gene program and oxidative gene program. The scheme highlights the major role of the calcineurin-NFAT pathway in the regulation of the slow gene program and the role of PPAR $\beta / y$ and PGC- $1 \alpha$ and $-1 \beta$ in the regulation of the oxidative gene program. Dotted lines indicate less established pathways. (from Schiaffino et al., 2007)

Protein functions and abbreviations. Protein phosphatase: Calcineurin; protein kinases: ERK1/2 () MAPKs, CaMKII, AMPK (AMP-activated protein kinase), p38 MAPK; transcription factors: NFAT (nuclear factor of activated $T$ cells), NRF1/2 (nuclear respiratory factors), mtTFA (mitochondrial transcription factor A), MEF2 (myocyte enhancer factor-2), PPAR $\beta / \delta$ (peroxisome proliferatoractivated receptor $\beta / \delta$ ); transcriptional co-activator $P G C-1 \alpha$ (peroxisome proliferative activated receptor- $y$ coactivator $1 \alpha$ ), other enzyme: HDAC (histone deacetylase). 
Schiaffino et al., 2007). However, the nature and the range of these modifications largely depend on both the characteristics of the training and the type of the muscle fibers. First, the frequency, intensity and duration of exercise determine the adaptive response of the muscle in terms of endurance and strength (Baar, 2009). A repetitive, low load exercise such as endurance training increases aerobic capacity, while resistance training that impose a high load on the muscle increases muscle mass and force production. Second, the exercise-induced transformations in a given fiber are limited by the intrinsic properties of this fiber (Talmadge et al., 2004). The fast-to-slow shift in muscle fiber type composition (MyHC isoforms and metabolic properties) were shown to be more pronounced in fast compared to slow muscles, which already contain a larger proportion of type I oxidative fibers. Endurance training drives the adaptation towards slow fatigue resistance fiber type. Fast muscles have the capacity to adapt in the range Ilb $\leftrightarrow$ IIx $\leftrightarrow$ Ila, while slow muscle range is $\mathrm{I} \leftrightarrow \mathrm{Ila} \leftrightarrow \mathrm{Ilx}$ (Ausoni et al., 1990)

Muscle fiber plasticity in response to increased physical activity involves multiple signaling pathways, many of which converge on each other (Bassel-Duby and Olson, 2006; Schiaffino et al., 2007). The cumulative activation and/or repression signals precisely adjust the expression of a myriad of genes involved in myogenesis, glucose metabolism, lipid metabolism, angiogenesis or transcription of mitochondrial genes. Several molecules can act as activation signal of remodeling pathways. Upon MN activity, ACh is released at the NMJ and binds to the AChRs. This in turn induces a release of calcium from the sarcoplasmic reticulum. The elevation of intracellular calcium concentration is necessary for muscle contraction but also activates calcineurin and CaMKII (calcium/calmodulin-dependent protein kinase), two enzymes involved in myosin gene expression program (Crabtree, 2009; Rose et al., 2006). Activation of calcineurin also induces expression of myoglobin, which is important in maintaining oxygen consumption and tension generation in muscle (Cole, 1982). Moreover, in response to workload, metabolic stress is increased because of ATP depletion. AMPK (AMP-activated protein kinase) senses the increase in AMP-to-ATP ratio and regulates muscle metabolic profile by initiating muscle mitochondrial biogenesis (Zong et al., 2002). Mitochondrial biogenesis is further promoted by the activation of MAPK (mitogen-activated protein kinase) pathway through yet unknown messenger(s) (Akimoto et al., 2005). Finally, increased delivery of free fatty acids 


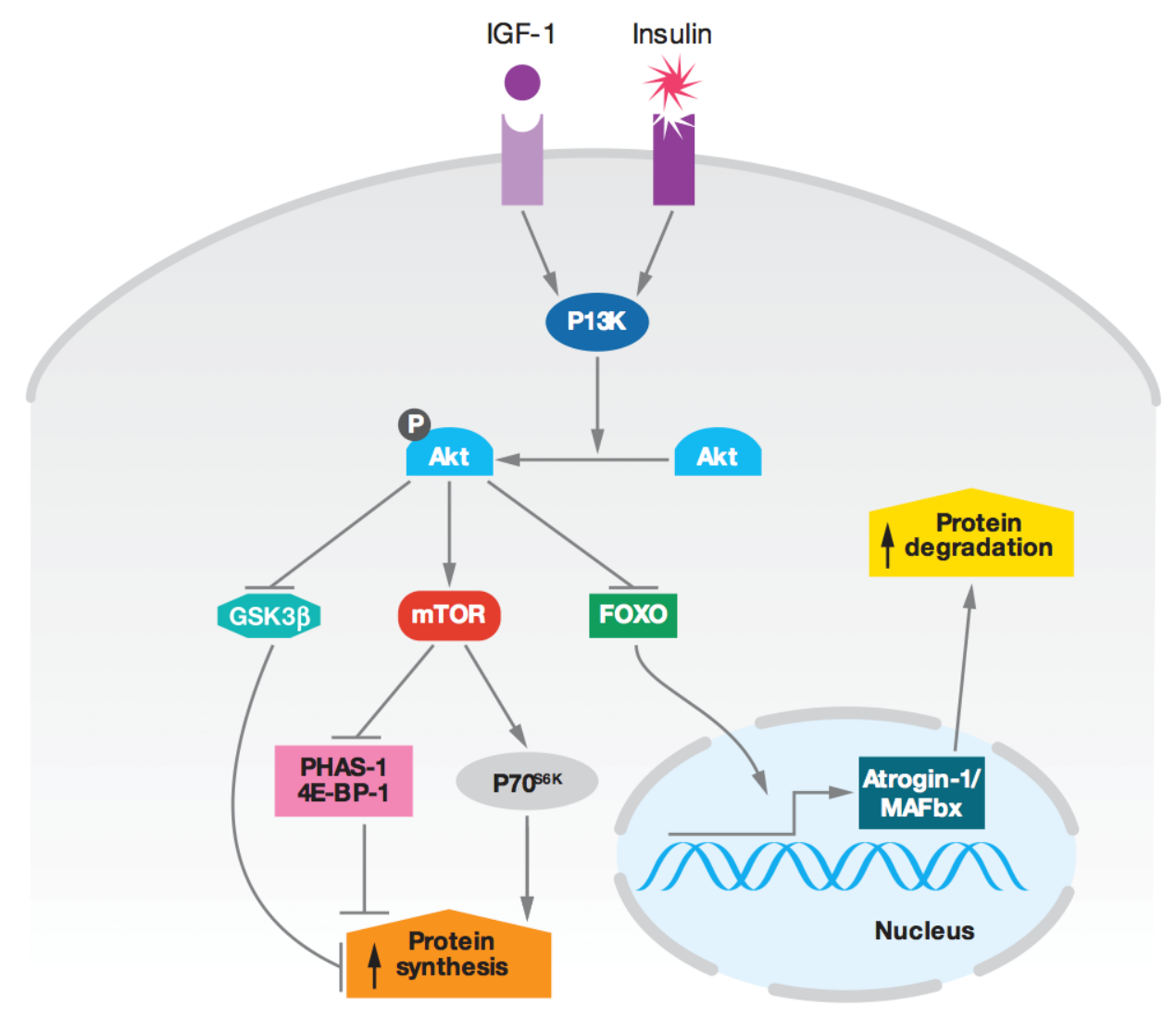

Figure II.8. Signaling pathways in hypertrophy and atrophy. In response to IGF, the Akt/mTOR signaling pathway is activated. Phosphorylated Akt phosphorylates FOXO, inhibiting FOXO nuclear entry. Activation of mTOR by Akt promotes protein synthesis and increases muscle mass, resulting in hypertrophy. In disease states, Akt is not activated, and unphosphorylated FOXO enters the nucleus and induces the atrogin-1/ MAFbx expression gene, promoting muscle atrophy. Abreviations: IGF (insulin-like growth factor), Akt/mTOR (protein kinase B/ mammalian target of rapamycin), FOXO, MAFbx (muscle atrophy F-box). (from Bassel-Duby and Olson, 2006) 
during exercise drives activation of PPARs (peroxisome proliferator activator receptors) that leads to the expression of muscle genes promoting selective utilization of lipid substrates (Muoio et al., 2002). The mechanisms underlying skeletal muscle remodeling in response to physical activity are reviewed in details in Bassel-Duby and Olson, 2006 and Schiaffino et al., 2007. These pathways induce a vast remodeling of the skeletal muscle mitochondria proteome. Many subunits of the electron transport chain, enzymes of the tricarboxylic acid cycle, phosphotransfer enzymes, and regulatory factors in mitochondrial protein synthesis, oxygen transport, and antioxidant capacity are altered after exercise training (Egan et al., 2011 and figure II.7).

Skeletal muscle can further adapt to workload by activating the insulin-like growth factor 1 (IGF-1) pathway that promotes increase in myofiber size (BasselDuby and Olson, 2006). During hypertrophy, the PI3K/AKT/mTOR (phosphatidylinositol 3-kinase/ protein kinase B/ mammalian target of rapamycin) signaling pathway is activated (Bodine et al., 2001). Direct and indirect targets of AKT include mTOR and GSK3 $\beta$ (glycogen synthase kinase 3). mTOR is a kinase, sensitive to rapamycin, whose downstream targets increase protein translation initiation and elongation, promoting protein synthesis (figure II.8). Another important remodeling signal is the regulation of glucose uptake via GLUT4 (glucose transporter 4) (Stanford and Goodyear, 2014). Both insulin and exercise increase skeletal muscle glucose uptake by the translocation of GLUT4 to the plasma membrane, but through distinct signaling mechanisms. On one hand, insulin signaling involves rapid phosphorylation of the insulin receptor and IRS-1/2 (insulin receptor substrate-1/2) on tyrosine residues, and the activation of PI3K (Goodyear et al., 1995). On the other hand, increased insulin sensitivity and responsiveness in skeletal muscle induced by contractile activity does not require tyrosine phosphorylation of molecules involved in the initial steps of insulin signaling (Goodyear et al., 1995 and figure II.9). Finally, there is increasing evidence of microRNAs implication in exercise-mediated skeletal muscle remodeling (Safdar et al., 2009).

To summarize, the possibility of adjusting the relative proportions of the fiber types present in a muscle, together with the principle of minimum-energy expenditure in motor unit recruitment, constitutes an optimal adaptation of the neuromuscular system for practically all types of muscular performances normally encountered 


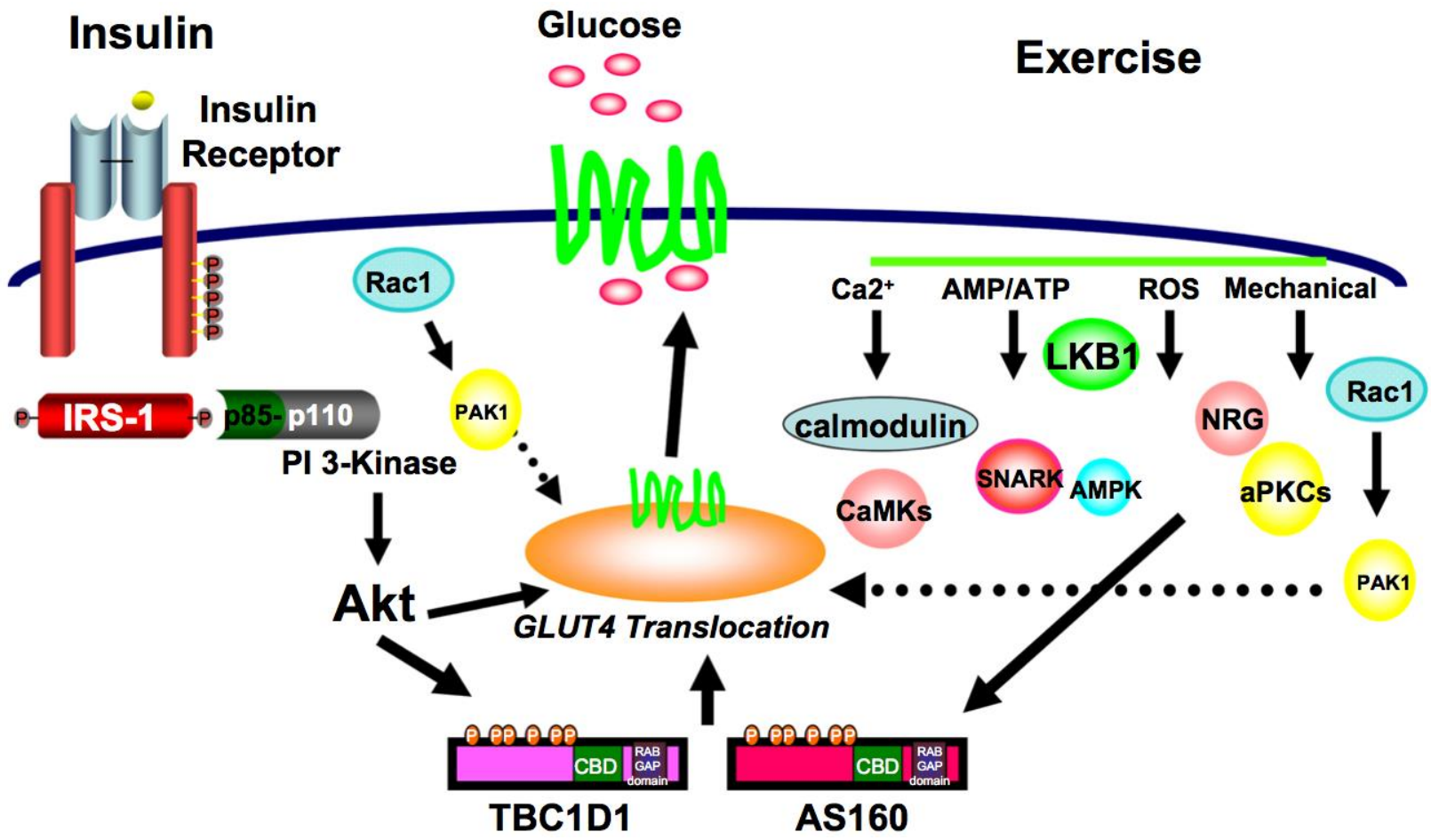

Figure II.9. Exercise and insulin regulation of glucose transport. A proposed model for the signaling pathways mediating exercise- and insulin- induced skeletal muscle glucose transport is shown. IRS-1, insulin receptor substrate-1; PAK, p21 protein (Cdc42/Rac)-activated kinase 1; LKB1, liver kinase B1; PI3K, phosphatidylinositol 3-kinase; CaMK, Ca2+/calmodulin-dependent protein kinase; SNARK, sucrose nonfermenting AMP-dependent protein kinase (AMPK)-related kinase; NRG, neuroglian; aPKCs, atypical PKCs; GLUT, glucose transporter; TBC1D1, Tre-2/USP6, BUB2, cdc16 domain family member 1; AS160, Akt substrate of $160 \mathrm{kDa}$; CBD, calmodulin-binding domain. (from Stanford and Goodyear, 2014) 
(Hatze and Buys, 1977).

\subsubsection{Modifications in spinal motor neurons upon physical training}

The motor unit has a dynamic nature (Edstrom and Grimby, 1986). As discussed earlier, skeletal muscle exhibits high degree of plasticity and exercise training elicits various fiber type transformations. Because the properties of the MNs are matched to the properties of the muscle fibers they synapse with, it is reasonable to hypothesize that $\mathrm{MN}$ undergo transformations when the muscle fibers change properties (Kernell et al., 1999). Indeed, chronic electrical stimulation of motor nerves following a slow-like pattern not only shifted muscle fiber from fast to slow phenotype, but also changed MN properties in the same direction (Munson et al., 1997). MNs become more excitable under these conditions, exhibiting decreased rheobase, increased input resistance, as well as altered after-hyperpolarization duration. However, the extent of changes observed in MNs appeared to be limited compared to the muscle fibers. In addition, although exercise-induced changes have been extensively studied, less is known about neurons and MNs adaptation to increased activity.

MNs are large and active cells, which have a high energy demand far from the cell body. Exercise has a cost in terms of energy expenditure and thus can be seen as a supplemental energetic stress for the cell. Surprisingly, studies suggest that exercise is beneficial for brain health and can be neuroprotective (Cotman and Berchtold, 2002). More interestingly, appropriate physical training in a mouse model of ALS, a neuromuscular degenerative disease, was shown to promote MN survival, which delayed the onset of motor deficits (Carreras et al., 2010; Deforges et al., 2009). The mechanisms by which exercise confers neuroprotection still remain unclear. However, a few processes have been identified that may underlie part of the observed neuroprotective effects. For example in the spinal cord, exercise was shown to induce metallothioneins (scavengers of reactive oxygen species) and limit astrogliosis in ALS (Hashimoto et al., 2009; Deforges et al., 2009).

Elucidating the mechanistic basis of neuromuscular plasticity in response to elevated muscular activity is therefore of great importance in understanding many diseases, and potentially develop new therapies. Various factors can influence the 
properties of $\mathrm{MNs}$, including neurotrophic factors, neuromodulatory inputs and exercise (Gonzalez and Collins, 1997; Heckman et al., 2009; Gardiner et al., 2006). Alike muscles, MNs underdo different changes depending on the type of exercise as well as on their intrinsic properties. Generally, voluntary running induces greater changes in slow compared to fast MNs, while forced treadmill running induces more changes in fast MNs as compared to voluntary training (Beaumont and Gardiner, 2002; Beaumont and Gardiner, 2003). Literature reports a large variability in the findings, which can be attributed to the use of different species, and different intensities and types of exercise.

The motor unit displays changes in its recruitment behavior upon physical training. Chronic moderate endurance exercise of fast muscle resulted in less variable firing rates of motor units, while force training resulted in increased recruitment thresholds and reduced firing frequencies of motor units at percentages of maximal voluntary contraction (Gardiner et al., 2006). Such an adaptation would allow more precise and accurate control of increments in muscle force. Consistent adaptations of the biophysical properties of MNs were measured with increased activity. Long-term intense endurance training resulted in hyperpolarized resting membrane potential, hyperpolarized voltage threshold, faster action potential rise time, and, in fast MNs, increased estimated capacitance (Beaumont and Gardiner, 2003). Upon voluntary wheel running, similar adaptations were found in resting membrane potential and voltage threshold, with the addition of increased afterhyperpolarization amplitude. However, these adaptations were restricted to slow MNs (Beaumont and Gardiner, 2002). Moreover, results from a chronic muscle overload experiment suggested higher excitability of fast-type MNs innervating the overloaded muscle, and a shift towards slow-type electrophysiological properties (Krutki et al., 2015). The response to the increased level of chronic activation of MNs was shown to be relatively quick and stable. Together, the studies suggest that adaptations in MNs with exercise training may involve alterations in ion conductances.

Further, MNs exhibit changes in their morphological characteristics and functional adaptations upon exercise. With increased voluntary activity, several studies have shown a slight, although statistically significant, increase in volume of the MN soma and size of the dendritic arborization. In contrast, contradictory conclusions were reported on changes in axon diameter in peripheral nerves. These 
findings generally indicate minor morphological changes in MNs as a result of increased chronic neuromuscular activity (Gardiner et al., 2006). Trained MNs also showed increase in axonal transport in both orthograde and retrograde directions (Jasmin et al., 1988). This transport is most likely important for the delivery of substances to and from the periphery. Following endurance training an increase in the concentration of the synaptic protein CGRP (calcitonin gene-related peptide) was reported in the soma and axon of MNs (Gharakhanlou et al., 1999).

Finally, MNs present evidences of metabolic/biochemical changes in response to exercise. Like in the muscle, exercise regulates expression of metabolic enzymes in the spinal cord, although changes may differ between the two tissues. In one study, the mitochondrial enzyme SDH (succinic dehydrogenase) was found to have an increased activity in slow but not fast MNs, corresponding to the different levels observed in slow versus fast muscle fibers (Nakano et al., 1997). However, another study found no difference in SDH activity after training. So it is still unclear whether exercise elicits modifications of MN mitochondrial content (Seburn et al., 1994). These findings may reflect differential adaptation of distinct types of MNs to specific types of training. Further, a brain-derived neurotrophic factor (BDNF)-mediated mechanism was reported to promote neuroplasticity in both the hind limb muscles and the lumbar spinal cord following acute voluntary exercise (Gomez-Pinilla et al., 2002). Moreover, transcriptional changes in MNs following prolonged voluntary and treadmill training were shown to be consistent with the previously reported electrophysiological results and demonstrated a transition toward MNs that innervate slower muscle fibers (Woodrow et al., 2013). Taken together, all these findings indicate that $\mathrm{MNs}$ do undergo adaptive plastic changes in response to endurance training.

Few MN markers have been identified yet and only some of them where shown to be associated with MN differential susceptibility to degeneration (MMP9, SIL1). MN subtype differential adaptation to exercise and the mechanisms underlying its potential protective effect remains to be elucidated.

\subsubsection{Changes in the neuromuscular junction induced by exercise}

Exercise induces multiple changes in muscle as well as in MNs. As a 
consequence, the neuromuscular synapse is also submitted to remodeling upon physical activity (Nishimune et al., 2014). The rate of remodeling may differ depending on the type of muscle, e.g. slow- versus fast-twitch, and the type and intensity of exercise, e.g. endurance versus resistance training (Andonian and Fahim, 1988; Waerhaug et al., 1992; Deschenes et al., 1993). Exercise was shown to impact fast and slow presynaptic terminals differently. For instance, after endurance training, the absolute nerve terminal area and length were increased in slow but not in fast muscles, whereas the density of nerve terminal varicosities was reduced in fast but not in slow muscles (Waerhaug et al., 1992). In a high intensity running paradigm, the nerve terminal area was increased in slow muscles and to a greater extend in fast muscles. Fast muscles also had enhanced nerve terminal branching and complexity (Andonian and Fahim, 1988). In addition, the rate of change in a given muscle was shown to depend on exercise intensity. For example, nerve terminal branching and complexity was increased in the NMJs of a slow muscle, but the change was more pronounced in the high-intensity compared to the low-intensity trained animals (Deschenes et al., 1993). Further, remodeling also occurs at the postsynaptic membrane of NMJs. For example, training induces hypertrophy of the post-synapse in slow muscles (Deschenes et al., 1993). This exercise-induced increase in NMJ size correlates with the hypertrophy of the fibers in slow muscles, which suggests that part of these adaptive changes of NMJs is probably a secondary effect of muscle fiber change in diameter induced by exercise (Waerhaug et al., 1992; Deschenes et al., 1993; Balice-Gordon et al., 1990).

In addition to the NMJ morphological changes, exercise was also reported to alter synaptic transmission. Endurance training improves neuromuscular transmission efficacy in slow as well as in fast muscles (Desaulniers et al., 2001; Argaw et al., 2004). The elevated safety factor of the synaptic transmission in trained muscles is due to increased transmitter release (Dorlöchter et al., 1991). In response to the presynaptic changes, AChE content and AChR number are increased in muscle fibers resulting in the strengthening of the synapse (Jasmin et al., 1987; Desaulniers at al., 1998). The molecular mechanisms that mediate exercise benefits on NMJs have not yet been fully elucidated. However, many lines of evidence indicate an increase in motoneuronal protein synthesis, axon transport, and secretion of trophic substances that influence gene transcription in muscles (Gardiner et al., 
2006). For example, SNAP25 (synaptosomal-associated protein 25), a protein important for the docking of the transmitter vesicles to the presynaptic membrane, is selectively transported in higher quantities in axons of trained MNs (Kang et al., 1995). Exercise increases the phosphorylation and proteolytic processing of the signaling molecule neuregulin-1 in skeletal muscle (Lebrasseur et al., 2003). Neuregulin signaling is known to induce expression of synaptic genes in muscles and may also play a role on the presynaptic side of NMJs (Sandrock et al., 1997; Wolpowitz et al., 2000). Physical training induces expression of a muscle-specific splice variant of IGF-1 that was shown to preserve NMJs in an ALS mouse model (Hameed et al., 2003; Dobrowolny et al., 2005). These results suggest that IGF-1 could also play a role in the beneficial effects of exercise on NMJs. Increased activity also upregulates transcription of several other neurotrophic factors, which have beneficial effects on NMJs. For instance, electrical stimulation of the sciatic nerve was shown to increase expression of neurotrophin 4 in rat skeletal muscles, which induced the sprouting of intact adult motor nerves (Funakoshi et al., 1995). Similarly, BDNF (brain-derived neurotrophic factor) and GDNF (glial cell line-derived neurotrophic factor) are upregulated by exercise in the rat soleus muscle (DupontVersteegden et al., 2004). These neurotrophic factors increase the survival of MNs (Henderson et al., 1994; Koliatsos et al., 1993). Furthermore, neurotrophins induce continuous synaptic remodeling and modulate the synaptic transmission efficiency of adult NMJs (Keller-Peck et al., 2001; Mantilla et al., 2004; Pousinha et al., 2006). Other signaling proteins are also regulated by physical training such as the scaffolding protein Homer, a postsynaptic protein that has a role in controlling the TRP (transient receptor potential) channel activity in skeletal muscles (Yuan et al., 2003). Exercise increases skeletal muscle levels of Homer, which binds directly to the transcription factor NFATc1 (nuclear factor of activated T cells, cytoplasmic, calcineurin dependent 1 ) that in turn upregulates the expression of the synaptic proteins AChE and utrophin in skeletal muscles (Salanova et al., 2011; Cohen and Randall, 2004; Angus et al., 2005; figure II.10).

On one hand, although synaptic terminals are optimized to maximize information transmission at minimum energy cost, most brain energy is used on synapses (Harris et al., 2012). On the other hand, axon terminals in general are characterized by their remarkable concentration of mitochondria (Palay, 1956). Thus, 


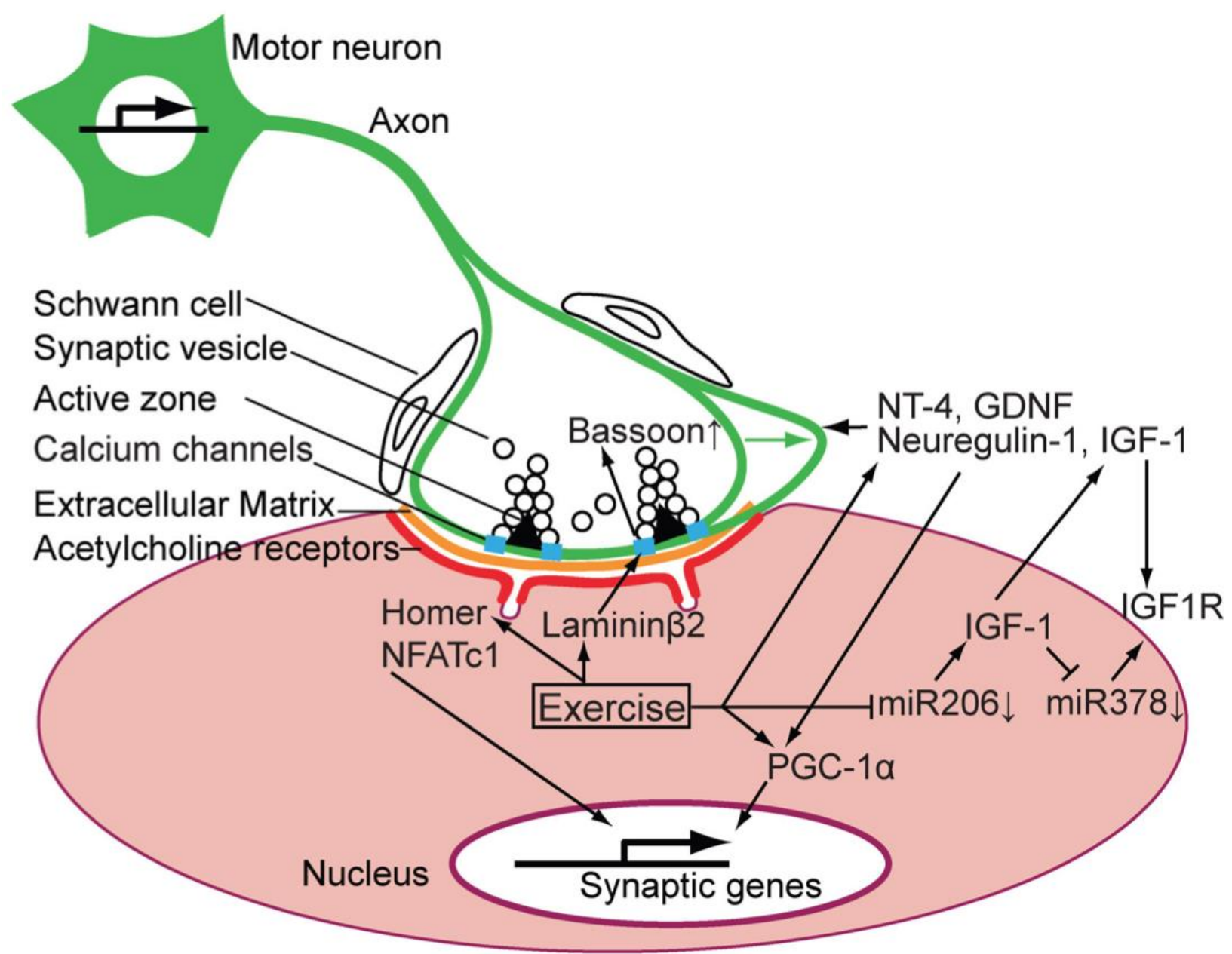

Figure II.10. Genes and proteins controlled by exercise at the vertebrate neuromuscular junction. Solid arrows represent an upregulation or enhancement of RNAs or proteins by exercise. Tshaped arrows represent a suppression of RNAs or proteins by exercise. A motor neuron and its presynaptic terminal are indicated in green. The green arrow indicates hypertrophy or sprouting of the motor nerve terminal induced by exercise. Synaptic vesicles and voltage-dependent calcium channels accumulate near the active zone indicated using a black triangle, which depicts the electron dense material of the active zones detected by electron microscopy. A muscle fiber is indicated in pink with acetylcholine receptors indicated in red and synaptic extracellular matrix indicated in orange at the synaptic cleft. A junctional fold is indicated as a trough on the postsynaptic membrane. (from Nishimune et al., 2014) 
given the large size of the neuromuscular synapses, motor endplates are likely to be the compartment of highest energy demand in the MNs. Although there is clear evidence of mitochondrial remodeling in muscles and MNs upon exercise, mitochondrial network dynamics have not yet been described at the particular site of the meuromuscular synapse in the context of increased physical activity. Thus, whether NMJ adaptations to exercise imply mitochondrial remodeling still remains to be elucidated. 


\subsection{Aim of the study}

The generation of all behavior programs in the central nervous system and their conversion into body movements is enabled by a specialized class of neurons: the motor neurons. Motor neurons control skeletal muscle contractions through direct neuromuscular synapses, the neuromuscular junctions. Thus, motor neurons are particularly active cells with a high energetic demand at locations distant from the cell body. Paradoxically, although the particularly high-energy demand of motor neurons could make them more prone to energetic stress, endurance exercise appears to be beneficial in specific neurodegenerative conditions. Moreover, tight regulation of mitochondrial biogenesis is of utmost important for meeting elevated energy demands in neurons, and thus mitochondrial plasticity may adapt motor neuron metabolic properties to increased energetic stress.

Since axon terminals are likely the compartments of highest energy demand in the motor neurons, and that mitochondria are thought to undergo the most visible changes, the present study investigated exercise-induced changes in mitochondrial network size and organization as direct readout for the adaptations of neuromuscular junctions to exercise. The use of mice expressing genetically tagged mitochondria in motor endplates allowed direct measurements from the 3D-reconstructed structures.

Further, in order to identify the mechanisms underlying the observed adaptations, RNA sequencing was carried out on muscle, as well as dorsal and ventral spinal cord samples. Changes in the transcriptome following long-term endurance training were specifically screened for expression of genes implicated in the regulation of mitochondrial function, organization and localization, as well as genes playing a role in the regulation of synaptic transmission and morphology.

The study is designed to ultimately provide insights into mechanisms modulating mitochondrial biogenesis and stress pathways and their contribution to motor neuron function and vulnerability. 


\section{Material \& Methods}

\subsection{Laboratory reagents}

The following table lists the solutions, chemicals and reagents, enzymes, antibodies and kits used in the protocols described below (sections 3.2 to 3.4 ).

Primary antibodies \& toxins

\begin{tabular}{llll}
\hline Antigen & Host & Supplier & $\#$ \\
\hline Choline acetyltransferase (ChAT) & Goat & Millipore & AB144P \\
Myosin heavy chain isoform I (MyHC I) & Mouse & DSHB * & BA-F8 \\
Myosin heavy chain isoform Ila (MyHC lla) & Mouse & DSHB * & SC-71 \\
Myosin heavy chain isoform Illb (MyHC llb) & Mouse & DSHB * & BF-F3 \\
* Developmental Studies Hybridoma Bank & & &
\end{tabular}

Secondary antibodies

\begin{tabular}{lllll}
\hline Target & Conjugate & Host & Supplier & $\#$ \\
\hline Goat lgG & Alexa Fluor 555 & Donkey & Invitrogen & A-21431 \\
Mouse IgG2b & Alexa Fluor 647 & Goat & Invitrogen & A-21242 \\
Mouse lgG1 & Alexa Fluor 488 & Goat & Invitrogen & A-21121 \\
Mouse lgM & Alexa Fluor 555 & Goat & Invitrogen & A-21426
\end{tabular}

\section{Toxins}

\begin{tabular}{lllll}
\hline Toxin & Conjugate & Host & Supplier & $\#$ \\
\hline a-Bungarotoxin & Alexa Fluor 555 & Bungarus multicinctus & Invitrogen & B35451
\end{tabular}




\begin{tabular}{|c|c|}
\hline Chemical / Reagent / Enzyme & Supplier \\
\hline Ketamine $10 \%$ & Medistar \\
\hline Xylazine $2 \%$ & Riemser \\
\hline Ethanol $99.9 \%$ & Carl Roth \\
\hline Isopropanol (2-propanol) & Carl Roth \\
\hline Chloroform (Trichloromethane) & Carl Roth \\
\hline Phenol-Chloroform-Isoamyl alcohol & Carl Roth \\
\hline Na-acetate pH 5.2 & PanReac AppliChem \\
\hline QIAzol Lysis Reagent & Qiagen \\
\hline Glycoblue (15 mg/ml) & Ambion \\
\hline 10X DNase Incubation Buffer & Ambion \\
\hline DNase I, RNase-free $(2 \mathrm{U} / \mu \mathrm{l})$ & Ambion \\
\hline RNaseOUT, Recombinant ribonuclease inhibitor $(40 \mathrm{U} / \mu \mathrm{l})$ & Invitrogen \\
\hline RNase-free water & Invitrogen \\
\hline RNase AWAY & Thermo Scientific \\
\hline PBS (10X) pH 7.2 & Gibco \\
\hline Triton $\mathrm{X}-100$ & Carl Roth \\
\hline D-Sucrose (D-Saccharose) & Carl Roth \\
\hline D-Glucose & Sigma \\
\hline Paraformaldehyde (PFA) & Carl Roth \\
\hline Bovine Serum Albumin Fraction V (BSA) & Carl Roth or Roche \\
\hline Normal goat serum & Gibco \\
\hline Normal donkey serum & Gibco \\
\hline Isopentane (2-methylbutane) & Carl Roth \\
\hline Tissue-Tek O.C.T. Compound & Sakura Finetek \\
\hline Mowiol 4-88 & Carl Roth \\
\hline
\end{tabular}




\section{Solutions}

\begin{tabular}{ll}
\hline Solution & Content \\
\hline Ethanol 75\% & RNase-free water; 75\% [v/v] Ethanol 99.9\% \\
Anesthetic solution & 1X PBS pH 7.2; 10\% [v/v] ketamine 10\%; 5\% [v/v] xylazine \\
& $2 \%$ \\
Perfusion - glucose solution & 1X PBS pH 7.2; 0.2\% [w/v] D-Glucose - $\mathrm{pH} 7.2-7.4$ \\
Perfusion - PFA fixation & 1X PBS pH 7.2; 8\% [w/v] Paraformaldehyde - pH 7.2-7.4 \\
solution & \\
Low sucrose solution & 1X PBS pH 7.2; 10\% [w/v] D-Sucrose \\
High sucrose solution & 1X PBS pH 7.2; 20\% [w/v] D-Sucrose \\
Blocking solution & 1X PBS pH 7.2; 4\% [w/v] BSA; [w/v] 0.5\% Triton-X \\
Mounting solution - Mowiol & prepared according to supplier's protocol
\end{tabular}

\subsection{Animal procedures}

All procedures were performed by experienced experimenters and according to protocols approved by the Lower Saxony State Office for Consumer Protection and Food Safety. Animals were maintained under the control of a veterinarian in the animal facilities of the Max-Planck Institute for Biophysical Chemistry and of the European Neuroscience Institute in Göttingen. Light-dark cycle was maintained as 12-12 hours, except during the training period of the C57BL/6J animals, where the light accidentally remained continuously switched on between week 7 and week 9 . The temperature of the room was regulated and the room properly ventilated. Animals had ad libitum access to food and water for the entire duration of the experiment.

\subsubsection{Mouse lines}

C57BL/6J inbred wild type males (Charles River) were used for the transcriptomic analysis. For the confocal analysis, it was taken advantage of the CreLoxP system in order to allow direct visualization of mitochondria in MNs of the mice. The conditional reporter line B6;129S-Gt(ROSA)26Sortm1(CAG-COX8A/Dendra2)Dcc/J (MGI:5318240; Jackson Laboratory stock \#018385) was used to label mitochondria. 


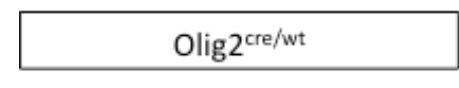

$\mathbf{X}$

Mito-Dendra2 $2^{\text {lox/lox }}$

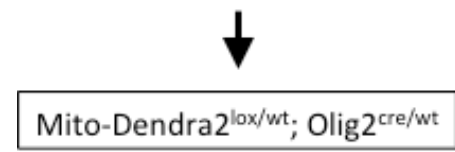

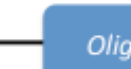

Olig 2

$\mathbf{X}$

ROSA26
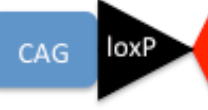

stop

MTS

Dendra2

ROSA26

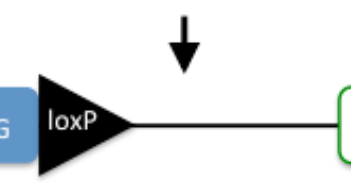

MTS

Dendra2

Figure III.1. Strategy to genetically tag mitochondria in mouse motor neurons. "Olig2-Cre" mice carry an insertion of the Cre recombinase gene expressed under the control of the Olig2 promoter. In the "Mito-Dendra2-flox" animals, the mitochondrial-targeting signal (MTS) of Cox8a is fused with Dendra2 and knocked-in to the ROSA26 locus downstream of a CAG (CMV- $\beta$ actin) promoter and a loxP-flanked Stop sequence. The Olig2-Cre mouse line is crossed with the Mito-Dendra2-flox to generate double heterozygous Mito-Dendra2lox/wt; Olig2 cre/wt. Cre-mediated selective excision of the floxed termination signal, leads to strong expression of Mito-Dendra2 in the mitochondria of Olig2 expressing motor neuron progenitors. 
In this strain, the Dendra2 fluorescent protein derived from octocoral Dendronephthya $s p$. is fused with the mitochondrial-targeting signal of subunit VIII of cytochrome c oxidase to generate "Mito-Dendra2". Mito-Dendra2 is knocked-in to the ubiquitously-expressed Gt(ROSA)26Sor gene downstream of a CAG (CMV-ßactin) promoter and a loxP-flanked Stop sequence to create a conditionally-expressed allele "Mito-Dendra2-flox" (figure III.1.). In the absence of Cre recombinase, no

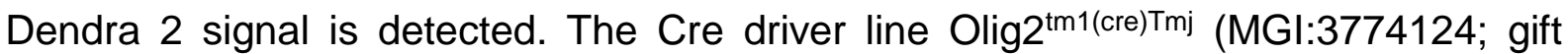
from T.M. Jessell (Dessaud et al., 2007)) was used to specifically restrict the expression of Mito-Dendra2 to the lineage of OLIG2-expressing motor neuron progenitors. "Olig2-Cre" mice carry an insertion of the Cre recombinase gene expressed under the control of the Olig2 promoter (figure III.1.). Homozygous MitoDendra2 $2^{\text {loxllox }}$ females were mated with heterozygous Olig2 ${ }^{\text {cre/wt }}$ males to give birth to double heterozygous Mito-Dendra2 ${ }^{\text {lox/wt }}$;Olig2 ${ }^{\text {cre/wt }}$ (figure III.1.). In these double heterozygotes, later referred to as "MDO" mice, the Cre-mediated recombination selectively excises the floxed termination signal, leading to strong expression of MitoDendra2 (figure III.2.). Both males and females MDO mice were used for the experiment.

The animals were genotyped using the following PCR primers:

Mito-Dendra2-flox (full protocol available on the supplier's website *) forward common 5'-CCA AAG TCG CTC TGA GTT GTT ATC-3' reverse wild type 5'-GAG CGG GAG AAA TGG ATA TG-3' reverse mutant 5'-TCA ATG GGC GGG GGT CGT T-3'

\section{Olig2-Cre}

forward 5'-TAAAGATATCTCACGTACTGACGGTG-3'

reverse 5'-TCTCTGACCAGAGTCATCCTTAGC-3'

\footnotetext{
* https://www2.jax.org/protocolsdb/f?p=116:5:0::NO:5:P5_MASTER_PROTOCOL_ID,P5_JRS_CODE:8527,018385
}

\subsubsection{Training: Voluntary wheel running}

P21 (postnatal day 21) C57BL/6J or MDO weaned animals were acclimatized to the room for one week (week -1). Each animal was housed in an individual cage for the entire duration of the experiment. At P28, mice were randomly assigned to 


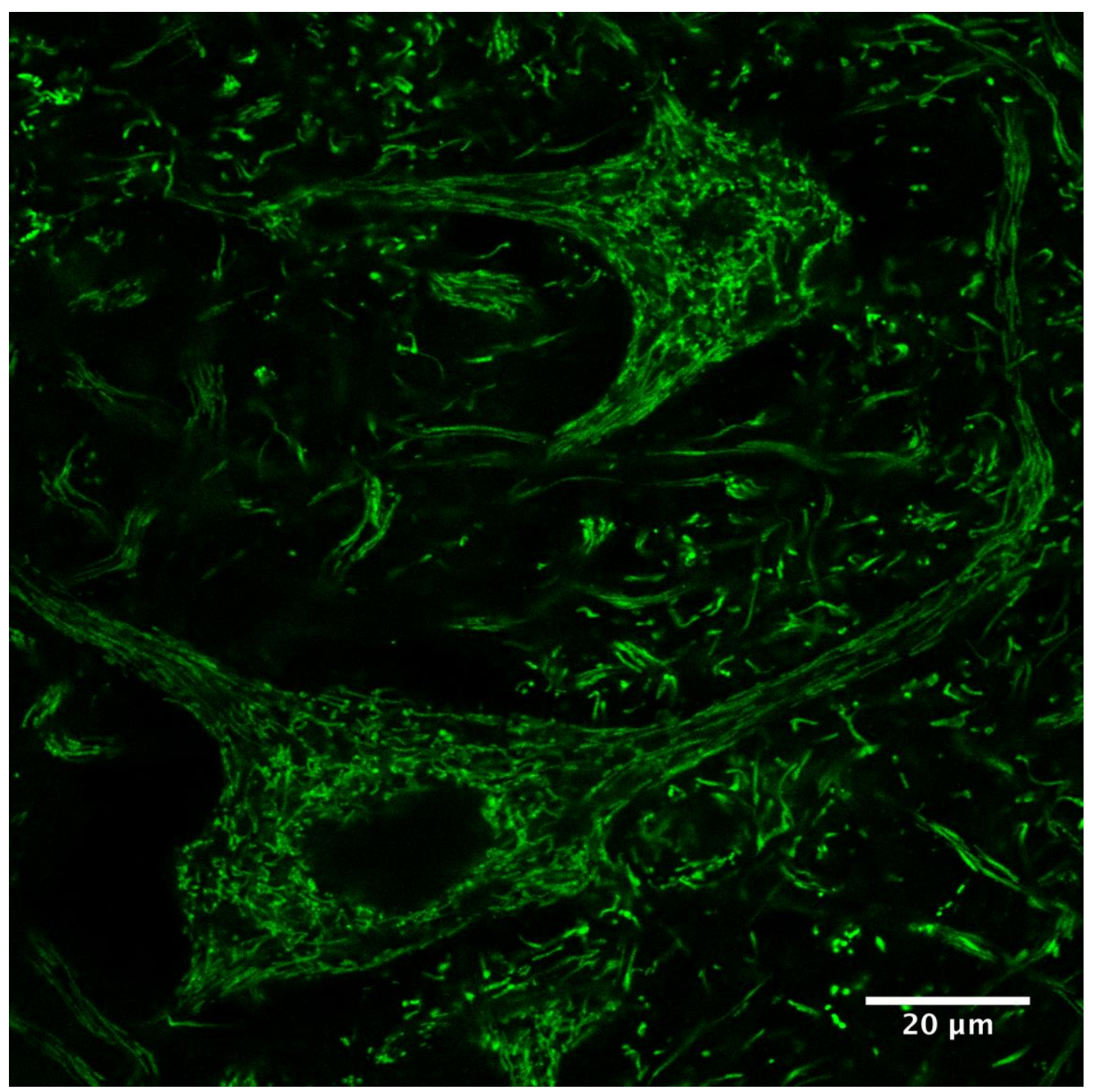

Figure III.2. Conditional expression of the Dendra2 fluorescent protein in the mitochondria of spinal motor neurons. Endogenous Dendra2 fluorescence in MNs of an adult MDO mouse (MitoDendra2 $2^{\text {lox/wt }} ;$ Olig2 $\left.2^{\text {cre/wt }}\right)$. ( $0.9 \mu \mathrm{m}$ single confocal slice of a $30 \mu \mathrm{m}$ spinal cord cryo-cross section) 
one of the following two groups: control sedentary group (ctrl) and voluntary running group (run). The two groups had comparable average body weight at the start of the training (week 0). All cages had rigorously the same size and half of them were implemented with an individual external running wheel (Panlab - Harvard Apparatus Spain). The running mice had unrestricted access to their wheel throughout the entire experiment. The mice were acclimatized to the wheel for one week (week 0 ) and the performance of each animal was recorded from P35 (week 1) for 12 weeks. The recordings consisted of the sum of complete wheel turns every $30 \mathrm{~min}, 24 \mathrm{~h} /$ day, 7days/week, for the whole training duration. The body weight of each animal was recorded at the beginning of every week. The histological analysis of NMJs was conducted on 4 sedentary and 4 trained MDO mice. Each of the ctrl and run MDO groups contained 3 females and one male. The transcriptomic analysis was conducted on 6 sedentary and 6 trained C57BL/6J males. Animals were selected based on their running performance, with a minimum cutoff set at 30000 wheel turns per week.

\subsubsection{Sample collection}

All samples were collected on week 13 after completion of training. For subsequent confocal imaging, samples from MDO animals were collected after transcardial perfusion fixation, and prepared for cryosectioning. Mice were deeply anesthetized by intraperitoneal injection of anesthetic solution $(10 \mu \mathrm{l} / \mathrm{g})$ before they were perfused with the following $37^{\circ} \mathrm{C}$ pre-warmed solutions: glucose solution for $4 \mathrm{~min}$, followed by PFA fixation solution for $8 \mathrm{~min}$. Soleus and tibialis anterior (TA) muscles were collected from the hindlimbs directly after fixation. The samples were additionally post-fixed overnight in PFA fixation solution at $4^{\circ} \mathrm{C}$. All samples were washed overnight in PBS at $4^{\circ} \mathrm{C}$ before they were incubated in low sucrose solution for $3 \mathrm{~h}$, followed by $3 \mathrm{~h}$ in high sucrose solution. Muscles were then equilibrated for $5 \mathrm{~min}$ in O.C.T. compound and placed into an embedding mold filled with O.C.T. compound. Finally the molds were snap frozen in liquid nitrogen-cooled isopentane and stored at $-80^{\circ} \mathrm{C}$ until cryosectioning.

For subsequent RNA analysis, all the following steps were carried out under RNase free conditions. C57BL/6J mice were humanely killed by cervical dislocation. 
Following dissection, the lumbar region of the spinal cord was cut into half and the ventral and dorsal regions were collected separately. The spinal samples and the soleus muscle of one hindlimb were immediately kept in $300 \mu$ QIAzol lysis reagent for subsequent RNA extraction. The soleus and plantaris muscles from the second limb were snap frozen in O.C.T. compound with liquid nitrogen-cooled isopentane. All samples were stored at $-80^{\circ} \mathrm{C}$.

\subsection{Procedures for histological analysis}

In the following procedures, "RT" refers to room temperature, comprised between 22 and $25^{\circ} \mathrm{C}$.

\subsubsection{Tissue sectioning and staining}

The O.C.T. blocks were mounted on a cryostat (CM 1510S, Leica) maintained at $-20^{\circ} \mathrm{C}$. The fixed muscle samples from MDO animals were cut into $30 \mu \mathrm{m}$ thick longitudinal sections for further high-resolution confocal imaging, while the flashfrozen muscles from C57BL/6J mice were cut into $10 \mu \mathrm{m}$ cross-sections for subsequent confocal imaging. All sections were collected on Superfrost Plus microscope slides (Thermo Scientific) and air-dried for $15 \mathrm{~min}$ before they were stored at $-20^{\circ} \mathrm{C}$.

MDO animals constitutively and selectively express the Dendra2 fluorescent protein in mitochondria of MNs and NMJs. Thus, the endogenous fluorescence in mitochondria of the pre-synapse was directly used for confocal imaging and subsequent 3D-reconstruction. The post-synapse was labeled according to the following procedure. The slides were taken out of $-20^{\circ} \mathrm{C}$ freezer and allowed to thaw for $2 \mathrm{~min}$. Then the slides were incubated with a fluorophore-conjugated $\alpha$ bungarotoxin in blocking solution for $1 \mathrm{~h}$ at $\mathrm{RT}^{\circ} \mathrm{C}$ in a humidified chamber. The slides were washed 3 times 10 min with PBS before they were mounted in Mowiol with high precision cover slips (Marienfeld-Superior, thickness No. $1.5 \mathrm{H}(0.170 \mathrm{~mm} \pm 0.005$ $\mathrm{mm})$ ).

Staining of the C57BL/6J mouse muscle followed the procedure described in 
(Bloemberg and Quadrilatero, 2012). The mouse primary and goat secondary antibodies were used as follows:

\begin{tabular}{|c|c|c|c|c|c|}
\hline & \multicolumn{2}{|c|}{ Primary antibodies } & \multicolumn{3}{|c|}{ Secondary antibodies } \\
\hline Reactivity & Antibody name & Dilution & Antibody name & Fluorophore & Dilution \\
\hline MyHC I & BA-F8 & $1: 50$ & anti-mouse lgG2b & $\begin{array}{c}\text { Alexa Fluor } \\
647\end{array}$ & $1: 500$ \\
\hline MyHC Ila & SC-71 & $1: 600$ & anti-mouse lgG1 & $\begin{array}{c}\text { Alexa Fluor } \\
488\end{array}$ & $1: 500$ \\
\hline MyHC IIb & BF-F3 & $1: 100$ & anti-mouse IgM & $\begin{array}{c}\text { Alexa Fluor } \\
555\end{array}$ & $1: 500$ \\
\hline
\end{tabular}

At the end of the staining procedure, the slides were mounted in Mowiol as described for the NMJ analysis samples.

Note: The choline acetyltransferase (ChAT) staining on figure IV.12B was obtained from a paraffin-embedded spinal cord cross-section of a C57BL/6J mouse. It was stained using a goat anti-ChAT polyclonal primary antibody at the dilution 1:200, followed by an Alexa Fluor 555-conjugated donkey anti-goat IgG secondary antibody at the dilution 1:1000.

\subsubsection{Imaging, 3-D reconstruction and statistical analysis}

The soleus is a small predominantly slow fiber type-containing muscle, mainly constituted by type I and type lla fibers. In contrast, the tibialis anterior (TA) is a large predominantly fast fiber-type containing muscle, mainly constituted of type Ilb, but also IIx and Ila fibers. To account for the size and heterogeneity in fiber type (and hence in NMJs), 10 NMJs of the soleus muscle and 15 NMJs of the TA muscle were analyzed for each animal. High-resolution image stacks of the NMJs were acquired with a laser scanning confocal microscope (LSM 710, Zeiss). All image stacks were acquired according to the Nyquist criterion for optimal resolution, using a PlanApochromat 63x/1.40 Oil DIC M27 objective (Zeiss). A maximum cutoff value for the gain in the Dendra2 channel was set in order to ensure high quality images for subsequent 3-D reconstruction. NMJs presenting too low expression of Dendra2 were not acquired. 
The 3D reconstruction of the entire synaptic structures was performed with Imaris 7 software (Bitplane). Surface objects were created to visualize the 3D structures of the pre-and post-synaptic compartments. The Imaris Filament Tracer module was used to measure the branch length of the post-synapse. All measurements were automatically exported as spreadsheets from the Imaris MeasurementPro module. Statistical comparison of soleus and TA muscle NMJs in control situation and after 12 weeks of voluntary wheel running was performed using a 2-way ANOVA followed by post-hoc pair-wise comparisons using Tukey's honest significance test for multiple comparisons. The groups compared are "soleus ctrl" with $n=40$ NMJs, "TA ctrl" with $n=60$ NMJs, "soleus run" with $n=40$ NMJs and "TA run" with $n=60$ NMJs. The test results with a $p$-value $<0.05$ were considered statistically significant. For each group, the sample distribution is represented as a dot plot. The overlaid box plot displays the median as a horizontal bar and the mean as a diamond shape. The notch shows the $95 \%$ confidence interval for the median for each group. The $\mathrm{R}$ software was used for the statistical calculations as well as for plotting ( $R$ Core Team (2015). R: A language and environment for statistical computing. R Foundation for Statistical Computing, Vienna, Austria).

The C57BL/6J muscles were imaged on a single layer with a laser scanning confocal microscope (LSM 710, Zeiss). Fiber type quantification of the cross sections was performed with Fiji (Schindelin et al., 2012) using the Cell Counter plugin (Kurt De Vos). Only three MyHC types could be stained at the same time, and therefore only the MyHC type I, Ila and Ilb described in section 3.3.1 were labeled in the experiment. MyHC type IIx fibers remained unlabeled. I-lla hybrid fibers could be reliably counted as double positive fibers (here grey and green). However, Ila-IIx (green and black) and IIx-Ilb (black and red) hybrid fibers could only be identified by a variation in the intensity of either Ila or Ilb staining. Therefore, Ila-Ilx and IIx-Ilb hybrids were counted as Ila and Illb positive fibers respectively. As a consequence, the IIx fiber count only represents the pure unlabeled IIx fibers. Student's T-tests were performed on each of the 5 fiber types to compare MyHC isoform expression between control and running animals. The test results with a $p$-value $<0.05$ were considered statistically significant. 


\subsection{Procedures for transcriptomic analysis}

In the following procedures, "RT" refers to room temperature, comprised between 22 and $25^{\circ} \mathrm{C}$.

\subsubsection{Tissue preparation - RNA isolation}

All the following steps were conducted under a fume hood in RNase free conditions. To this end, surfaces and instruments were decontaminated using RNase AWAY. Samples were taken out of the $-80^{\circ} \mathrm{C}$ freezer and briefly thawed. 10 to 15 ceramic beads of $1.4-1.6 \mathrm{~mm}$ were added to each tube. Samples were homogenized using an Omni Bead Ruptor (Omni International) as follows: soleus muscle at the speed 6.60 for 30 s and ventral and dorsal spinal cord at the speed 3.40 for $15 \mathrm{~s}$. $700 \mu l$ of QIAzol lysis reagent was added to the samples, which were then incubated $5 \mathrm{~min}$ at RT. Subsequently, $200 \mu$ l chloroform was add to the tubes before vigorous shaking for $15 \mathrm{~s}$ and incubation at RT for $5 \mathrm{~min}$. Samples were centrifuged at $12000 \mathrm{~g}$ at $4^{\circ} \mathrm{C}$ for $15 \mathrm{~min}$. Aqueous phase was transferred into a fresh tube where $500 \mu \mathrm{l}$ isopropanol and $1.5 \mu \mathrm{l}$ of Glycoblue. Samples were incubated at $-20^{\circ} \mathrm{C}$ for at least $30 \mathrm{~min}$, then centrifuged at $12000 \mathrm{~g}$ at $4^{\circ} \mathrm{C}$ for $30 \mathrm{~min}$. The supernatant was discarded and the RNA pellet washed with $1 \mathrm{ml}$ of chilled $75 \%$ ethanol before a new centrifugation at $12000 \mathrm{~g}$ at $4^{\circ} \mathrm{C}$ for $5 \mathrm{~min}$. The supernatant was discarded before proceeding with DNase treatment. $5 \mu$ l 10X Incubation Buffer, $1 \mu$ I DNase I, $0.5 \mu \mathrm{l}$ RNaseOUT and 43.5 $\mu$ R Rase-free water were subsequently added to the pellet. Samples were incubated on a thermomixer (Eppendorf) at $37^{\circ} \mathrm{C}$ for $20 \mathrm{~min}$ at $550 \mathrm{rpm}$. $150 \mu l$ RNase-free water was added followed by $200 \mu l$ Phenol-Chloroform-Isoamyl alcohol. Tubes were vortexed and centrifuged at $16000 \mathrm{~g}$ at RT for $2 \mathrm{~min}$. Upper phase was collected in a new tube where $20 \mu \mathrm{l} 3 \mathrm{M} \mathrm{Na}$-acetate $\mathrm{pH} 5.2,200 \mu \mathrm{l}$ chilled isopropanol and $1 \mu \mathrm{l}$ Glycoblue were added. Tubes were vortexed and spun before $30 \mathrm{~min}$ incubation at $-20^{\circ} \mathrm{C}$. Samples were centrifuged at $16000 \mathrm{~g}$ at $4^{\circ} \mathrm{C}$ for $30 \mathrm{~min}$. The supernatant was removed, while the pellet was washed with $1 \mathrm{ml}$ of chilled $75 \%$ ethanol and centrifuged at $16000 \mathrm{~g}$ at $4^{\circ} \mathrm{C}$ for $5 \mathrm{~min}$. The washing step was repeated once. After removal of the supernatant, the pellet was air-dried at RT. Finally, the RNA pellet was dissolved in $50-100 \mu$ RNase free water and stored at $-80^{\circ} \mathrm{C}$. 


\subsubsection{RNA sequencing and data analysis}

RNA sequencing was performed in collaboration with the laboratory of Pr. André Fischer, Epigenetics in Neurodegenerative Diseases, German Center for Neurodegenerative Diseases (DZNE) and the DZNE Next Generation Sequencing Facility, Göttingen, Germany. Prior to sequencing, the quantity of purified RNA in each sample was measured using the NanoDrop 2000 spectrophotometer (Thermo Scientific), and further quality-controlled using the 2100 Bioanalyzer system (Agilent Technologies). All the 12 samples for each tissue - soleus muscle, dorsal spinal cord and ventral spinal cord - showed RNA integrity numbers above 8.50. Library preparation and cluster generation for RNA sequencing was performed according to Illumina standard protocols (TruSeq technology, Illumina). Libraries were qualitycontrolled and quantified using the NanoDrop 2000 spectrophotometer (Thermo Scientific), the 2100 Bioanalyzer system (Agilent Technologies) and the Qubit fluorometer (Invitrogen). Base calling from raw images and file conversion to FastQ files was achieved by Illumina pipeline scripts.

The subsequent biostatistical analysis was performed in collaboration with the laboratory of Dr. Stefan Bonn, Computational Analysis of Biological Networks, German Center for Neurodegenerative Diseases (DZNE) and the DZNE Next Generation Sequencing Facility, Göttingen, Germany. The analysis included the following steps: quality control using FastQC (http://www.bioinformatics.babraham.ac.uk/projects/fastqc/), mapping to reference genome using STAR aligner v2.4.1 (Dobin et al., 2013) and read counting on genes using HTSeq v0.6.1 (Anders et al., 2015). Finally, the differential gene usage was analyzed in pairwise comparisons using the DESeq2 v1.8.0 R-package (Love et al., 2014; R Core Team (2015). R: A language and environment for statistical computing. R Foundation for Statistical Computing, Vienna, Austria). The change in expression between two conditions for each gene entry is defined by an adjusted $p$-value representing the statistical significance of the change, and a log2(fold-change) value (amplitude of the change for up- or down-regulated genes).

Gene set overlaps were calculated using Venny (http://bioinfogp.cnb.csic.es/tools/venny/). 
A functional annotation of gene products was carried out in R using the FUNC package (Prüfer et al, 2007). This ranking method allows identifying significant associations between gene sets and ontological annotations such as provided by the Gene Ontology Consortium (Ashburner et al., 2000). The ranking is based on a $\mathrm{Pi}$ value calculated for each gene: $\mathrm{Pi}=\log 2$ (fold-change) $\times(-\log 10(\mathrm{p}$-value $)$ ). A refinement algorithm enabled to identify the Gene Ontology categories enriched in the experimental samples compared to the control. The subsequent interpretation of the data was based on the Gene Ontology categories presenting a $p$-value after refinement $<0.05$. 


\section{Results}

Physical activity increases energy expenditure of the body and elevates energetic stress across cell types and organs. The neuromuscular system has particularly high energy requirements and physical activity increases muscle contraction and motor neuron (MN) firing. Since tight regulation of mitochondrial biogenesis is of utmost importance for meeting elevated energy demands - and conversely dysregulation of mitochondrial biogenesis can cause cellular demise mitochondrial remodeling was investigated in the mouse neuromuscular system (MNs and muscles) in response to increased energetic stress caused by exercise. To achieve this, mice were trained for long-term voluntary endurance running. Specific and common adaptive mechanisms were studied in terms of modifications of the mitochondrial web organization in the neuromuscular junction (NMJ), the interface between muscles and MNs. Then, differential gene expression levels were assessed in order to identify molecular changes that accompany or underpin muscle and spinal cord adaptations to exercise.

\subsection{Endurance training in wild type mice}

Two lines of wild-type animals were submitted to 12 weeks of voluntary wheel running. A group of six C57BL/6J male running mice was selected for transcriptomic analysis (fig. IV.1A, C and E), and a group of four MDO (Mito-Dendra2lox/wt; Olig2 ${ }^{\text {cre/wt) }}$ running mice were selected for histological analysis (fig. IV.1B, D and F). During the training, the C57BL/6J animals were accidentally exposed to continuous light for a brief period, which hampered the running performances of the mice. However, after return to a 12-12 light-dark cycle, mice recovered a level of activity close to that expected at this point of the training, and thus experiment was continued. This period was excluded from the evaluation of the running performances.

In the C57BL/6J group, the average daily running distance ranged from 2.1 up to $3.6 \mathrm{~km} /$ day (fig. IV.1A). The individual maximum running distance reached 4.5 $\mathrm{km} /$ day, while the lowest performance was $1.4 \mathrm{~km} /$ day. In the MDO group, the average daily running distance ranged from 3.1 to $4.7 \mathrm{~km} /$ day (fig. IV.1B). The best 

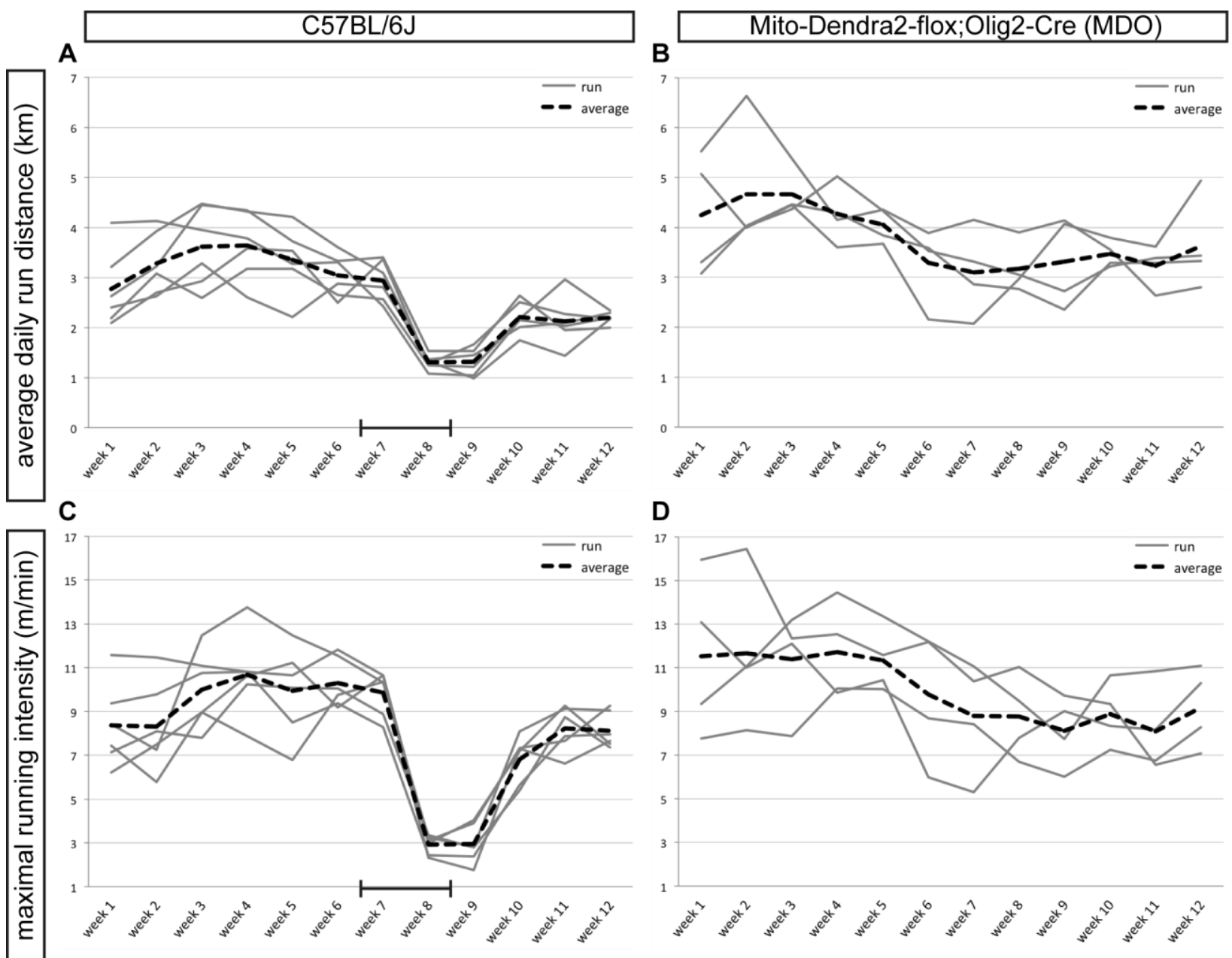

D
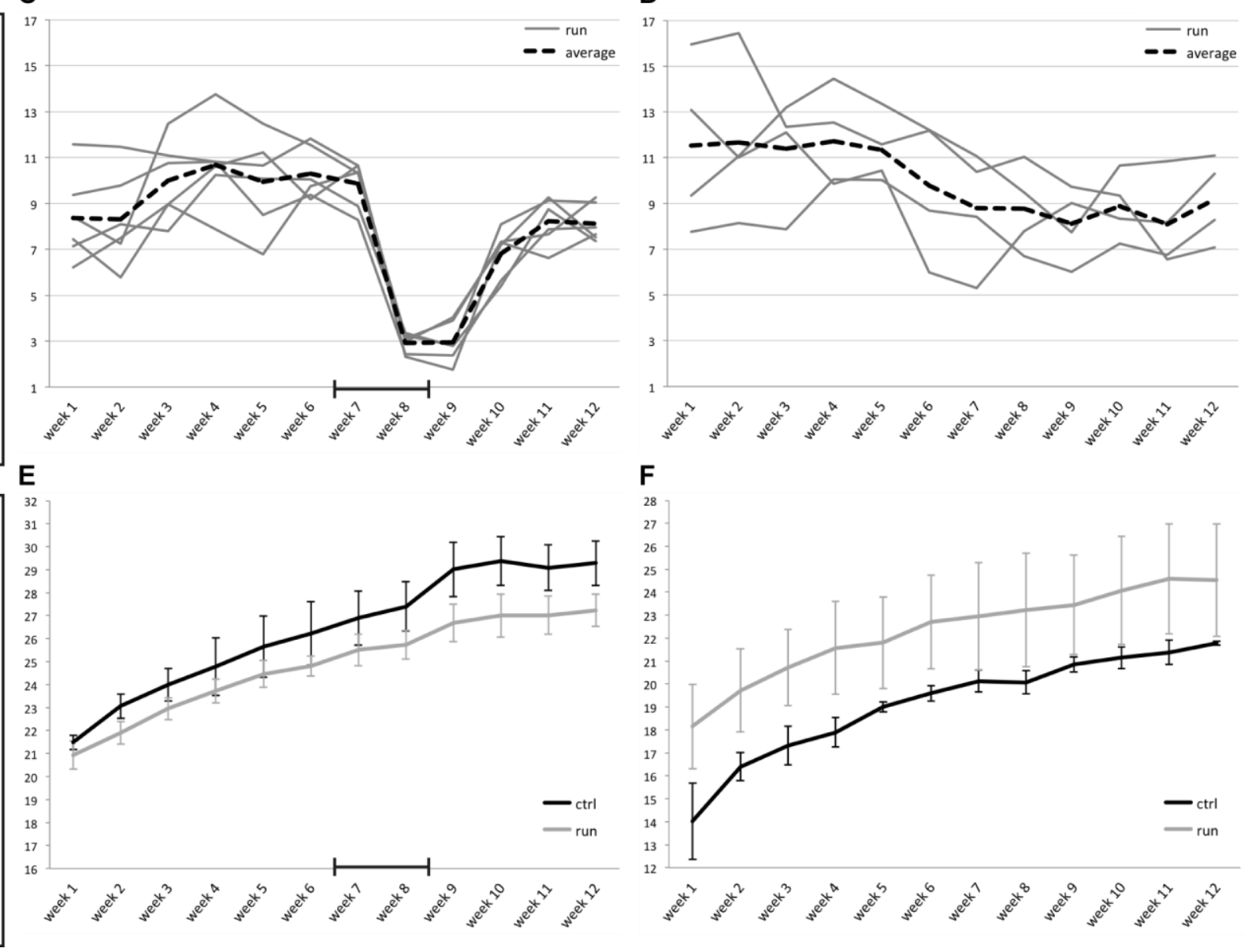

Figure IV.1. Running performances among the animals. A group of six $C 57 \mathrm{BL} / 6 \mathrm{~J}$ running mice $(A$, $C$ and $E$ ) and a group of four MDO running mice (B, D and F) were selected for transcriptomic and histologic analysis respectively. A-B. The average distance run daily slowly declined over the course of the experiment in both mouse lines. C-D. The average intensity of running during the one hour of the mouse maximal daily activity also slowly decreased over the 12 weeks of voluntary wheel running. $\mathrm{E}-\mathrm{F}$. The body weight increased in both mouse lines along the training duration and the gain in weight was similar between the control and the running groups (C57BL/6J $n=6$; MDO $n=4$; mean $\pm S D$ ). [Between week 7 and 9: 2 weeks of accidental constant light in the animal facility hampered the running performance of mice.] 
individual performance was a maximum of $6.6 \mathrm{~km} /$ day, while the shortest running distance was $2.1 \mathrm{~km} /$ day. The mice were mainly active during the dark period of the day with a peak of running within the six hours following the light switch-off. The maximal running intensity of the mice was recorded over one of these six hours (fig. IV.1C and D). The intensity of running over the 12 weeks of voluntary wheel running ranged from 8.1 to $10.7 \mathrm{~m} / \mathrm{min}$ in the C57BL/6J group, and from 8.1 to $11.7 \mathrm{~m} / \mathrm{min}$ in the MDO group. Although absolute values for the two groups cannot be directly compared because of genotype and gender differences, the MDO mice performed generally better compared to the C57BL/6J animals. However, the running distances and velocities displayed a similar slope in C57BL/6J and MDO mice. In both mouse lines the average daily running distance, as well as the maximal running intensity, were maximal at the beginning of the training and slowly declined over the course of the experiment. The body weight of trained as well as sedentary animals was recorded weekly and increased in both mouse lines along the duration of the training (fig. IV.1E and F). The parallel curves indicate that the gain in weight was similar between the control and the running groups.

\subsection{Chronic training induced a shift towards a slower type in slow and fast-intermediate muscles}

In skeletal muscles, endurance training is known to induce a shift towards properties characteristic for slow fiber types (Bassel-Duby and Olson, 2006;

Schiaffino et al., 2007). In order to assess the impact of the 12-week voluntary running exercise on muscles, fiber type characterization of the soleus and plantaris muscles was performed on four out of the six running C57BL/6J mice and corresponding sedentary control animals selected for the transcriptomic analysis. Both soleus and plantaris are shank muscles, whose contraction leads to the extension of the foot. At the same time, the soleus is a predominantly slow fibercontaining and anti-gravity muscle, while the plantaris is enriched in fast and intermediate muscle fiber types.

Exercise-induced changes in fiber type in slow and fast muscles were investigated using immunolabeling of the myosin heavy chain ( $\mathrm{MyHC}$ ) isoforms predominantly expressed in each individual muscle fiber of soleus and plantaris muscles. MyHC type I, Ila and Ilb fibers were labeled, while type IIx fibers were left 


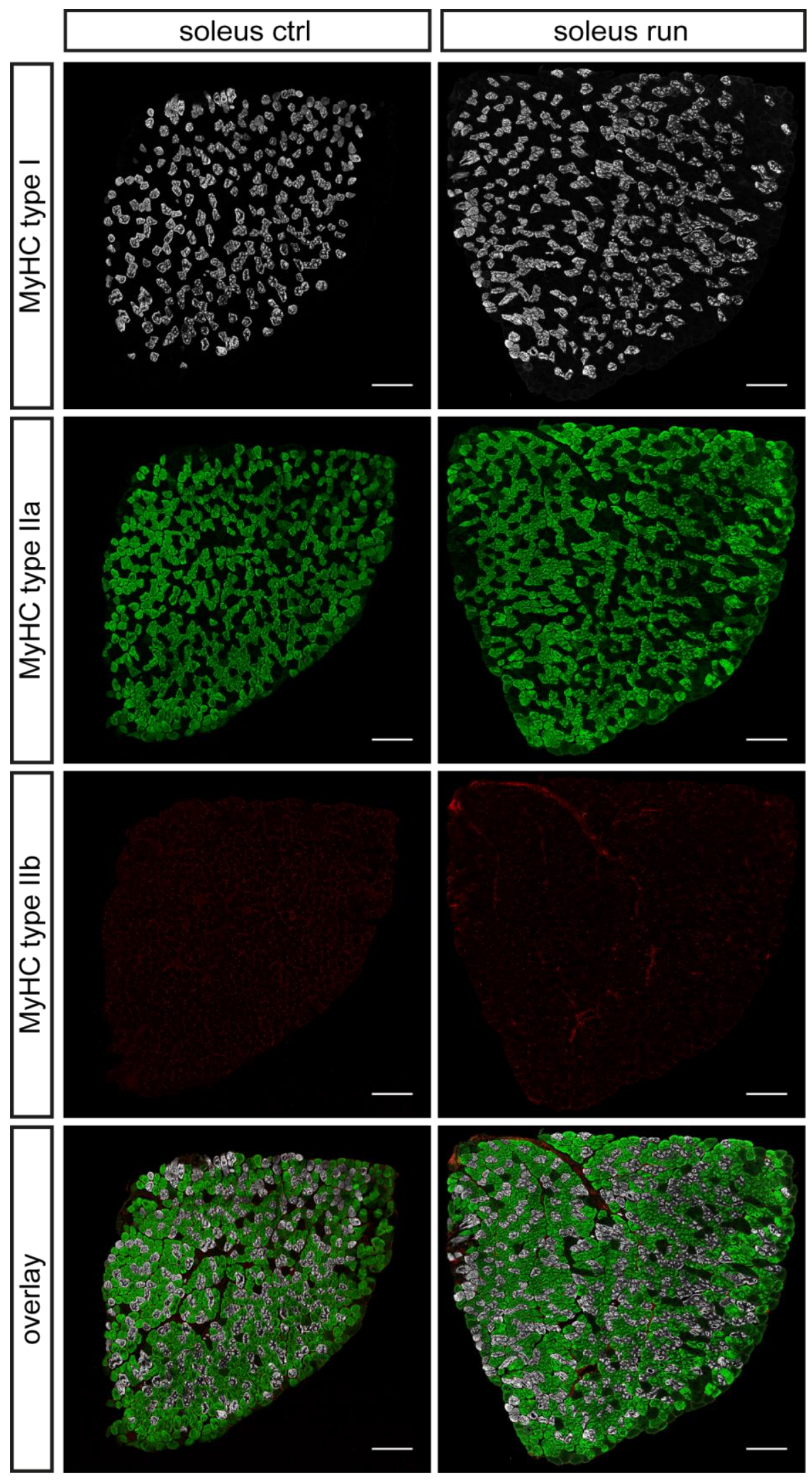

Figure IV.2. Fiber type distribution in the predominantly slow soleus muscle of sedentary and trained mice. Immunolabeling of the myosin heavy chain $(\mathrm{MyHC})$ isoforms I, Ila and Ilb predominantly expressed in soleus muscle fibers. Note: the unlabeled MyHC type IIx fibers remain black on the overlay picture. (Representative images of $n=4$ C57BL/6J mice for each paradigm; scale bars: $250 \mu \mathrm{m}$ ) 
unlabeled (fig. IV.2 and IV.3). I-Ila hybrid fibers could be reliably counted as double positive fibers (here grey and green). However, Ila-IIx (green and black) and IIx-IIb (black and red) hybrid fibers could only be identified by a variation in the intensity of either Ila or Ilb staining. Therefore, Ila-IIx and IIx-Ilb hybrids were counted as Ila and Ilb positive fibers respectively. As a consequence the IIx fiber count only represents the pure unlabeled IIx fibers. Soleus muscle fibers were only positively labeled for $\mathrm{MyHC} \mathrm{I} \mathrm{and} \mathrm{Ila,} \mathrm{while} \mathrm{showing} \mathrm{a} \mathrm{few} \mathrm{fluorescent-negative} \mathrm{fibers} \mathrm{likely} \mathrm{expressing}$ MyHC IIx (fig. IV.2 and table IV.1). In contrast, plantaris was enriched in fast fiber types Ila, Ilx and Ilb (fig. IV.3 and table IV.1).

The quantification of fiber type distribution following long-term endurance training revealed a small, but significant shift towards slower MyHC types in soleus, as well as in plantaris in the running mice (table IV.1). After training, the soleus displayed an increase in slow type I and a reciprocal decrease in fast types Ila and IIx fibers (table IV.1). However, the increased proportion of hybrid I-Ila fibers in running animals was the only statistically significant change. Similarly in the plantaris, a decrease in Ilb fibers in favor of Ilx fibers was observed. No obvious change in the number of Ila fibers was noticed. However, none of these trends were statistically significant.

Table IV.1. Muscle fiber type distribution in trained and sedentary C57BL/6J mice.

\begin{tabular}{|c|c|c|c|c|c|}
\hline Soleus fiber type & $\operatorname{ctrl} \%$ & $\pm S D$ & run $\%$ & $\pm \mathrm{SD}$ & $\mathrm{p}$-value \\
\hline I & 28.96 & \pm 1.14 & 34.26 & \pm 5.19 & 0.093 \\
\hline I-Ila & 0.74 & \pm 0.46 & 2.12 & \pm 0.96 & 0.041 \\
\hline Ila and Ila-IIx & 66.16 & \pm 3.18 & 60.82 & \pm 5.92 & 0.163 \\
\hline IIx & 4.11 & \pm 4.10 & 2.80 & \pm 3.29 & 0.637 \\
\hline IIb and IIx-IIb & 0.03 & \pm 0.05 & 0 & & 0.213 \\
\hline Plantaris fiber type & $\mathrm{ctrl} \%$ & $\pm S D$ & run $\%$ & $\pm \mathrm{SD}$ & $\mathrm{p}$-value \\
\hline I & 0.49 & \pm 0.56 & 0.10 & \pm 0.15 & 0.235 \\
\hline I-Ila & 0 & & 0 & & - \\
\hline Ila and IIa-IIx & 35.79 & \pm 6.69 & 37.62 & \pm 5.74 & 0.692 \\
\hline IIx & 15.66 & \pm 4.00 & 23.64 & \pm 6.72 & 0.088 \\
\hline IIb and IIx-Ilb & 48.06 & \pm 9.88 & 38.64 & \pm 12.46 & 0.281 \\
\hline
\end{tabular}

(two-tailed unpaired Student's T-test with $\mathrm{Cl}=0.95 ; \mathrm{n}=4 \mathrm{C} 57 \mathrm{BL} / 6 \mathrm{~J}$ mice in each group) 


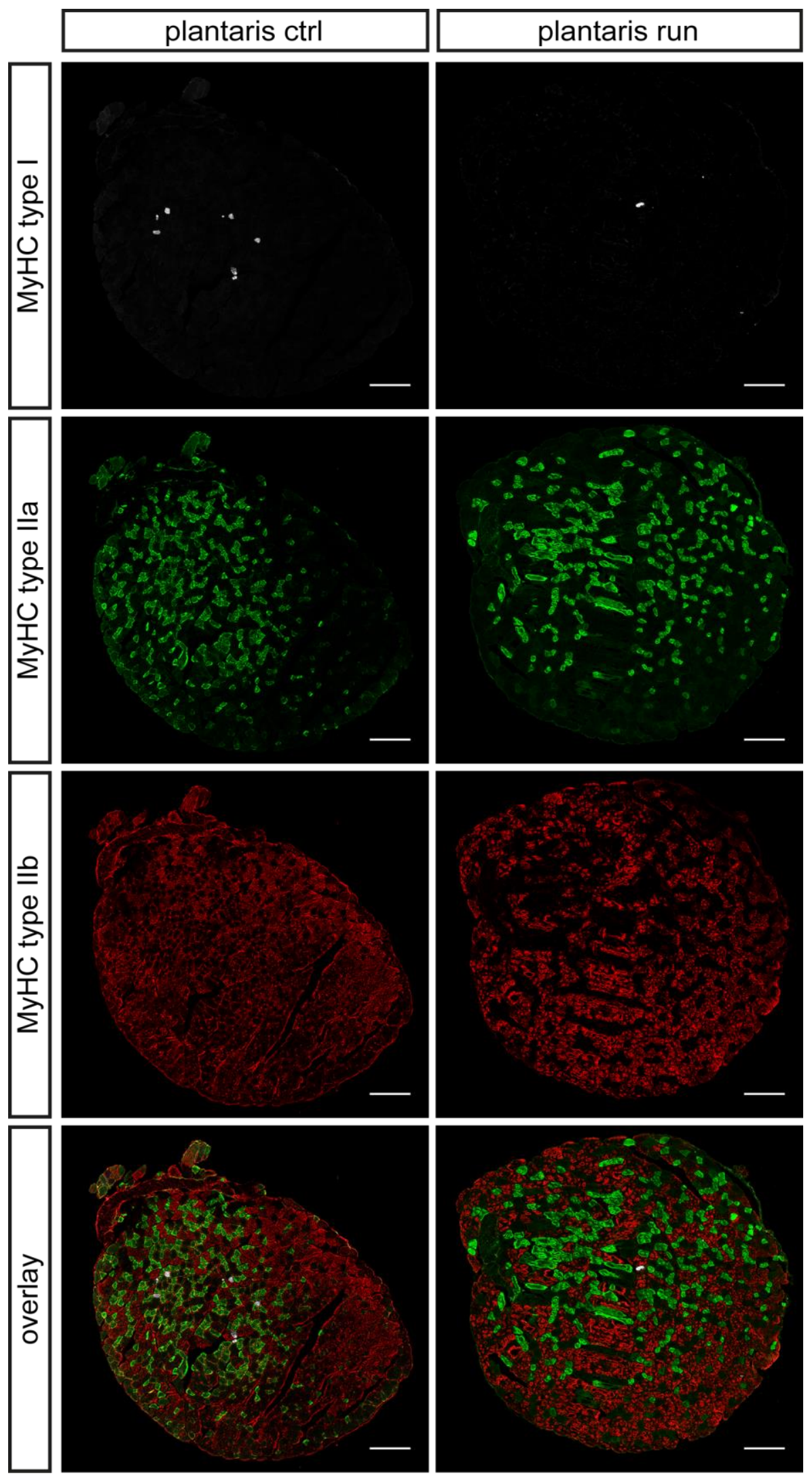

Figure IV.3. Fiber type distribution in the fast-intermediate plantaris muscle of sedentary and trained mice. Immunolabeling of the myosin heavy chain $(\mathrm{MyHC})$ isoforms I, Ila and Ilb predominantly expressed in plantaris muscle fibers. Note: the unlabeled MyHC type IIx fibers remain black on the overlay picture. Some muscle spindles expressing MyHC I are labeled on these sections. (Representative images of $n=4$ C57BL/6J mice for each paradigm; scale bars: $250 \mu \mathrm{m}$ ) 
The total number of fibers in both soleus and plantaris remained unchanged after the 12-week training period. Although there was a trend in favor of a larger total number of fibers in both soleus $(+6.4 \%)$ and plantaris $(+1.2 \%)$ muscles in trained mice compared to control, the difference was not statistically significant (table IV.2).

Table IV.2. Total number of fibers in muscles of trained and sedentary C57BL/6J mice

\begin{tabular}{lrlrll}
\hline fiber count & ctrl mean & \pm SD & run mean & \pm SD & p-value \\
\hline Soleus & 968.34 & \pm 50.44 & 1030.75 & \pm 57.33 & 0.153 \\
Plantaris & 823.42 & \pm 158.75 & 832.92 & \pm 31.29 & 0.910 \\
\hline
\end{tabular}

(two-tailed unpaired Student's T-test with $\mathrm{Cl}=0.95 ; \mathrm{n}=4 \mathrm{C} 57 \mathrm{BL} / 6 \mathrm{~J}$ mice in each group)

Together, these results suggest that the long but of moderate intensity training induces a slight shift towards slower fiber types in slow as well as in fast muscles. However, the rate of change appeared to be higher in soleus compared to plantaris, which is likely due to the preferential recruitment and thus higher activity of the soleus muscle fibers during treadmill running. This slow-to-fast muscle fiber shift was eventually confirmed by RNA sequencing of the soleus from control and trained mice, which will be described further below (see fig. IV.27).

\subsection{Remodeling of NMJ mitochondrial network by exercise}

Because MN properties tightly match the properties of the muscle fibers they innervate, if exercise elicits changes in muscle fiber type composition, MNs are expected to correspondingly adapt their properties (Kernell et al., 1999). MNs are particularly large and active cells with a high energetic demand at locations distant from the cell body. Indeed, motor axon terminals are likely the compartments of highest energy demand of the MN (Harris et al., 2012; Palay, 1956). Since tight regulation of mitochondrial biogenesis is generally important for meeting elevated energy demands in neurons, mitochondrial plasticity at the NMJ may adapt MN metabolic properties to increased energetic stress. In order to elucidate whether NMJ adaptations to exercise entail mitochondrial biogenesis, the modifications of the mitochondrial network organization was investigated in NMJs of MDO mice. MDO (Mito-Dendra2 ${ }^{\text {lox/wt; }}$ Olig2 $2^{\text {cre/wt) }}$ mice constitutively and selectively express the Dendra2 


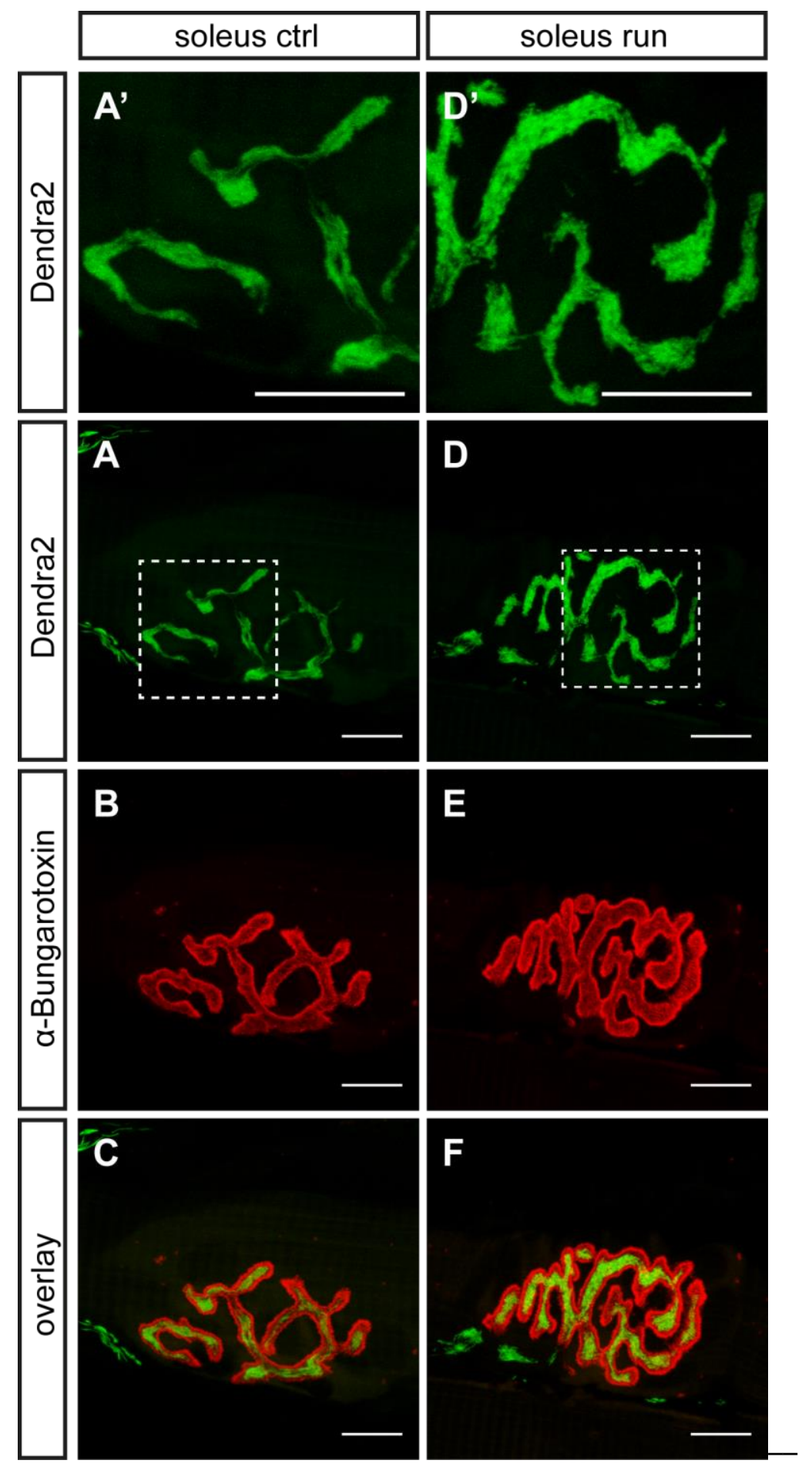

Figure IV.4. Soleus NMJs of sedentary and voluntary running MDO animals. Representative images of soleus NMJs of sedentary (A-C and A') and trained (D-F and D') mice. A and D. Presynaptic mitochondria are identified by endogenous fluorescence in MDO mice, which constitutively and selectively express Dendra2 in NM mitochondria. A' and D'. Closer views from the boxes in A and $D$ show the highly dense and interconnected mitochondrial network in both control and running soleus NMJs. B and E. The post-synapse was labeled with the fluorophore-conjugated $\alpha$-Bungarotoxin on the soleus muscle membrane. $\mathrm{C}$ and $\mathrm{F}$. The overlay of the pre-and post-synaptic compartments highlights the homogenous distribution of mitochondria in soleus endplates of both control and running animals. (Maximum intensity projections from confocal image stacks, scale bars: $10 \mu \mathrm{m}$ ) 
fluorescent protein in mitochondria of MNs, which allows direct investigation of the mitochondrial network in the different $\mathrm{MN}$ compartments. Thus Dendra2-labeled mitochondria were directly used as readout for MN adaptation to exercise. The postsynaptic compartment was identified using the labeling of the acetylcholine receptors (AChRs) on the muscle membrane by the fluorophore-conjugated non-toxic alpha subunit of the Bungarotoxin ( $\mathrm{aBtx}$ ).

Exercise was previously shown to have a different impact depending on the intrinsic properties of the motor unit component considered (Talmadge et al., 2004; Beaumont and Gardiner, 2002; Beaumont and Gardiner, 2003; Andonian and Fahim, 1988; Waerhaug et al., 1992; Deschenes et al., 1993). Therefore, different muscles and thus different motor-neuronal terminals were investigated. On the one hand, soleus muscle and the soleus motor pool are mainly constituted by slow and fast fatigue-resistant (S-FR) motor units, on the other hand, tibialis anterior (TA) muscle and the TA motor pool contain a majority of fast fatigable (FF) motor units, completed by fast resistant and fast intermediate (FR-FI) motors units.

\subsubsection{Characterization of NMJs from slow and fast muscles}

Figures IV.4 and IV.5 display representative images of soleus and TA NMJs from control and running MDO animals, obtained by maximal intensity projection of the initial confocal image stacks. In the soleus, NMJs of sedentary and trained mice occupied the same overall muscle fiber surface (fig. IV.4). However, the aBtx staining reveals more complex NMJ structures in running mice. They exhibited more, as well as longer or wider branches, which increased the area covered by the aBtx labeling (fig. IV.4B and E). Moreover, a closer view of the neuronal pre-synaptic mitochondria shows the highly dense and interconnected mitochondrial network in the soleus NMJs of both control and running mice (fig. IV.4A' and D'). The overlay of the preand post-synapse compartments highlights the homogenous distribution of mitochondria in both soleus endplates (fig. IV.4C and F). Qualitatively, an increase in the intensity of Dendra2 fluorescence was observed in the running compared to the control samples, possibly reflecting higher biosynthetic activity in the former NMJs (fig. IV.4A and D).

In the TA, NMJs of trained mice globally occupied a larger surface on the muscle fiber than NMJs of sedentary animals (fig. IV.5). In addition, NMJ structural 


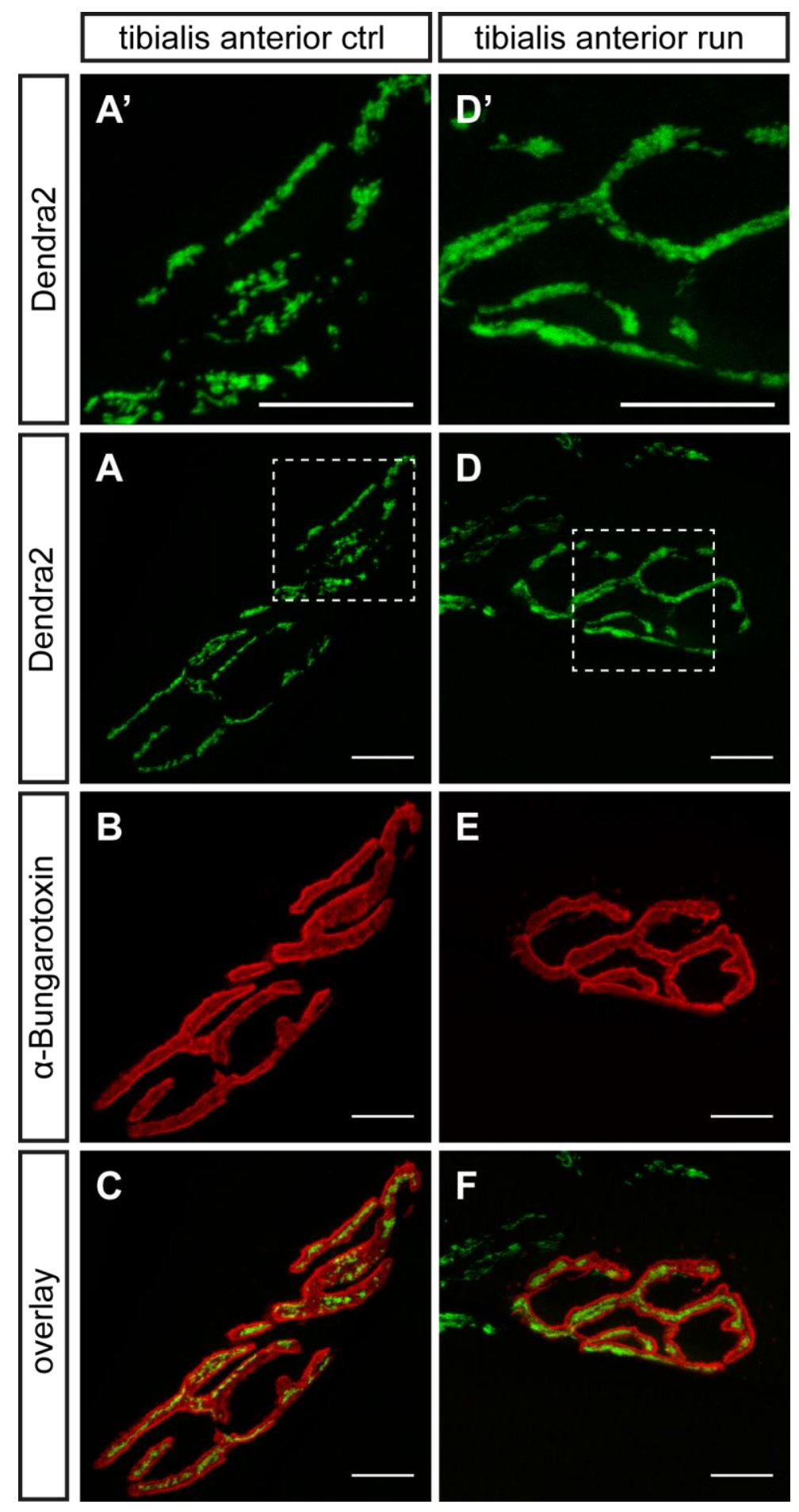

Figure IV.5. Tibialis anterior NMJs of sedentary and voluntary running MDO animals. Representative images of tibialis anterior NMJs of sedentary (A-C and $A^{\prime}$ ) and trained (D-F and $D^{\prime}$ ) mice. A and D. Pre-synaptic mitochondria are identified by endogenous fluorescence in MDO mice, which constitutively and selectively express Dendra2 in NM mitochondria. A' and D'. Closer views from the boxes in $A$ and $D$ show the fragmented mitochondrial network in the control NMJ and the interconnected mitochondrial network in the running NMJ of MDO mice. B and $\mathrm{E}$. The post-synapse was labeled with the fluorophore-conjugated $\alpha$-Bungarotoxin on the TA muscle membrane. $C$ and $F$. The overlay of the pre-and post-synaptic compartments highlights the scattered distribution of mitochondria in the control endplate and the more uniform distribution of mitochondria in the running synapse. (Maximum intensity projections from confocal image stacks, scale bars: 10 $\mu \mathrm{m}$ ) 
complexity appeared to be similar in both control and running animals (fig. IV.5B and E). However, a closer view of the neuronal pre-synaptic mitochondria showed a shift from a fragmented mitochondrial network in the control NMJ towards a more interconnected mitochondrial network in the running NMJs (fig. IV.5A' and D'). Unlike soleus NMJs, TA NMJs did not exhibit an obvious variation in the Dendra2 fluorescence intensity between the control and running samples (fig. IV.5A and D).

The high-resolution confocal image stacks of NMJs were reconstructed in three dimensions (3D). The reconstruction allowed to have a complete view of the NMJ structure and to have a precise and direct measurement of the pre- and postsynaptic NMJ components that took into account the curvature of the NMJ around the near-cylindrical portion of the muscle fibers. Only the Dendra2 signal that colocalized with the aBtx staining was considered for quantification. The Dendra2positive mitochondria that belong to the motor axon, but not to the NMJ itself, were removed from the 3D structures. Figure IV.6 presents the reconstruction of the representative synapses displayed in figure IV.4 and IV.5. In soleus of control and running animals, the NMJ mitochondria filled almost entirely the axon terminal in direct contact with the sarcolemma (fig. IV.6A and B). In contrast, the mitochondria clusters of TA NMJs appeared "scattered" when juxtaposed to the post-synaptic membrane (fig. IV.6C and D), confirming the observations from the projection images (see fig. IV.4 and IV.5). In control mice, soleus NMJs had few separate clusters, while larger numbers of disconnected clusters were identified in TA NMJs (fig. IV.6E and $G$ ). In running mice, few clusters were observed in soleus NMJs, while there were still many clusters in TA NMJs although they appeared less numerous than in control TA NMJs (fig. IV.6F and H). Endurance training resulted in the broadening of the cluster size range due to higher largest volumes of mitochondria clusters in NMJs of trained animals (fig. IV.6E-H).

\subsubsection{Exercise-induced adaptations of mitochondrial network in NMJs}

In order to describe NMJ morphology and characterize mitochondrial network, nine variables were measured and calculated from the reconstructed NMJs. The overall size of the NMJ was measured as the area of the aBtx staining on the sarcolemma. It represents the surface occupied by the synapse on the muscle fiber membrane. To further define the NMJ shape, the length of the NMJ branches were 


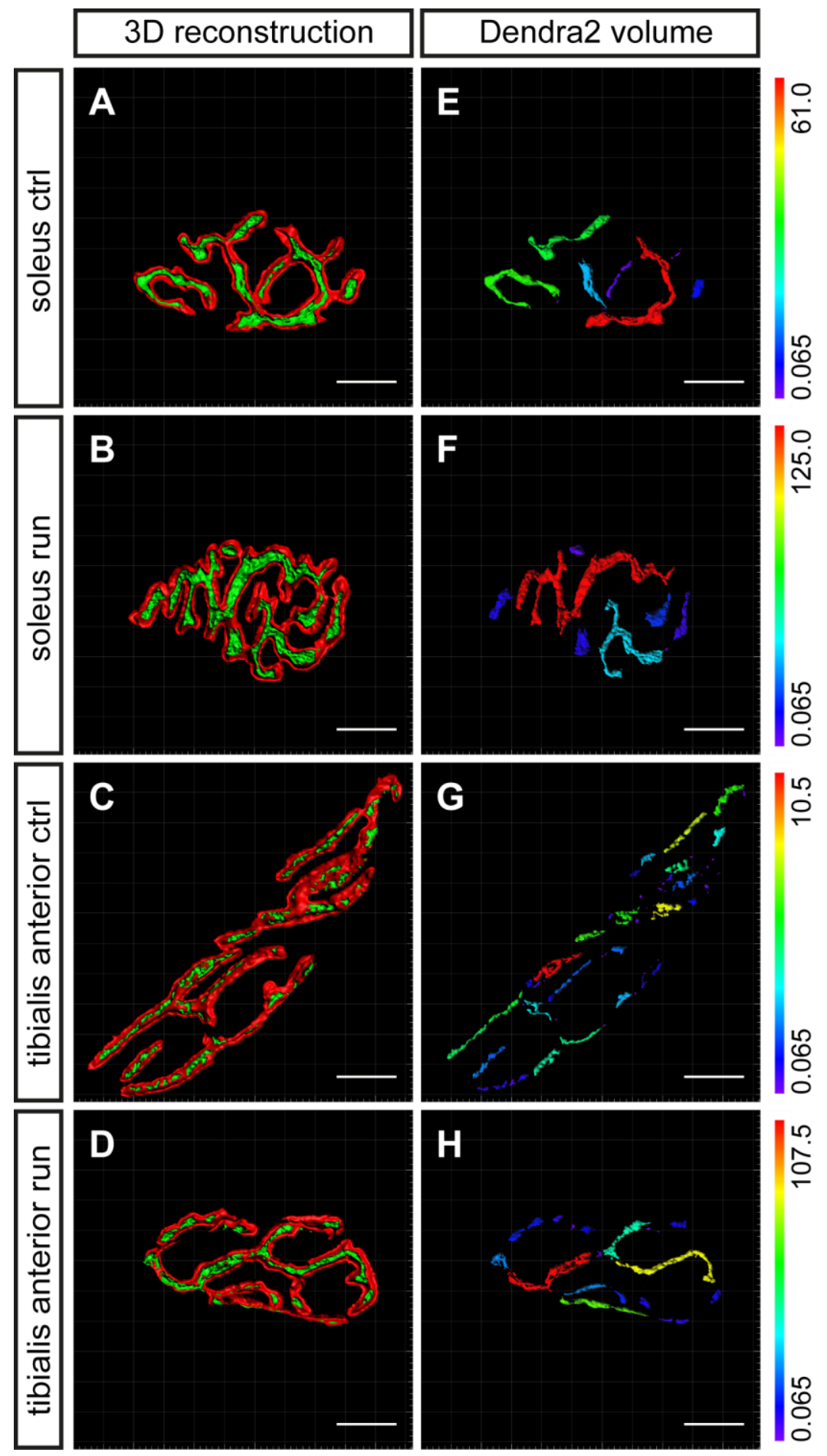

Figure IV.6. 3D reconstruction of soleus and tibialis anterior NMJs of sedentary and voluntary running animals enables precise measurements. A-D. The pre- and post-synaptic compartments of the soleus and tibialis anterior NMJs presented in fig. 4 and fig.5 are reconstructed in 3D. E-H. A colorcoding of the pre-synapse is used to visualize the volume of the different mitochondria clusters. (Color scale: red indicates the highest volume, purple indicates the lowest volume, the absolute extreme values $\left(\mu \mathrm{m}^{3}\right)$ are reported for each depicted NMJ; scale bars: $\left.10 \mu \mathrm{m}\right)$ 
measured. The area and the length measures were used to calculate the branchwidth index as the ratio of the total $\alpha \mathrm{Btx}$ area to the total branch length. The absolute volume of mitochondria in the pre-synaptic compartment was measured from the endogenous Dendra2 MN-specific mitochondrial labeling in the axon terminals. The mitochondrial volume was then normalized to the size of the NMJ. The ratio of the total mitochondrial volume to the total aBtx area gives an estimation of the mitochondria concentration in the NMJ. In the same way, the absolute number of distinct mitochondria clusters was automatically counted from the 3D reconstruction of the Dendra2 signal. The number of mitochondria clusters was also normalized to the size of the NMJ by dividing the total number of clusters by the total $\alpha \mathrm{Btx}$ area. The average volume of individual mitochondria clusters was calculated as the ratio of the total mitochondria volume to the total number of clusters. Because a fragmented network implies more clusters of rather smaller volumes, the average cluster volume, together with the normalized number of clusters, gives an indication on the distribution of the mitochondrial network in the NMJ, i.e. fragmented or connected. Finally, the average sphericity index of the mitochondria clusters was automatically calculated from the reconstructed NMJ structures. The index value results from the comparison of the cluster to a sphere of identical volume. The index ranges from 1 to 0 , indicating whether the cluster is close (tends to 1), or far (tends to 0) from a spherical shape. This last variable completes the description of the mitochondrial network by telling about the shape of the clusters, i.e. spherical or elongated/tubular.

Changes in the post-synapse were first investigated. In control mice, soleus NMJs were smaller than TA NMJs $(p<0.001)$ (fig. IV.7A). This was due to the shorter branch length in soleus $(p<0.001)$, although branch width was larger $(p<0.001)$ compared to TA NMJs (fig. IV.7B and C). Exercise induced the hypertrophy of soleus NMJs but the atrophy of TA NMJs. The post-synaptic surface was enlarged in soleus $(p<0.001)$, while it reduced it in TA NMJs $(p<0.001)$ (fig. IV.7D). This was paralleled by an increase in length of the post-synapse branches in soleus NMJs $(p<0.001)$ and a decrease in TA NMJs ( $p=0.003$ ) (fig. IV.7E). However, training did not affect the width of the post-synaptic branches in soleus $(p=0.910)$ nor in TA NMJs $(p=0.714)$ (fig. IV.7F). As a result, after the 12-week training, the NMJ area was larger in soleus compared to TA muscle $(p=0.001)$, due to larger branches $(p=0.001)$ but same branch length ( $p=0.997)$ (fig. IV.7A, B and C). 

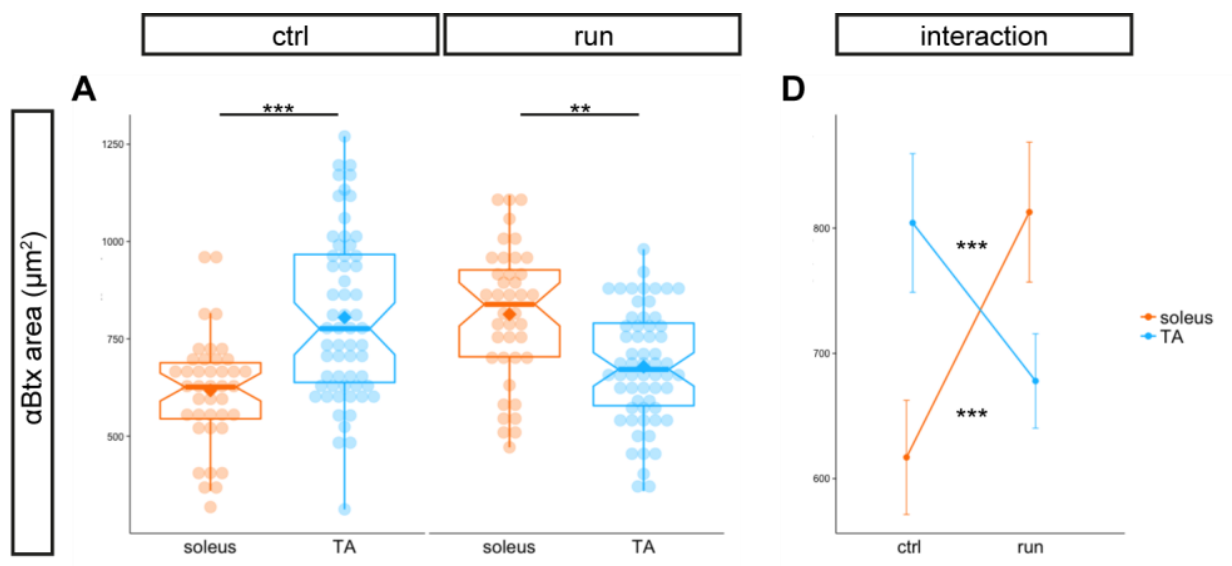

B

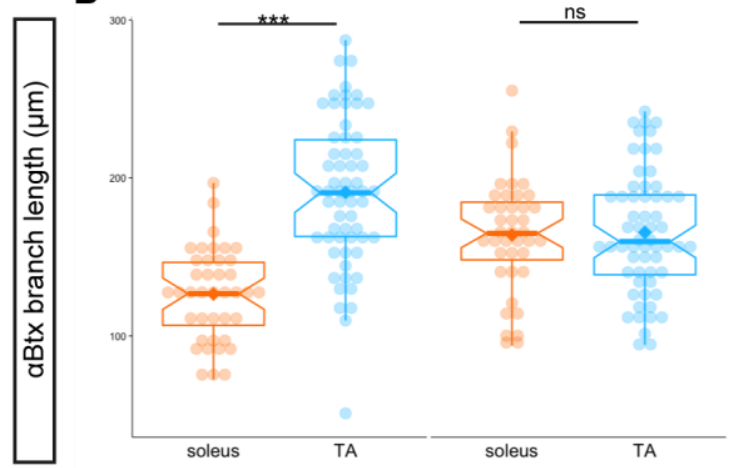

E

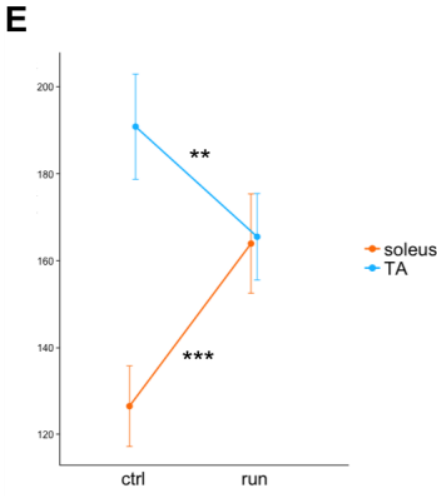

C
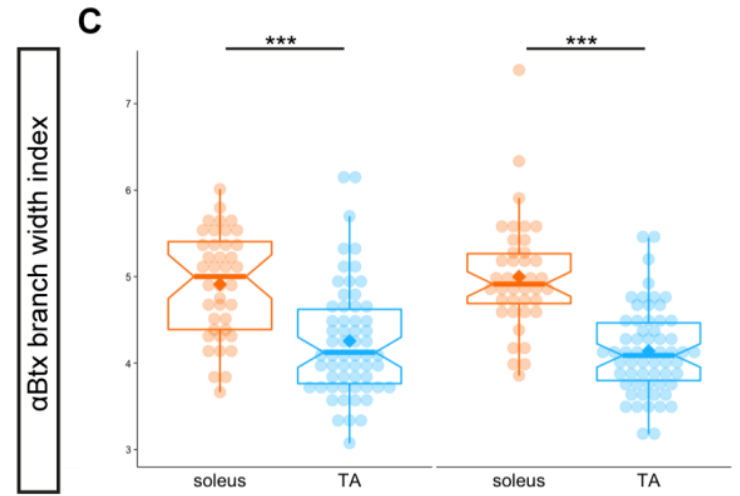

F

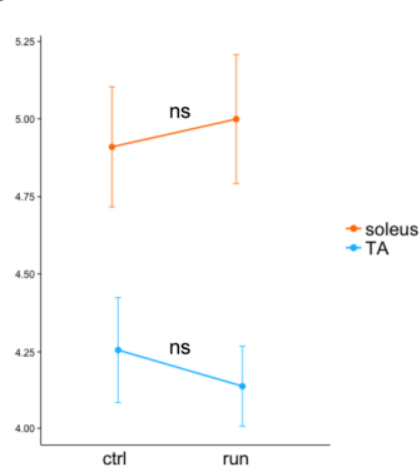

Figure IV.7. Upon exercise, the size of the post-synapse of soleus NMJs increases, while it decreases in tibialis anterior (TA) NMJs. A-C. Dot plots display individual group distribution. The overlaid box plot shows the median as a horizontal bar and the mean as a diamond shape. D-F. Interaction plots show the means of the groups to emphazise the effect of exercise on each muscle. A. The total area of the post-synapse was larger in TA NMJs of control mice, but was larger in the soleus NMJs of running animals. D. After running, the total post-synaptic area was enlarged in soleus NMJs, while it was reduced in TA NMJs compared to control. B. The total length of the post-synapse branches was shorter in soleus than in TA in NMJs of control but not running animals. E. Following training, the length of the post-synaptic branches was increased in soleus NMJs, whereas it was decreased in TA NMJs compared to control. C. Post-synaptic branches were larger in soleus than in TA NMJs in both control and running mice. F. However, the width of the post-synaptic branches remained unchanged after exercise in soleus as well as in TA NMJs compared to control. (ANOVA followed by Tukey's test with $\mathrm{Cl}=0.95$; significance: * $\mathrm{p}$-value $<0.05$, ${ }^{* *} \mathrm{p}$-value $<0.01$, ${ }^{* * *} \mathrm{p}$ value $<0.001$, ns: no significance; notches and error bars: $\mathrm{Cl}=0.95$; for each paradigm soleus: $\mathrm{n}=40$ and TA: $n=60$ NMJs from 4 MDO animals) 

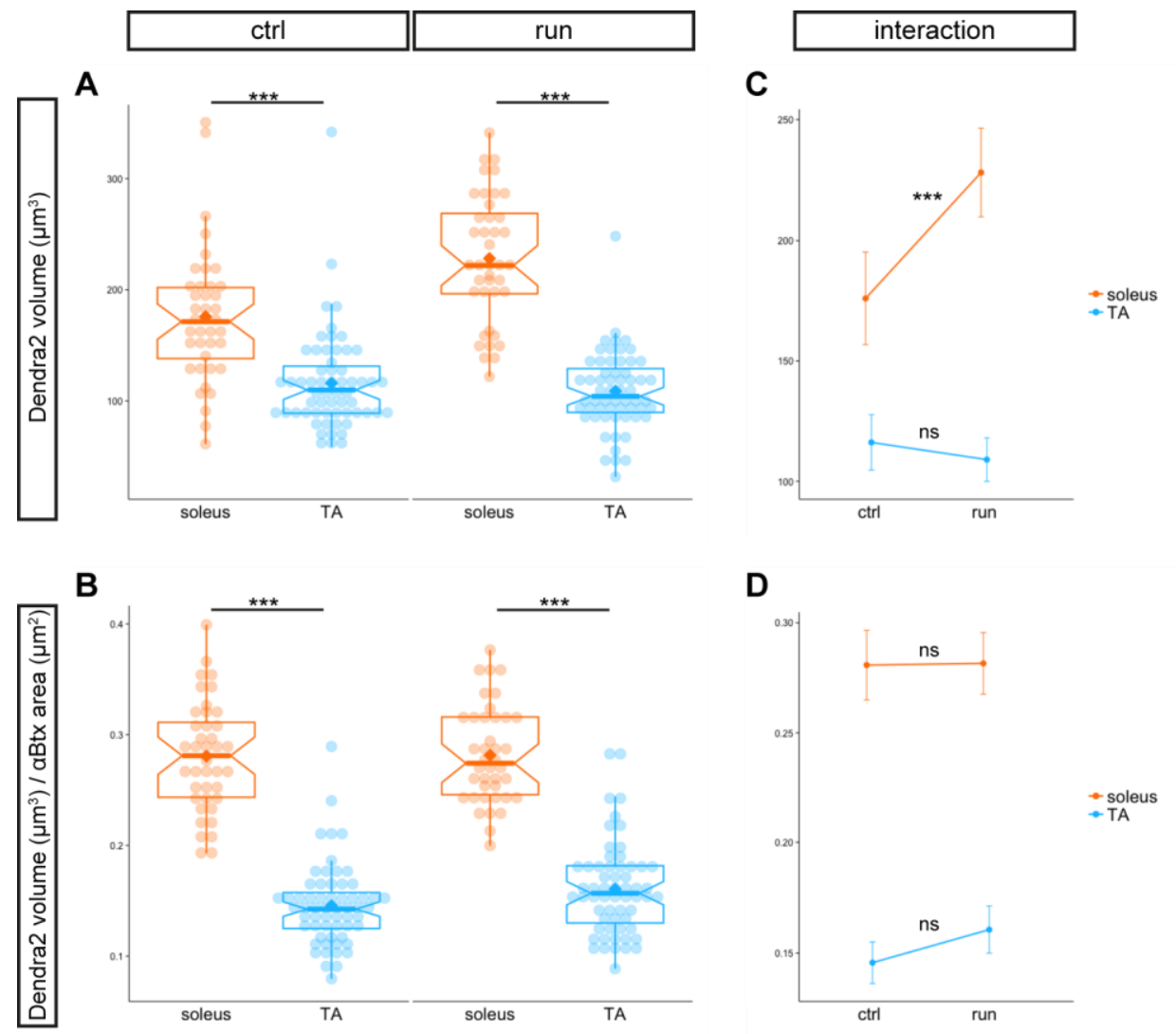

Figure IV.8. The presynaptic compartment of soleus NMJs contains a large volume of mitochondria that increases with exercise. A-B. Dot plots display individual group distribution. Overlaid box plot shows the median as a horizontal bar and the mean as a diamond shape. C-D. Interaction plots represent the effect of exercise on each muscle. A. The total mitochondrial volume was larger in soleus than in tibialis anterior (TA) NMJs in both control and running animals. C. Soleus NMJs had a larger mitochondrial volume in running compared to control mice, while it remained unchanged in TA NMJs. B. The total mitochondrial volume normalized to the NMJ size was larger in soleus than in TA NMJs in control as well as in running mice. D. However, when normalized to the NMJ size, the total mitochondrial volume was not different between running and control mice neither in soleus nor in TA NMJs. (ANOVA followed by Tukey's test with $\mathrm{Cl}=0.95$; significance: * $\mathrm{p}$-value $<0.05$, ${ }^{* *} p$-value $<0.01,{ }^{* * *} p$-value $<0.001$, ns: no significance; notches and error bars: $\mathrm{Cl}=0.95$; for each paradigm soleus: $n=40$ and TA: $n=60$ from 4 MDO animals) 
It was next examined whether the changes in NMJ size were accompanied by the modulation of the pre-synaptic mitochondrial network. The total mitochondrial volume was larger in soleus compared to TA NMJs $(p<0.001)$ in control animals (fig. IV.8A). Moreover, as soleus NMJs were smaller, the normalized mitochondrial volume was even larger in soleus compared to TA NMJs ( $p<0.001$ ) (fig. IV.8B). Thus soleus NMJs had a higher concentration of mitochondria than TA NMJs (1.93 fold). After 12 weeks of running, the absolute mitochondrial volume increased in soleus NMJs $(p<0.001)$ but not in TA NMJs $(p=0.851)$ (fig. IV.8C). However, when normalized to the NMJ size, the total mitochondrial volume was not different between running and control mice in soleus $(p=0.999)$ or in TA NMJs $(p=0.212)$ (fig. IV.8D). Thus the increase in mitochondrial volume in soleus is proportional to the increase in overall NMJ size area. In addition, the decrease in NMJ size in TA was not accompanied by an increase in mitochondrial concentration. As a consequence, the absolute mitochondrial volume as well as the normalized mitochondrial volume remained larger in soleus compared to TA NMJs after exercise (both $p=0.001$ ) (fig. IV.8A and B).

Furthermore, in control mice, the absolute total number of mitochondria clusters was larger in TA compared to soleus NMJs ( $p<0.001$ ) (fig. IV.9A), which was apparently not merely due to the larger sizes of the TA NMJs. Indeed, when normalized to the NMJ size, the number of mitochondria clusters remained larger in TA compared to soleus NMJs $(\mathrm{p}<0.001)$ (fig. IV.9B). Notably, the training induced a decrease in the number of mitochondria clusters of TA NMJs $(p<0.001)$, but not in soleus NMJs ( $p=0.497$ ) (fig. IV.9C). In addition, the normalized number of mitochondria clusters was also decreased in TA NMJs after running $(p=0.015)$, although there was no change in soleus NMJs ( $p=0.999)$ (fig. IV.9D). This result indicates that the reduction of mitochondria clusters is not only due to the reduction in TA NMJ area with exercise. Accordingly, both the absolute and relative number of mitochondria clusters remained larger in TA compared to soleus NMJs in running mice (both $\mathrm{p}=0.001$ ) (fig. IV.9A and B).

Finally, as described above in control mice, soleus NMJs exhibited a larger mitochondrial volume, but a smaller number of clusters (fig. IV.8A and IV.9A). In contrast, TA NMJs displayed a smaller mitochondrial volume but a larger number of clusters (fig. IV.8A and IV.9A). Thus, on average, mitochondria clusters of soleus NMJs had a larger volume compared to TA NMJs ( $p<0.001$ ) (fig. IV.10A). As shown 

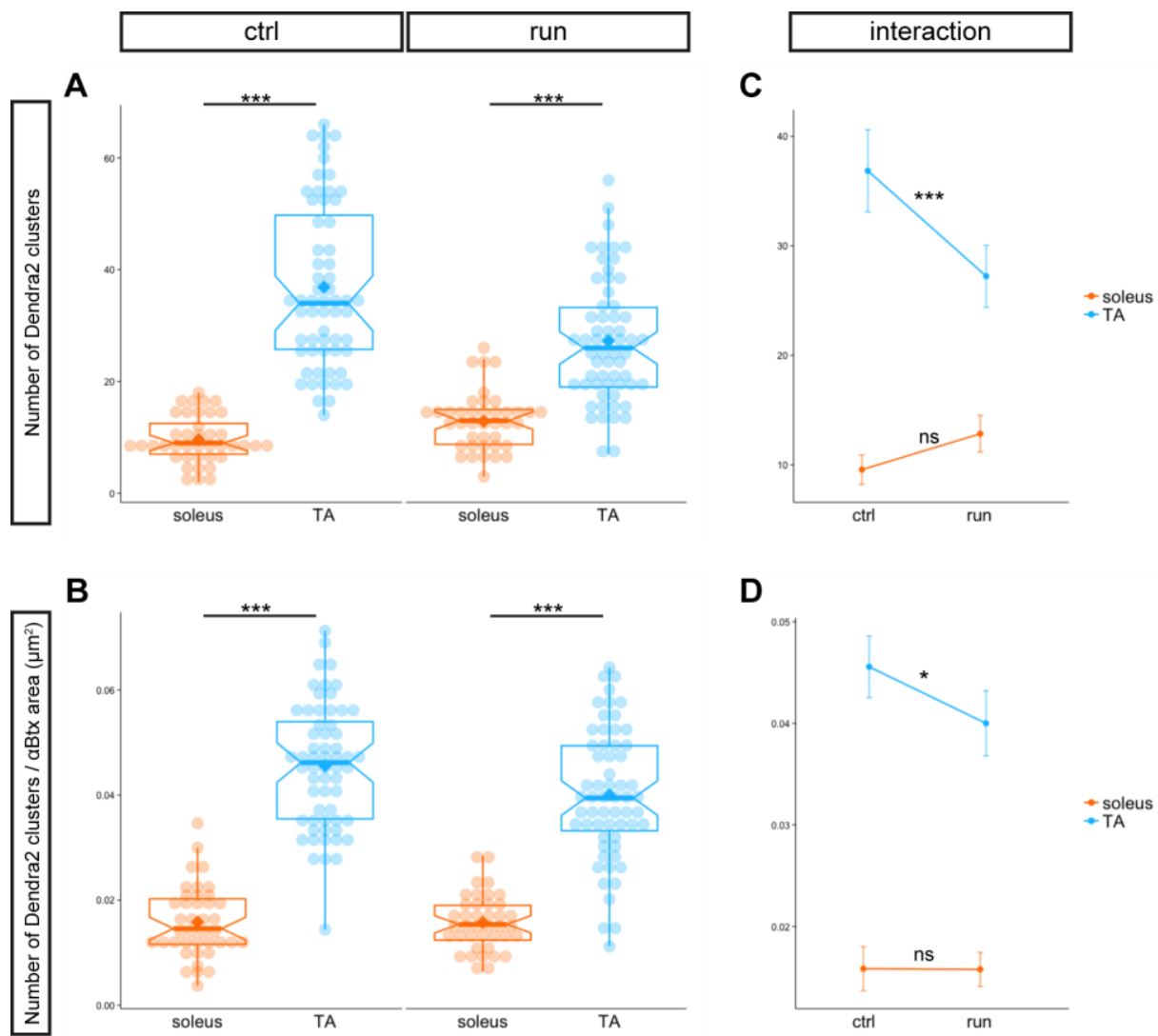

Figure IV.9. The presynaptic compartment of tibialis anterior (TA) NMJs contains a large number of mitochondria clusters, which decreases with exercise. A-B. Dot plots display individual group distribution. Overlaid box plot shows the median as a horizontal bar and the mean as a diamond shape. C-D. Interaction plots represent the effect of exercise on each muscle. A. The total number of mitochondria clusters was larger in TA than in soleus NMJs in both control and running mice. C. The number of mitochondria clusters was decreased in TA NMJs after running, but remained unchanged in soleus NMJs. B. The total number of mitochondria clusters normalized to the NMJ size was larger in TA NMJs compared to soleus in control as well as in running animals. D. While the total number of mitochondria clusters normalized to the NMJ size was decreased in TA NMJs after running, there was no change in soleus NMJs. (ANOVA followed by Tukey's test with $\mathrm{Cl}=0.95$; significance: * $\mathrm{p}$ value $<0.05,{ }^{* *} p$-value $<0.01,{ }^{* * *} p$-value $<0.001$, ns: no significance; notches and error bars: $\mathrm{Cl}=0.95$; for each paradigm soleus: $n=40$ and TA: $n=60$ from 4 MDO animals) 

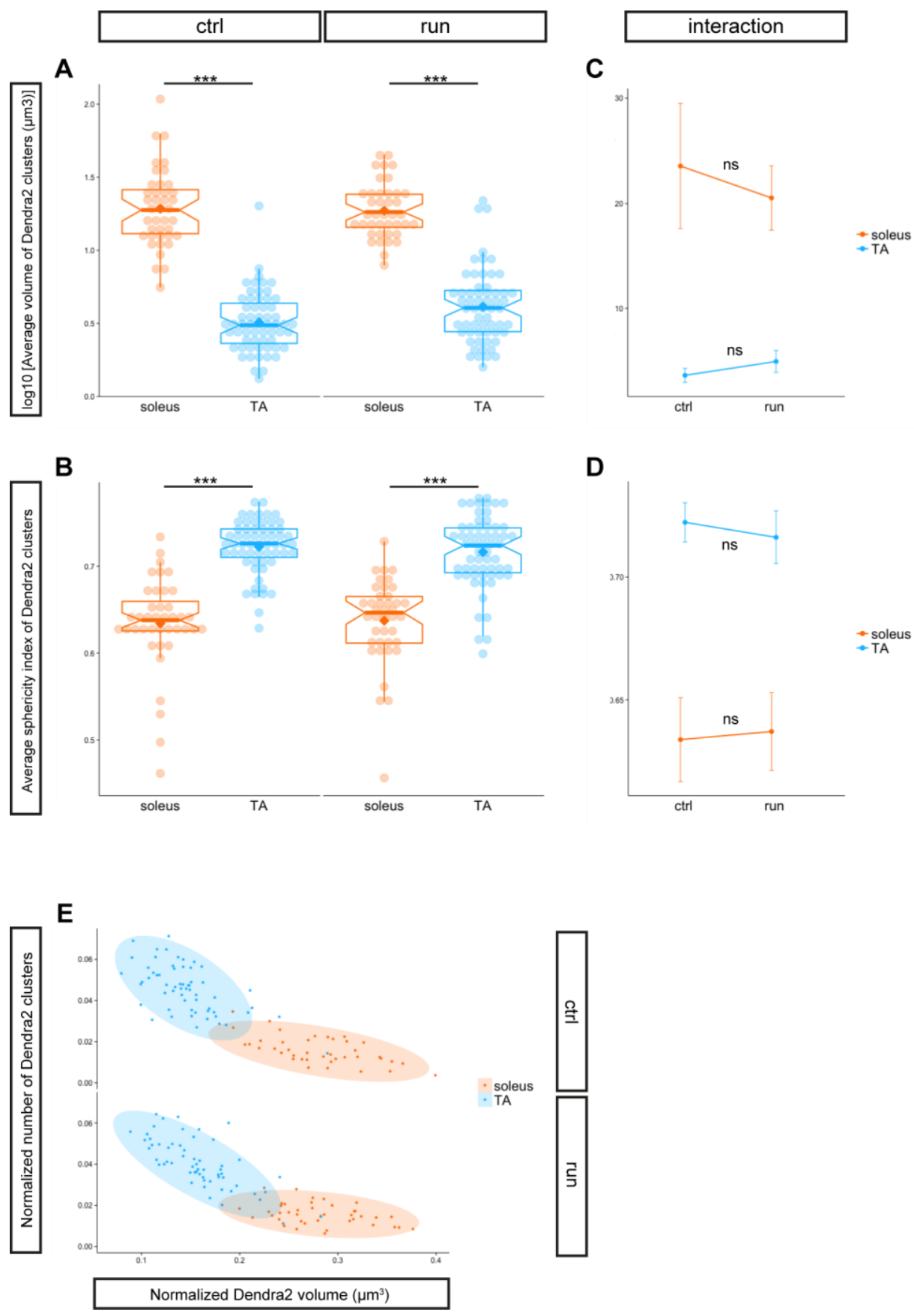

Figure IV.10. No modification of mitochondria cluster shape in the pre-synaptic NMJs of voluntary running mice. A-B. Dot plots display individual group distribution. Overlaid box plot shows the median as a horizontal bar and the mean as a diamond shape. C-D. Interaction plots represent the effect of exercise on each muscle. A. On average, mitochondria clusters of soleus NMJs had a larger volume than in tibialis anterior (TA) NMJs in both control and running mice. C. The average volume of mitochondria clusters was not different after running compared to control in both soleus and TA NMJs. B. Mitochondria clusters were more spherical in TA NMJs compared to soleus NMJs in control as well as in running animals. $D$. The average sphericity of mitochondria clusters was not altered by exercise neither in soleus nor in TA NMJs. E. Comparison of the relationship between the normalized mitochondrial volume and the normalized number of mitochondria clusters between NMJs of control and trained mice. (ANOVA followed by Tukey's test with $\mathrm{Cl}=0.95$; significance: * $\mathrm{p}$-value $<0.05,{ }^{*} \mathrm{p}$ value $<0.01,{ }^{* * *} p$-value $<0.001$, ns: no significance; notches and error bars: $\mathrm{Cl}=0.95$; for each paradigm soleus: $n=40$ and TA: $n=60$ from 4 MDO animals) 
before, exercise did not alter the normalized mitochondrial volume or the normalized number of clusters in soleus NMJs, which correlates with the absence of change in the average cluster volume ( $\mathrm{p}=0.993)$ (fig. IV.10C). This indicates a similar repartition of the mitochondrial network between soleus NMJs of control and running animals. Upon exercise, TA NMJs had a decreased normalized number of clusters but the normalized mitochondrial volume remained unchanged. However, there was no increase in average cluster volume $(\mathrm{p}=0.054)$ (fig. IV.10C). Thus, on average, mitochondria clusters remained larger in soleus than in TA NMJs in running mice $(p=0.001)$. These data correlate with the observation of more spherical mitochondria clusters in TA NMJs compared to soleus NMJs in control $(p<0.001)$ as well as in running $(p=0.001)$ animals (fig. IV.10B). The average sphericity index of mitochondria clusters was not affected by running exercise in soleus $(p=0.986)$ or TA NMJs $(p=0.866)$.

The normalized mitochondrial volume and the normalized number of mitochondria clusters appear to be the most prominent changes in NMJ mitochondrial network morphology upon exercise. Therefore, the relationship between these two variables was examined in control animals as well as in running animals (fig. IV.10E). NMJs exhibiting a high mitochondrial density had a connected network, while NMJs containing a low mitochondrial density displayed a fragmented network. Endurance training did not modify this relationship. The data suggest that the connectivity of the mitochondrial network is correlated to the density of mitochondria. When the mitochondrial volume increases in the endplate, the space is reduced and the chance that clusters become interconnected might be increased. The relationship between other measured variables was also studied in soleus and TA NMJs of sedentary and trained animals. Figure 11 represents the postsynaptic area, branch length and branch width index in relation to the mitochondrial volume (fig. IV.11A-D) or in relation to the number of mitochondria clusters (fig. IV.11 E-H). There was no significant change in the different relationships after exercise. However, it is interesting to note that soleus appear to be more affected by the modifications of the mitochondrial volume (fig. IV.11A-D), while TA was more affected by the change in number of clusters (fig. IV.11 E-H).

Taken together, these results indicate a fragmented network in TA NMJs, with small and spherical clusters. In contrast, in soleus NMJs, which have a higher mitochondrial concentration, mitochondria clusters are more elongated and 
A

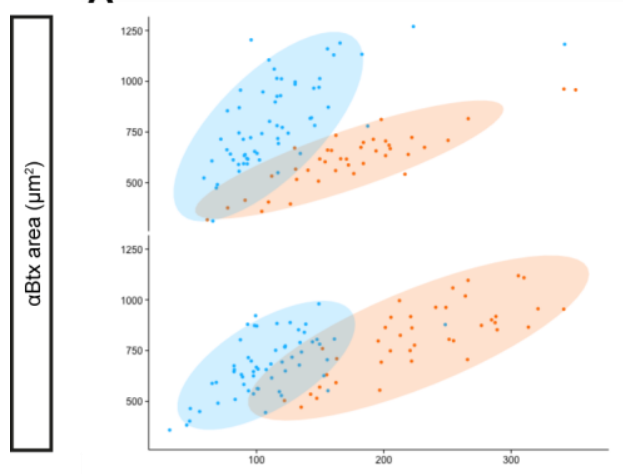

B
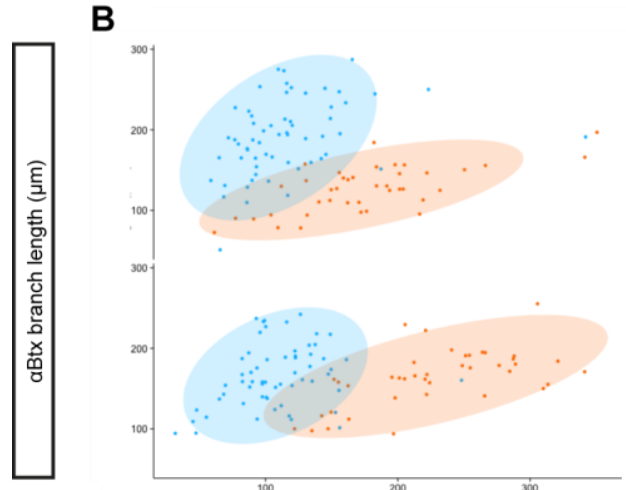

C

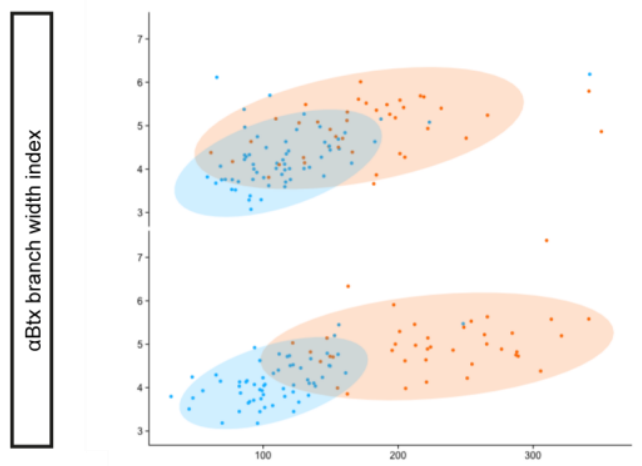

D

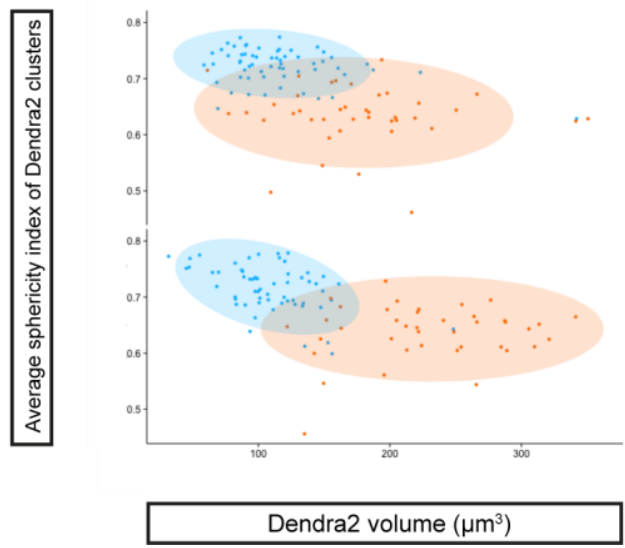

E

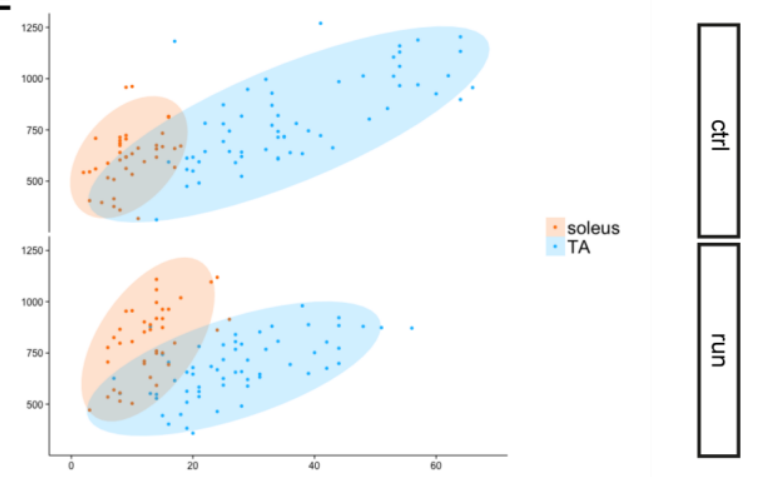

F

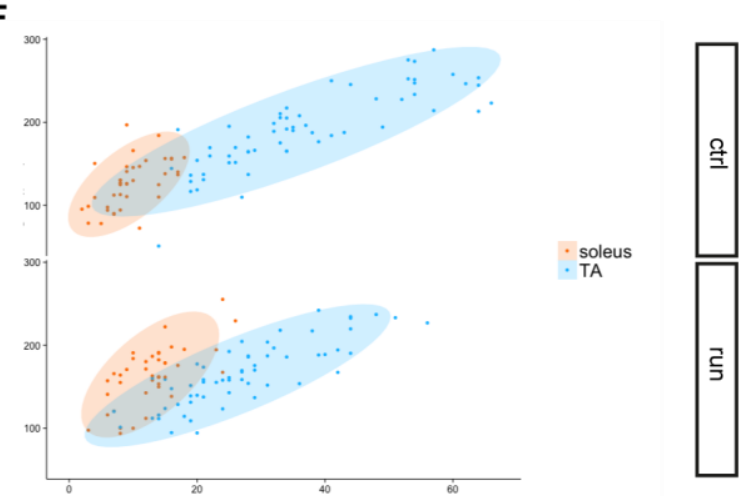

G

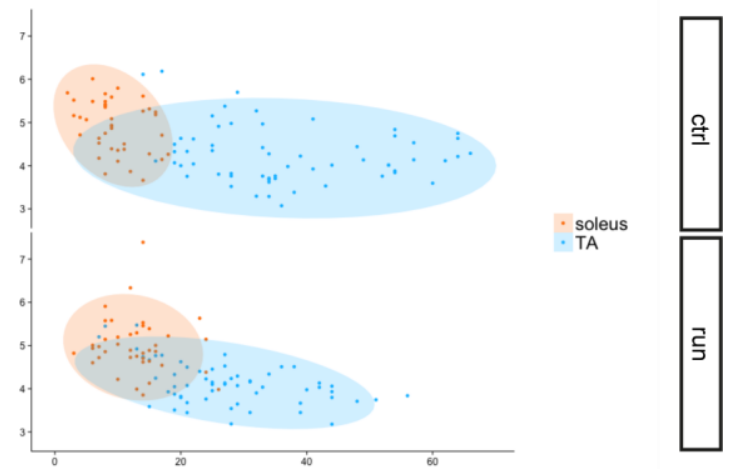

H

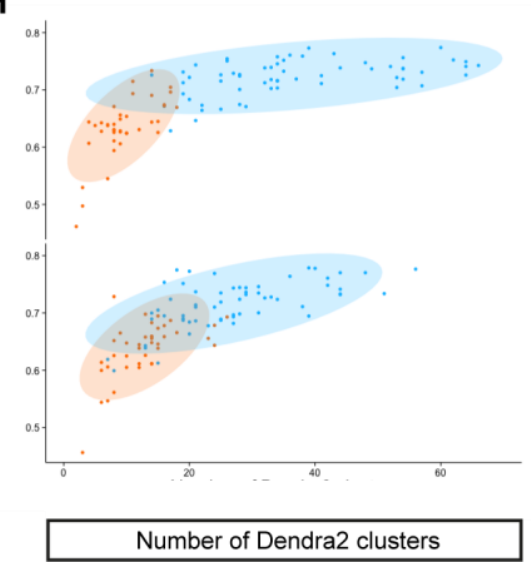

Figure IV.11. Voluntary running exercise does not strikingly affect the relationship between the measured variables in the NMJs of soleus and tibialis anterior (TA) muscles. Scatter plots represent the postsynaptic area, branch length and branch width index in relation to the mitochondrial volume $(A, B, C$ and $D)$ or in relation to the number of mitochondria clusters $(E, F, G$ and $H)$. (for each paradigm soleus: $n=40$ and TA: $n=60$ NMJs from 4 MDO animals) 
interconnected. Further, the data suggest that exercise induces a remodeling of the NMJ morphology that presents opposite characteristics between slow and fast muscles. Training elicits hypertrophy of slow post-synapses, while fast NMJs are reduced in size, due to a respective increase and decrease in branch length. In addition, enhanced activity maintains mitochondrial density in the pre-synapse by adjusting mitochondrial volume according to the change in the NMJ size. Finally, exercise reshapes mitochondrial network in fast NMJs in the direction of a more connected system. These results reinforce the idea that the important mitochondrial change in soleus NMJs is the increase in volume, whereas the important change in TA NMJs is the reduction in cluster number. The data also suggest that these changes may not just be adaptations of the pre-synapse to the change in postsynapse size, but rather specific modifications elicited in MNs in response to increased activity. Whether these changes are supported by differences in gene expression levels was further assessed using RNA sequencing.

\subsection{Transcriptional changes in muscle and MNs following long-term voluntary wheel running}

In order to identify gene expression changes accompanying or underlying the observed adaptations of muscles or NMJs to exercise, RNA sequencing was performed on samples from soleus muscle and lumbar spinal cord. Six trained C57BL/6J mice of similar running performance, and six corresponding sedentary controls were used for the transcriptomic analysis. During activities requiring low force levels only the slowest motor units are predicted to be active (Zajac and Faden, 1985). Thus, in the rather moderate intensity endurance training used (voluntary wheel running), a predominantly slow fiber-containing muscle such as soleus is likely much more recruited than the predominantly fast fiber-containing plantaris or tibialis anterior (TA) muscles. Therefore, the soleus was chosen for transcriptomic analysis after 12-weeks wheel running. As the effector of movements, muscle activity will immediately increase with exercise. However, the control of muscle contraction takes place in the spinal cord, which can be broadly divided in two different regions (Nógrádi and Vrbová, 2000; fig. 12B). The neurons and circuits generating motor output to the skeletal muscle, such as the motor neurons, are located in the ventral spinal cord. The dorsal spinal cord, in contrast, mainly contains neurons and circuits 
A

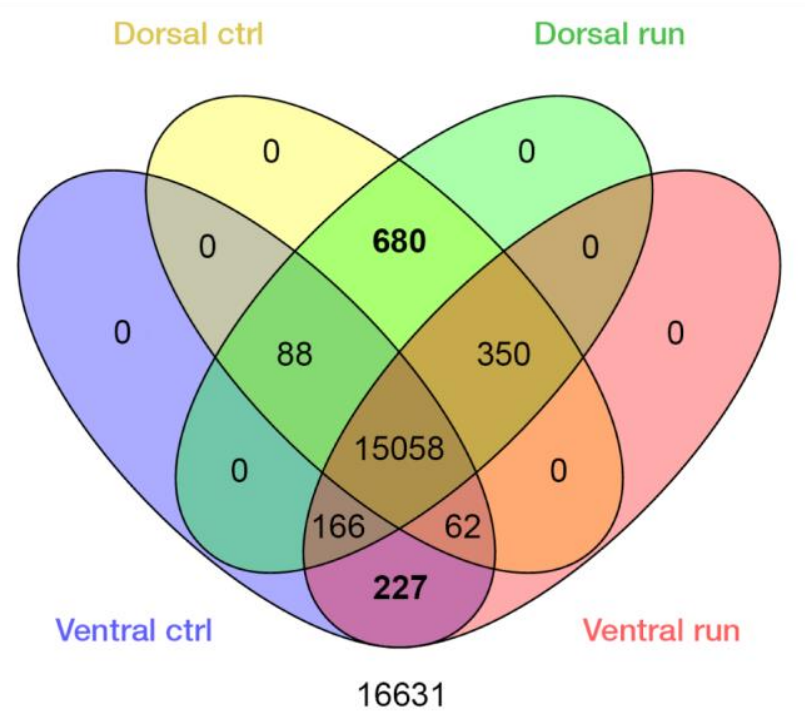

C

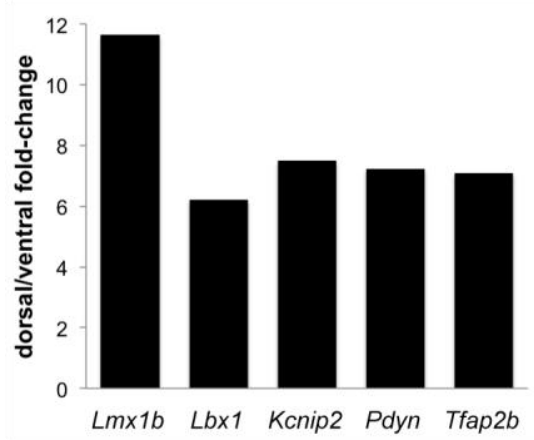

B

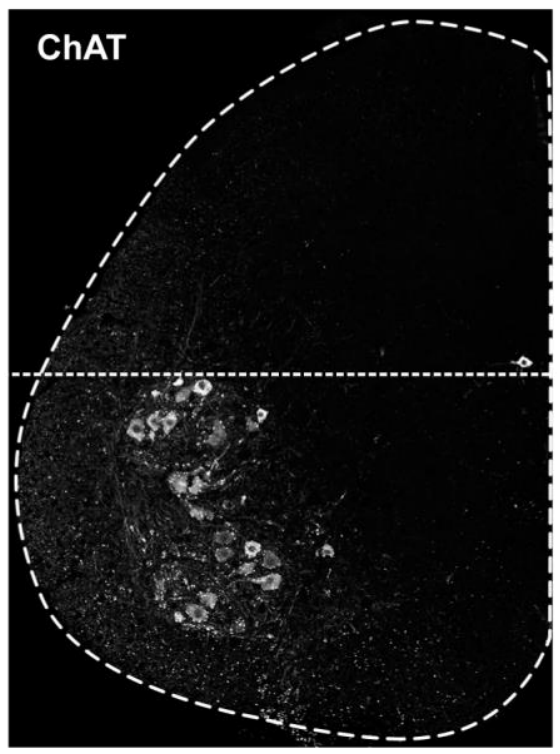

E

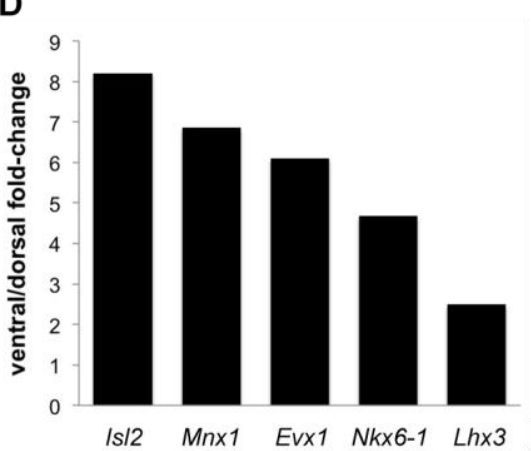

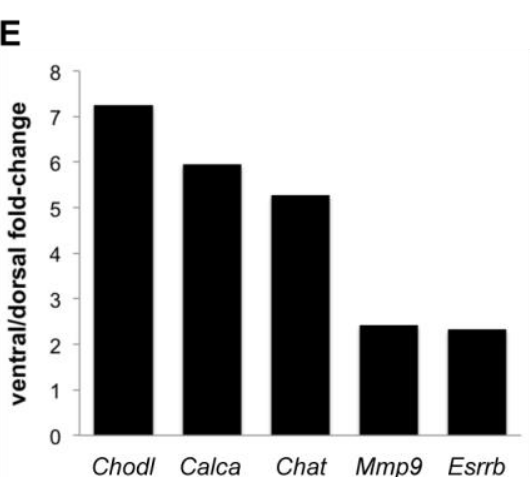

Figure IV.12. Accurate separation of the dorsal from the ventral region of the dissected spinal cords. A. A large number of genes are differentially expressed between the dorsal and the ventral region of the spinal cord ( $p$-value $<0.05$, fold-change $>2$ ), in control as well as in running mice. $B$. Choline-acethyltransferase (ChAT) staining of the cholinergic neurons in a hemi-cross section of a mouse spinal cord. The ChAT-positive MNs are specifically located in the ventral region. (The horizontal dotted line represents the separation of the spinal cord between the dorsal region at the top and the ventral region at the bottom). C-E. Dorso-ventral differential expression of identified neuronal markers. C. The dorsal spinal cord samples are highly enriched in genes either implicated in dorsal specification during development $(L m \times 1 b, L b \times 1)$, or expressed in differentiated dorsal interneurons (Tfap2b, Kcnip2 and Pdyn). D. The ventral spinal cord samples are enriched with genes that play a role in ventral early differentiation. E. MN markers are highly expressed in ventral compared to dorsal samples. 
processing and relaying somatosensory information, such as received by mechanoreceptors embedded in muscles, tendons or skin. Both ventral motor and dorsal sensory networks will experience increased activity upon exercise, which however may differ because of their involvement in primarily generating motor output or sensory input, respectively. Therefore, the dorsal and ventral regions of the lumbar spinal cord were isolated and separately processed for transcriptome analysis via RNA sequencing.

\subsubsection{Assessing sample quality and reliability}

The expression profile of dorsal versus ventral spinal cord samples were first characterized in all 12 mice to confirm the accurate separation of the dorsal from the ventral region during sample collection. A large number of genes were differentially expressed between the dorsal and the ventral horn of the spinal cord, in control as well as in running mice (fig. IV.12A). Among the 16631 genes commonly expressed by all four groups of samples, 680 genes were specifically enriched in the dorsal samples and 227 were specifically enriched in the ventral samples. These distinct pools of genes were then screened for identified neuronal markers. The dorsal spinal cord samples were highly enriched in genes such as $L m \times 1 b$ and $L b x 1$ that are implicated in dorsal horn specification during development (fig. IV.12B; Alaynic et al., 2011). Dorsal samples also had high expression of markers for differentiated dorsal interneuron types, including Tfap2b, Kcnip2 and Pdyn (fig. IV.12C; Wildner et al., 2013). In contrast, the ventral spinal cord samples were enriched with genes required for ventral horn cell type diversification, including Mnx1 (also known as Hb9), Isl2, Nkx6-1 and Lhx3 (fig. IV.12D; Alaynic et al., 2011). Furthermore, MN markers were expressed at higher levels in the ventral compared to the dorsal spinal cord samples, which included Chodl, Calca, Chat, Mmp9 and Esrrb (fig. IV.12E; Forsgren et al., 1993; Piehl et al., 1993; Enjin et al., 2010; Kaplan et al., 2014; Friese et al. 2009). This is illustrated by the labeling of cholinergic MNs and V0c interneurons in the ventral spinal cord using an antibody against ChAT (choline-acethyltransferase, the Chat gene product) (fig. IV.12B).

It was next important to assess the efficacy of the training of the mice. Gene expression in the soleus muscle and ventral spinal cord was profoundly altered after 12 weeks of running (fig. IV.13). The 50 most significantly up-regulated genes and 
A

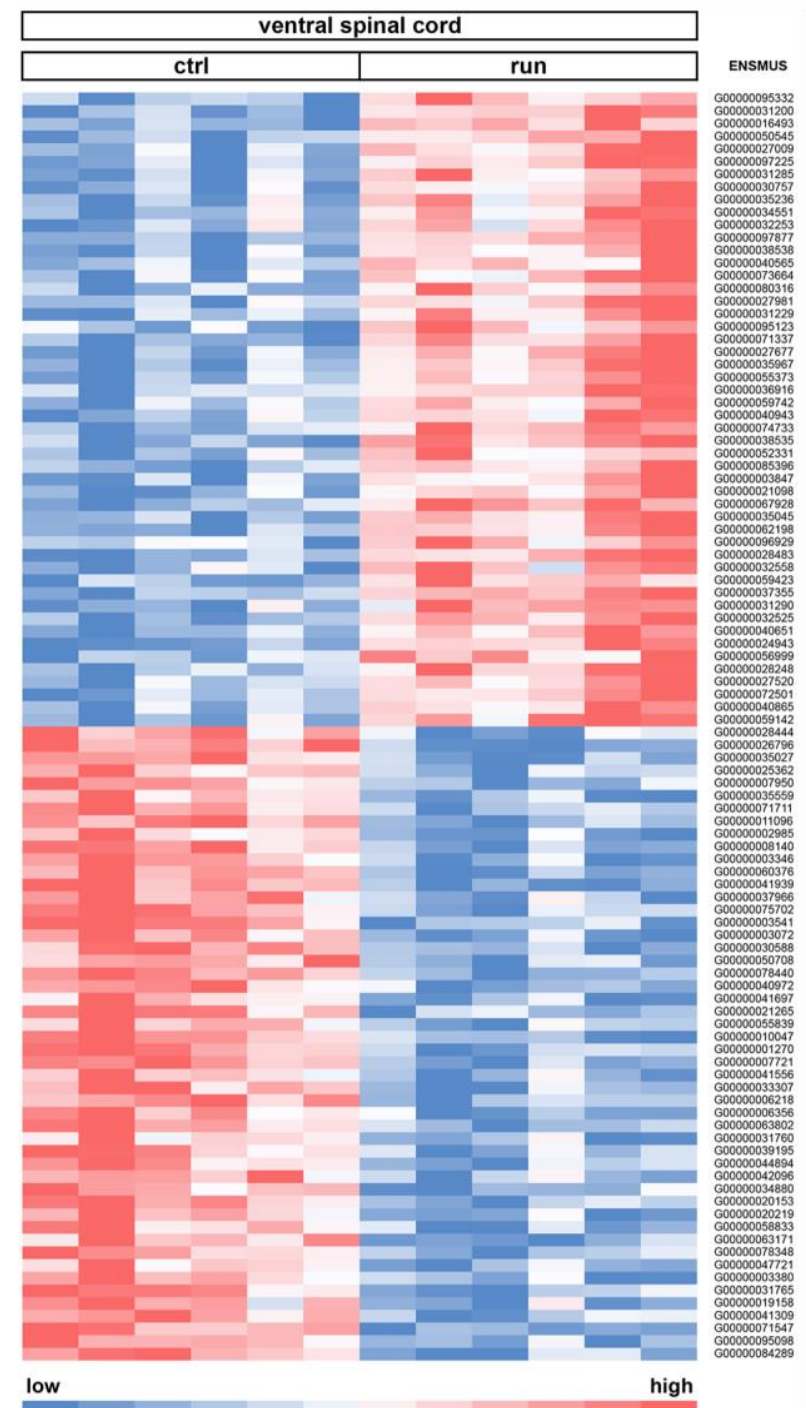

B

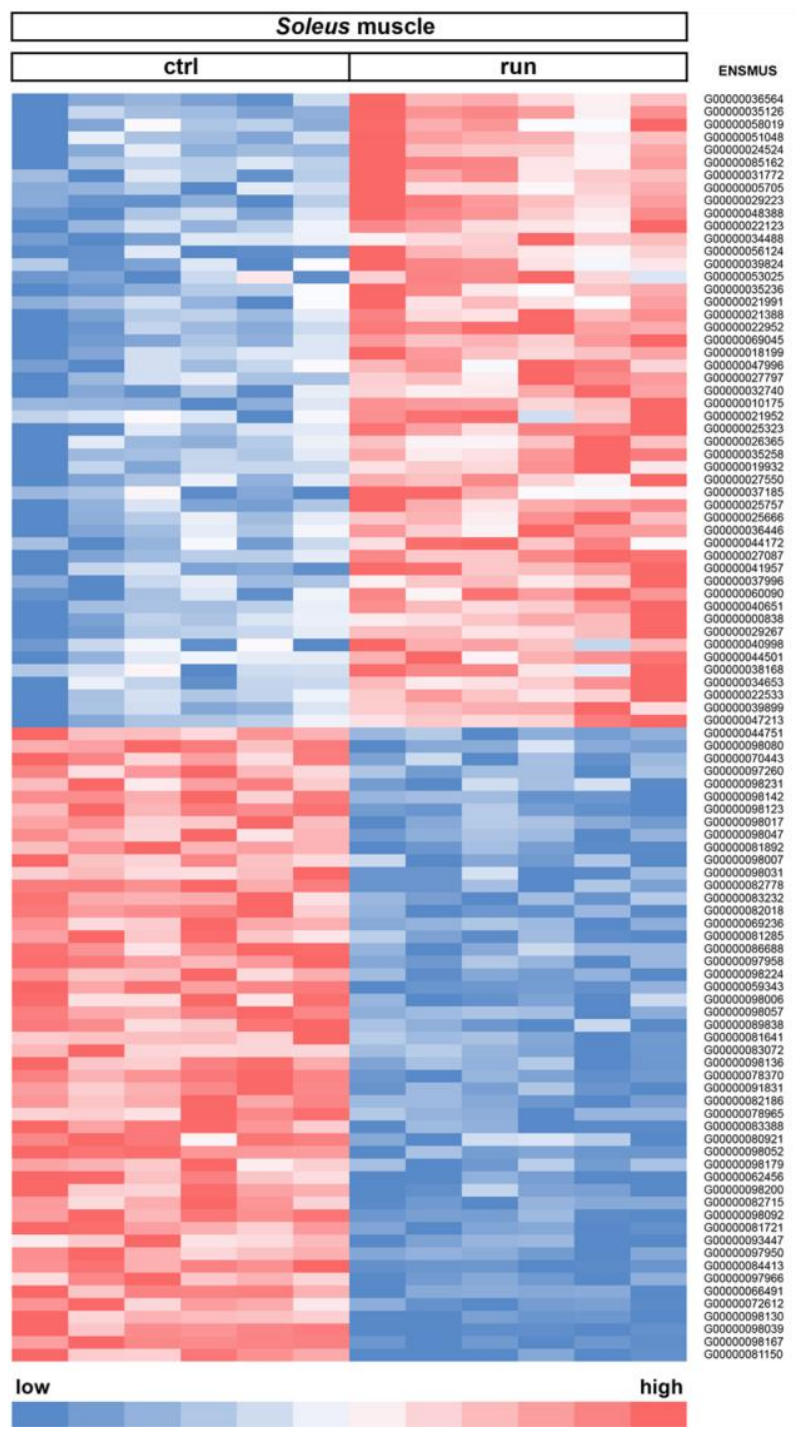

Figure IV.13. Long-term voluntary running alters the gene expression profile of the soleus and the ventral spinal cord. A-B. The 100 most significantly differentially regulated genes in the ventral spinal cord and in the soleus muscle after 12 weeks of endurance training. A. The ventral spinal cord gene expression profile is significantly different between sedentary and trained animals. B. Training also significantly impacted soleus gene expression profile. (Heat maps: $p$-value $<0.001$ and ranking by fold-change; color code: red as highest and blue as lowest gene expression level; $n=6 \mathrm{C} 57 \mathrm{BI} / 6 \mathrm{~J}$ mice per group) 
the 50 most significantly down-regulated genes following exercise - according to the p-value - show very different levels of expression between the control and the running samples in both the soleus and the ventral spinal cord.

\subsubsection{Common transcriptional changes in muscle and spinal cord}

Following the RNA sequencing experiment, a computational functional analysis using the Gene Ontology database was performed in order to enable the functional interpretation of the experimental data. The Gene Ontology (GO) annotations are used to classify functional attributes of gene products as of Biological Process, Molecular Function and Cellular Component.

GO taxon

Biological Process

Molecular Function

Cellular Component

\section{what the associated GO terms describe}

biological functions in which a gene product is involved

activities and functions that a gene product executes

subcellular or extracellular localization of a gene product

The analysis is based on the ranking of the genes according to their $p$-value and fold-change of expression, followed by a refinement method to identify the GO terms enriched in the data (see Materials and Methods section). Figures IV.14-25 display the GO terms in each of the three taxonomies, which representation in the dataset was significantly altered by chronic endurance exercise. By means of a color code, the graphs also indicate whether the effect is positive (e.g. activation of the function) or negative (e.g. repression of the process). In all tissues sampled, a broad range of functional changes occurred, but the ventral spinal cord showed most alterations upon training. The possibility of a common adaptation of the different parts of the neuromuscular system to exercise was first investigated. To achieve this, the results from the muscle and spinal cord samples were compared in order to identify similarly regulated biological processes, molecular functions and cellular components across the three tissues (fig. IV.26). Then, the processes, functions and components shared by the three tissues were screened for similar changes in the expression of particular genes (table IV.3).

Training first resulted in enhanced activity in the nucleus as suggested by the increased regulation of transcription, spliceosomal assembly and nucleic acid binding 


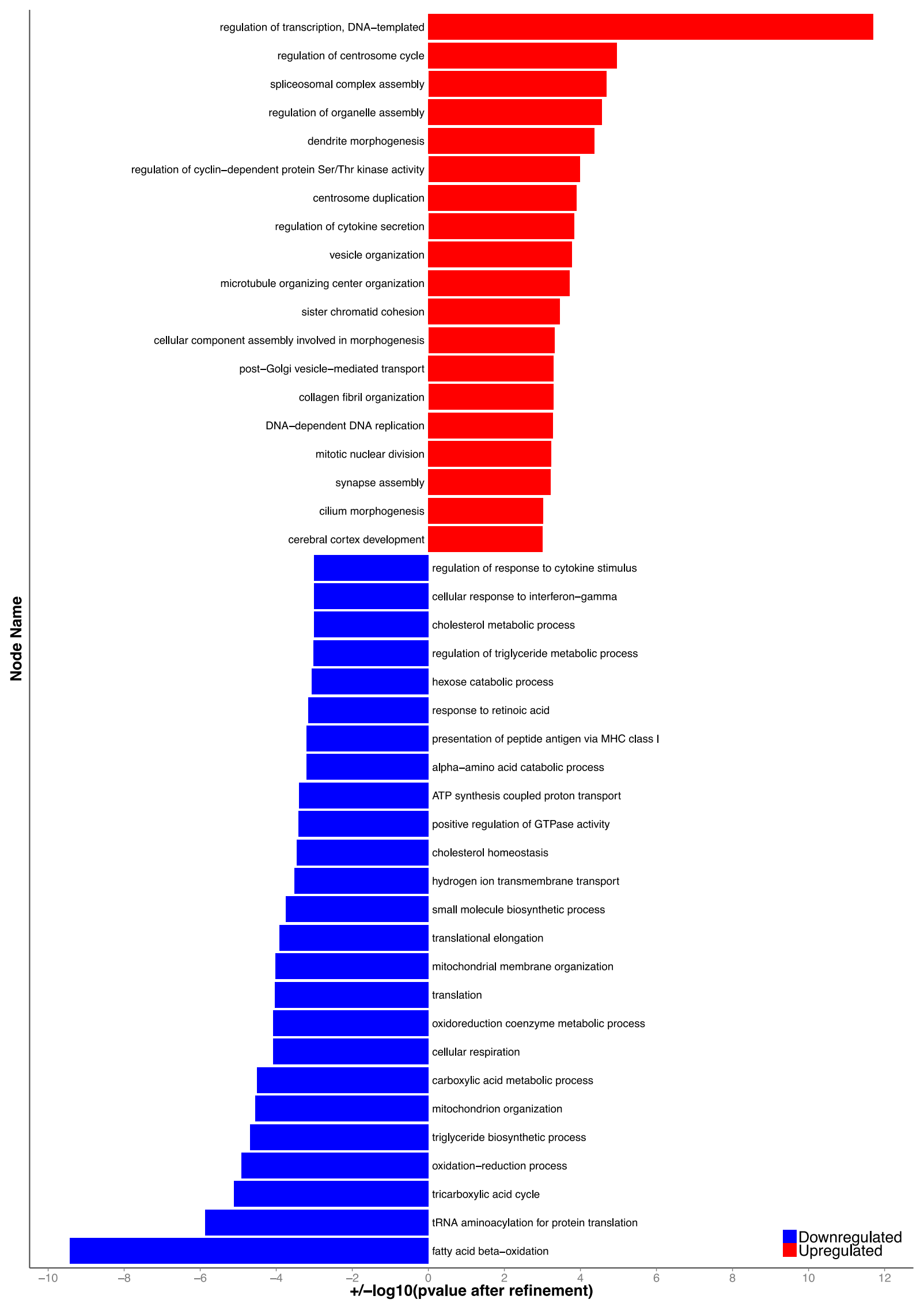

Figure IV.14. Biological processes activated or repressed upon exercise training in the soleus muscle. (GO terms enrichment after computational functional analysis; $p$-value after refinement $<0.5$ ) 


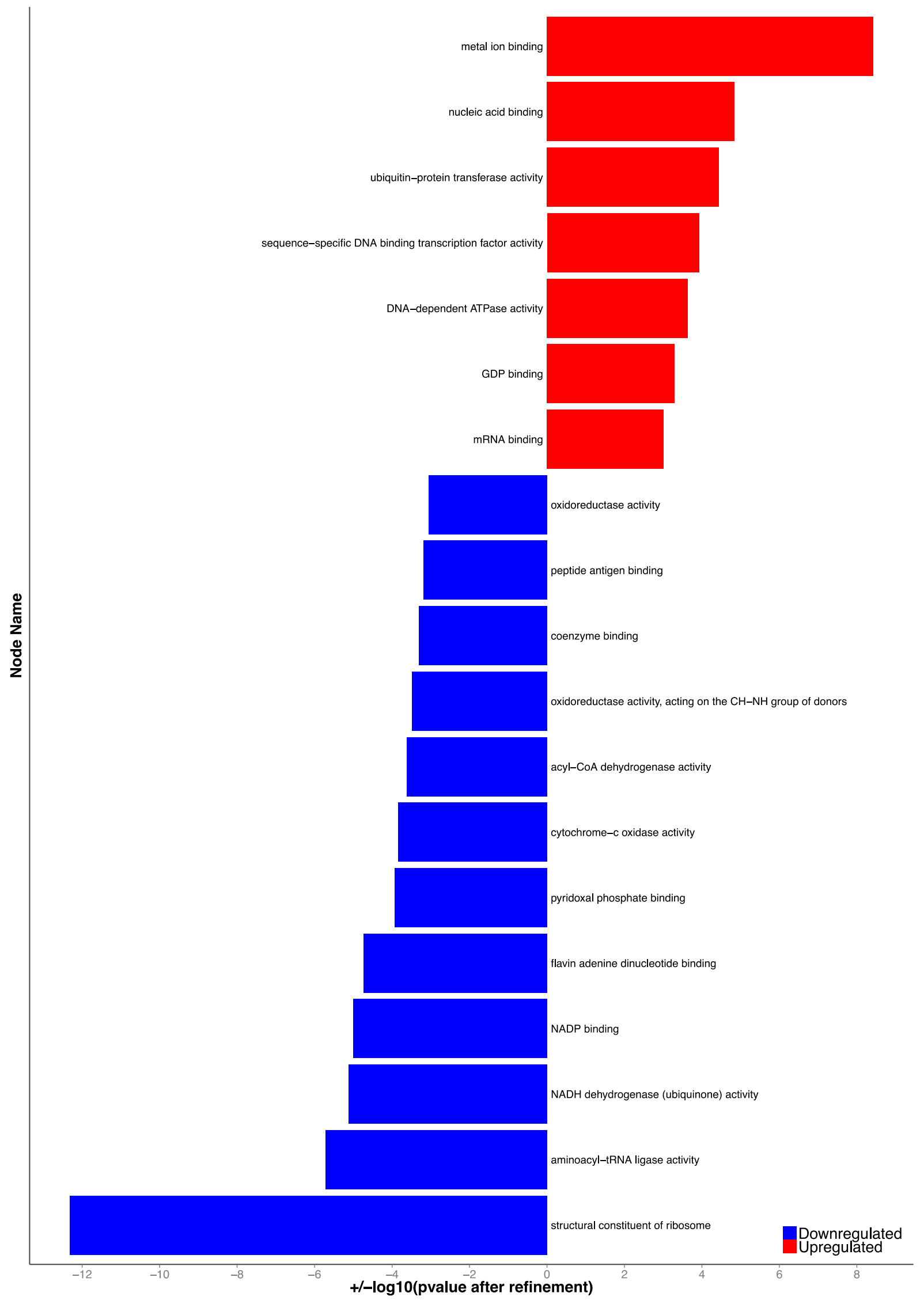

Figure IV.15. Molecular functions activated or repressed upon exercise training in the soleus muscle. (GO terms enrichment after computational functional analysis; $p$-value after refinement $<0.5$ ) 


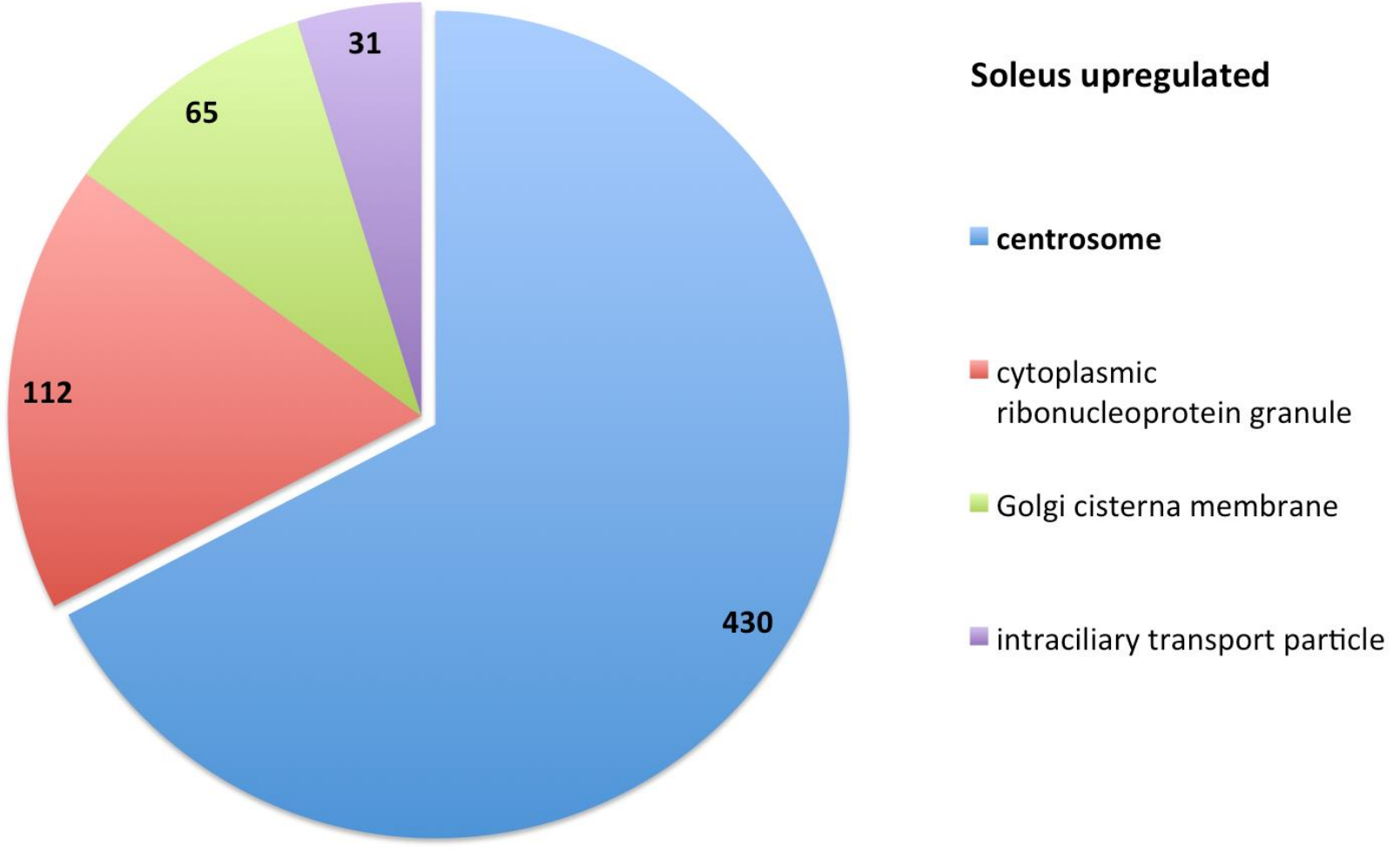

Figure IV.16. Exercise training mainly activated the centrosomal compartment in the soleus muscle. (GO terms enrichment after computational functional analysis; $p$-value after refinement $<0.5$; number of genes expressed in soleus, which product localizes in the compartment) 
A

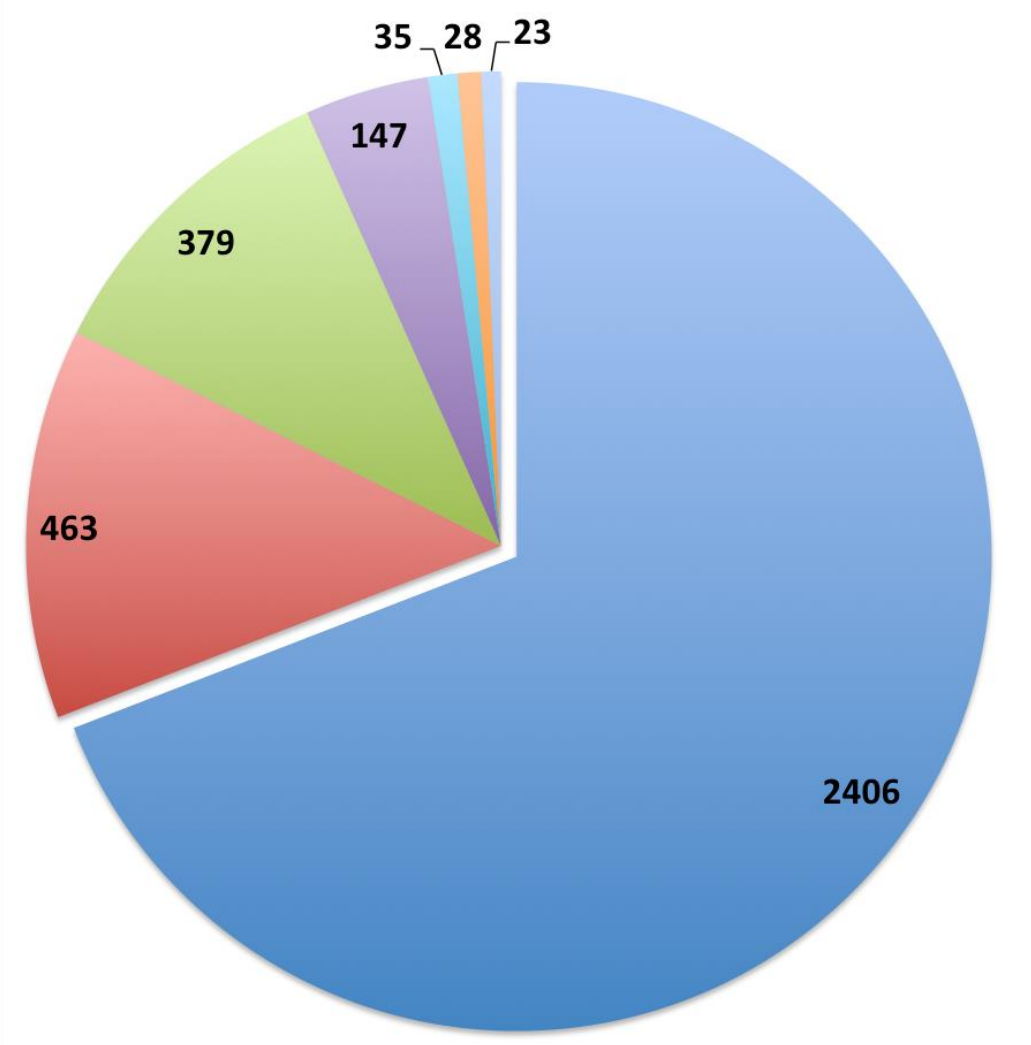

\section{Soleus downregulated}

mitochondrion

ribosome

actin cytoskeleton

- organelle outer membrane

MHC class I protein complex

proton-transporting twosector ATPase complex

proteasome accessory complex

B

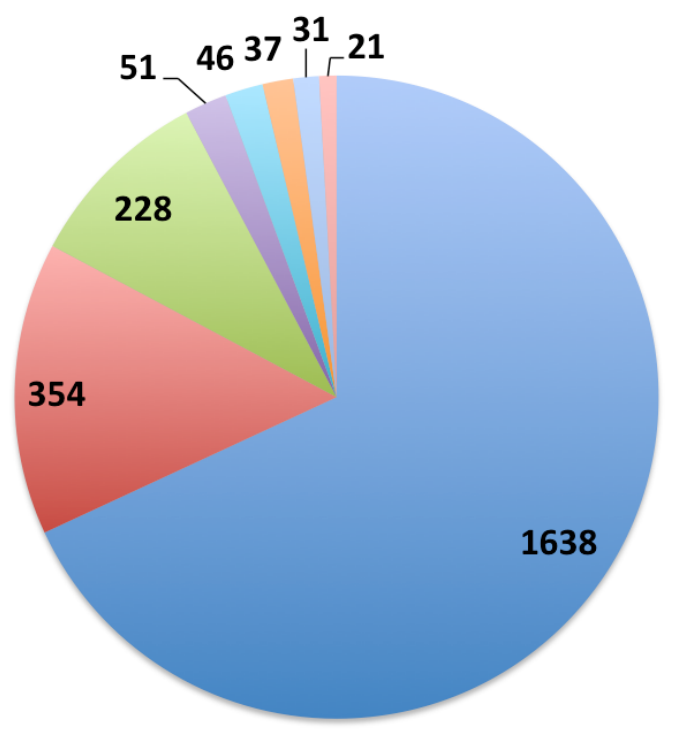

Soleus downregulated mitochondrion

mitochondrion

mitochondrial inner membrane

mitochondrial matrix

mitochondrial respiratory chain

mitochondrial ribosome

mitochondrial nucleoid

mitochondrial respiratory chain complex I

mitochondrial proton-transporting ATP synthase complex

Figure IV.17. Exercise training mainly repressed activity of the mitochondrial compartment in the soleus muscle. A. Principal cellular components which activity is repressed upon exercise. B. Detail of the mitochondrial sub-compartments. (GO terms enrichment after computational functional analysis; $p$-value after refinement $<0.5$; number of genes expressed in soleus, which product localizes in the compartment) 
activity (fig. IV.26). For instance, the gene expression of the splicing factor SRSF1 (serine/arginine-rich splicing factor 1) and of the short isoform of the chromatinassociated protein PSIP1 (PC4 and SF2 interacting protein 1) were increased. PSIP1 might act to modulate splicing through its binding to both chromatin and splicing factors such as SRSF1, which relocalizes into the cytoplasm in response to stress and couples transcription with alternative splicing (table IV.3; Pradeepa et al., 2012; Cramer et al., 1999). Exercise also activated the expression of the splicing regulator PTBP2-encoding gene (polypyrimidine tract binding protein 2), which inhibits a set of adult neuronal exons to promote embryonic neuronal maturation (table IV.3; Licatalosi et al., 2012; Li et al. 2014).

In addition, training regulated the expression of transcription factors that have mitochondrial genes as targets. The up-regulation of Mef2a (myocyte enhancer factor-2a) was paralleled by the down-regulation of one of its target gene and interactor Hdac5 (histone deacetylase 5), indicating the modulation of the transcription of genes implicated in cellular homeostasis (table IV.3; Czubryt et al., 2003). Notably, the transcription of the transcriptional cofactor PGC-1a (peroxisome proliferator-activated receptor $\mathrm{\gamma}$ coactivator-1 $\alpha$ ), a master regulator of mitochondrial biogenesis and fatty acid oxidation, is activated by MEF2 and repressed by HDAC5 (Czubryt et al., 2003). Moreover, PGC-1a activates the transcription of Esrra (estrogen-related receptor $\alpha$ ), the gene encoding its coactivator ERR1. The gene expression of ERR1, which regulates target genes involved in energy metabolism (glycolytic pathway, pyruvate metabolism, and tricarboxylic acid cycle), was downregulated after training (table IV.3; Deblois and Giguère, 2011).

Furthermore, chromatin remodeling processes such as histone ubiquitination or methylation appeared positively regulated in the different tissues, which may also impact gene transcription (fig. IV.14-25). Regulators of chromatin compaction, like the transcription factors described above, can influence the transcription of metabolic genes. For instance, exercise increased the expression of histone demethylase KDM5A-encoding gene (lysine (K)-specific demethylase $5 \mathrm{~A}$ ), which plays a role in the regulation of oxidation-reduction in mitochondria as direct repressor of metabolic regulatory genes (table IV.3; Váraljai et al., 2015). Interestingly, PGC-1a is also a coactivator of KDM5A target genes. Conversely, metabolites from the mitochondrial oxidation-reduction activity can regulate gene transcription. For example, Tet1 and Tet2 (tet methylcytosine dioxygenase 1 and 2) expression was reduced in the 


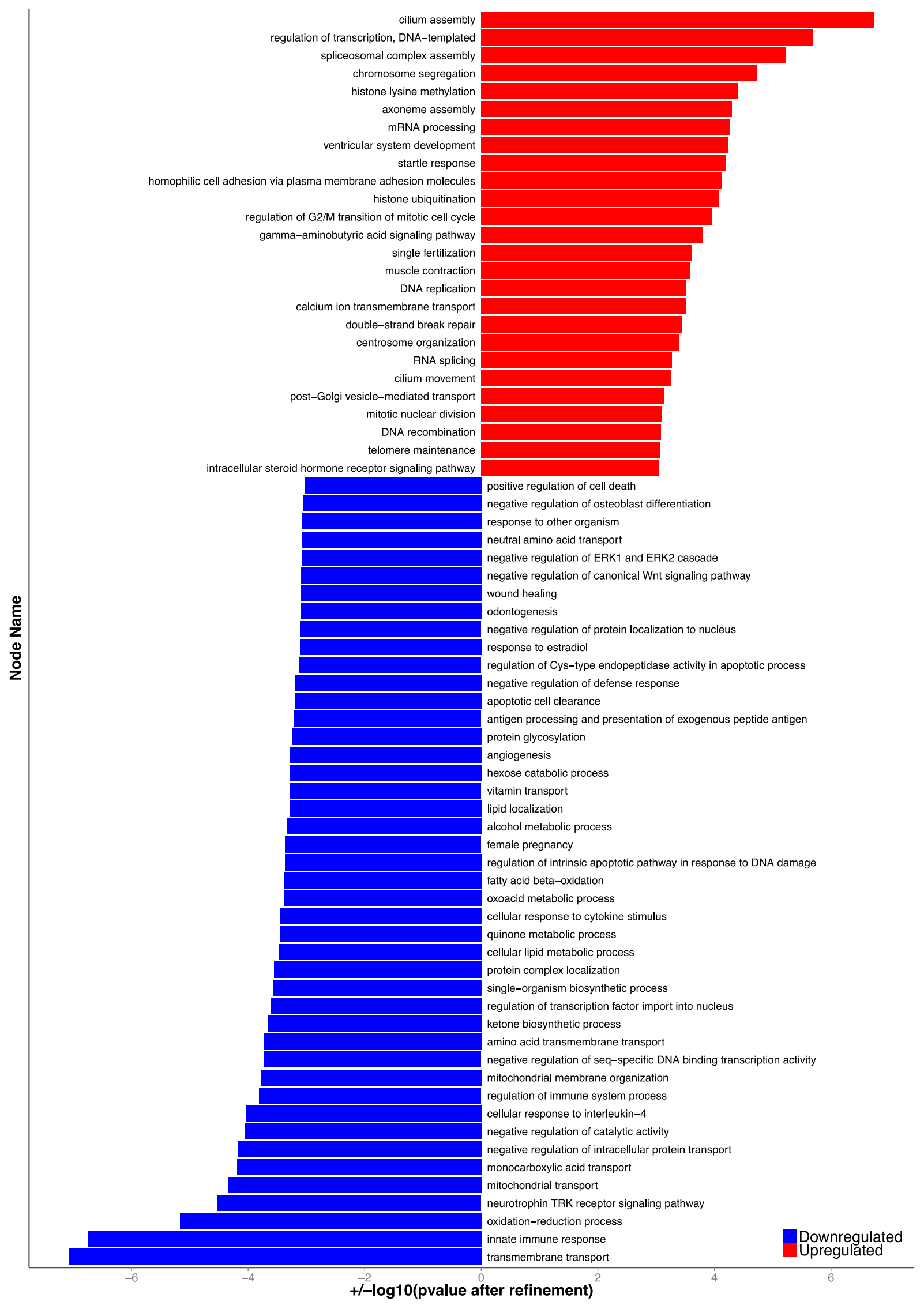

Figure IV.18. Biological processes activated or repressed upon exercise training in the dorsal spinal cord. (GO terms enrichment after computational functional analysis; $p$-value after refinement $<0.5)$ 


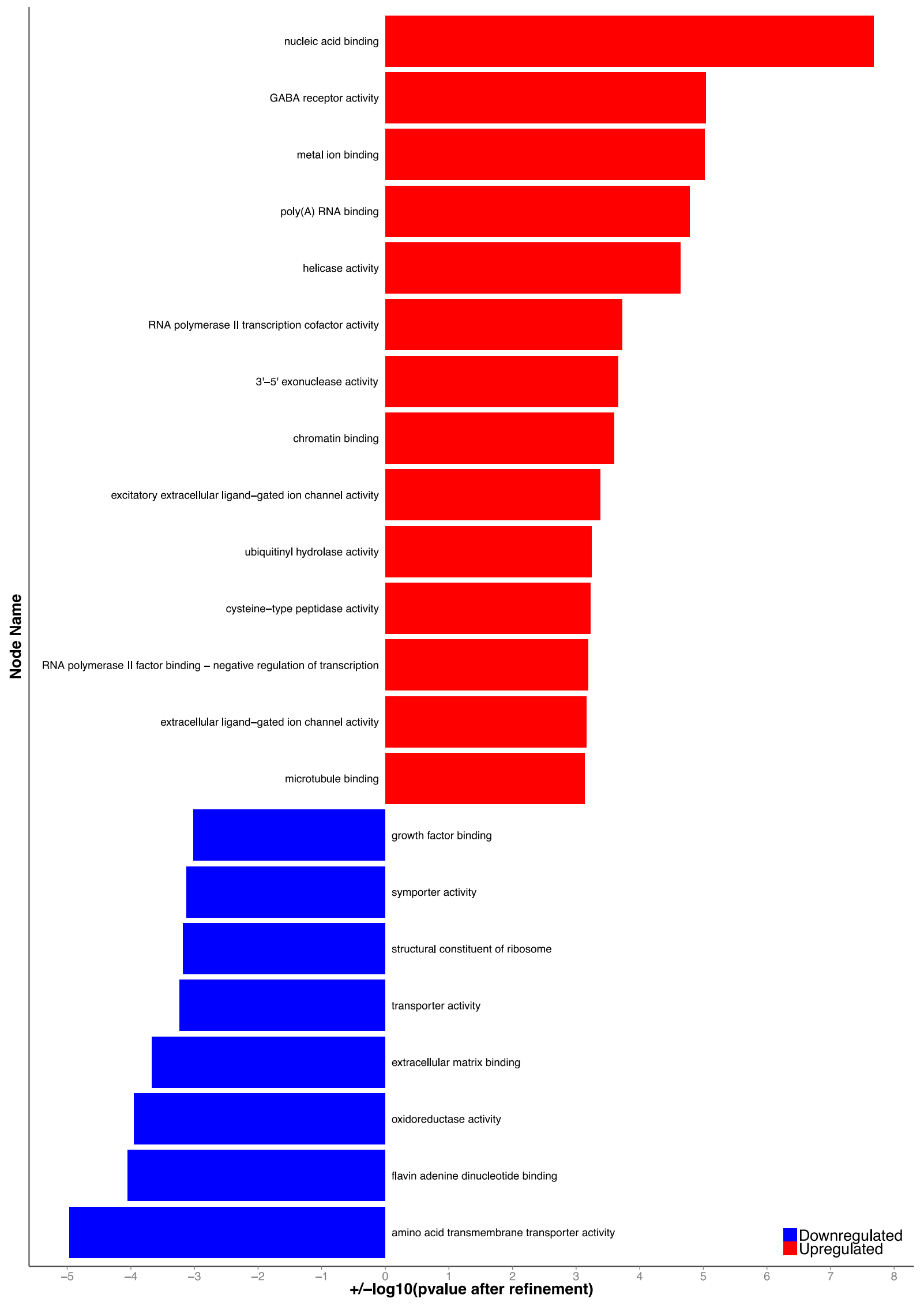

Figure IV.19. Molecular functions activated or repressed upon exercise training in the dorsal spinal cord. (GO terms enrichment after computational functional analysis; $p$-value after refinement $<0.5)$ 
A

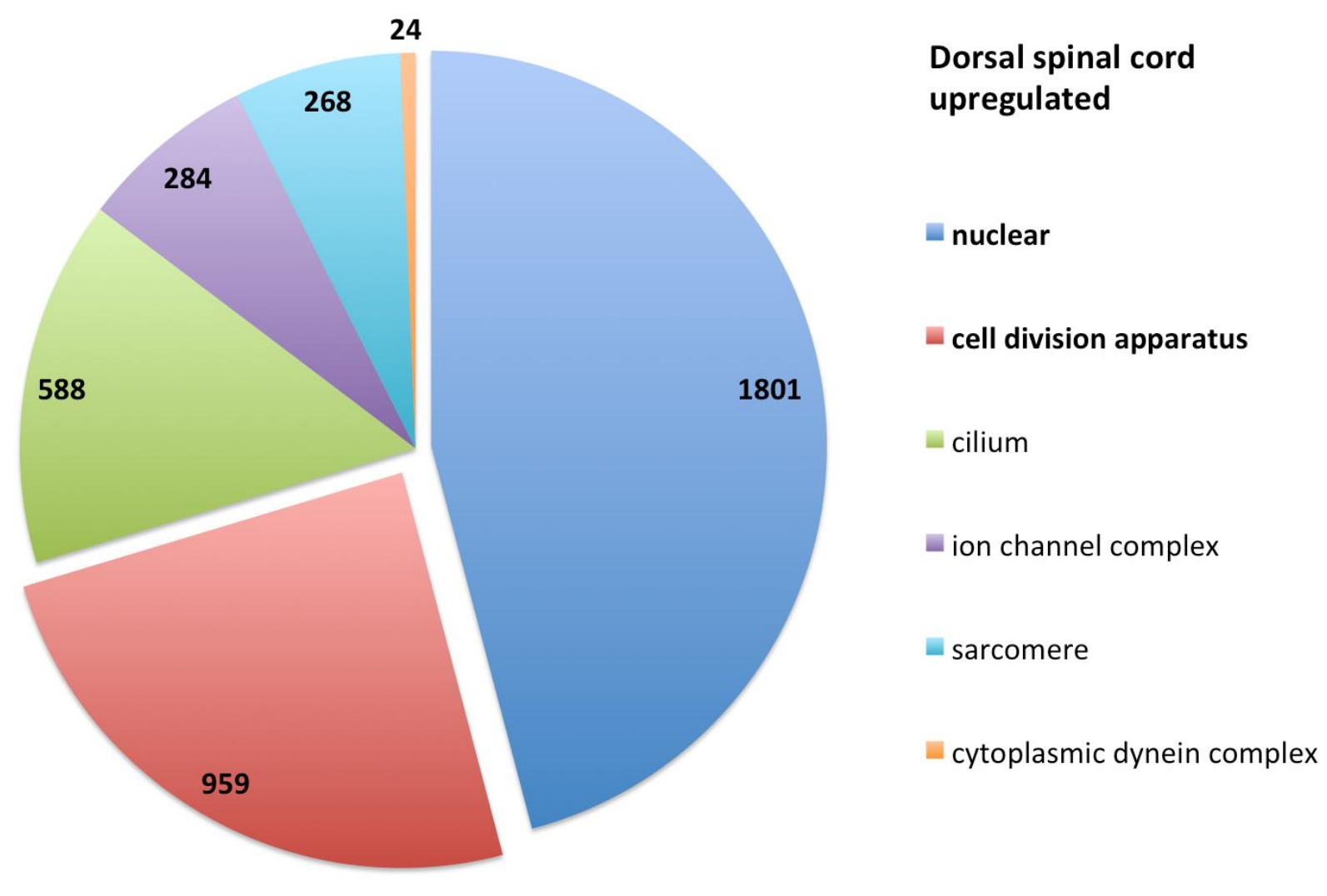

B

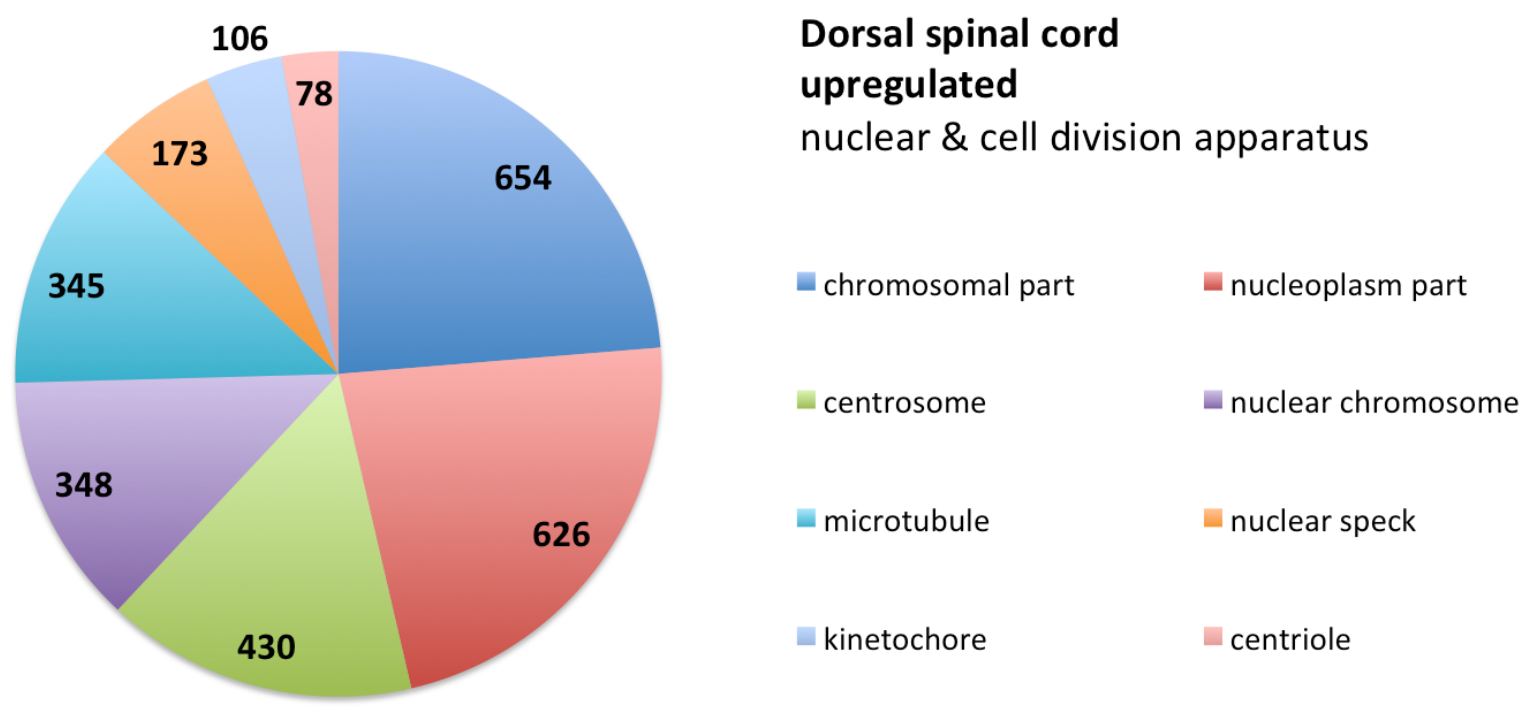

Figure IV.20. Exercise training mainly activated the nuclear compartment in the dorsal spinal cord. A. Principal cellular components which activity is enhanced upon exercise. B. Detail of the components in the nucleus and cell division apparatus. (GO terms enrichment after computational functional analysis; $p$-value after refinement $<0.5$; number of genes expressed in dorsal spinal cord, which product localizes in the compartment) 
A

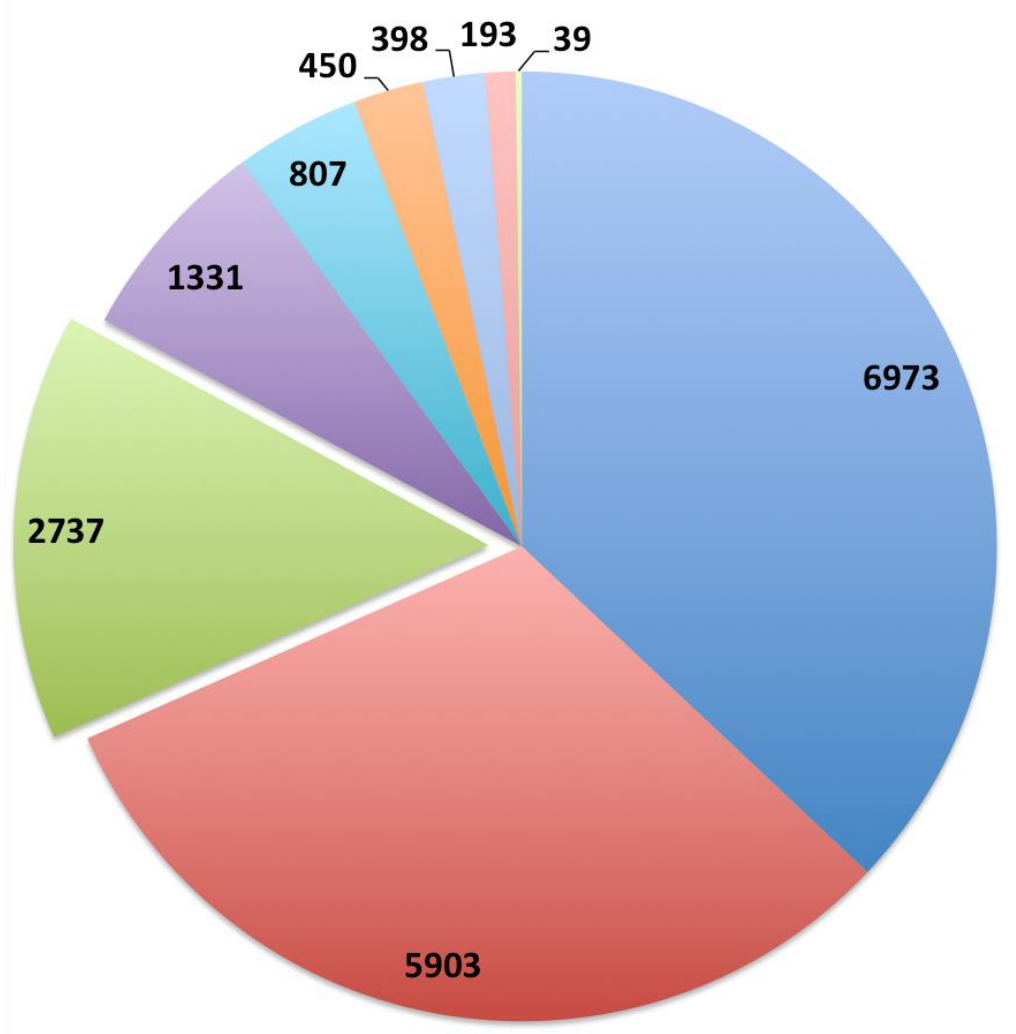

\section{Dorsal spinal cord downregulated}

vesicle

- integral component of membrane

mitochondrion

cytosol

endoplasmic reticulum membrane

Golgi membrane

proteinaceous extracellular matrix

- integral component of organelle membrane

autophagic vacuole

B

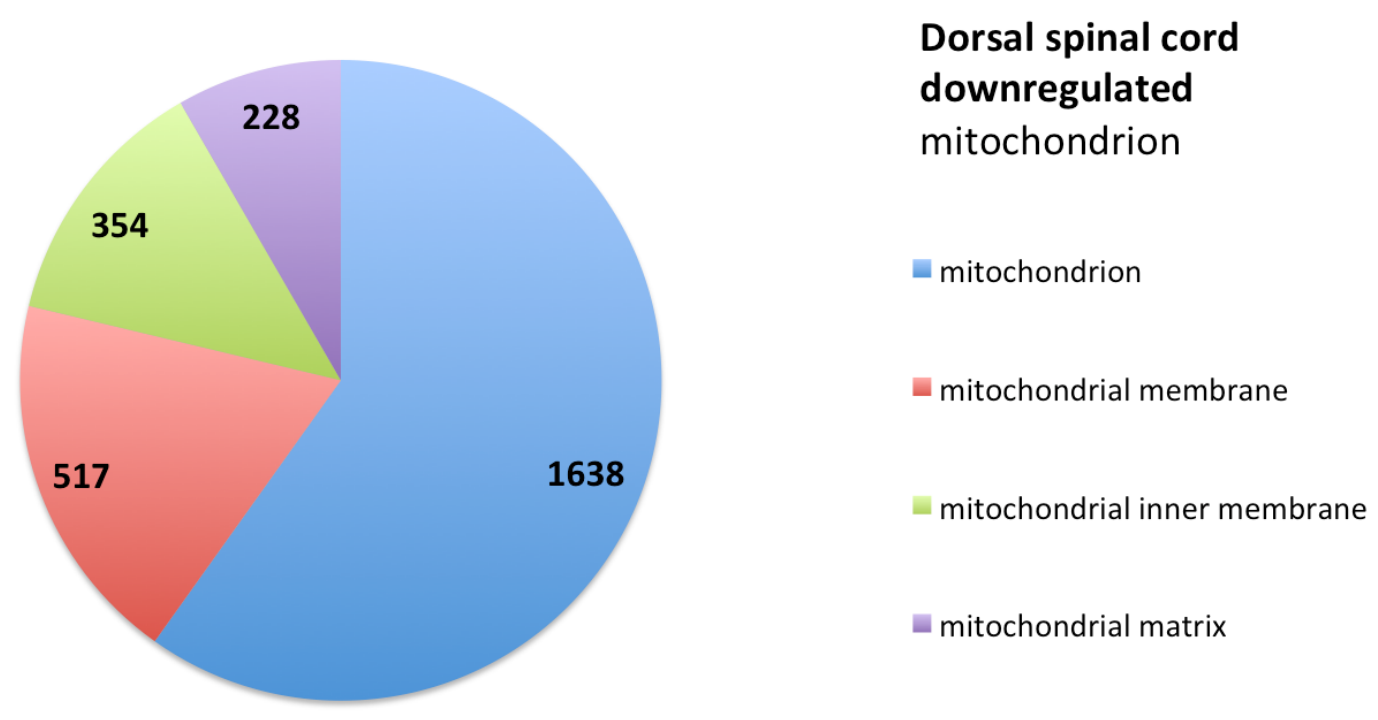

Figure IV.21. Exercise training mainly repressed activity of the mitochondrial compartment in the dorsal horn of the spinal cord. A. Principal cellular components which activity is repressed upon exercise. B. Detail of the mitochondrial sub-compartments. (GO terms enrichment after computational functional analysis; $p$-value after refinement $<0.5$; number of genes expressed in dorsal spinal cord, which product localizes in the compartment) 
experiment (table IV.3). TET1 and TET2 are major enzymes of DNA and histone demethylation, which activity is regulated by the metabolites in the Krebs cycle (Salminen et al., 2014). Interestingly, exercise appeared to also modify mitochondrial functions, which could be linked to the changes described above.

Firstly, the down-regulation of the mitochondrial membrane organization suggests an alteration of the fission-fusion processes and/or mitochondrial transmembrane protein transport (fig. IV.26). The balance between mitochondrial fusion and fission involves YME1L1 (YME1-like 1 ATPase), which gene expression was up-regulated after exercise (table IV.3; Anand et al., 2014). In contrast, the expression of the mitochondrial fusion factor Mitofusin 2-encoding gene (Mfn2), which participates in mitochondrial fusion, and MUL1-encoding gene (mitochondrial E3 ubiquitin protein ligase 1), which promotes mitochondrial fragmentation were both down-regulated (table IV.3; Bach et al., 2003; Lokireddy et al., 2012).

Secondly, several lines of evidence support a repression of the mitochondrial respiratory chain activity (hexose catabolic process, fatty acid $\beta$-oxidation, oxidationreduction process) (fig. IV.26). Interestingly, the repression of Mitofusin 2 notably reduces glucose oxidation and cell respiration (Bach et al., 2003). In addition, the expression of the gene encoding BCKDHA (branched chain ketoacid dehydrogenase $E 1 \alpha)$, a mitochondrial enzyme involved in the metabolism of the indispensable amino acids leucine, isoleucine and valine, was down-regulated (table IV.3; Hatzawa et al., 2014). Branched-chain amino acid metabolism may also be impacted by the reduced expression of Ethe1 (ethylmalonic encephalopathy 1), which product is implicated in mitochondrial oxidative phosphorylation (table IV.3; Hildebrant et al., 2013; Parikh et al., 2008). A third down-regulated gene, Mlycd (malonyl-CoA decarboxylase), is involved in fatty-acid oxidation (table IV.3; Saha and Ruderman, 2003). Besides, an increase in the gene expression of the mitochondrial reductase RRM2B (ribonucleotide reductase $\mathrm{M} 2 \mathrm{~B}$ ) was observed. RRM2B is the rate-limiting enzyme in de novo dNTP (deoxyribonucleoside triphosphate) synthesis required for mtDNA replication and repair (table IV.3; Pontarin et al., 2012). Further, the down-regulation of co-annotated processes such as the generation of precursor metabolites and energy, the hydrogen ion transmembrane transport or the cytochrome-c activity, support these observations (fig. IV.14-25).

Finally, the decrease in function of structural constituent of the ribosome indicates a reduction of the endoplasmic reticulum activity (fig. IV.26). Interestingly, 


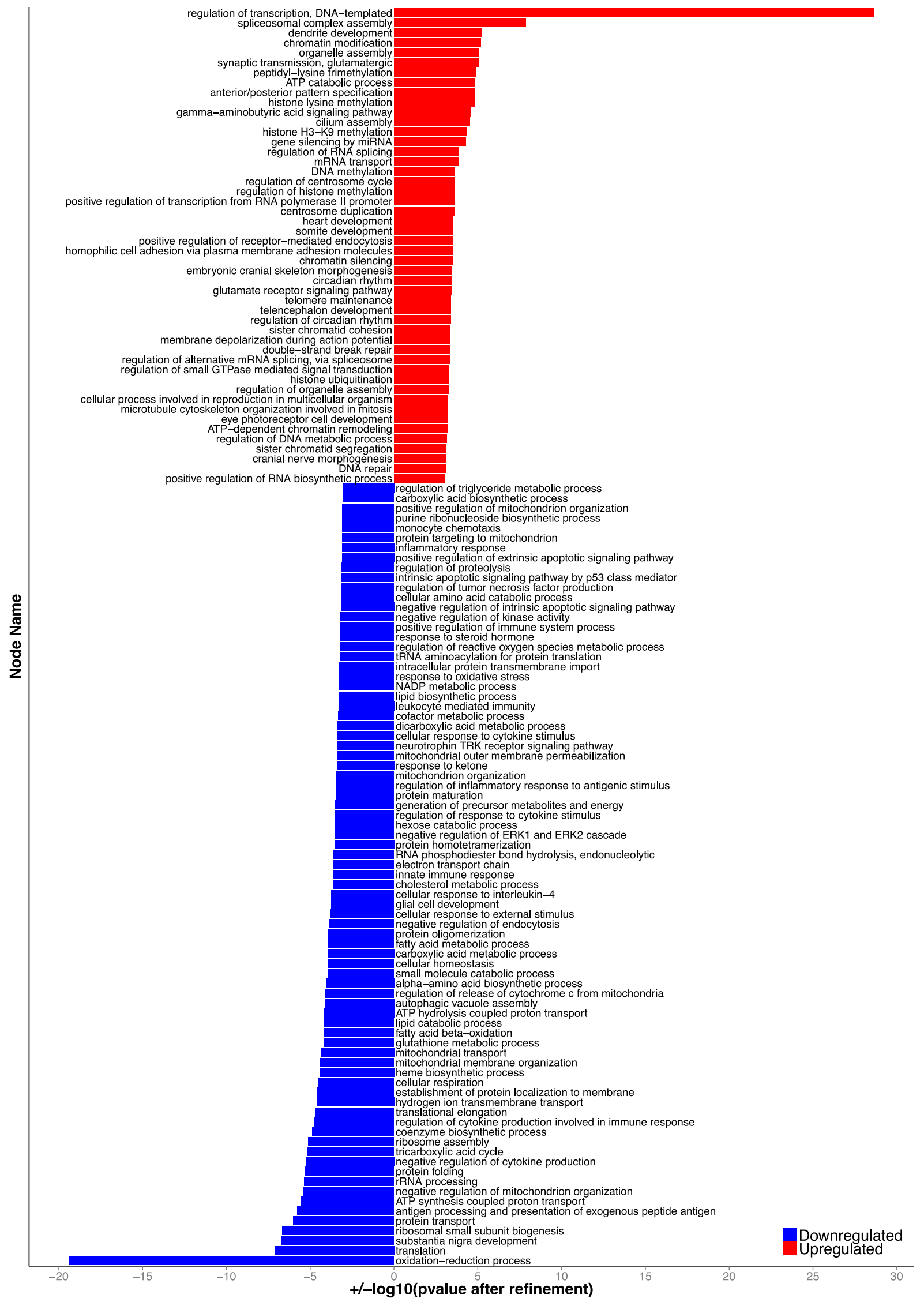

Figure IV.22. Biological processes activated or repressed upon exercise training in the ventral spinal cord. (GO terms enrichment after computational functional analysis; $p$-value after refinement $<0.5)$ 


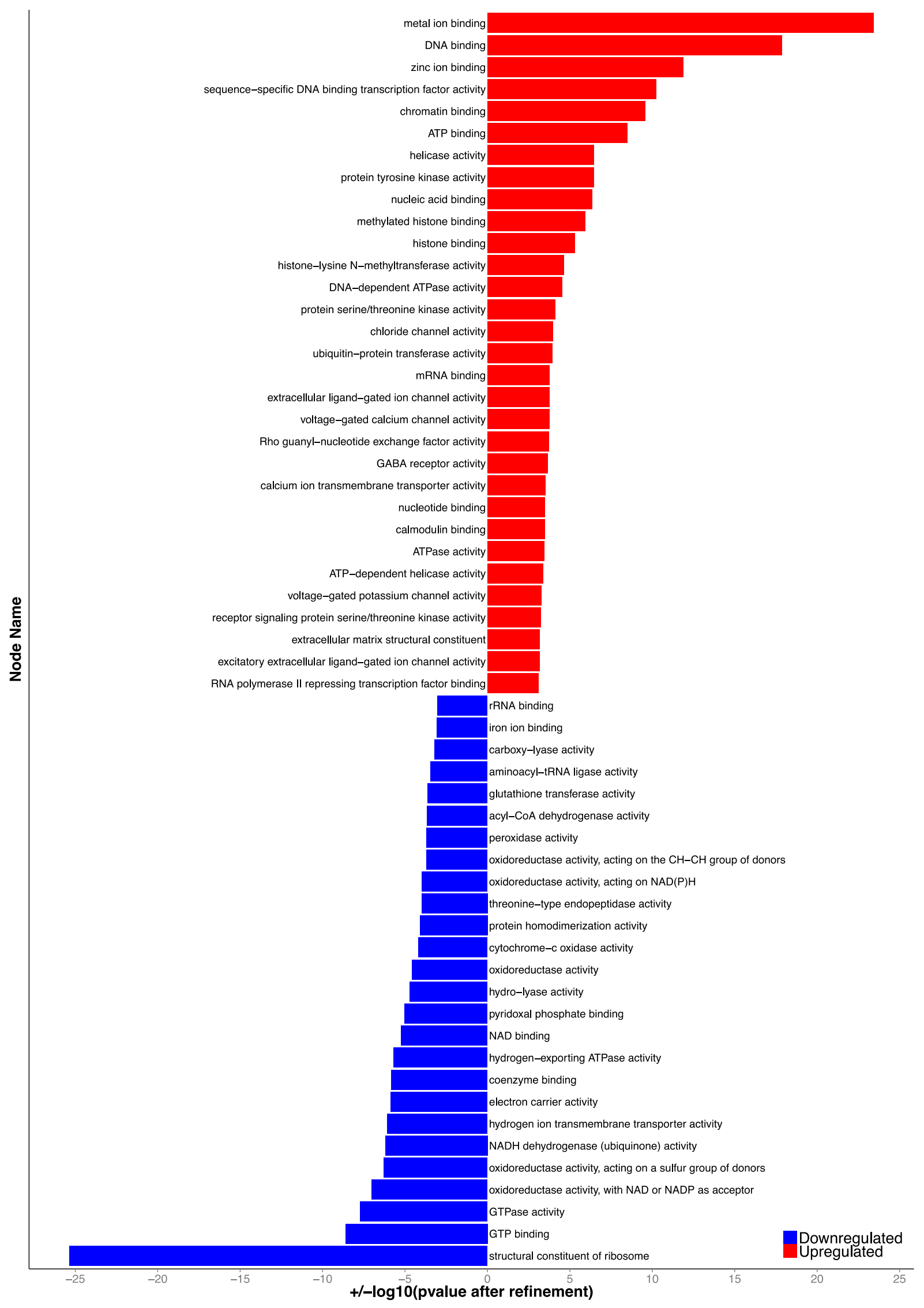

Figure IV.23. Molecular functions activated or repressed upon exercise training in the ventral spinal cord. (GO terms enrichment after computational functional analysis; $p$-value after refinement $<0.5)$ 
A

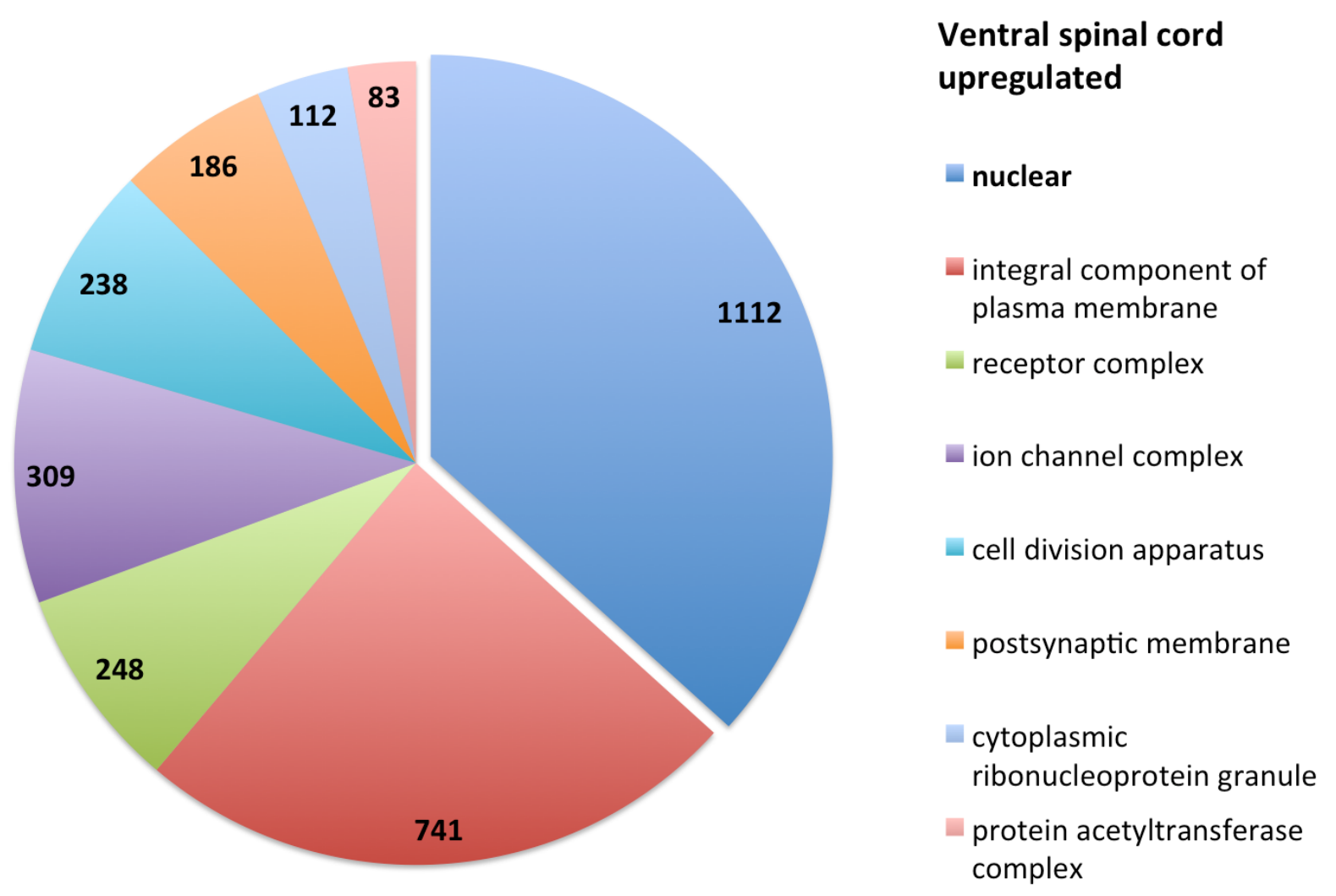

B

31

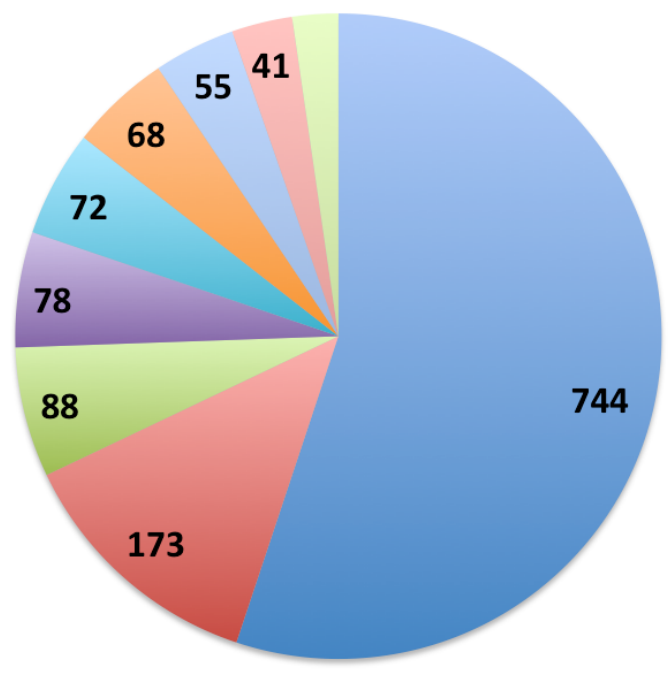

Ventral spinal cord upregulated

nuclear \& cell division apparatus

$$
\begin{aligned}
& \text { chromosome } \\
& \text { nuclear speck } \\
& \text { PML body } \\
& \text { - centriole } \\
& \text { ciliary basal body } \\
& \text { - histone methyltransferase complex } \\
& \text { chromosome, telomeric region } \\
& - \text { PcG protein complex } \\
& \text { nuclear heterochromatin }
\end{aligned}
$$

Figure IV.24. Exercise training mainly activated the nuclear compartment in the dorsal spinal cord. A. Principal cellular components which activity is enhanced upon exercise. B. Detail of the components in the nucleus and cell division apparatus. (GO terms enrichment after computational functional analysis; $p$-value after refinement $<0.5$; number of genes expressed in ventral spinal cord, which product localizes in the compartment) 


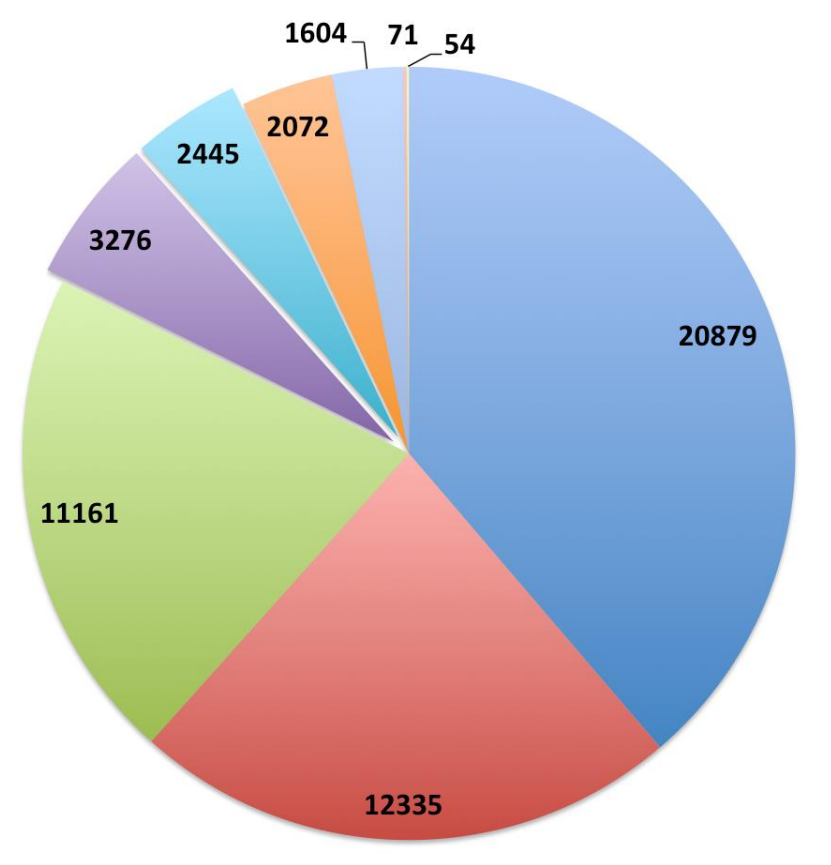

\author{
Ventral spinal cord \\ downregulated \\ organelle \\ - intracellular \\ cytosol \\ mitochondrion \\ endoplasmic reticulum \\ extracellular vesicular \\ exosome \\ intracellular vesicle \\ oxidoreductase complex \\ proton-transporting ATPase \\ complex
}

A

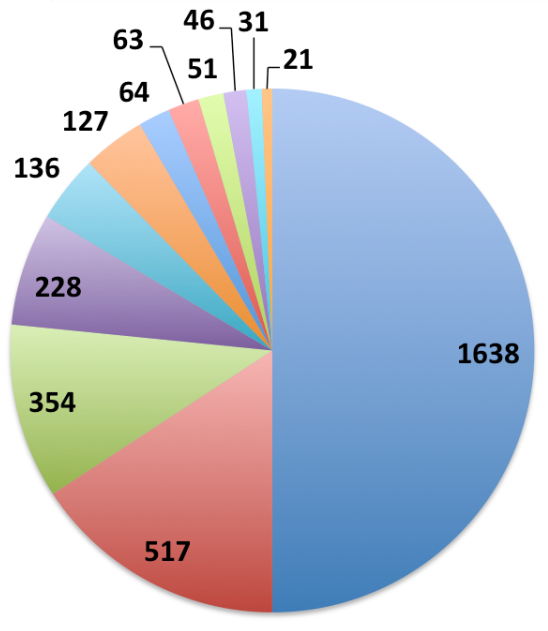

Ventral spinal cord downregulated mitochondrion

B

mitochondrion

mitochondrial membrane

- mitochondrial inner membrane

- mitochondrial matrix

- mitochondrial membrane part

mitochondrial outer membrane

respiratory chain

mitochondrial intermembrane space

mitochondrial respiratory chain

mitochondrial ribosome

mitochondrial respiratory chain complex I

mitochondrial proton-transporting ATP synthase complex

66

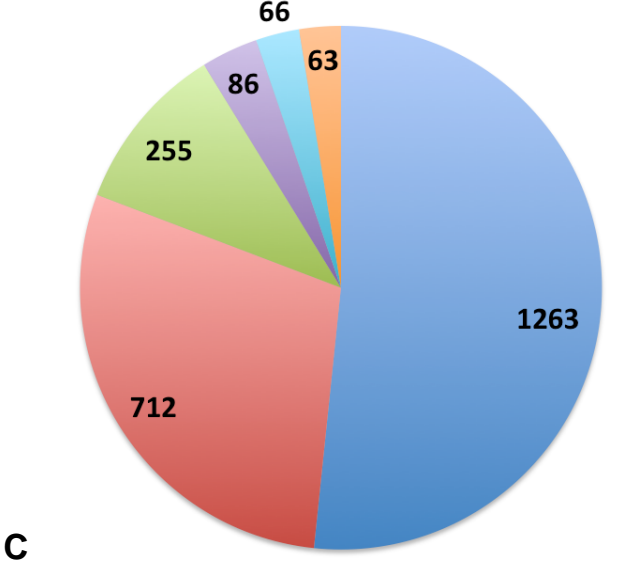

Ventral spinal cord downregulated endoplasmic reticulum

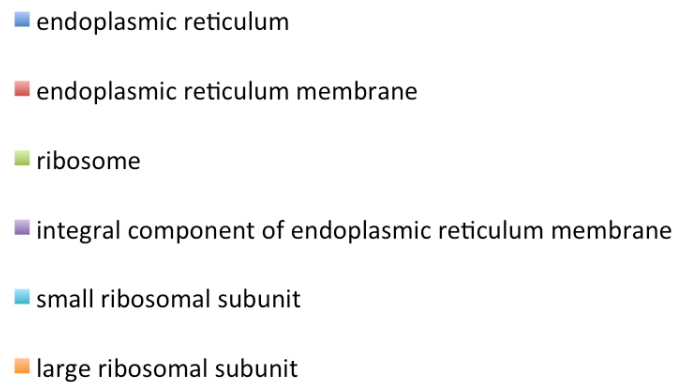

endoplasmic reticulum

Endoplasmic reticulum membrane

ribosome

- integral component of endoplasmic reticulum membrane

small ribosomal subunit

large ribosomal subunit

Figure IV.25. Exercise training mainly repressed activity of the mitochondrial and endoplasmic reticulum compartments in the dorsal spinal cord. A. Principal cellular components which activity is repressed upon exercise. B. Detail of the mitochondrial compartments. C. Detail of the endoplasmic reticulum compartments. (GO terms enrichment after computational functional analysis; $p$-value after refinement $<0.5$; number of genes expressed in ventral spinal cord, which product localizes in the compartment) 
BCKDHA appears to be also important for protein synthesis and ETHE1 may have implications in post-translational modifications (Hatzawa et al., 2014; Hildebrant et al., 2013). Moreover, the expression of Rps4I (ribosomal protein S4-like) and Rpl29 (ribosomal protein L29) was decreased following training (fig. IV.26). RSP4L is possibility involved in selective mRNA recognition and translation, and the deletion Rp/29 is accompanied by abnormal assembly of the two ribosomal subunits, which decreases the translational capacity of cells and thus reduces protein biosynthesis rates (table IV.3; Sugihara et al., 2013; Kirn-Safran et al., 2007). This is further supported by the down-regulation of translation and translation elongation, rRNA processing or ribosomal complex assembly in individual tissues (fig. IV.14-25).

Taken together these results indicate a common adaptive response of the different components of the neuromuscular system to physical exercise. On one hand, the expression regulation of genes involved in the regulation of chromatin modification, spliceosomal complex assembly and transcription, suggest an enhanced activity in the nucleus. On the other hand, the expression regulation of genes that play a role in mitochondrial biogenesis and localization, or implicated in ribosomal complex assembly and the regulation of translation suggest a repression of the activity in mitochondria and the endoplasmic reticulum. Moreover, many of the pathways that appear to change upon exercise are interconnected, with certain genes being shared by more than one pathway or cellular process. In particular, the nucleus and the mitochondrion appear to undergo changes that can affect each other. This suggests a synergistic adaptation of the different cellular compartment organization and function.

\subsubsection{Transcriptional changes in the soleus}

In order to address exercise-induced adaptation of the muscle more specifically, alterations in gene expression levels that could support the adaptations observed by immunohistochemistry were investigated. The Myh7 and Myh2 genes, encoding the slow isoforms $\mathrm{MyHC} \mathrm{I}$ and MyHC Ila, respectively, exhibited high expression levels, thus reflecting the predominance of type I and Ila fibers shown by immunohistochemistry (see fig. IV.2 and table IV.1), whereas Myh1 and Myh4 coding for the fastest myosins MyHC IIx and MyHC IIb respectively, had comparatively low expression levels (fig. IV.27A). In addition, the slight shift towards "slower" fiber types 
B

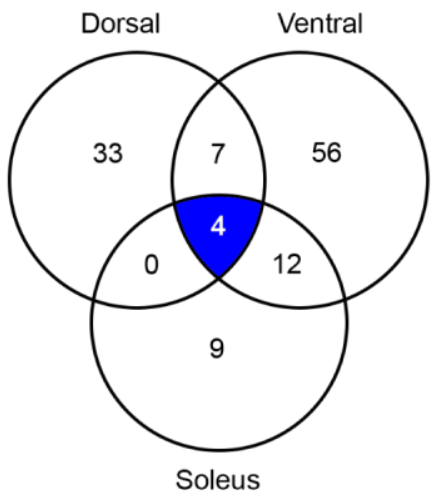

Soleus

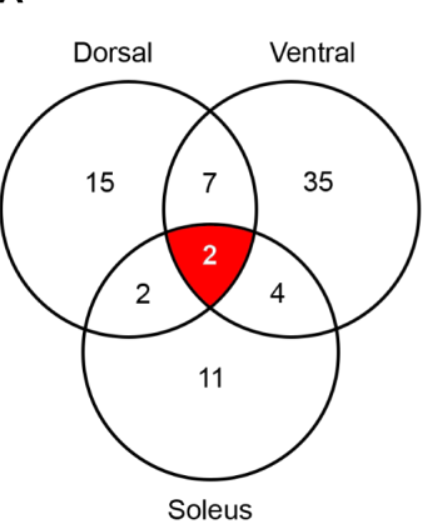

E
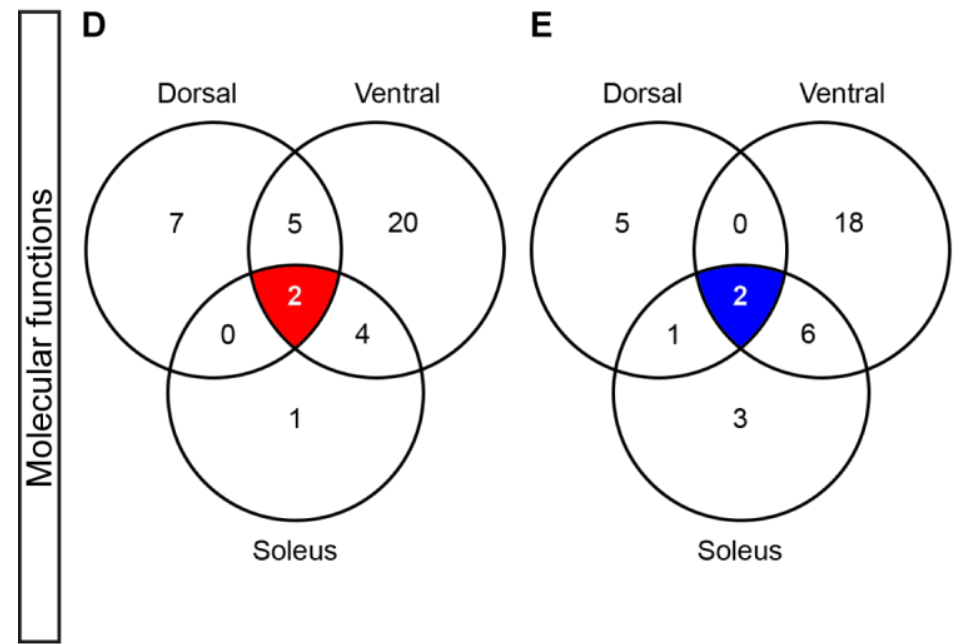

H
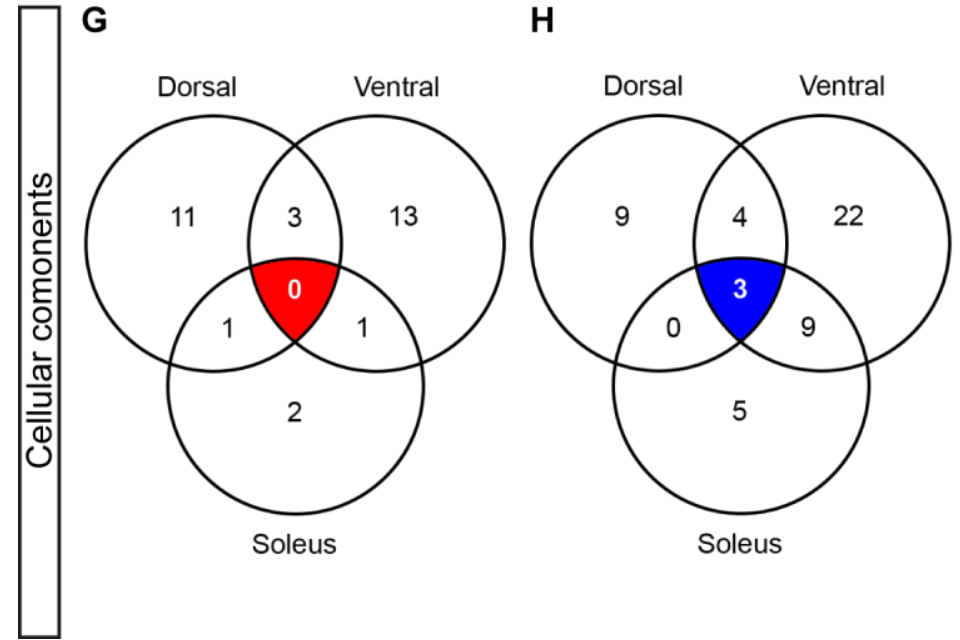

common enriched GO terms
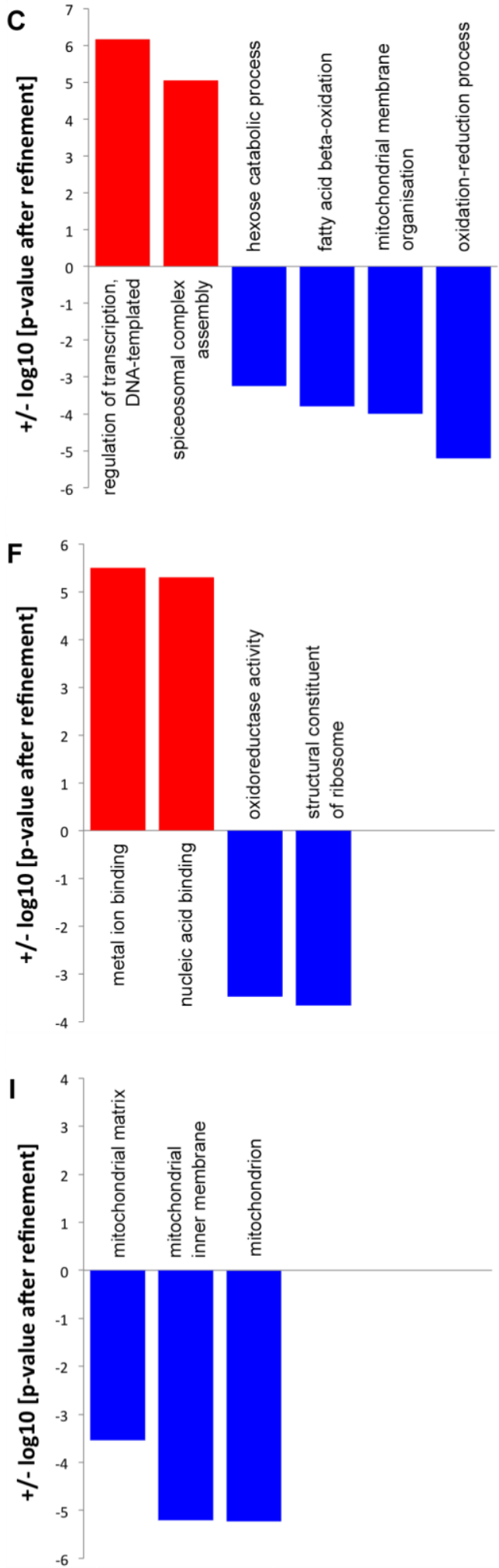

Figure IV.26. Common response of dorsal spinal cord, ventral spinal cord and soleus muscle to long-term endurance training. Venn diagrams represent in the three tissues the number of GO terms identified by computational functional analysis, which have a p-value after refinement $<0.5$ (A-B, D-E, G-H). Some biological processes (A-B), molecular functions (D-E) and cellular components (G-H) were similarly up- or down-regulated after exercise in all three tissues. The details of the are shown in the bargraphs (C, F, I). Globally, the mitochondrion compartment and function is down-regulated, whereas nucleic acid regulation is activated. (color code: blue $=$ repression, red $=$ activation) 


\begin{tabular}{|c|c|c|}
\hline Srsf1 & 1.15 & spliceosomal complex assembly; nucleic acid binding \\
\hline Ptbp2 & 1.16 & spliceosomal complex assembly; nucleic acid binding \\
\hline Psip1 & 1.19 & spliceosomal complex assembly; nucleic acid binding \\
\hline Akt1 & 1.15 & regulation of transcription, DNA-templated; oxidation-reduction process; fatty acid beta-oxidation; mitochondrion \\
\hline Pten & 1.20 & regulation of transcription, DNA-templated; metal ion binding; mitochondrion \\
\hline lgf1 & 1.22 & regulation of transcription, DNA-templated; oxidation-reduction process \\
\hline Esrra & 1.19 & regulation of transcription, DNA-templated; nucleic acid binding; metal ion binding \\
\hline Hdac5 & 1.16 & regulation of transcription, DNA-templated; nucleic acid binding \\
\hline Mef2a & 1.22 & regulation of transcription, DNA-templated; nucleic acid binding \\
\hline Kdm5a & 1.12 & oxidation-reduction process; oxidoreductase activity; nucleic acid binding; metal ion binding \\
\hline Tet2 & 1.28 & regulation of transcription, DNA-templated; nucleic acid binding; metal ion binding; \\
\hline Tet1 & 1.23 & \& oxidation-reduction process; oxidoreductase activity \\
\hline Mul1 & 1.14 & mitochondrial membrane organization; mitochondrion; metal ion binding \\
\hline Mfn2 & 1.08 & mitochondrial membrane organization; mitochondrion \\
\hline Yme1/1 & 1.16 & mitochondrion; mitochondrial inner membrane; metal ion binding \\
\hline Bckdha & 1.17 & oxidation-reduction process; oxidoreductase activity; mitochondrion \\
\hline Ethe1 & 1.15 & oxidation-reduction process; oxidoreductase activity; mitochondrion; mitochondrial matrix; metal ion binding \\
\hline Mlycd & 1.13 & oxidation-reduction process; fatty acid beta-oxidation; mitochondrion; mitochondrial matrix \\
\hline Rrm $2 b$ & 1.22 & oxidation-reduction process; oxidoreductase activity; mitochondrion; metal ion binding \\
\hline Rps4l & 1.27 & structural constituent of ribosome; nucleic acid binding \\
\hline Rp/29 & 1.17 & structural constituent of ribosome \\
\hline
\end{tabular}

Table IV.3. Selected genes from the common response to training, which play a role in the regulation of transcription and splicing, in oxidation-reduction processes and in mitochondrial membrane organization. The GO terms of the three taxa (biological processes, molecular functions and cellular components) similarly regulated in the three tissues, show some overlap with regard to the genes involved. The average fold-changes indicate that the rate of change in expression level of the selected genes is limited, although the change is statistically significant. GO terms enrichment after computational functional analysis; $p$-value after refinement $<0.5$; fold-change calculated as average of fold-change in the three tissues; color code: blue = down-regulated expression level, red = up-regulated expression level) 
observed by immunohistochemistry in soleus muscles of trained mice (see fig. IV.2 and table IV.1) was reflected by corresponding changes in gene expression detected by RNA sequencing (fig. IV.27). Expression level of $M y h 7$ was significantly increased ( $p$-value $<0.001$, run/ctrl fold-change $=1.36$ ), while the expression of Myh2 was decreased ( $p$-value $=0.035$, run/ctrl fold-change $=0.87$ ), thus reflecting the increased slow type I and reciprocally decreased fast types Ila and IIx in soleus (table IV.1). The expression of Myh1 and Myh4 was unaffected by exercise. Moreover, endurance training increased the expression of Prox1 (prospero homeobox 1 ) that is specifically implicated in slow fiber-type specification and whose loss promotes a shift towards fast fibers (fig. IV.27B; Petchey et al., 2014). These results indicate that although extensive fiber-type transition was limited when looking at the histological results, the transcriptome data revealed a comprehensive shift towards a slow fiber type gene expression signature.

\subsubsection{Transcriptional changes specific to the neuromuscular synapse}

Transcriptional changes in the soleus muscle and ventral spinal cord were investigated in order to identify specific adaptation of the NMJ that could underpin changes in pre- and post-synaptic NMJ observed by immunohistochemistry. The results of the histological analysis of NMJs in sedentary and trained animals suggest that soleus NMJs adapted through synaptic expansion paralleled by an increase in mitochondrial volume, whereas the surface covered by TA NMJs underwent contraction, associated with enhanced interconnectivity of the mitochondrial network (see fig. IV.4-11). The data also suggest that these changes may not merely reflect pre-synaptic arrangements accompanying the change in post-synapse size, but rather specific modifications elicited in MNs in response to increased activity. The RNA sequencing results in the dorsal and ventral spinal cord, as well as in soleus muscle showed that expression levels of genes implicated in synapse remodeling and synaptic transmission were altered by 12 weeks of voluntary wheel-running (fig. IV.28).

Two genes from the common response to exercise presented in table 3 are involved in the regulation of synaptic genes. The splicing regulator PTBP2 (polypyrimidine tract binding protein 2) is implicated in regulating pre- and postsynaptic assembly and synaptic transmission, while MEF2 is implicated in regulating 
A

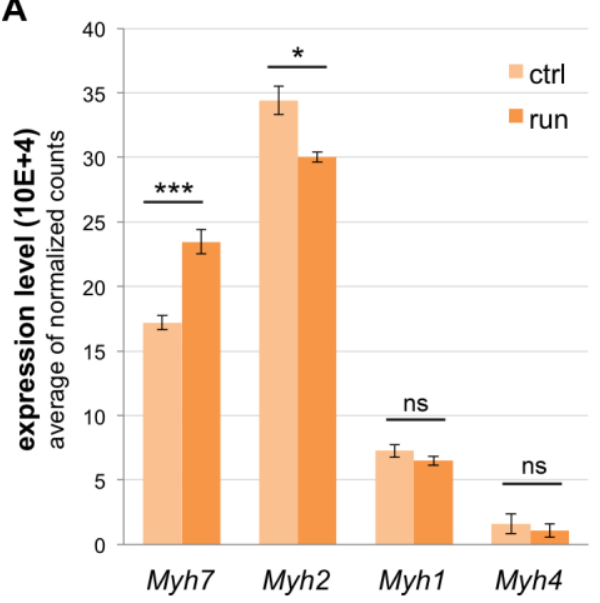

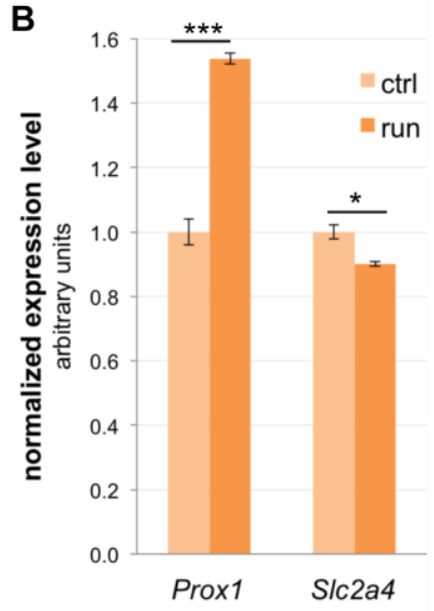

Figure IV.27. Twelve weeks wheel running induces changes in gene expression in the soleus muscle. A. Expression level of the Myh genes encoding the four adult skeletal muscle MyHC isoforms Myh7 (MyHC I), Myh2 (MyHC Ila), Myh1 (MyHC IIx) and Myh4 (MyHC Ilb) measured by RNA sequencing. Soleus predominantly expresses the slow Myh7 and the fast resistant Myh2 isoforms. Exercise induces the expression of Myh7 and reduces the expression of Myh2. B. Endurance training increases the expression of Prox 1 that is specifically implicated in slow-twitch fiber-type specification. The expression of S/c2a4, which encodes the GLUT4 glucose transporter protein implicated in insulin sensitivity, is down-regulated. (significance: ${ }^{*} p$-value $<0.05$, ${ }^{* *} p$-value $<0.01$, ${ }^{* * *} p$-value $<0.001$, ns: no significance; error bars: $\mathrm{Cl}=0.95$; for each paradigm $\mathrm{n}=6 \mathrm{C} 57 \mathrm{BL} / 6 \mathrm{~J}$ animals)

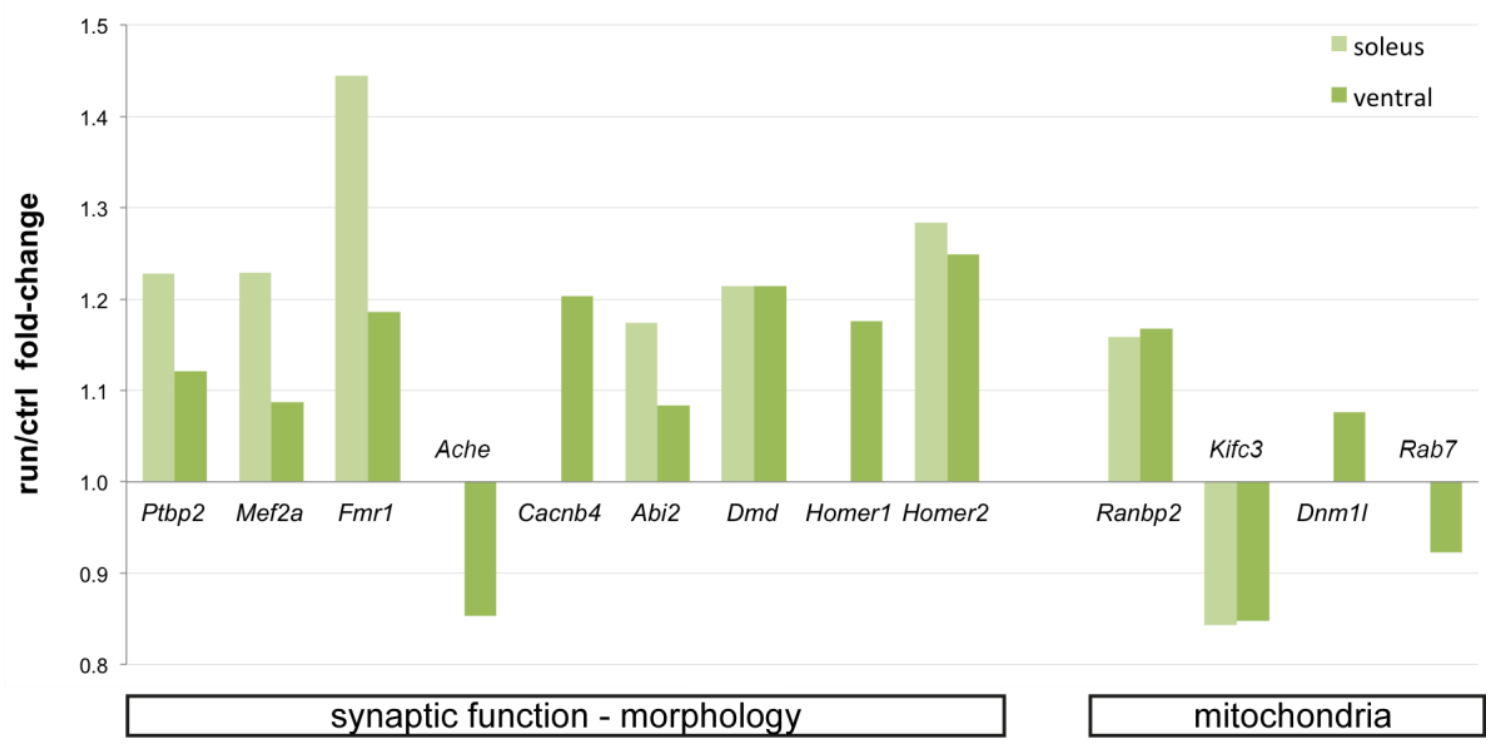

Figure IV.28. Endurance training regulates the expression level of genes involved in synaptic processes: transmission, morphogenesis and mitochondrial organization. The expression level of the selected genes in soleus and ventral spinal cord is expressed as fold-change in the running samples compared to control samples. The products of the selected genes play different roles in preand post-synapse maintenance and signal transduction. Some of the gene products are involved in mitochondrion motility, distribution or fission-fusion dynamics. The regulation of the gene expression may impact NMJ structure and function to adapt to elevated physical activity. (gene selection criterion: p-value $<0.5)$ 
the transcription of synaptic genes and also plays a key role in post-synaptic maturation that condition accurate pre-synaptic active zone localization (see table IV.3; fig. IV.28; Zheng et al., 2012; Flavell et al., 2008; Prokop et al., 1996). In addition, MEF2 also functions together with FMR1 (fragile $X$ mental retardation syndrome 1) to eliminate excitatory synapses during brain development (Pfeiffer et al., 2010). Expression of Fmr1 was increased after exercise. FMR1 is also implicated in presynaptic long-term potentiation in cortical neurons, as well as in an RNA-editing pathway that ensures proper NMJ synaptic architecture (Bhogal et al., 2011; Koga et al., 2015). Interestingly, the expression of the ACHE-encoding gene (acetylcholinesterase), which deletion causes smaller nerve terminals and diminished pre- and post-synaptic surface contacts, was reduced (fig. IV.28; Adler et al., 2011). Ache as well as Cacnb4 (calcium channel, voltage-dependent, $\beta 4$ subunit) null mutants, display defects in synaptic transmission. Cacnb4, up-regulated after exercise, encodes a regulatory subunit of the voltage-gated calcium channel, which association with the $\alpha_{1 \mathrm{~A}}$ subunit increases current amplitude at the motor endplate (fig. IV.28; Arikkath and Campbell, 2003). CACNB4 also serves to communicate the activity of calcium channels to the nucleus through its nuclear targeting, and possibly play a role as scaffolding protein that allows specific downstream effects (Subramanyam et al., 2010; Weiss, 2006). Moreover, physical training enhanced the expression of genes encoding proteins specifically involved in synapse assembly, remodeling and maintenance. For instance, ABI2 (abl-interactor 2) regulates actin structure and presynaptic axon guidance, and is a critical mediator of post-synaptic assembly and remodeling at the NMJ (fig. IV.28; Finn et al., 2003). Dystrophin (Dmd) is required for organizing large $A C h R$ aggregates on the post-synapse and the calcium sensors HOMER 1 and 2 (homer homolog 1 and 2) have been proposed to modulate the activity of various ion channels and of the transcription factor NFAT (nuclear factor of activated T cells), that modulates skeletal muscle differentiation (fig. IV.28; Kong and Anderson, 1999; Salanova et al., 2011).

Furthermore, exercise modulated the expression of genes implicated in mitochondrial network remodeling, which may account for a part of the adaptive mechanisms at the pre-synaptic NMJ. Notably, the products of the up-regulated Ranbp2 (RAN binding protein 2) and the down-regulated Kifc3 (kinesin family member C3) genes are regulators of mitochondria trafficking (fig. IV.28). RANBP2 modulates mitochondrial membrane potential and is specifically implicated in the 
kinesin-dependent transport of mitochondria, while KIFC3 is abundantly expressed at neuronal terminals and mediates minus-end directed (retrograde) transport for proper subcellular distribution of mitochondria (Cho et al., 2007; Hoang et al., 1999; Dietrich et al., 2013). In addition, training also mediated the regulation of genes implicated in mitochondrial fission-fusion processes such as Dnm1/ (dynamin 1-like, also known as Drp1) or Rab7 (RAB7, member RAS oncogene family) (fig. IV.28). The product of the up-regulated Dnm1/ gene contributes to mitochondrial division in mammalian cells, and is required for proper cellular distribution of mitochondria (Smirnova et al., 2001; Verstreken et al., 2005). More interestingly, DNM1L appears to be critical for the mobilization of reserve pool vesicles at the NMJ and its mutation severely affects neurotransmission (Verstreken et al., 2005). The RAB7 protein encoded by the down-regulated Rab7 gene is required for normal presynaptic growth and postsynaptic organization at the NMJ (Lee et al., 2013). Moreover, RAB7 is implicated in mitophagy that relies on the fusion of mitochondria with lysosomes (Yamano et al., 2014).

Taken together, these results indicate that specific synaptic gene expression changes are elicited in the soleus muscle and the ventral spinal cord in response to exercise. These genes are implicated in synaptic structure maintenance and synaptic transmission on one hand, and in mitochondrial morphology and subcellular distribution on the other hand. The regulation of these genes likely accounts for the observed adaptation of NMJ morphology and presynaptic mitochondrial networks. The regulation of mitochondrial biogenesis and dynamics may thus be tightly linked to the adaptation of NMJ structure and function to elevated physical activity. 


\section{Discussion}

Physical activity is of major importance in improving the quality of life. Exercise can have a considerable impact because it is relevant to the general "healthy" population and particularly interesting in preventing the development of pathologies and delaying the progression of diagnosed diseases. Unlike drugs, regular physical activity is accessible to anybody and does not require any prescription. However, the elaboration of an appropriate training is usually recommended when used as part of the treatment for a specific impairment. For example, exercise is used as adjunct in different cancer therapies, high intensity interval training is recommended to improve diabetes symptoms and interestingly, different forms of exercise promote MN survival, which delays the onset of motor deficits in ALS model mice (Brown at al., 2012; Bird and Hawley, 2012; Carreras et al., 2010; Deforges et al., 2009).

\subsection{Physical training - exercise paradigm}

Voluntary wheel running, the exercise paradigm used in this study, can be categorized as moderate endurance training, based on oxidative metabolism (aerobic). In contrast, strength or resistance training is based on a glycolytic (anaerobic) energy system. In muscle, both forms of exercise appear to lead to similar increases in mitochondrial abundance and improvements in glycemic control (Egan and Zierath, 2013). However, the intensity, frequency and duration of each exercise session imply distinct functional adaptations of the organism, with different impacts on the metabolic and molecular responses of any given tissue (Hildebrandt et al., 2003). It was shown that after 12 weeks of increased daily physical activity, physiological adaptations of muscle and cardiovascular systems have reached a steady-state level, and that biophysical properties of MNs have adapted (Beaumont and Gardiner 2003). Thus, the exercise-induced changes observed in this study are long-term stable adaptations of the different organs and tissues.

Besides, a common objection to the use of wheel running with laboratory animals is that this behavior is an artifact of captivity (Sherwin, 1998). However, a 
recent study showed that wild mice use running wheels that are placed in nature in a similar pattern compared to captive mice in cages equipped with identical running wheels (Meijer and Robbers, 2014). This suggests that wheel running is rather an elective than a stereotypic behavior. It is worth noting that distances run in wheels should not directly be compared with linear planar locomotion. All animals used in the present study were of C57BL/6J genetic background and had the same age at the start of the training. Moreover the MDO (Mito-Dendra2-flox;Olig2-Cre) mouse line is a reporter line and therefore MDO animals also present a wild-type phenotype. Since C57BL/6J and MDO males exhibited similar performances on average (data not shown), differences in performance in the selected animals were likely due to the exclusive use of C57BL/6J males, when both males and females MDO were used (see fig. 1). This observation is consistent with the previous demonstration that rat females run more than males (Eikelboom and Mills, 1988).

\subsection{Exercise-induced specific adaptations in muscles}

Alterations of the neuromuscular system upon long-term endurance training were first investigated in muscles. The fiber type distribution in soleus and plantaris suggest that the long but moderate intensity training induces a slight shift towards slower fiber types in slow as well as in fast muscles, in line with previously published data (see fig. 2 and 3; reviewed in Bassel-Duby and Olson, 2006, and Schiaffino et al., 2007). The rate of change appeared to be higher in soleus compared to plantaris, possibly due to the fact that only the slowest motor units, enriched in the soleus, are active during moderate intensity running (Zajac and Faden, 1985). The RNA sequencing experiment confirmed this slow-to-fast fiber-type transition in the soleus, where a slow phenotype was specifically promoted by enhancing Prox1 gene expression level (see fig. 29; Petchey et al., 2014). Surprisingly, endurance training induced the down-regulation of Slc2a4 encoding the GLUT4 glucose transporter that plays a role in muscle sensitivity to insulin (fig. 29). Previous studies reported transient Slc2a4 transcription after single bouts of exercise (Richter and Hargreaves, 2013). This suggests that chronic training and acute exercise differentially modulate glucose uptake in muscle. However, the present study did not investigate GLUT4 protein activity, which may be enhanced in a context of prolonged training. In 
addition, long-term endurance training increased Pten and decreased Akt1 gene expression in soleus as well as in the spinal cord (table 3). Since PTEN is an inhibitor of the PI3K/AKT/mTOR signaling pathway, both results indicate an inhibition of this pathway (Song at al., 2012). Resistance training, but not endurance training, activates PI3K/AKTmTOR signaling to induce skeletal muscle hypertrophy, and another study suggests that AKT1 signaling may function as a specific mediator of type II muscle hypertrophy (Bodine et al., 2001; Nader and Esser, 2001; Izumiya, 2008). Therefore, inhibition of AKT/mTOR pathway is consistent with the fast-to-slow muscle fiber transition induced by endurance training. However, wheel running induced an increase in lgf1 gene expression (table 3), consistently with another study, which found increased IGF-1 protein levels in the brain after moderate longterm exercise (Bayod et al., 2011). On the opposite of PTEN, IGF-1 activates $\mathrm{PI3K} / \mathrm{AKT} / \mathrm{mTOR}$ signaling pathway and promotes muscle growth (fig. 27; Schiaffino and Mammucari, 2011). Thus, in endurance training, IGF-1 may not be implicated in muscle growth but possibly exerts one or more of its numerous other roles, including the regulation of glucose uptake, inflammation processes, mitochondrial function or synaptic plasticity (Spielman et al., 2014).

\subsection{Neuromuscular system adaptation to training, the common changes}

The transcriptomic analysis showed that soleus gene expression profile was significantly altered after 12 weeks of voluntary wheel running (see fig. 13B, fig. 1417 and fig. 28). However, important changes in gene expression level also occurred in the ventral spinal cord and to a lesser extend in the dorsal spinal cord (see fig. 13A and fig. 18-25). Some of these regulations were common to all three tissues, whereas other changes were specific to either one of them. This suggests that exercise can elicit a common response from different organs and at the same time can induce tissue-specific changes in order to adapt individual organ properties and functions, and maintain the whole body homeostasis upon elevated activity. For instance, the circadian rhythms were positively regulated specifically in the ventral spinal cord (see fig. 22). Consistently, it was previously demonstrated that voluntary running entrains the circadian clock that can impact energy metabolism through the 
regulation of food intake or insulin sensitivity (Yamanaka et al., 2013; Marosi and Mattson 2014). Further, among the multiple biological processes affected by physical training in all three tissues, the cell cycle and cell division apparatus were positively regulated (see fig. 14,16,18, 20, 22 and 24). This could reflect the proliferation of satellite cells in the muscle, or the addition of myofiber nuclei in order to sustain increased protein synthesis (Bruusgaard et al., 2010). Although voluntary exercise was shown to promote both postnatal gliogenesis and neurogenesis in brain regions, these processes were never described in the spinal cord before (Cotman and Berchtold, 2002; Mandyam et al., 2007). Moreover, training increased cytokine production and release, and modulated the response to circulating cytokines and hormones, thereby regulating the communication between tissues (see fig. 14,18 and 22). However, in line with other studies, exercise suppressed inflammatory response that is usually associated with reduced physical activity (Handschin and Spiegelman, 2008). Strong evidence suggests that this is the mechanism by which physical activity prevents the development or ameliorates the symptoms of chronic diseases. Another benefit of training was its ability to counteract damages that were described in aging, through enhanced DNA repair and telomere preservation in the spinal cord (see fig. 18, 22 and 24; Safdar et al., 2011; Borghini et al., 2015).

Interestingly, exercise regulated the expression of numerous genes coding for RNA binding proteins (data not shown), paralleled by an increased activity in stress granules (fig. 16 and 24). This may indicate the activation of a protective mechanism able to prevent the formation of protein aggregates toxic for the cell (Kiebler and Bassell, 2006; Thomas et al., 2011). Furthermore, training modulated the expression of various genes implicated in ALS, Parkinson or Alzheimer disease (data not shown) and apoptotic signals were regulated in favor of intrinsic signals over extrinsic signals (fig. 18 and 22). Unexpectedly, the gene expression of the pro-survival kinase AKT was down-regulated after voluntary wheel-running in muscle as well as in the spinal cord (see table 3). Other studies have shown that AKT inhibits autophagy, and inhibits apoptosis induced by increased mitophagy in hepatocellular carcinoma (Wang et al., 2012; Liu et al., 2014). Thus decreased AKT pathway may be a protective mechanism that promotes intrinsic pro-apoptotic signals necessary for autophagy, the selective elimination of damaged cellular parts. Consistently with 
anterior studies, these results suggest the promotion of neuronal survival upon training (Cotman and Berchtold, 2002).

Importantly, the data indicate that nucleic acid regulation was enhanced upon endurance training. Notably, chromatin remodeling through histone modifications may be linked to the increased regulation of gene transcription (see fig. 14, 15, 18, 19, 22, 23 and 24; Barrès et al., 2012). Several studies reported epigenetic modulations upon physical exercise, which, in turn, control the expression of genes involved in metabolic adaptations (reviewed in Pareja-Galeano et al., 2014). For example, the down-regulation of histone deacetylase HDAC5-encoding gene coupled with the up-regulation of the transcription factor MEF2-encoding gene suggests the regulation of diverse metabolic genes, including $P g c-1 \alpha$, a master regulator of mitochondrial biogenesis and fatty acid oxidation (see table 3; Czubryt et al., 2003). Moreover, the loss of expression of histone demethylase KDM5A-encoding gene increases mitochondrial respiration through the repression of metabolic regulatory genes (Váraljai et al., 2015). Thus, the exercise-induced up-regulation of Kdm5a correlates with the apparent general reduction in mitochondrial oxidoreductase activity (see table 3 and fig. 26). Conversely, metabolic changes during exercise may also affect gene expression through epigenetic mechanisms. For instance, the balance of tricarboxylic acid cycle (TCA cycle or Krebs cycle) reactions determines the abundance of specific metabolites, which differently regulate the activity of the major DNA and histone demethylases TET1 and TET2 (Salminen et al., 2014). The down-regulation of Tet1 and Tet2 expression combined with the reduced activity of the TCA cycle (see fig. 14 and 22) may thus affect the level of DNA and histone methylation and thereby control gene expression linked to exercise regulation. Further, the expression of transcription factors was also directly modulated upon endurance training. The nuclear receptor ERR1 appears to be dispensable for basal cellular function; however, its target genes are required for optimal adaptation to exercise (Perry et al., 2014). Unexpectedly, the expression of Esrra, the ERR1encoding gene, was down-regulated following exercise (see table 3). One could speculate that the duration of the training was long enough for the system to adapt so that the running condition has become the basal condition and target gene transcription by ERR1 is no longer required. Interestingly, PROX1 that is specifically implicated in slow fiber-type specification in muscle is a negative modulator of ERR1, 
which is consistent with the increased Prox 1 but decreased Esrra gene expression in the present study (table 3 and fig. 27B; Charest-Marcotte et al., 2010). In line with enhanced gene transcription regulation, RNA splicing activity appeared increased following exercise (see fig. 14-26).

Besides, during exercise, both oxidative stress and endoplasmic reticulum (ER) stress are increased leading to abnormal mitochondrion and ER functioning. Several studies showed that mitophagy, the selective autophagy of damaged mitochondrial parts, was promoted by endurance training in the brain, skeletal muscle and other peripheral tissues (He et al., 2012b; Grumati et al., 2014; Sanchez et al., 2014). This suggests that mitophagy could be a mechanism by which exercise mediates mitochondrial turnover and improves resistance to oxidative stress, and one could speculate that exercise-induced ribophagy (the specific degradation of ribosomes) and reticulophagy (the selective elimination of portions of the ER) may be involved in enhanced translation efficiency (Jain et al., 2013). However, surprisingly, following voluntary training, gene expression of autophagic markers and oxidative stress markers was either not differentially regulated (most of the Atg gene family, or Fos, Fosb, Jun and Junb) or down-regulated (Mfn2, Mul1, Rab7 and Atg7, or metallothioneins Mt1 and Mt3). Moreover, two major consequences of endurance training were the apparent decrease in ER activity, comprising translation and ribosomal complex assembly, and repression of the mitochondrion activity that notably includes mitochondrial respiration (see fig. 14-25 and fig. 26). It was demonstrated that exercise promotes oxidation of BCAAs that are believed to serve as energy sources, substrates in the TCA cycle and to promote protein synthesis (Shimomura et al., 2004). The catabolism of BCAAs depends on the mitochondrial enzymatic complex BCKDH, which activity is activated by endurance exercise and which expression is increased by PGC-1 $\alpha$ (Hatzawa et al., 2014). However, the gene expression of BCKDH E1-a polypeptide was down-regulated following 12 weeks of voluntary wheel running (see table 3). Gene expression of ETHE1, a mitochondrial dioxygenase that is part of the OXPHOS complex, is positively correlated with the percentage of type I fibers in muscle (Parikh et al., 2008). Unexpectedly, Ethe1 expression was decreased in the present study, although the training induced a shift towards increased type I fibers in the soleus (see table 3, fig. 2 and 27). Moreover, the expression of Mlycd was down-regulated by voluntary exercise, while another 
study showed its increase in expression and protein activity upon exercise (see table 3; Kuhl et al., 2006). Finally, studies indicate that RRM2B activity decreases inflammation signals, suppresses the activation of the oxidative stress pathway and regulates mitochondrial activity (Chang et al., 2013; Kuo et al., 2012; Cho et al. 2015). Thus, the increased gene expression of the ribonucleotide reductase RRM2B is consistent with the observation of reduced mitochondrial respiration activity and decreased response to inflammation signals (see table3 and fig.14-25).

Taken together these results suggest that in contrast to what was previously reported in exercise studies, exercise may decrease oxidative stress, by upregulating the expression of oxidative stress pathway inhibitors, by down-regulating the expression of genes involved in mitochondrial oxidoreductase activity, or by regulating the expression of genes implicated in the modulation of mitochondrial gene programs. In addition, ER stress may also be reduced by a decrease in protein synthesis. Further, these results suggest that the changes of the transcriptome observed after prolonged endurance training, which are long-term adaptations, may therefore contrast with acute responses to shorter training sessions or other types of exercise. Moreover, many of the pathways that appear to change upon exercise are interconnected, with certain genes being shared by more than one pathway or cellular process. For example, in addition to its function in mitochondrial metabolism, MEF2 is implicated in the transcription regulation of synaptic genes and also plays a key role in post-synaptic maturation that condition accurate pre-synaptic active zone localization (Flavell et al., 2008; Prokop et al., 1996).

\subsection{Changes at the NMJ - synapse assembly and mitochondrial network}

Studies have shown that endurance training improves neuromuscular transmission efficacy in slow as well as in fast muscles due to increased transmitter release (Desaulniers et al., 2001; Argaw et al., 2004; Dorlöchter et al., 1991). It is also known that synaptic activity is a determinant of mitochondrial shape and organization, and reciprocally, changes in mitochondrial shape impact calcium signaling, which influences synaptic transmission (Brodin et al., 1999; Szabadkai et al, 2004; Billups and Forsythe, 2002). In order to address the characteristics of 
synaptic mitochondrial network remodeling upon exercise, I performed a histological analysis of NMJs in the predominantly slow fiber-containing soleus and the predominantly fast fiber-containing tibialis anterior (TA). Soleus NMJs had a larger mitochondrial volume and a higher mitochondrial concentration than TA NMJs, consistently with the report from crustacean NMJs showing that tonic synapses have more mitochondria than phasic synapses (see fig. 8; Nguyen et al., 1997). In addition, mitochondria clusters were elongated and connected in soleus NMJs, whereas the mitochondrial network of TA NMJs appeared fragmented, with small and spherical clusters (see fig. 9 and 10). These characteristics were modified by 12 weeks of voluntary running.

Firstly, the data show that exercise induced a different remodeling of the NMJ morphology in slow and fast muscles. While training elicited hypertrophy of slow post-synapses, fast NMJs were reduced in size (see fig. 7). Studies reported an increase in NMJ size in slow muscles correlated with the hypertrophy of slow muscle fibers after exercise (Waerhaug et al., 1992; Deschenes et al., 1993). However, the changes observed were only due to modifications of the length but not the width of the branches, indicating a specific adaptation rather than the simple stretch of the NMJ structure (see fig. 7). The repression of Rab7 gene expression observed following training may participate to this phenotype (see fig. 28). Indeed, another study demonstrated that a loss of presynaptic RAB7 leads to an increase in NMJ length (Lee et al., 2013). In addition, NMJ remodeling also occurs in sedentary healthy animals and likely correlates with muscle use, with slow NMJs undergoing more changes than fast NMJs (Wigston, 1989; Wigston 1990). Thus, the preferential recruitment of slow over fast motor units for endurance running may underpin the different adaptations observed between soleus and TA. In addition, since the size of the post-synapse was measured by labeling the acetylcholine receptors (AChRs) on the muscle membrane, a change in its size may be reflected by a change in number of AChRs. In contrast with the report that AChR number is increased in muscle fibers following exercise, the present study shows no modification in the expression of AChR gene family (data not shown; Desaulniers at al., 1998). However, gene expression level of Dystrophin, which is required for organizing large AChR aggregates on the post-synapse, was increased in soleus and in the ventral spinal cord (see fig. 28; Kong and Anderson, 1999). This suggests that endurance training 
affects the clustering rather than the number of AChRs and that exercise may promote pathways important for the strengthening of the post-synapse.

Secondly, enhanced activity maintained mitochondrial density in the presynapse by adjusting mitochondrial volume according to the change in NMJ size (see fig. 8). On one hand mitochondria subcellular distribution is dependent on cellular needs for ATP production and calcium signaling regulation, on the other hand neurotransmitter vesicle release requires high amounts of ATP and calcium signaling, and is increased during exercise (Verstreken et al, 2005). This suggests that pre-synaptic mitochondrial distribution may be altered by chronic activity, and that mitochondria may accumulate at the synapse upon enhanced synaptic activity. This hypothesis is consistent with the increased mitochondrial volume observed in trained soleus NMJs (see fig. 8). The maintenance of pre-synaptic mitochondria density and volume according to the change in NMJ size also suggests that mitochondrial biogenesis occurs in the slow NMJs and that elimination or redistribution of mitochondria takes place in the fast NMJs. Neurons utilize specialized mechanisms to regulate mitochondrial transport and retention near synaptic terminals, far away from the cell body (Cai et al., 2011). Interestingly, endurance training increased gene expression of Ranbp2, which is specifically implicated in the kinesin-dependent mitochondrion transport, but decreased gene expression of the kinesin KIFC3 that is present at synapses and mediates anterograde transport for proper subcellular distribution of mitochondria (see fig. 28; Cho et al., 2007; Hoang et al., 1999; Dietrich et al., 2013). Moreover, another study has shown that mitochondrial biogenesis is not limited to the cell body but that mitochondria can replicate their DNA, divide, and fuse locally within axons (Amiri and Hollenbeck, 2008). This suggests that chronic activity may regulate mitochondrial mobility and distribution at the motor endplate to adapt its metabolism to increased synaptic activity.

Thirdly, upon exercise, TA NMJs had a decreased normalized number of clusters, while the density of mitochondria remained unchanged (see fig. 9). This result indicates that the reduction of mitochondria cluster number is not only due to the reduction of TA NMJ size with exercise. Mitochondrial network was reshaped in fast NMJs in the direction of a more connected system, which may be the result of an increased fusion rate. The transcriptomic results provide evidence for the regulation 
of the fission-fusion equilibrium as shown by the increased expression of Yme1/1 (fig. 28; Anand et al., 2014). However, the data did not indicate whether the balance was tipped in favor of one or the other, since gene expression of the mitochondrial fusion factor Mitofusin-2 and of the promoter of mitochondrial fragmentation MUL1 were both down-regulated (table 3; Bach et al., 2003; Lokireddy et al., 2012). It was previously demonstrated that the fission/fusion ratio alone is insufficient to explain mitochondrial morphology because the rate of fission exceeds the rate of fusion in healthy neurons, which is compensated by the growth of mitochondrial organelles (Berman et al. 2009). However, there was no increase in average mitochondria cluster volume in TA NMJs (see fig. 10). This may be due to the fact that there are still many clusters in exercised TA NMJs. The additional volume per cluster may not be large enough to be considered significantly higher compared to the control condition.

Finally, the data indicate that the connectivity of the mitochondrial network is correlated to the density of mitochondria. When the mitochondrial volume increases in the endplate, the space is reduced and the chance that clusters connect each other may increase. In addition, mitochondria morphology and distribution influences synaptic morphology and plasticity, and reciprocally, synaptic activity is a determinant of mitochondria shape and organization (Berman et al., 2009; Li et al, 2004). The computational functional analysis showed that synaptic transmission was positively impacted by exercise, which is further supported by the regulated expression of various gene implicated in synaptic assembly and function (see fig. 14-25 and fig. 28). This suggests that the observed changes may not just be adaptations of the presynapse to the change in post-synaptic size, but rather specific modifications elicited in MNs in response to increased synaptic activity. Thus neuronal mitochondria may play important role in regulating NMJ strength and function upon chronic physical activity. Taken together, these results suggest a specific role for mitochondria in adapting synaptic organization and transmission to the increase in $\mathrm{MN}$ firing and muscle contraction elicited by exercise training.

Collectively, the results indicate a common adaptive response of the different components of the neuromuscular system to physical exercise. Although enhanced chromatin remodeling and regulation of transcription in response to exercise are well 
established, the impact of exercise on oxidative stress remains controversial. Indeed, it is well established that muscle contractions during exercise lead to elevated levels of reactive oxygen species, whereas exercise is well known to delay aging impairments by the reduction of oxidative stress and inflammation notably (Steinbacher and Eckl, 2015; Vincent et al., 2012). The present study provides evidence that oxidative stress, and potentially ER stress, may be reduced upon exercise in healthy animals. Moreover, interconnection of the pathways suggests that this may be mediated by a synergistic adaptation of the different cellular compartment organization and function.

In addition, the results indicate adaptation of NMJ morphology and presynaptic mitochondrial network in response to elevated physical activity, which was paralleled by specific synaptic gene expression changes in the soleus muscle and the ventral spinal cord. These genes are implicated in synaptic structure maintenance and synaptic transmission on one hand, and in mitochondrial morphology and subcellular distribution on the other hand. The regulation of mitochondrial biogenesis and dynamics may thus be tightly linked to the adaptation of NMJ structure and function to exercise. 


\section{Conclusion and outlook}

Numerous neurological and psychiatric disorders are associated with mitochondrial dysfunction, and include motor neuron diseases (Vos et al., 2010). Exercise proved to be beneficial in various chronic diseases as well as in degenerative conditions, including motor neuron diseases (Deforges et al., 2009; Carreras et al., 2010). A better understanding of mitochondrial biology and its regulation by physical activity may lead to novel therapeutic approaches and more broadly demonstrate the significance of "whole-body" benefits of exercise for society at large. The present study proposes that oxidative stress, and possibly ER stress, is reduced upon chronic endurance training in healthy mice. Physical activity may increase oxidative stress and rapidly elicit defense mechanisms such as mitophagy in order to limit cellular damages. Since the control of cellular stress has a cost in terms of energy consumption, mechanisms for the optimization of cellular functions under minimum energy expenditure may be elicited on the long term. The energy saving system may be mediated by decreased mitochondrial respiration and oxygen consumption, thus decreasing oxidative stress.

Exercise perturbs whole-body homeostasis, and ultimately elicits various adjustments in every organ. The present study shows that different tissues -muscle and spinal cord - share common mechanisms to adapt to chronic activity. The extension of the analysis to other tissues and samples, e.g. blood, heart or liver, will allow the identification of novel mechanisms of tissue crosstalk in response to exercise training. Organ crosstalk may also be achieved by the release into circulation of MicroRNAs (miRNAs) in exosomes for delivery to other tissues (Valadi et al., 2007). miRNAs are a class of small non-coding RNAs that regulate gene expression at the post-transcriptional level. A study reported the implication of miRNAs in exercise-mediated skeletal muscle remodeling (Safdar et al., 2009). Thus, future investigation of miRNA expression after exercise will contribute to our understanding of organ-organ communication and synchronized adaptation in the entire body. It is also important to note that modifications in gene expression cannot 
account for all the changes that occur in response to chronic activity. Indeed, the function of the cell not only depends on the level of gene transcription but also on the level of mRNA translation as well as protein function, i.e. activation or repression. In addition, protein lifetime and turnover rate may be regulated to minimize energy consumption. Therefore, an experimental functional analysis would complement the study by translating the observed transcriptional modifications into cellular functions. Finally, a study of mitochondrial dynamics, i.e. change in size, shape, and position of mitochondria over time within cells, will complete our understanding of the exerciseinduced modulation of the mitochondrial network in NMJs. This will be achieved using in vivo 2-photon FRAP (Fluorescence Recovery After Photobleaching) experiments in MDO mouse muscles.

The combination of these studies will contribute to our comprehension of "whole-body" metabolic regulations and mitochondrial function upon neuromuscular activity, and may ultimately provide further insights into the mechanisms that underlie neuroprotective effects of exercise. 


\section{References}

Adler, M., Sweeney, R.E., Hamilton, T.A., Lockridge, O., Duysen, E.G., Purcell, A.L., Deshpande, S.S., 2011. Role of acetylcholinesterase on the structure and function of cholinergic synapses: insights gained from studies on knockout mice. Cell. Mol. Neurobiol. 31, 909-920.

Agudelo, L.Z., Femenía, T., Orhan, F., Porsmyr-Palmertz, M., Goiny, M., Martinez-Redondo, V., Correia, J.C., Izadi, M., Bhat, M., Schuppe-Koistinen, I., Pettersson, A.T., Ferreira, D.M.S., Krook, A., Barres, R., Zierath, J.R., Erhardt, S., Lindskog, M., Ruas, J.L., 2014. Skeletal muscle PGC-1a1 modulates kynurenine metabolism and mediates resilience to stress-induced depression. Cell 159, 33-45.

Akimoto, T., Pohnert, S.C., Li, P., Zhang, M., Gumbs, C., Rosenberg, P.B., Williams, R.S., Yan, Z., 2005. Exercise stimulates Pgc-1alpha transcription in skeletal muscle through activation of the p38 MAPK pathway. J. Biol. Chem. 280, 19587-19593.

Alaynick, W.A., Jessell, T.M., Pfaff, S.L., 2011. SnapShot: spinal cord development. Cell 146, 178178.e1.

Amiri, M., Hollenbeck, P.J., 2008. Mitochondrial biogenesis in the axons of vertebrate peripheral neurons. Dev Neurobiol 68, 1348-1361.

Anand, R., Wai, T., Baker, M.J., Kladt, N., Schauss, A.C., Rugarli, E., Langer, T., 2014. The i-AAA protease YME1L and OMA1 cleave OPA1 to balance mitochondrial fusion and fission. J. Cell Biol. 204, 919-929.

Anders, S., Pyl, P.T., Huber, W., 2015. HTSeq--a Python framework to work with high-throughput sequencing data. Bioinformatics 31, 166-169.

Andonian, M.H., Fahim, M.A., 1988. Endurance exercise alters the morphology of fast- and slowtwitch rat neuromuscular junctions. Int J Sports Med 9, 218-223.

Angus, L.M., Chakkalakal, J.V., Méjat, A., Eibl, J.K., Bélanger, G., Megeney, L.A., Chin, E.R., Schaeffer, L., Michel, R.N., Jasmin, B.J., 2005. Calcineurin-NFAT signaling, together with GABP and peroxisome PGC-1\{alpha\}, drives utrophin gene expression at the neuromuscular junction. Am. J. Physiol., Cell Physiol. 289, C908-917.

Anziska, Y., Sternberg, A., 2013. Exercise in neuromuscular disease. Muscle Nerve 48, 3-20.

Aoi, W., Naito, Y., Takagi, T., Tanimura, Y., Takanami, Y., Kawai, Y., Sakuma, K., Hang, L.P., Mizushima, K., Hirai, Y., Koyama, R., Wada, S., Higashi, A., Kokura, S., Ichikawa, H., Yoshikawa, T., 2013. A novel myokine, secreted protein acidic and rich in cysteine (SPARC), suppresses colon tumorigenesis via regular exercise. Gut 62, 882-889.

Argaw, A., Desaulniers, P., Gardiner, P.F., 2004. Enhanced neuromuscular transmission efficacy in overloaded rat plantaris muscle. Muscle Nerve 29, 97-103.

Arikkath, J., Campbell, K.P., 2003. Auxiliary subunits: essential components of the voltage-gated calcium channel complex. Curr. Opin. Neurobiol. 13, 298-307.

Ashburner, M., Ball, C.A., Blake, J.A., Botstein, D., Butler, H., Cherry, J.M., Davis, A.P., Dolinski, K., Dwight, S.S., Eppig, J.T., Harris, M.A., Hill, D.P., Issel-Tarver, L., Kasarskis, A., Lewis, S., Matese, J.C., Richardson, J.E., Ringwald, M., Rubin, G.M., Sherlock, G., 2000. Gene ontology: tool for the unification of biology. The Gene Ontology Consortium. Nat. Genet. 25, 25-29.

Ausoni, S., Gorza, L., Schiaffino, S., Gundersen, K., Lømo, T., 1990. Expression of myosin heavy chain isoforms in stimulated fast and slow rat muscles. J. Neurosci. 10, 153-160.

Baar, K., 2009. The signaling underlying FITness. Appl Physiol Nutr Metab 34, 411-419. 
Bach, D., Pich, S., Soriano, F.X., Vega, N., Baumgartner, B., Oriola, J., Daugaard, J.R., Lloberas, J., Camps, M., Zierath, J.R., Rabasa-Lhoret, R., Wallberg-Henriksson, H., Laville, M., Palacín, M., Vidal, H., Rivera, F., Brand, M., Zorzano, A., 2003. Mitofusin-2 determines mitochondrial network architecture and mitochondrial metabolism. A novel regulatory mechanism altered in obesity. J. Biol. Chem. 278, 17190-17197.

Bakels, R., Kernell, D., 1993. Matching between motoneurone and muscle unit properties in rat medial gastrocnemius. J. Physiol. (Lond.) 463, 307-324.

Balice-Gordon, R.J., Breedlove, S.M., Bernstein, S., Lichtman, J.W., 1990. Neuromuscular junctions shrink and expand as muscle fiber size is manipulated: in vivo observations in the androgen-sensitive bulbocavernosus muscle of mice. J. Neurosci. 10, 2660-2671.

Barrès, R., Yan, J., Egan, B., Treebak, J.T., Rasmussen, M., Fritz, T., Caidahl, K., Krook, A., O'Gorman, D.J., Zierath, J.R., 2012. Acute exercise remodels promoter methylation in human skeletal muscle. Cell Metab. 15, 405-411.

Bassel-Duby, R., Olson, E.N., 2006. Signaling pathways in skeletal muscle remodeling. Annu. Rev. Biochem. 75, 19-37.

Baylor, S.M., Hollingworth, S., 2003. Sarcoplasmic reticulum calcium release compared in slow-twitch and fast-twitch fibres of mouse muscle. J. Physiol. (Lond.) 551, 125-138.

Bayod, S., Del Valle, J., Canudas, A.M., Lalanza, J.F., Sanchez-Roige, S., Camins, A., Escorihuela, R.M., Pallàs, M., 2011. Long-term treadmill exercise induces neuroprotective molecular changes in rat brain. J. Appl. Physiol. 111, 1380-1390.

Beaumont, E., Gardiner, P., 2002. Effects of daily spontaneous running on the electrophysiological properties of hindlimb motoneurones in rats. J. Physiol. (Lond.) 540, 129-138.

Beaumont, E., Gardiner, P.F., 2003. Endurance training alters the biophysical properties of hindlimb motoneurons in rats. Muscle Nerve 27, 228-236.

Berman, S.B., Chen, Y., Qi, B., McCaffery, J.M., Rucker, E.B., Goebbels, S., Nave, K.-A., Arnold, B.A., Jonas, E.A., Pineda, F.J., Hardwick, J.M., 2009. Bcl-x L increases mitochondrial fission, fusion, and biomass in neurons. J. Cell Biol. 184, 707-719.

Bessou, P., Emonet-Denand, F., Laporte, Y., 1962. [Action of fusimotor fibers on the discharge of secondary endings of neuromuscular spindles, in the cat]. J. Physiol. (Paris) 54, 292-293.

Bhogal, B., Jepson, J.E., Savva, Y.A., Pepper, A.S.-R., Reenan, R.A., Jongens, T.A., 2011. Modulation of dADAR-dependent RNA editing by the Drosophila fragile $\mathrm{X}$ mental retardation protein. Nat. Neurosci. 14, 1517-1524.

Billups, B., Forsythe, I.D., 2002. Presynaptic mitochondrial calcium sequestration influences transmission at mammalian central synapses. J. Neurosci. 22, 5840-5847.

Bird, S.R., Hawley, J.A., 2012. Exercise and type 2 diabetes: new prescription for an old problem. Maturitas 72, 311-316.

Bloemberg, D., Quadrilatero, J., 2012. Rapid determination of myosin heavy chain expression in rat, mouse, and human skeletal muscle using multicolor immunofluorescence analysis. PLoS ONE 7, e35273.

Bodine, S.C., Stitt, T.N., Gonzalez, M., Kline, W.O., Stover, G.L., Bauerlein, R., Zlotchenko, E., Scrimgeour, A., Lawrence, J.C., Glass, D.J., Yancopoulos, G.D., 2001. Akt/mTOR pathway is a crucial regulator of skeletal muscle hypertrophy and can prevent muscle atrophy in vivo. Nat. Cell Biol. 3, 1014-1019.

Borghini, A., Giardini, G., Tonacci, A., Mastorci, F., Mercuri, A., Mrakic-Sposta, S., Sposta, S.M., Moretti, S., Andreassi, M.G., Pratali, L., 2015. Chronic and acute effects of endurance training on telomere length. Mutagenesis $30,711-716$. 
Boström, P., Wu, J., Jedrychowski, M.P., Korde, A., Ye, L., Lo, J.C., Rasbach, K.A., Boström, E.A., Choi, J.H., Long, J.Z., Kajimura, S., Zingaretti, M.C., Vind, B.F., Tu, H., Cinti, S., Højlund, K., Gygi, S.P., Spiegelman, B.M., 2012. A PGC1-a-dependent myokine that drives brown-fat-like development of white fat and thermogenesis. Nature 481, 463-468.

Bottinelli, R., Betto, R., Schiaffino, S., Reggiani, C., 1994a. Maximum shortening velocity and coexistence of myosin heavy chain isoforms in single skinned fast fibres of rat skeletal muscle. J. Muscle Res. Cell. Motil. 15, 413-419.

Bottinelli, R., Canepari, M., Reggiani, C., Stienen, G.J., 1994b. Myofibrillar ATPase activity during isometric contraction and isomyosin composition in rat single skinned muscle fibres. J. Physiol. (Lond.) 481 (Pt 3), 663-675.

Bretag, A.H., 1987. Muscle chloride channels. Physiol. Rev. 67, 618-724.

Brodin, L., Bakeeva, L., Shupliakov, O., 1999. Presynaptic mitochondria and the temporal pattern of neurotransmitter release. Philos. Trans. R. Soc. Lond., B, Biol. Sci. 354, 365-372.

Brown, J.C., Winters-Stone, K., Lee, A., Schmitz, K.H., 2012. Cancer, physical activity, and exercise. Compr Physiol 2, 2775-2809.

Bruusgaard, J.C., Johansen, I.B., Egner, I.M., Rana, Z.A., Gundersen, K., 2010. Myonuclei acquired by overload exercise precede hypertrophy and are not lost on detraining. Proc. Natl. Acad. Sci. U.S.A. $107,15111-15116$.

Buchthal, F., Schmalbruch, H., 1980. Motor unit of mammalian muscle. Physiol. Rev. 60, 90-142.

Buller, A.J., Eccles, J.C., Eccles, R.M., 1960. Interactions between motoneurones and muscles in respect of the characteristic speeds of their responses. J. Physiol. (Lond.) 150, 417-439.

Burke, R.E., Levine, D.N., Tsairis, P., Zajac, F.E., 1973. Physiological types and histochemical profiles in motor units of the cat gastrocnemius. J. Physiol. (Lond.) 234, 723-748.

Cai, Q., Davis, M.L., Sheng, Z.-H., 2011. Regulation of axonal mitochondrial transport and its impact on synaptic transmission. Neurosci. Res. 70, 9-15.

Carreras, I., Yuruker, S., Aytan, N., Hossain, L., Choi, J.-K., Jenkins, B.G., Kowall, N.W., Dedeoglu, A., 2010. Moderate exercise delays the motor performance decline in a transgenic model of ALS. Brain Res. 1313, 192-201.

Carroll, S.L., Klein, M.G., Schneider, M.F., 1997. Decay of calcium transients after electrical stimulation in rat fast- and slow-twitch skeletal muscle fibres. J. Physiol. (Lond.) 501 (Pt 3), 573-588.

Celichowski, J., Krutki, P., Bichler, E., 1996. Axonal conduction velocity of motor units of rat's medial gastrocnemius muscle. J. Physiol. Paris 90, 75-78.

Chakkalakal, J.V., Nishimune, H., Ruas, J.L., Spiegelman, B.M., Sanes, J.R., 2010. Retrograde influence of muscle fibers on their innervation revealed by a novel marker for slow motoneurons. Development 137, 3489-3499.

Chang, L., Guo, R., Huang, Q., Yen, Y., 2013. Chromosomal instability triggered by Rrm2b loss leads to IL-6 secretion and plasmacytic neoplasms. Cell Rep 3, 1389-1397.

Charest-Marcotte, A., Dufour, C.R., Wilson, B.J., Tremblay, A.M., Eichner, L.J., Arlow, D.H., Mootha, V.K., Giguère, V., 2010. The homeobox protein Prox1 is a negative modulator of ERR\{alpha\}/PGC1 \{alpha\} bioenergetic functions. Genes Dev. 24, 537-542.

Cho, E.-C., Kuo, M.-L., Cheng, J.-H., Cheng, Y.-C., Hsieh, Y.-C., Liu, Y.-R., Hsieh, R.-H., Yen, Y., 2015. RRM2B-Mediated Regulation of Mitochondrial Activity and Inflammation under Oxidative Stress. Mediators Inflamm. 2015, 287345.

Cho, K., Cai, Y., Yi, H., Yeh, A., Aslanukov, A., Ferreira, P.A., 2007. Association of the kinesin-binding domain of RanBP2 to KIF5B and KIF5C determines mitochondria localization and function. Traffic 8, $1722-1735$. 
Close, R., 1967. Properties of motor units in fast and slow skeletal muscles of the rat. J. Physiol. (Lond.) 193, 45-55.

Cohen, T.V., Randall, W.R., 2004. NFATc1 activates the acetylcholinesterase promoter in rat muscle. J. Neurochem. 90, 1059-1067.

Cole, R.P., 1982. Myoglobin function in exercising skeletal muscle. Science 216, 523-525.

Cotman, C.W., Berchtold, N.C., 2002. Exercise: a behavioral intervention to enhance brain health and plasticity. Trends Neurosci. 25, 295-301.

Crabtree, G.R., Schreiber, S.L., 2009. SnapShot: Ca2+-calcineurin-NFAT signaling. Cell 138, 210, 210.e1.

Cramer, P., Cáceres, J.F., Cazalla, D., Kadener, S., Muro, A.F., Baralle, F.E., Kornblihtt, A.R., 1999. Coupling of transcription with alternative splicing: RNA pol II promoters modulate SF2/ASF and 9G8 effects on an exonic splicing enhancer. Mol. Cell 4, 251-258.

Cullheim, S., Fleshman, J.W., Glenn, L.L., Burke, R.E., 1987. Membrane area and dendritic structure in type-identified triceps surae alpha motoneurons. J. Comp. Neurol. 255, 68-81.

Czubryt, M.P., McAnally, J., Fishman, G.I., Olson, E.N., 2003. Regulation of peroxisome proliferatoractivated receptor gamma coactivator 1 alpha (PGC-1 alpha) and mitochondrial function by MEF2 and HDAC5. Proc. Natl. Acad. Sci. U.S.A. 100, 1711-1716.

De Luca, C.J., Contessa, P., 2012. Hierarchical control of motor units in voluntary contractions. J. Neurophysiol. 107, 178-195.

Deblois, G., Giguère, V., 2011. Functional and physiological genomics of estrogen-related receptors (ERRs) in health and disease. Biochim. Biophys. Acta 1812, 1032-1040.

Deforges, S., Branchu, J., Biondi, O., Grondard, C., Pariset, C., Lécolle, S., Lopes, P., Vidal, P.-P., Chanoine, C., Charbonnier, F., 2009. Motoneuron survival is promoted by specific exercise in a mouse model of amyotrophic lateral sclerosis. J. Physiol. (Lond.) 587, 3561-3572.

Delp, M.D., Duan, C., 1996. Composition and size of type I, IIA, IID/X, and IIB fibers and citrate synthase activity of rat muscle. J. Appl. Physiol. 80, 261-270.

Desaulniers, P., Lavoie, P.A., Gardiner, P.F., 2001. Habitual exercise enhances neuromuscular transmission efficacy of rat soleus muscle in situ. J. Appl. Physiol. 90, 1041-1048.

Desaulniers, P., Lavoie, P.A., Gardiner, P.F., 1998. Endurance training increases acetylcholine receptor quantity at neuromuscular junctions of adult rat skeletal muscle. Neuroreport 9, 3549-3552.

Deschenes, M.R., Maresh, C.M., Crivello, J.F., Armstrong, L.E., Kraemer, W.J., Covault, J., 1993. The effects of exercise training of different intensities on neuromuscular junction morphology. J. Neurocytol. 22, 603-615.

Dessaud, E., Yang, L.L., Hill, K., Cox, B., Ulloa, F., Ribeiro, A., Mynett, A., Novitch, B.G., Briscoe, J., 2007. Interpretation of the sonic hedgehog morphogen gradient by a temporal adaptation mechanism. Nature 450, 717-720.

Dietrich, D., Seiler, F., Essmann, F., Dodt, G., 2013. Identification of the kinesin KifC3 as a new player for positioning of peroxisomes and other organelles in mammalian cells. Biochim. Biophys. Acta 1833, 3013-3024.

Dobin, A., Davis, C.A., Schlesinger, F., Drenkow, J., Zaleski, C., Jha, S., Batut, P., Chaisson, M., Gingeras, T.R., 2013. STAR: ultrafast universal RNA-seq aligner. Bioinformatics 29, 15-21.

Dobrowolny, G., Giacinti, C., Pelosi, L., Nicoletti, C., Winn, N., Barberi, L., Molinaro, M., Rosenthal, N., Musarò, A., 2005. Muscle expression of a local lgf-1 isoform protects motor neurons in an ALS mouse model. J. Cell Biol. 168, 193-199. 
Dorlöchter, M., Irintchev, A., Brinkers, M., Wernig, A., 1991. Effects of enhanced activity on synaptic transmission in mouse extensor digitorum longus muscle. J. Physiol. (Lond.) 436, 283-292.

Dupont-Versteegden, E.E., Houlé, J.D., Dennis, R.A., Zhang, J., Knox, M., Wagoner, G., Peterson, C.A., 2004. Exercise-induced gene expression in soleus muscle is dependent on time after spinal cord injury in rats. Muscle Nerve 29, 73-81.

Edström, L., Grimby, L., 1986. Effect of exercise on the motor unit. Muscle Nerve 9, 104-126.

Edström, L., Kugelberg, E., 1968. Histochemical composition, distribution of fibres and fatiguability of single motor units. Anterior tibial muscle of the rat. J. Neurol. Neurosurg. Psychiatr. 31, 424-433.

Egan, B., Dowling, P., O'Connor, P.L., Henry, M., Meleady, P., Zierath, J.R., O'Gorman, D.J., 2011. 2D DIGE analysis of the mitochondrial proteome from human skeletal muscle reveals time coursedependent remodelling in response to 14 consecutive days of endurance exercise training. Proteomics $11,1413-1428$.

Egan, B., Zierath, J.R., 2013. Exercise metabolism and the molecular regulation of skeletal muscle adaptation. Cell Metab. 17, 162-184.

Eikelboom, R., Mills, R., 1988. A microanalysis of wheel running in male and female rats. Physiol. Behav. 43, 625-630.

Enjin, A., Rabe, N., Nakanishi, S.T., Vallstedt, A., Gezelius, H., Memic, F., Lind, M., Hjalt, T., Tourtellotte, W.G., Bruder, C., Eichele, G., Whelan, P.J., Kullander, K., 2010. Identification of novel spinal cholinergic genetic subtypes disclose Chodl and Pitx2 as markers for fast motor neurons and partition cells. J. Comp. Neurol. 518, 2284-2304.

Filézac de L'Etang, A., Maharjan, N., Cordeiro Braña, M., Ruegsegger, C., Rehmann, R., Goswami, A., Roos, A., Troost, D., Schneider, B.L., Weis, J., Saxena, S., 2015. Marinesco-Sjögren syndrome protein SIL1 regulates motor neuron subtype-selective ER stress in ALS. Nat. Neurosci. 18, 227-238.

Finn, A.J., Feng, G., Pendergast, A.M., 2003. Postsynaptic requirement for Abl kinases in assembly of the neuromuscular junction. Nat. Neurosci. 6, 717-723.

Flavell, S.W., Kim, T.-K., Gray, J.M., Harmin, D.A., Hemberg, M., Hong, E.J., MarkenscoffPapadimitriou, E., Bear, D.M., Greenberg, M.E., 2008. Genome-wide analysis of MEF2 transcriptional program reveals synaptic target genes and neuronal activity-dependent polyadenylation site selection. Neuron 60, 1022-1038.

Forsgren, S., Bergh, A., Carlsson, E., Thornell, L.E., 1993. Calcitonin gene-related peptide expression at endplates of different fibre types in muscles in rat hind limbs. Cell Tissue Res. 274, 439-446.

Frey, D., Schneider, C., Xu, L., Borg, J., Spooren, W., Caroni, P., 2000. Early and selective loss of neuromuscular synapse subtypes with low sprouting competence in motoneuron diseases. J. Neurosci. 20, 2534-2542.

Funakoshi, H., Belluardo, N., Arenas, E., Yamamoto, Y., Casabona, A., Persson, H., Ibáñez, C.F., 1995. Muscle-derived neurotrophin-4 as an activity-dependent trophic signal for adult motor neurons. Science 268, 1495-1499.

Gardiner, P., Dai, Y., Heckman, C.J., 2006. Effects of exercise training on alpha-motoneurons. J. Appl. Physiol. 101, 1228-1236.

Gardiner, P.F., 1993. Physiological properties of motoneurons innervating different muscle unit types in rat gastrocnemius. J. Neurophysiol. 69, 1160-1170.

Gharakhanlou, R., Chadan, S., Gardiner, P., 1999. Increased activity in the form of endurance training increases calcitonin gene-related peptide content in lumbar motoneuron cell bodies and in sciatic nerve in the rat. Neuroscience 89, 1229-1239.

Gómez-Pinilla, F., Ying, Z., Roy, R.R., Molteni, R., Edgerton, V.R., 2002. Voluntary exercise induces a BDNF-mediated mechanism that promotes neuroplasticity. J. Neurophysiol. 88, 2187-2195. 
Gonzalez, M., Collins, W.F., 1997. Modulation of motoneuron excitability by brain-derived neurotrophic factor. J. Neurophysiol. 77, 502-506.

Gonzalez-Freire, M., de Cabo, R., Studenski, S.A., Ferrucci, L., 2014. The Neuromuscular Junction: Aging at the Crossroad between Nerves and Muscle. Front Aging Neurosci 6, 208.

Goodyear, L.J., Giorgino, F., Balon, T.W., Condorelli, G., Smith, R.J., 1995. Effects of contractile activity on tyrosine phosphoproteins and PI 3-kinase activity in rat skeletal muscle. Am. J. Physiol. 268, E987-995.

Gordon, T., Tyreman, N., Rafuse, V.F., Munson, J.B., 1997. Fast-to-slow conversion following chronic low-frequency activation of medial gastrocnemius muscle in cats. I. Muscle and motor unit properties. J. Neurophysiol. 77, 2585-2604.

Goulding, M., 2009. Circuits controlling vertebrate locomotion: moving in a new direction. Nat. Rev. Neurosci. 10, 507-518.

Grondard, C., Biondi, O., Armand, A.-S., Lécolle, S., Della Gaspera, B., Pariset, C., Li, H., Gallien, C.L., Vidal, P.-P., Chanoine, C., Charbonnier, F., 2005. Regular exercise prolongs survival in a type 2 spinal muscular atrophy model mouse. J. Neurosci. 25, 7615-7622.

Grumati, P., Coletto, L., Schiavinato, A., Castagnaro, S., Bertaggia, E., Sandri, M., Bonaldo, P., 2011. Physical exercise stimulates autophagy in normal skeletal muscles but is detrimental for collagen VIdeficient muscles. Autophagy 7, 1415-1423.

Hameed, M., Orrell, R.W., Cobbold, M., Goldspink, G., Harridge, S.D.R., 2003. Expression of IGF-I splice variants in young and old human skeletal muscle after high resistance exercise. J. Physiol. (Lond.) 547, 247-254.

Handschin, C., Spiegelman, B.M., 2008. The role of exercise and PGC1alpha in inflammation and chronic disease. Nature 454, 463-469.

Harris, J.J., Jolivet, R., Attwell, D., 2012. Synaptic energy use and supply. Neuron 75, 762-777.

Hashimoto, K., Hayashi, Y., Inuzuka, T., Hozumi, I., 2009. Exercise induces metallothioneins in mouse spinal cord. Neuroscience 163, 244-251.

Hatazawa, Y., Tadaishi, M., Nagaike, Y., Morita, A., Ogawa, Y., Ezaki, O., Takai-Igarashi, T., Kitaura, Y., Shimomura, Y., Kamei, Y., Miura, S., 2014. PGC-1a-mediated branched-chain amino acid metabolism in the skeletal muscle. PLoS ONE 9, e91006.

Hatze, H., Buys, J.D., 1977. Energy-optimal controls in the mammalian neuromuscular system. Biol Cybern 27, 9-20.

Hawley, J.A., Hargreaves, M., Joyner, M.J., Zierath, J.R., 2014. Integrative biology of exercise. Cell $159,738-749$.

He, C., Sumpter, R., Levine, B., 2012. Exercise induces autophagy in peripheral tissues and in the brain. Autophagy 8, 1548-1551.

Heckman, C.J., Mottram, C., Quinlan, K., Theiss, R., Schuster, J., 2009. Motoneuron excitability: the importance of neuromodulatory inputs. Clin Neurophysiol 120, 2040-2054.

Hegedus, J., Putman, C.T., Gordon, T., 2007. Time course of preferential motor unit loss in the SOD1 G93A mouse model of amyotrophic lateral sclerosis. Neurobiol. Dis. 28, 154-164.

Hegedus, J., Putman, C.T., Tyreman, N., Gordon, T., 2008. Preferential motor unit loss in the SOD1 G93A transgenic mouse model of amyotrophic lateral sclerosis. J. Physiol. (Lond.) 586, 3337-3351.

Henderson, C.E., Phillips, H.S., Pollock, R.A., Davies, A.M., Lemeulle, C., Armanini, M., Simmons, L., Moffet, B., Vandlen, R.A., Simpson LC corrected to Simmons, L., Koliatsos, V.E., Rosenthal, A., 1994. GDNF: a potent survival factor for motoneurons present in peripheral nerve and muscle. Science 266, 1062-1064. 
Hildebrandt, A.L., Pilegaard, H., Neufer, P.D., 2003. Differential transcriptional activation of select metabolic genes in response to variations in exercise intensity and duration. Am. J. Physiol. Endocrinol. Metab. 285, E1021-1027.

Hildebrandt, T.M., Di Meo, I., Zeviani, M., Viscomi, C., Braun, H.-P., 2013. Proteome adaptations in Ethe1-deficient mice indicate a role in lipid catabolism and cytoskeleton organization via posttranslational protein modifications. Biosci. Rep. 33.

Hoang, E., Bost-Usinger, L., Burnside, B., 1999. Characterization of a novel C-kinesin (KIFC3) abundantly expressed in vertebrate retina and RPE. Exp. Eye Res. 69, 57-68.

Hollmann, W., Strüder, H.K., Tagarakis, C.V.M., King, G., 2007. Physical activity and the elderly. Eur J Cardiovasc Prev Rehabil 14, 730-739.

Izumiya, Y., Hopkins, T., Morris, C., Sato, K., Zeng, L., Viereck, J., Hamilton, J.A., Ouchi, N., LeBrasseur, N.K., Walsh, K., 2008. Fast/Glycolytic muscle fiber growth reduces fat mass and improves metabolic parameters in obese mice. Cell Metab. 7, 159-172.

Jain, M.V., Paczulla, A.M., Klonisch, T., Dimgba, F.N., Rao, S.B., Roberg, K., Schweizer, F., Lengerke, C., Davoodpour, P., Palicharla, V.R., Maddika, S., Łos, M., 2013. Interconnections between apoptotic, autophagic and necrotic pathways: implications for cancer therapy development. J. Cell. Mol. Med. 17, 12-29.

Jasmin, B.J., Lavoie, P.A., Gardiner, P.F., 1988. Fast axonal transport of labeled proteins in motoneurons of exercise-trained rats. Am. J. Physiol. 255, C731-736.

Jasmin, B.J., Lavoie, P.A., Gardiner, P.F., 1987. Fast axonal transport of acetylcholinesterase in rat sciatic motoneurons is enhanced following prolonged daily running, but not following swimming. Neurosci. Lett. 78, 156-160.

Kang, C.M., Lavoie, P.A., Gardiner, P.F., 1995. Chronic exercise increases SNAP-25 abundance in fast-transported proteins of rat motoneurones. Neuroreport 6, 549-553.

Kanning, K.C., Kaplan, A., Henderson, C.E., 2010. Motor neuron diversity in development and disease. Annu. Rev. Neurosci. 33, 409-440.

Kaplan, A., Spiller, K.J., Towne, C., Kanning, K.C., Choe, G.T., Geber, A., Akay, T., Aebischer, P., Henderson, C.E., 2014. Neuronal matrix metalloproteinase-9 is a determinant of selective neurodegeneration. Neuron 81, 333-348.

Keller-Peck, C.R., Feng, G., Sanes, J.R., Yan, Q., Lichtman, J.W., Snider, W.D., 2001. Glial cell linederived neurotrophic factor administration in postnatal life results in motor unit enlargement and continuous synaptic remodeling at the neuromuscular junction. J. Neurosci. 21, 6136-6146.

Kernell, D., 2003. Principles of force gradation in skeletal muscles. Neural Plast. 10, 69-76.

Kernell, D., Bakels, R., Copray, J.C., 1999. Discharge properties of motoneurones: how are they matched to the properties and use of their muscle units? J. Physiol. Paris 93, 87-96.

Kiebler, M.A., Bassell, G.J., 2006. Neuronal RNA granules: movers and makers. Neuron 51, 685-690.

Kirn-Safran, C.B., Oristian, D.S., Focht, R.J., Parker, S.G., Vivian, J.L., Carson, D.D., 2007. Global growth deficiencies in mice lacking the ribosomal protein HIP/RPL29. Dev. Dyn. 236, 447-460.

Koga, K., Liu, M.-G., Qiu, S., Song, Q., O’Den, G., Chen, T., Zhuo, M., 2015. Impaired presynaptic long-term potentiation in the anterior cingulate cortex of Fmr1 knock-out mice. J. Neurosci. 35, 20332043.

Koliatsos, V.E., Clatterbuck, R.E., Winslow, J.W., Cayouette, M.H., Price, D.L., 1993. Evidence that brain-derived neurotrophic factor is a trophic factor for motor neurons in vivo. Neuron 10, 359-367.

Kong, J., Anderson, J.E., 1999. Dystrophin is required for organizing large acetylcholine receptor aggregates. Brain Res. 839, 298-304. 
Krutki, P., Hałuszka, A., Mrówczyński, W., Gardiner, P.F., Celichowski, J., 2015. Adaptations of motoneuron properties to chronic compensatory muscle overload. J. Neurophysiol. 113, 2769-2777.

Kuffler, S.W., Hunt, C.C., Quilliam, J.P., 1951. Function of medullated small-nerve fibers in mammalian ventral roots; efferent muscle spindle innervation. J. Neurophysiol. 14, 29-54.

Kuhl, J.E., Ruderman, N.B., Musi, N., Goodyear, L.J., Patti, M.E., Crunkhorn, S., Dronamraju, D., Thorell, A., Nygren, J., Ljungkvist, O., Degerblad, M., Stahle, A., Brismar, T.B., Andersen, K.L., Saha, A.K., Efendic, S., Bavenholm, P.N., 2006. Exercise training decreases the concentration of malonylCoA and increases the expression and activity of malonyl-CoA decarboxylase in human muscle. Am. J. Physiol. Endocrinol. Metab. 290, E1296-1303.

Kuo, M.-L., Sy, A.J., Xue, L., Chi, M., Lee, M.T.-C., Yen, T., Chiang, M.-I., Chang, L., Chu, P., Yen, Y., 2012. RRM2B suppresses activation of the oxidative stress pathway and is up-regulated by p53 during senescence. Sci Rep 2, 822.

Landmesser, L., 1978. The distribution of motoneurones supplying chick hind limb muscles. J. Physiol. (Lond.) 284, 371-389.

Lebrasseur, N.K., Coté, G.M., Miller, T.A., Fielding, R.A., Sawyer, D.B., 2003. Regulation of neuregulin/ErbB signaling by contractile activity in skeletal muscle. Am. J. Physiol., Cell Physiol. 284, C1149-1155.

Lee, H.-G., Zhao, N., Campion, B.K., Nguyen, M.M., Selleck, S.B., 2013. Akt regulates glutamate receptor trafficking and postsynaptic membrane elaboration at the Drosophila neuromuscular junction. Dev Neurobiol 73, 723-743.

Lee, M.-J., Jang, S., Nahm, M., Yoon, J.-H., Lee, S., 2013. Tbc1d15-17 regulates synaptic development at the Drosophila neuromuscular junction. Mol. Cells 36, 163-168.

Li, Q., Zheng, S., Han, A., Lin, C.-H., Stoilov, P., Fu, X.-D., Black, D.L., 2014. The splicing regulator PTBP2 controls a program of embryonic splicing required for neuronal maturation. Elife 3, e01201.

Li, Z., Okamoto, K.-I., Hayashi, Y., Sheng, M., 2004. The importance of dendritic mitochondria in the morphogenesis and plasticity of spines and synapses. Cell 119, 873-887.

Licatalosi, D.D., Yano, M., Fak, J.J., Mele, A., Grabinski, S.E., Zhang, C., Darnell, R.B., 2012. Ptbp2 represses adult-specific splicing to regulate the generation of neuronal precursors in the embryonic brain. Genes Dev. 26, 1626-1642.

Liu, K., Shi, Y., Guo, X.H., Ouyang, Y.B., Wang, S.S., Liu, D.J., Wang, A.N., Li, N., Chen, D.X., 2014. Phosphorylated AKT inhibits the apoptosis induced by DRAM-mediated mitophagy in hepatocellular carcinoma by preventing the translocation of DRAM to mitochondria. Cell Death Dis 5, e1078.

Liu-Yesucevitz, L., Bassell, G.J., Gitler, A.D., Hart, A.C., Klann, E., Richter, J.D., Warren, S.T., Wolozin, B., 2011. Local RNA translation at the synapse and in disease. J. Neurosci. 31, 1608616093.

Love, M.I., Huber, W., Anders, S., 2014. Moderated estimation of fold change and dispersion for RNAseq data with DESeq2. Genome Biol. 15, 550.

Lu, D.C., Niu, T., Alaynick, W.A., 2015. Molecular and cellular development of spinal cord locomotor circuitry. Front Mol Neurosci 8, 25.

Mandyam, C.D., Wee, S., Eisch, A.J., Richardson, H.N., Koob, G.F., 2007. Methamphetamine selfadministration and voluntary exercise have opposing effects on medial prefrontal cortex gliogenesis. J. Neurosci. 27, 11442-11450.

Mantilla, C.B., Zhan, W.-Z., Sieck, G.C., 2004. Neurotrophins improve neuromuscular transmission in the adult rat diaphragm. Muscle Nerve 29, 381-386.

Manuel, M., Zytnicki, D., 2011. Alpha, beta and gamma motoneurons: functional diversity in the motor system's final pathway. J. Integr. Neurosci. 10, 243-276. 
Marosi, K., Mattson, M.P., 2014. BDNF mediates adaptive brain and body responses to energetic challenges. Trends Endocrinol. Metab. 25, 89-98.

Martin, K.C., Ephrussi, A., 2009. mRNA localization: gene expression in the spatial dimension. Cell $136,719-730$.

Mayhew, T.M., Momoh, C.K., 1974. Proceedings: A histometric analysis of rat cervical cord: a study of motor horn morphology using the optical microscope. J. Anat. 118, 371.

McDonagh, J.C., Binder, M.D., Reinking, R.M., Stuart, D.G., 1980. Tetrapartite classification of motor units of cat tibialis posterior. J. Neurophysiol. 44, 696-712.

McHanwell, S., Biscoe, T.J., 1981. The localization of motoneurons supplying the hindlimb muscles of the mouse. Philos. Trans. R. Soc. Lond., B, Biol. Sci. 293, 477-508.

Meijer, J.H., Robbers, Y., 2014. Wheel running in the wild. Proc. Biol. Sci. 281.

Meriney, S.D., Dittrich, M., 2013. Organization and function of transmitter release sites at the neuromuscular junction. J. Physiol. (Lond.) 591, 3159-3165.

Milner-Brown, H.S., Stein, R.B., Yemm, R., 1973a. Changes in firing rate of human motor units during linearly changing voluntary contractions. J. Physiol. (Lond.) 230, 371-390.

Milner-Brown, H.S., Stein, R.B., Yemm, R., 1973b. The orderly recruitment of human motor units during voluntary isometric contractions. J. Physiol. (Lond.) 230, 359-370.

Milton, R.L., Behforouz, M.A., 1995. Na channel density in extrajunctional sarcolemma of fast and slow twitch mouse skeletal muscle fibres: functional implications and plasticity after fast motoneuron transplantation on to a slow muscle. J. Muscle Res. Cell. Motil. 16, 430-439.

Milton, R.L., Lupa, M.T., Caldwell, J.H., 1992. Fast and slow twitch skeletal muscle fibres differ in their distribution of $\mathrm{Na}$ channels near the endplate. Neurosci. Lett. 135, 41-44.

Mitchell, T., Barlow, C.E., 2011. Review of the role of exercise in improving quality of life in healthy individuals and in those with chronic diseases. Curr Sports Med Rep 10, 211-216.

Müller, D., Cherukuri, P., Henningfeld, K., Poh, C.H., Wittler, L., Grote, P., Schlüter, O., Schmidt, J., Laborda, J., Bauer, S.R., Brownstone, R.M., Marquardt, T., 2014. Dlk1 promotes a fast motor neuron biophysical signature required for peak force execution. Science 343, 1264-1266.

Munson, J.B., Foehring, R.C., Mendell, L.M., Gordon, T., 1997. Fast-to-slow conversion following chronic low-frequency activation of medial gastrocnemius muscle in cats. II. Motoneuron properties. J. Neurophysiol. 77, 2605-2615.

Muoio, D.M., MacLean, P.S., Lang, D.B., Li, S., Houmard, J.A., Way, J.M., Winegar, D.A., Corton, J.C., Dohm, G.L., Kraus, W.E., 2002. Fatty acid homeostasis and induction of lipid regulatory genes in skeletal muscles of peroxisome proliferator-activated receptor (PPAR) alpha knock-out mice. Evidence for compensatory regulation by PPAR delta. J. Biol. Chem. 277, 26089-26097.

Nader, G.A., Esser, K.A., 2001. Intracellular signaling specificity in skeletal muscle in response to different modes of exercise. J. Appl. Physiol. 90, 1936-1942.

Nakano, H., Masuda, K., Sasaki, S., Katsuta, S., 1997. Oxidative enzyme activity and soma size in motoneurons innervating the rat slow-twitch and fast-twitch muscles after chronic activity. Brain Res. Bull. 43, 149-154.

Nguyen, P.V., Marin, L., Atwood, H.L., 1997. Synaptic physiology and mitochondrial function in crayfish tonic and phasic motor neurons. J. Neurophysiol. 78, 281-294.

Nishimune, H., Stanford, J.A., Mori, Y., 2014. Role of exercise in maintaining the integrity of the neuromuscular junction. Muscle Nerve 49, 315-324. 
Nógrádi, A., Vrbová, G., 2000. Anatomy and Physiology of the Spinal Cord - Madame Curie Bioscience Database - NCBI Bookshelf [WWW Document]. URL http://www.ncbi.nlm.nih.gov/books/NBK6229/ (accessed 6.20.16).

Palay, S.L., 1956. Synapses in the central nervous system. J Biophys Biochem Cytol 2, 193-202.

Pareja-Galeano, H., Sanchis-Gomar, F., García-Giménez, J.L., 2014. Physical exercise and epigenetic modulation: elucidating intricate mechanisms. Sports Med 44, 429-436.

Parikh, H., Nilsson, E., Ling, C., Poulsen, P., Almgren, P., Nittby, H., Eriksson, K.-F., Vaag, A., Groop, L.C., 2008. Molecular correlates for maximal oxygen uptake and type 1 fibers. Am. J. Physiol. Endocrinol. Metab. 294, E1152-1159.

Pedersen, B.K., Febbraio, M.A., 2012. Muscles, exercise and obesity: skeletal muscle as a secretory organ. Nat Rev Endocrinol 8, 457-465.

Pedersen, B.K., Hoffman-Goetz, L., 2000. Exercise and the immune system: regulation, integration, and adaptation. Physiol. Rev. 80, 1055-1081.

Pedersen, B.K., Steensberg, A., Fischer, C., Keller, C., Keller, P., Plomgaard, P., Febbraio, M., Saltin, B., 2003. Searching for the exercise factor: is IL-6 a candidate? J. Muscle Res. Cell. Motil. 24, 113119.

Perry, M.-C., Dufour, C.R., Tam, I.S., B'chir, W., Giguère, V., 2014. Estrogen-related receptor-a coordinates transcriptional programs essential for exercise tolerance and muscle fitness. Mol. Endocrinol. 28, 2060-2071.

Petchey, L.K., Risebro, C.A., Vieira, J.M., Roberts, T., Bryson, J.B., Greensmith, L., Lythgoe, M.F., Riley, P.R., 2014. Loss of Prox1 in striated muscle causes slow to fast skeletal muscle fiber conversion and dilated cardiomyopathy. Proc. Natl. Acad. Sci. U.S.A. 111, 9515-9520.

Peter, J.B., Barnard, R.J., Edgerton, V.R., Gillespie, C.A., Stempel, K.E., 1972. Metabolic profiles of three fiber types of skeletal muscle in guinea pigs and rabbits. Biochemistry 11, 2627-2633.

Pette, D., Heilig, A., Klug, G., Reichmann, H., Seedorf, U., Wiehrer, W., 1985. Alterations in phenotype expression of muscle by chronic nerve stimulation. Adv. Exp. Med. Biol. 182, 169-177.

Pette, D., Staron, R.S., 1990. Cellular and molecular diversities of mammalian skeletal muscle fibers. Rev. Physiol. Biochem. Pharmacol. 116, 1-76.

Petzinger, G.M., Fisher, B.E., McEwen, S., Beeler, J.A., Walsh, J.P., Jakowec, M.W., 2013. Exerciseenhanced neuroplasticity targeting motor and cognitive circuitry in Parkinson's disease. Lancet Neurol $12,716-726$.

Pfeiffer, B.E., Zang, T., Wilkerson, J.R., Taniguchi, M., Maksimova, M.A., Smith, L.N., Cowan, C.W., Huber, K.M., 2010. Fragile $X$ mental retardation protein is required for synapse elimination by the activity-dependent transcription factor MEF2. Neuron 66, 191-197.

Piehl, F., Arvidsson, U., Hökfelt, T., Cullheim, S., 1993. Calcitonin gene-related peptide-like immunoreactivity in motoneuron pools innervating different hind limb muscles in the rat. Exp Brain Res 96, 291-303.

Pontarin, G., Ferraro, P., Bee, L., Reichard, P., Bianchi, V., 2012. Mammalian ribonucleotide reductase subunit p53R2 is required for mitochondrial DNA replication and DNA repair in quiescent cells. Proc. Natl. Acad. Sci. U.S.A. 109, 13302-13307.

Pousinha, P.A., Diogenes, M.J., Ribeiro, J.A., Sebastião, A.M., 2006. Triggering of BDNF facilitatory action on neuromuscular transmission by adenosine A2A receptors. Neurosci. Lett. 404, 143-147.

Pradeepa, M.M., Sutherland, H.G., Ule, J., Grimes, G.R., Bickmore, W.A., 2012. Psip1/Ledgf p52 binds methylated histone $\mathrm{H} 3 \mathrm{~K} 36$ and splicing factors and contributes to the regulation of alternative splicing. PLoS Genet. 8, e1002717. 
Prakash, Y.S., Miller, S.M., Huang, M., Sieck, G.C., 1996. Morphology of diaphragm neuromuscular junctions on different fibre types. J. Neurocytol. 25, 88-100.

Pregelj, P., Trinkaus, M., Zupan, D., Trontelj, J.J., Sketelj, J., 2007. The role of muscle activation pattern and calcineurin in acetylcholinesterase regulation in rat skeletal muscles. J. Neurosci. 27, $1106-1113$.

Prokop, A., Landgraf, M., Rushton, E., Broadie, K., Bate, M., 1996. Presynaptic development at the Drosophila neuromuscular junction: assembly and localization of presynaptic active zones. Neuron 17, 617-626.

Prüfer, K., Muetzel, B., Do, H.-H., Weiss, G., Khaitovich, P., Rahm, E., Pääbo, S., Lachmann, M., Enard, W., 2007. FUNC: a package for detecting significant associations between gene sets and ontological annotations. BMC Bioinformatics 8, 41.

Pun, S., Santos, A.F., Saxena, S., Xu, L., Caroni, P., 2006. Selective vulnerability and pruning of phasic motoneuron axons in motoneuron disease alleviated by CNTF. Nat. Neurosci. 9, 408-419.

Rao, R.R., Long, J.Z., White, J.P., Svensson, K.J., Lou, J., Lokurkar, I., Jedrychowski, M.P., Ruas, J.L., Wrann, C.D., Lo, J.C., Camera, D.M., Lachey, J., Gygi, S., Seehra, J., Hawley, J.A., Spiegelman, B.M., 2014. Meteorin-like is a hormone that regulates immune-adipose interactions to increase beige fat thermogenesis. Cell 157, 1279-1291.

Reichmann, H., Hoppeler, H., Mathieu-Costello, O., von Bergen, F., Pette, D., 1985. Biochemical and ultrastructural changes of skeletal muscle mitochondria after chronic electrical stimulation in rabbits. Pflugers Arch. 404, 1-9.

Reid, B., Slater, C.R., Bewick, G.S., 1999. Synaptic vesicle dynamics in rat fast and slow motor nerve terminals. J. Neurosci. 19, 2511-2521.

Rexed, B., 1952. The cytoarchitectonic organization of the spinal cord in the cat. J. Comp. Neurol. 96, 414-495.

Richter, E.A., Hargreaves, M., 2013. Exercise, GLUT4, and skeletal muscle glucose uptake. Physiol. Rev. 93, 993-1017.

Romanes, G.J., 1951. The motor cell columns of the lumbo-sacral spinal cord of the cat. J. Comp. Neurol. 94, 313-363.

Rose, A.J., Kiens, B., Richter, E.A., 2006. Ca2+-calmodulin-dependent protein kinase expression and signalling in skeletal muscle during exercise. J. Physiol. (Lond.) 574, 889-903.

Safdar, A., Abadi, A., Akhtar, M., Hettinga, B.P., Tarnopolsky, M.A., 2009. miRNA in the regulation of skeletal muscle adaptation to acute endurance exercise in C57BI/6J male mice. PLoS ONE 4, e5610.

Safdar, A., Bourgeois, J.M., Ogborn, D.I., Little, J.P., Hettinga, B.P., Akhtar, M., Thompson, J.E., Melov, S., Mocellin, N.J., Kujoth, G.C., Prolla, T.A., Tarnopolsky, M.A., 2011. Endurance exercise rescues progeroid aging and induces systemic mitochondrial rejuvenation in mtDNA mutator mice. Proc. Natl. Acad. Sci. U.S.A. 108, 4135-4140.

Saha, A.K., Ruderman, N.B., 2003. Malonyl-CoA and AMP-activated protein kinase: an expanding partnership. Mol. Cell. Biochem. 253, 65-70.

Salanova, M., Bortoloso, E., Schiffl, G., Gutsmann, M., Belavy, D.L., Felsenberg, D., Furlan, S., Volpe, P., Blottner, D., 2011. Expression and regulation of Homer in human skeletal muscle during neuromuscular junction adaptation to disuse and exercise. FASEB J. 25, 4312-4325.

Salminen, A., Kauppinen, A., Hiltunen, M., Kaarniranta, K., 2014. Krebs cycle intermediates regulate DNA and histone methylation: epigenetic impact on the aging process. Ageing Res. Rev. 16, 45-65.

Sanchez, A.M.J., Bernardi, H., Py, G., Candau, R.B., 2014. Autophagy is essential to support skeletal muscle plasticity in response to endurance exercise. Am. J. Physiol. Regul. Integr. Comp. Physiol. 307, R956-969. 
Sandrock, A.W., Dryer, S.E., Rosen, K.M., Gozani, S.N., Kramer, R., Theill, L.E., Fischbach, G.D., 1997. Maintenance of acetylcholine receptor number by neuregulins at the neuromuscular junction in vivo. Science 276, 599-603.

Schiaffino, S., Hanzlíková, V., Pierobon, S., 1970. Relations between structure and function in rat skeletal muscle fibers. J. Cell Biol. 47, 107-119.

Schiaffino, S., Mammucari, C., 2011. Regulation of skeletal muscle growth by the IGF1-Akt/PKB pathway: insights from genetic models. Skelet Muscle 1, 4 .

Schiaffino, S., Reggiani, C., 2011. Fiber types in mammalian skeletal muscles. Physiol. Rev. 91, 1447-1531.

Schiaffino, S., Sandri, M., Murgia, M., 2007. Activity-dependent signaling pathways controlling muscle diversity and plasticity. Physiology (Bethesda) 22, 269-278.

Schindelin, J., Arganda-Carreras, I., Frise, E., Kaynig, V., Longair, M., Pietzsch, T., Preibisch, S., Rueden, C., Saalfeld, S., Schmid, B., Tinevez, J.-Y., White, D.J., Hartenstein, V., Eliceiri, K., Tomancak, P., Cardona, A., 2012. Fiji: an open-source platform for biological-image analysis. Nat. Methods 9, 676-682.

Seburn, K., Coicou, C., Gardiner, P., 1994. Effects of altered muscle activation on oxidative enzyme activity in rat alpha-motoneurons. J. Appl. Physiol. 77, 2269-2274.

Sherrington, C.S., 1904. Correlation of Reflexes and the Principle of the Common Path. Nature 70, 460-473.

Sherwin, null, 1998. Voluntary wheel running: a review and novel interpretation. Anim Behav 56, $11-$ 27.

Shimomura, Y., Murakami, T., Nakai, N., Nagasaki, M., Harris, R.A., 2004. Exercise promotes BCAA catabolism: effects of BCAA supplementation on skeletal muscle during exercise. J. Nutr. 134, 1583S$1587 S$.

Smirnova, E., Griparic, L., Shurland, D.L., van der Bliek, A.M., 2001. Dynamin-related protein Drp1 is required for mitochondrial division in mammalian cells. Mol. Biol. Cell 12, 2245-2256.

Song, M.S., Salmena, L., Pandolfi, P.P., 2012. The functions and regulation of the PTEN tumour suppressor. Nat. Rev. Mol. Cell Biol. 13, 283-296.

Spamer, C., Pette, D., 1977. Activity patterns of phosphofructokinase, glyceraldehydephosphate dehydrogenase, lactate dehydrogenase and malate dehydrogenase in microdissected fast and slow fibres from rabbit psoas and soleus muscle. Histochemistry 52, 201-216.

Spielman, L.J., Little, J.P., Klegeris, A., 2014. Inflammation and insulin/IGF-1 resistance as the possible link between obesity and neurodegeneration. J. Neuroimmunol. 273, 8-21.

Stanford, K.I., Goodyear, L.J., 2014. Exercise and type 2 diabetes: molecular mechanisms regulating glucose uptake in skeletal muscle. Adv Physiol Educ 38, 308-314.

Stanford, K.I., Middelbeek, R.J.W., Townsend, K.L., Lee, M.-Y., Takahashi, H., So, K., Hitchcox, K.M., Markan, K.R., Hellbach, K., Hirshman, M.F., Tseng, Y.-H., Goodyear, L.J., 2015. A novel role for subcutaneous adipose tissue in exercise-induced improvements in glucose homeostasis. Diabetes 64, 2002-2014.

Steinbacher, P., Eckl, P., 2015. Impact of oxidative stress on exercising skeletal muscle. Biomolecules 5, 356-377.

Stifani, N., 2014. Motor neurons and the generation of spinal motor neuron diversity. Front Cell Neurosci 8, 293.

Subramanyam, P., Obermair, G.J., Baumgartner, S., Gebhart, M., Striessnig, J., Kaufmann, W.A., Geley, S., Flucher, B.E., 2009. Activity and calcium regulate nuclear targeting of the calcium channel beta4b subunit in nerve and muscle cells. Channels (Austin) 3, 343-355. 
Sugihara, Y., Sadohara, E., Yonezawa, K., Kugo, M., Oshima, K., Matsuda, T., Nadano, D., 2013. Identification and expression of an autosomal paralogue of ribosomal protein S4, X-linked, in mice: potential involvement of testis-specific ribosomal proteins in translation and spermatogenesis. Gene $521,91-99$.

Szabadkai, G., Simoni, A.M., Chami, M., Wieckowski, M.R., Youle, R.J., Rizzuto, R., 2004. Drp-1dependent division of the mitochondrial network blocks intraorganellar $\mathrm{Ca} 2+$ waves and protects against Ca2+-mediated apoptosis. Mol. Cell 16, 59-68.

Takamori, M., 2012. Structure of the neuromuscular junction: function and cooperative mechanisms in the synapse. Ann. N. Y. Acad. Sci. 1274, 14-23.

Talmadge, R.J., Otis, J.S., Rittler, M.R., Garcia, N.D., Spencer, S.R., Lees, S.J., Naya, F.J., 2004. Calcineurin activation influences muscle phenotype in a muscle-specific fashion. BMC Cell Biol. 5, 28.

Tansey, K.E., Botterman, B.R., 1996a. Activation of type-identified motor units during centrally evoked contractions in the cat medial gastrocnemius muscle. I. Motor-unit recruitment. J. Neurophysiol. 75, 26-37.

Tansey, K.E., Botterman, B.R., 1996b. Activation of type-identified motor units during centrally evoked contractions in the cat medial gastrocnemius muscle. II. Motoneuron firing-rate modulation. J. Neurophysiol. 75, 38-50.

Tansey, K.E., Yee, A.K., Botterman, B.R., 1996. Activation of type-identified motor units during centrally evoked contractions in the cat medial gastrocnemius muscle. III. Muscle-unit force modulation. J. Neurophysiol. 75, 51-59.

Thomas, M.G., Loschi, M., Desbats, M.A., Boccaccio, G.L., 2011. RNA granules: the good, the bad and the ugly. Cell. Signal. 23, 324-334.

Tricarico, D., Mele, A., Lundquist, A.L., Desai, R.R., George, A.L., Conte Camerino, D., 2006. Hybrid assemblies of ATP-sensitive $\mathrm{K}+$ channels determine their muscle-type-dependent biophysical and pharmacological properties. Proc. Natl. Acad. Sci. U.S.A. 103, 1118-1123.

Valadi, H., Ekström, K., Bossios, A., Sjöstrand, M., Lee, J.J., Lötvall, J.O., 2007. Exosome-mediated transfer of mRNAs and microRNAs is a novel mechanism of genetic exchange between cells. Nat. Cell Biol. 9, 654-659.

Vanderhorst, V.G., Holstege, G., 1997. Organization of lumbosacral motoneuronal cell groups innervating hindlimb, pelvic floor, and axial muscles in the cat. J. Comp. Neurol. 382, 46-76.

Váraljai, R., Islam, A.B.M.M.K., Beshiri, M.L., Rehman, J., Lopez-Bigas, N., Benevolenskaya, E.V., 2015. Increased mitochondrial function downstream from KDM5A histone demethylase rescues differentiation in pRB-deficient cells. Genes Dev. 29, 1817-1834.

Verstreken, P., Ly, C.V., Venken, K.J.T., Koh, T.-W., Zhou, Y., Bellen, H.J., 2005. Synaptic mitochondria are critical for mobilization of reserve pool vesicles at Drosophila neuromuscular junctions. Neuron 47, 365-378.

Vincent, H.K., Raiser, S.N., Vincent, K.R., 2012. The aging musculoskeletal system and obesityrelated considerations with exercise. Ageing Res. Rev. 11, 361-373.

Vos, M., Lauwers, E., Verstreken, P., 2010. Synaptic mitochondria in synaptic transmission and organization of vesicle pools in health and disease. Front Synaptic Neurosci 2, 139.

Waerhaug, O., Dahl, H.A., Kardel, K., 1992. Different effects of physical training on the morphology of motor nerve terminals in the rat extensor digitorum longus and soleus muscles. Anat. Embryol. 186, $125-128$.

Wang, R.C., Wei, Y., An, Z., Zou, Z., Xiao, G., Bhagat, G., White, M., Reichelt, J., Levine, B., 2012. Akt-mediated regulation of autophagy and tumorigenesis through Beclin 1 phosphorylation. Science 338, 956-959. 
Weiss, N., 2006. The Calcium Channel $\beta 4 a$ Subunit: A Scaffolding Protein Between Voltage-Gated Calcium Channel and Presynaptic Vesicle-Release Machinery? J. Neurosci. 26, 6117-6118.

Westerga, J., Gramsbergen, A., 1992. Structural changes of the soleus and the tibialis anterior motoneuron pool during development in the rat. J. Comp. Neurol. 319, 406-416.

Wigston, D.J., 1990. Repeated in vivo visualization of neuromuscular junctions in adult mouse lateral gastrocnemius. J. Neurosci. 10, 1753-1761.

Wigston, D.J., 1989. Remodeling of neuromuscular junctions in adult mouse soleus. J. Neurosci. 9, 639-647.

Wildner, H., Das Gupta, R., Bröhl, D., Heppenstall, P.A., Zeilhofer, H.U., Birchmeier, C., 2013. Genome-wide expression analysis of Ptf1a- and Ascl1-deficient mice reveals new markers for distinct dorsal horn interneuron populations contributing to nociceptive reflex plasticity. J. Neurosci. 33, 72997307.

Wilson, M.G., Ellison, G.M., Cable, N.T., 2015. Basic science behind the cardiovascular benefits of exercise. Heart 101, 758-765.

Wolpowitz, D., Mason, T.B., Dietrich, P., Mendelsohn, M., Talmage, D.A., Role, L.W., 2000. Cysteinerich domain isoforms of the neuregulin-1 gene are required for maintenance of peripheral synapses. Neuron 25, 79-91.

Wood, S.J., Slater, C.R., 2001. Safety factor at the neuromuscular junction. Prog. Neurobiol. 64, 393429.

Wood, S.J., Slater, C.R., 1997. The contribution of postsynaptic folds to the safety factor for neuromuscular transmission in rat fast- and slow-twitch muscles. J. Physiol. (Lond.) 500 ( Pt 1), 165176.

Woodrow, L., Sheppard, P., Gardiner, P.F., 2013. Transcriptional changes in rat a-motoneurons resulting from increased physical activity. Neuroscience 255, 45-54.

Yamanaka, Y., Honma, S., Honma, K., 2013. Daily exposure to a running wheel entrains circadian rhythms in mice in parallel with development of an increase in spontaneous movement prior to running-wheel access. Am. J. Physiol. Regul. Integr. Comp. Physiol. 305, R1367-1375.

Yamano, K., Fogel, A.I., Wang, C., van der Bliek, A.M., Youle, R.J., 2014. Mitochondrial Rab GAPs govern autophagosome biogenesis during mitophagy. Elife 3, e01612.

Yuan, J.P., Kiselyov, K., Shin, D.M., Chen, J., Shcheynikov, N., Kang, S.H., Dehoff, M.H., Schwarz, M.K., Seeburg, P.H., Muallem, S., Worley, P.F., 2003. Homer binds TRPC family channels and is required for gating of TRPC1 by IP3 receptors. Cell 114, 777-789.

Zajac, F.E., Faden, J.S., 1985. Relationship among recruitment order, axonal conduction velocity, and muscle-unit properties of type-identified motor units in cat plantaris muscle. J. Neurophysiol. 53, 13031322.

Zengel, J.E., Reid, S.A., Sypert, G.W., Munson, J.B., 1985. Membrane electrical properties and prediction of motor-unit type of medial gastrocnemius motoneurons in the cat. J. Neurophysiol. 53, 1323-1344.

Zheng, S., Gray, E.E., Chawla, G., Porse, B.T., O'Dell, T.J., Black, D.L., 2012. PSD-95 is posttranscriptionally repressed during early neural development by PTBP1 and PTBP2. Nat. Neurosci. 15, 381-388, S1.

Zierath, J.R., Wallberg-Henriksson, H., 2015. Looking Ahead Perspective: Where Will the Future of Exercise Biology Take Us? Cell Metab. 22, 25-30.

Zong, H., Ren, J.M., Young, L.H., Pypaert, M., Mu, J., Birnbaum, M.J., Shulman, G.I., 2002. AMP kinase is required for mitochondrial biogenesis in skeletal muscle in response to chronic energy deprivation. Proc. Natl. Acad. Sci. U.S.A. 99, 15983-15987. 


\section{Curriculum vitae}

\section{EDUCATION}

$2015 \mathrm{PhD}$ degree in Neuroscience, from the Göttingen Graduate School for Neurosciences, Biophysics, and Molecular Biosciences, Georg-August University Göttingen, Germany

2011 Master degree in Cellular biology, physiology and pathology, speciality Neurobiology, Paris Descartes University, France

2008 Bachelor degree in Cellular Biology and Physiology, Paris Diderot University, France

\section{PROFESSIONAL EXPERIENCE}

2011-2015 PhD thesis, Georg-August University Göttingen, Germany

European Neuroscience Institute Göttingen

Developmental Neurobiology Lab

2011- 201010 months internship \& 10 months Master thesis

Paris Descartes University, France

Sensori-motricity study centre (CESeM) - CNRS UMR 8194

Team Neuromuscular degeneration and plasticity

2009 internship, Paris Sud University, France

Neurobiology of learning, memory and communication - CNRS UMR 8620

Team Cellular and molecular mechanisms of plasticity and memory

2008 Bachelor thesis, CNRS Gif-sur-Yvette, France

Genetic and integrative neurobiology - CNRS UPR 2216

Team Molecular genetics of circadian rhythms

\section{PUBLICATIONS - POSTER}

Lancelin C, Grosse L, Cherukuri P, Jakobs S, and Marquardt T. (2014). Plasticity-dependent modulation of mitochondrial biogenesis in motor neurons. Poster presentation at the $9^{\text {th }}$ International Motoneuron Meeting, Halifax, NS, Canada.

Biondi O, Branchu J, Ben Salah A, Houdebine L, Bertin L, Chali F, Desseille C, Weill L, Sanchez G, Lancelin C, Aïd S, Lopes P, Pariset C, Lécolle S, Côté J, Holzenberger M, Chanoine C, Massaad C, Charbonnier F. (2015). IGF-1R Reduction Triggers Neuroprotective Signaling Pathways in Spinal Muscular Atrophy Mice. J Neurosci. 35(34):12063-79.

Biondi O*, Branchu J*, Sanchez G, Lancelin C, Deforges S, Lopes P, Pariset C, Lécolle S, Côté J, Chanoine C, and Charbonnier F. (2010). In vivo NMDA receptor activation accelerates motor unit maturation, protects spinal motor neurons, and enhances SMN2 gene expression in severe spinal muscular atrophy mice. J Neurosci. 30(34): 11288-99. 